\title{
Behavioural studies in Prader-Willi Syndrome
}

Citation for published version (APA):

Boer, H. (2004). Behavioural studies in Prader-Willi Syndrome. [Doctoral Thesis, Maastricht University]. Datawyse / Universitaire Pers Maastricht. https://doi.org/10.26481/dis.20041124hb

Document status and date:

Published: 01/01/2004

DOI:

10.26481/dis.20041124hb

Document Version:

Publisher's PDF, also known as Version of record

\section{Please check the document version of this publication:}

- A submitted manuscript is the version of the article upon submission and before peer-review. There can be important differences between the submitted version and the official published version of record.

People interested in the research are advised to contact the author for the final version of the publication, or visit the DOI to the publisher's website.

- The final author version and the galley proof are versions of the publication after peer review.

- The final published version features the final layout of the paper including the volume, issue and page numbers.

Link to publication

\footnotetext{
General rights rights.

- You may freely distribute the URL identifying the publication in the public portal. please follow below link for the End User Agreement:

www.umlib.nl/taverne-license

Take down policy

If you believe that this document breaches copyright please contact us at:

repository@maastrichtuniversity.nl

providing details and we will investigate your claim.
}

Copyright and moral rights for the publications made accessible in the public portal are retained by the authors and/or other copyright owners and it is a condition of accessing publications that users recognise and abide by the legal requirements associated with these

- Users may download and print one copy of any publication from the public portal for the purpose of private study or research.

- You may not further distribute the material or use it for any profit-making activity or commercial gain

If the publication is distributed under the terms of Article $25 \mathrm{fa}$ of the Dutch Copyright Act, indicated by the "Taverne" license above, 
Behavioural studies in Prader-Willi Syndrome 
Layout en druk: Datawyse / Universitaire Pers Maastricht ISBN $90-5278-441-8$ 


\title{
Behavioural studies in Prader-Willi Syndrome
}

\author{
Proefschrift
}

ter verkrijging van de graad van doctor aan de Universiteit Maastricht, op gezag van de Rector Magnificus, Prof. mr. G.P.M.F. Mols, volgens het besluit van het College van Decanen, in het openbaar te verdedigen, op woensdag 24 november 2004 om 14.00 uur.

\author{
door \\ Gezinus Harm Boer \\ geboren op 22 Augustus 1959 te Delft
}

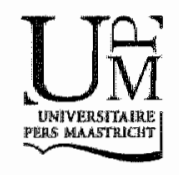


Promotores:

Prof. dr. L.M.G. Curfs

Prof. dr. J.P.M. Geraedts

Beoordelingscommissie:

Prof. dr.J.J. van Os (voorzitter)

Prof. dr. J.P. Fryns (University Leuven, Belgium)

Prof. dr. G.G.K. Gillessen-Kaesbach (University Essen, Germany)

Prof. dr. H.L.G.J. Merckelbach

Prof. dr. G. O'Brien (University of Northumbria, United Kingdom)

Some of the studies described in this study were funded by the Wellcome Trust, with additional financial support by the Prader-Willi Syndrome Association UK. 


\section{Paranimifen Jaap Neerken Jannet van der Spek}

Voor Racleel 



\section{Contents}

1 The Study 9

1.1 Introduction 10

1.2 Clinical fearures 10

1.3 Prevalence 16

1.4 Genetics 17

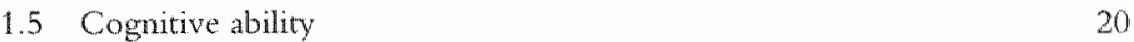

1.6 Maladaptive behaviour 22

1.7 Compulsive and ritualistic behaviours 25

1.8 Prader-Willi syndrome and psychosis 27

1.9 Behaviour and event related brain activity 28

2 Population prevalence and estimated birth incidence and mortality rate for people with Prader-Willi Syndrome in one UK Health Region

3 A Cognitive abilities and genotype in a population-based sample of people with Prader-Willi syndrome

3B Academic underachievement by people with Prader-Willi syndrome

4A Problem Behaviors Associated with Deletion Prader-Willi, Smith-Magenis, and Cri Du Chat Syndromes

4B Development and behaviour in genetic syndromes:

Prader-Willi Syndrome

5 Prader-Willi syndrome, compulsive and ritualistic behaviours:

The first population-based survey

6A Prader-Willi syndrome and psychotic symptoms:

Case descriptions and genetic studies

6B Psychotic illness in people with Prader-Willi syndrome due to chromosome 15 maternal uniparental disomy

7A Differences in behavioural phenotype between parental deletion and maternal uniparental disomy in Prader-Willi syndrome 
7B Prader-Willi syndrome: Behavioural differences between deletion and disony subtypes

8 Discussion $\quad 177$

8.1 General Comments 178

$\begin{array}{ll}8.2 & \text { Population Prevalence } \\ 8.3 & 178\end{array}$

$\begin{array}{ll}8.3 \text { Cognitive functioning } & 179\end{array}$

8.4 Maladaptive behaviour 180

8.5 Obsessive-compulsive symptoms $\quad 1.82$

8.6 Prader- Willi syndrome and psychotic disorders 183

8.7 Prader-Willi Syndrome and event related brain activity 186

8.8 Concluding comments and future research 186

$\begin{array}{lr}\text { Sumary } & 191\end{array}$

$\begin{array}{lr}\text { Samenvatting } & 195\end{array}$

$\begin{array}{lr}\text { Dankwoord } & 199\end{array}$

$\begin{array}{lr}\text { Curriculum Vitae } & 203\end{array}$

$\begin{array}{ll}\text { Publications } & 204\end{array}$

$\begin{array}{ll}\text { Appendix } 1 & 207\end{array}$

Maladaptive Behaviour in Prader-Willi Syndrome in adult life

Appendix 2

Development and behaviour in genetic syndromes:

Rubinstein-Taybi Syndrome. 
Chapter 1

The Study 


\subsection{Introduction}

By the early 1990's (postal) questionnaire-based studies (Whitman \& Accardo, 1987; Greenswag, 1987; Clarke et al. 1989) had suggested thata number of behaviours may be typical of Prader-Will syndrome (PWS), but no studies using controls had yet been published. We therefore set out to discover whether the behaviouts that had been found were typical of PWS or whether they could be explained through other means, such as the fact that most people with PWS have mental retardation (learning disability), and many are obese. A number of articles (e.g. Whitman and Accardo) had suggested that obsessive-compulsive symptoms, and psychosis were more commonly found in PWS, and we set out to test this.

In a population study we attempted to establish birth incidence, prevalence and mortality rates, and we looked at cognitive functioning of people with PWS. We compared maladaptive behaviours in PWS with those occurring in a number of other disorders caused by chromosomall deletions. Compulsive behaviours were investigated in the population-based study, and we examined whether psychotic symptoms were more common in those with PWS, both in an international case report study, and as part of the population study. In order to confirm the differences in cognitive abilities between PWS caused by deletion, and PWS caused by disomy, we compared the two subgroups using neuro-physiological studies.

\subsection{Clinicall features}

Prader...Willi syndrome was first described by Prader ef al. who in 1956 publisheda report in the Schweizerische Medizinishe Wochenschrift with the title "Ein Syndrom von Adipositas, Kleinwuchs, Kryptorchismus und Oligophrenie nach myatonieartigem Zustand in Neugeborenalter" (A syndrome of obesity, short stature, hypogonadism and mental retardation, with hypotonia during the neonatal period). This article had in 1995 not yet been translated into English, and we translated this article, together with an overview of the literature (Clarlee $D$., Boet H. \& Webl T. 1995). The original article by Prader et al. concentrated on the medical hindings of children and adolescents with PWS. They described many of the important features of PWS, and the Consensus Diagnostic Criteria (Holm et al. 1993) of the disorder were largely based on features described in the original report. Pradere al described the extreme neonatal hypotonia, with lack of movement and inability to cry or suck, followed by obesity (due to an abnomal appetite) around the age of two years. Around this age growth retardation and mental retardation became apparent. Acromicria (small hands and feet) and other dysmorphic features, and (in males) a hypoplastic, flat, scotum with inguinal or abdominal retention of the testes were characteristic. They also described a young man who became diabetic at the age of 17 years. When further articles were 
published, and with clinical experience, it became apparent that behavioural characteristics such as outbursts of temper, obsessional traits and stubbornness, as well as clinical features such as central adiposity, sleep disorders, abnormalities of temperature and pain perception were common (Whittington et al. JMG 2002). which resulted in the Consensus Diagnostic Criteria.

PWS is associated with hypotonia and difficulty in feeding in infancy, followed by overeating, usually starting between the ages of one to four years. People with PWS have a characteristic facial appearance with almond-shaped eyes and down-turned comers of the mouth. Most people with PWS have a mild learning disability. The intense preoccupation with food is one of the most striking features of the syndrome, and can lead, if not controlled, to extreme obesity. Food is often locked away by carers, and people with PWS have been reported to eat unsuitable, such as frozen or rotten, food. The disturbed balance of food intake and energy metabolism is probably caused by a diminished satiety response (Holland et al. 1993). The management of the eating disorder in PWS causes difficult ethical dilemmas (Holland and Wong, 1999; Van Hooren et al. 2002). Holland and Wong commented that it may be possible to control access to food with the full consent of the person with PWS, but argued that allowing serious weight gain in the absence of careful considerations of issues such as capacity, the person's best interests, and the least restrictive alternative, would be an abdication of responsibility. Excessive daytime sleepiness is common, and sleep-apnoea and disturbances in REM-sleep have been described (Laurance et al. 1981; Vela-Bueno et al. 1984; Clarke et al. 1989; Helbing-Zwanenburg et al. 1993; Richdale et al 1999). Vela-Bueno et al. suggested that sleep abnormalities in PWS could be caused by hypothalamic dysfunction. Harris and Allen (1996) found that sleep-disordered breathing was related to the degree of obesity in patients with PWS and that these were diminished by weight loss. However, excessive daytime sleepiness persisted, despite weight changes. Richdale et al. (1999) found that, compared to a non-learning disabled control group, children and adults with PWS were much more likely to have excessive daytime sleepiness. Cardiac failure and respiratory problems are amongst the most common cause of death (Laurance et al. 1981; Butler et al. 2002, Schrander-Stumpel et al. 2004; Vogels ef al. 2004), although Stevenson et al. (2004) found that adrenal weight was small in a number of people with PWS who had died, and suggested further research into adrenal insufficiency in PWS. People with PWS may have a reasonable life expectancy if obesity can be avoided (Carpenter, 1994).

Holm et al. (1993) proposed the Consensus Diagnostic Criteria for PWS which were developed by consensus of seven clinicians experienced with the syndrome in consultation with national and international experts. A weighted score of eight or more (if aged over three years) and five or more (if aged under four years), based on the presence of eight major (scored as one) and eleven minor (scored as half) 
was needed for a diagnosis of PWS to be made (see table 1). There was no requirement that any particular criterion is present, as long as the total number exceeded the given limit. At the time of the Consensus Diagnostic Criteria, PWS was still essentially a clinical diagnosis, and the criteria therefore have to be interpreted with caution as, if Prader-Will syndrome is defined in terms of physical or behawioural features, a large proportion of affected people will have such features.

We attempted to identify all people with $\mathrm{PWS}$ in a region of the UK comprising of approximately 5 million people (Whittington ef al. 2002). Ninety-three out of a total of 124 nominations of possible cases agreed to take part. Sixty-one of this group met genetic criteria for PWS and 19 had negative genetic findings. The other 13 did not give blood samples and had no previous genetic records. Other patients with PWS from outside the region were recruited to increase numbers (particularly of those with disomy). Of these, 42 had positive genetic tests. In addition, a comparison group of 22 people with learning disabilities of other aetiologies was recruited to add to the ten people from the original group of 93 who did. not meet either clinical or genetic criteria. Genetic diagnoses were made by either obtaining blood samples or by examining genetic records. We then analysed the frequencies of clinical diagnostic symptoms and characteristic PWS behaviours for those with a genetic diagnosis with a comparison group that was negative both clinically and genetically. Table 2 gives the derivation of these gromps.

Table 1 compares the Consensus Diagnostic Criteria with our findings (Whittington at al 2002). We reported that if genetic testing was positive (i.e. whenever genetic testing indicated abnomal methylation at SNRPN; see chapter 1.4) some clinical features were always present including floppy at birth, weak cry or inactivity, poor suck, feeding difficulties and hypogonadism. We concluded that these five "core symptoms" together with an eating disturbance and learning disability constitute the "all or none" characteristics of PWS. A definite "no" to any of these questions always coincided with genetic testing being negative (i.e. the finding of a nomal methylation pattern; no-one who answered no to any of the core symptoms had a genetic diagnosis of PWS). However, seven people out of our two comparison groups with a total of 51 patients with nomal methylation patterns fulfilled the first four criteria, and three of these fulfilled all five (i.e. some people who fulfilled the first four or even five core criteria did not have PWS). In addition, Gunay-Aygun er al. (2001) tound that, of patients with positive genetic testing for PWS, over $15 \%$ did not meet the Consensus Diagnostic Criteria retrospectively (i.e. some people who had genetic diagnosis of PWS did not meet the criteria for a clinical diagnosis of PWS). In recent years, PWS has more and more been regarded as a genetic syndrome, and the purpose of clinical diagnostic criteria has shifted from making a diagnosis to raising suspicion of a diagnosis of PWS. Gunay-Aygun et al. (2001) argue that the threshold for testing of PWS should be lowered, as knowledge of the diagnosis can have a significant effect on outcome. 
Table 1. Comparison of Consensus Diagnostic Criteria (Holm et al. 1993) with the findings based on the 103. people with PWS in the population-based study (Whittington et al. 2002)

Consensus Diagnostic Criteria: Holm et al.

Findings of Whittington et al.

\section{Major criteria}

Neonatal and infantile central hypotonia with poor sucke, gradually improving with age

Feeding problems in infancy with need for special feeding techniques and poor weight gain/failure to thrive

Excessive or rapid weight gain on weight for length chart (excessive defined as crossing 2 centile channels) after 12 months but before 6 years of age; central obesity in the absence of intervention

Characteristic facial features with dolichocephaly in infancy, narrow face or bifrontal diameter, almond shaped eyes, small appearing mouth with thin upper lip, down-turned corners of the mouth ( 3 or more required)

Hypogonadism with any of the following, depending on age:

(A) Genital hypoplasia, male scrotal hypoplasia, cryptorchism, small penis and/or testes for age ( $<5$ th centile); female: absence or severe hypoplasia of labia minora and/or clitoris

(B) Delayed or incomplete gonadal maturation, with delayed pubertal signs in the absence of intervention after 16 years of age (male: small gonads, decreased facial and body hair, lack of voice change; female:

amenorrhoea/oligomenorrhoea after age 16)

Global developmental delay in a child younger than 6 years of age; mild to moderate mental retardation or learning problems in older children

Hyperphagia/food foraging/obsession with food

Deletion $15 q 11-13$ on high resolution ( $>650$ bands) or other cytagenetic/molecular abnormality af the PWS chromosome region, including maternal disomy
Severe floppiness at birth with later

improvement

Poor suck at birth with later improvement

Difficulty feeding at birth (need for special feeding techmiques)

obesity during childhood, age when noted, maximum weight ever and age when reached
Males: undescended testes, surgery on testes decreased facial and body hair, lack of voice change, early pubertal signs, small penis

Females: does/diid she have periods (no if only when on pill), age of first period, how many periods per year, early pubertal signs

Learning difficulties

Tendency to overeat/obsession with food 
Consensus Diagnostic Criteria: Holm et al.

\section{Minnor criteria}

Decreased foetal movement or infantile lethargy or weak cry in infancy, improving with age

Characteristic behaviour problems: temper tantrums, violent outbursts and obsessive-compulsive behavior, tendency to be argumentative, oppositional, rigid, manipulative, possessive, and stubbom: perseverating, stealing and lying (5 or more of these symptoms required.

\section{Sleep disturbance or sleep apnoea}

Short stature for genetic background by age 15 (in the absence of growth hormone intervention)

Hypopigmentation: fair skin and hair compared to family

Small hands ( $<25^{\text {th }}$ centile) and/or feet $\left(<10^{\text {th }}\right.$ centile) for height age

Narrow hands with straight ulnar bonder

Eye abnormalities (esotropia, myopia)

Thick viscous saliva with crusting of corners of mouth

Speech articulation defects

Skin picking

Supportive findings

High pain threshold

Decreased vomiting

Temperature instability in infancy or altered temperature sensitivity in older children and andults

Scoliosis and/or kyphosis

Early adrenarche

osteoporosis, thin bones, eg easily broken

Unusual skill with jigsaw puzzles

Normal neuromuscular studies
Findings of Whittington et al.

Decreased movement of baby during pregnancy

Weak cry or inactivity as a baby

Frequency and severity of any: temper tantrums, skin picking, repetitive questioning. obsessional behaviour, violent/aggressive behaviour, fluctuations in mood. argumentative, lying, stealing stubborn, any other behaviour problems

Disturbed or noisy sleep

Short height: heights of proband, mother, father, height centile. Growth hormone ever (dates)

Fair skin and hair compared with family

Small hands or feet (parents \& researchers agree)

Eye problems (short sight, long sight, squint)

Thick saliva with crusting of corners of mouth

Difficulty with anticulating words

Wigh pain threshold (examples)

Ever vomited: circumintances, frequency $(0$. <normal, normal)

Not feeling hot or cold when others are: abnormal temperature response

Scoliosis or spinal curvature

Unusual skill with jigsaw puzzles

From: Whittington J. E., Holland A. J., Webb T., Butler J. V., Clarke D. J. \& Boer H. (2002) Relationship between clinical and genetic diagnosis of Prader-Willi syndrome. Joumal of Medical Genetics 39, 926-32. 
Table 2. Analysis of the frequencies of clinical diagnostic symptoms and characteristic PWS behaviours for those with a genetic diagnosis, contrasted with a comparison group that was negative for both clinical and genetic criteria.

\begin{tabular}{|c|c|c|c|c|c|c|}
\hline \multirow[b]{2}{*}{ Criterion } & \multicolumn{3}{|c|}{$\begin{array}{l}\text { Genetics positive } \\
n=103\end{array}$} & \multicolumn{3}{|c|}{$\begin{array}{l}\text { Genetics negative } \\
n=32\end{array}$} \\
\hline & res & Mild & No & Yes & Mild & No \\
\hline Severe floppiniess & 96 & 5 & 0 & 4 & 4 & 22 \\
\hline Poor suck & 94. & 5 & 0 & 4 & 1 & 25 \\
\hline Difficulty breathing & 98 & 2 & 0 & 11 & 1 & 25 \\
\hline Decreased movement & 65 & 7 & 23 & 2 & 3 & 20 \\
\hline Weak cry/inactive & 94 & 6 & 0 & 2 & 4 & 22 \\
\hline Childhood obesity & 77 & 4 & 18 & 17 & 2 & 12 \\
\hline Overeat/food obsess & 78 & 12 & $\mathbb{1 1}$ & 18 & 3 & 10 \\
\hline Hypogonadism & 54 & $a$ & 0 & 4 & 1 & 9 \\
\hline Periods per year & 22 & 8 & 0 & 1 & 0 & 7 \\
\hline Disturbed/noisy sleep & 56 & 10 & 34 & 13 & 2 & 16 \\
\hline Short height & 73 & 1 & 23 & 10 & 0 & 21 \\
\hline Fair for family & 7 & 49 & 43 & 2 & 11 & 18 \\
\hline Small hands or feet & 85 & 11 & 3 & 6 & 7 & 16 \\
\hline Eye problems & 74 & 1 & 24 & 17 & 0 & 14 \\
\hline Thick saliva & 55 & 23 & 21 & 17 & 0 & 27 \\
\hline Articulation problems & 65 & 16 & 16 & 17 & 4 & 10 \\
\hline High pain threshold & 50 & 29 & 10 & 3 & 13 & 11 \\
\hline Temperature intensity & 44 & 11 & 30 & 3 & 1 & 22 \\
\hline Skill with jigsaws & 28 & 14 & 48 & 0 & 3 & 28 \\
\hline Stubborn & 80 & 14 & 3 & 18 & 5 & 9 \\
\hline Scoliosis & 30 & 8 & 2 & 0 & 4 & 28 \\
\hline Reduced vomiting & 46 & 39 & 10 & 4 & 7 & 19 \\
\hline Severity eating & 45 & 24 & 27 & 3 & 10 & 18 \\
\hline Possessive & 47 & 31 & 24 & 11 & 8 & 12 \\
\hline Skin picking & 56 & 19 & 25 & 3 & 9 & 20 \\
\hline Temper tantrums & 63 & 26 & 4 & 15 & 9 & 8 \\
\hline Repeated questions & 63 & 12 & 20 & 7 & 5 & 18 \\
\hline Obsessional behaviour & 66 & 26 & 6 & 8 & 14 & 10 \\
\hline Violent behaviour & 34 & 33 & 28 & 11 & 6 & 15 \\
\hline Mood swings & 34 & 18 & 48 & 6 & 7 & 19 \\
\hline Argumentative & 37 & 28 & 27 & 12 & 5 & 13 \\
\hline Lying & 34 & 25 & 32 & 9 & 7 & 12 \\
\hline Stealing & 40 & 23 & 30 & 6 & 4 & 21 \\
\hline
\end{tabular}

From: Whittington J.E., Holland A.J., Webb T., Butter J.W., Clarke D.J. \& Boer H. (2002) Relationship between clinical and genetic diagnosis of Prader-Willi syndrome. Jounal of Medical Genetics 39, 926-32. 


\subsection{Prevalence}

Zellweger and Soper (1979), based on the number of referrals to their service, estimated that PWS occurs in about 1 in 25000 live births, and Butler (1990) commented that PWS is the most common syndromal cause of marked obesity. Few population studies have been reported on PWS. Burd et al. (1990) surveyed paediatricians, neurologists, and clinical geneticists in North Dakota, and contacted other institutions including a group home for people with $\mathrm{PWS}$. They had a $99 \%$ response rate and they found 17 people with $\mathrm{PWS}$ with ages between 9 and 30 years. The authors concluded that this implied a prevalence rate of about 1 in 16000 for that age range or about 1 in 38000 for the entire population. Burd et al. felt that the true population prevalence may have been greater than 1 in 16000 as some doctors may not have been contacted and some people may not have been recognised by the description which the authors provided. The population of North Dakota for the age range of between 9 and 30 at the time was about 263000 . The authors did not indicate how many genetic diagnoses were included.

Akefeldt ef al. (1991) estimated the prevalence of PWS in the age range of 0 to 35 years in the county of Skaraborg, a rural part of Sweden with a total population of about 273000 , by writing to doctors and other and other professionals working in different fields of medicine, rehabilitation and mental disabilities. All people with possible PWS were examined by a newropediatrician, a child psychiatrist, a child psychologist, and a speech pathologist. Clinical diagnoses were made. Eleven people were diagnosed as definitely having PWS and four more were thought to have a probable diagnosis of PWS. This resulted in an estimate of a population prevalence of "clear PWS" in the ages between 0 and 25 years of 1 in 8500 , and if those with probable PWS were included, of between 1 in 6700 and 1 in 5000 .

We attempted to ascertain all people with PWS in the old Anglia and Oxfond regions in England (population five million). This study is described in chapter 3 (Whitington J.E., Holland A.J., Webb T., Butler J.V., Clarke D.J. \& Boer H. (2001) Population prevalence and estimated birth incidence and mortality rate for people with Prader-Willi Syndrome in one UK Health Region. Joumal of Medical Gentro 38, 792-8). Our aim was to describe the population prevalence by locating all definte or possible cases of PWS in this region of the UK, including all ages, and using, as far as possible, confimed genetic diagnoses. 


\subsection{Genetics}

\subsubsection{Introduction}

PWS results from abnormal or absent expression of imprinted, patemally expressed gene or genes on the long arm of chromosome 15 at the genetic locus 15q11-13. These genes have not yet been identified. The two main genetic sub-types are a deletion at the locus 15q11-13 involving chromosome 15 of paternal origin (approximately $70 \%$ of cases) (Ledbetter $e t$ al. 1981) and maternal uniparental disomy of chromosome 15 (approx $25 \%$ of cases) (Nicholls et al. 1989). The remaining 1\%-5\% have a chromosomal re-arrangement or a chromosome 15 imprinting defect (Buiting et al. 2003).

\subsubsection{Prader-Willi syndrome and Angelman Syndrome}

Although both deletions and uniparental disomy affecting chromosome 15 are seen in PWS and in Angelman syndrome (AS), the two disorders are phenotypically very different. AS is characterised by severe mental retardation, severe speech problems or absent speech, jerky movements, ataxia, tongue protrusion, episodes of inappropriate laughter and a "happy disposition", and a characteristic electro-encephalogram. (Angelman, 1965; Boyd ef al. 1988). Hypopigmentation is common in both disorders and so are cognitive impairments (although the latter are usually mild in PWS and severe in AS) (Pembrey at al. 1989; Cassidy and Schwartz, 1998).

Both AS and PWS are characterised by genomic inmprinting (Cassidy and Schwartz, 1998; Buiting ef al. 2003). The genes for the two disorders are located very close together, and these disorders may result from the same chromosomal deletion. The parent of origin of the chromosome 15 that becomes deleted determines which disorder results. PWS and AS can also be the result of having both chromosomes 15 derived from one parent, known as uniparental disomy. Both PWS and its sister syndrome AS can therefore result from an apparently similar loss of material in $15 \mathrm{q} 11 \mathrm{q} 13$ (Pembrey et al. 1989). They can also result from a structural abnormality of the imprinting centre, which is known as an imprinting mutation or imprinting defect (Buiting, 1995).

In about $20 \%$ of people with AS, the syndrome has been associated with mutations in a single gene, the E6AP ubiquitin ligase gene UBE3A, although other genes may also be implicated. This gene is imprinted in the brain (Kishino ef al. 1997; Matsuura et al. 1997). 


\subsubsection{Prader-Willi syndrome and chromosome 15 abnormalities}

In 1981 Ledbetter al. found small deletions with breakpoints in bands $15 q 11$ and 15 q13 in four out of five patients with PWS. In their fist case one chromosome was shown to be slightly shorter in routine mid-metaphase chromosome analysis than its homologue, with an apparent deletion in the proximal (nearest to the centrey area of the Jong arm of Chromosome 15 . Using prophase analysis with G-banding they confirmed a deletion with breakpoints in bands $q 11$ and $q 13$, which led to a total loss of band $q 12$ and partial loss of bands $q 11$ and $q 13$. Three of the four other patients had nearly identical deletions, but the fifth case had an apparently normal karyotype. The authors concluded that an appreciable number of cases of PWS may be caused by deletion of the proximal portion of the longarm of chromosome 15.

The introduction of molecular techniques (studying the DNA, rather than the physical structure and appearance of chromosomes) meant that smaller deletions were found in both PWS and AS (Donlon, 1988). The $15 \mathrm{q}$ deletion arises as anew deletion ("de novo") in both syndromes, but in PWS it always affects the chromosome 15 inherited from the father, while in AS the deleted chromosome is inherited from the mother (Williams et al. 1990; Cassidy and Schwartz, 1998). The parental origin of number 15 chromosomes can be detemined cytogenetically (through inherited polymorphisms, or visible differences, of the centromeric region) and by molecularstudies using restriction fragment length polymorphisms (RFLPS) in the $15 \mathrm{q} 11 \mathrm{q} 13$ region.

Nicholls et al. (1989) showed that maternal uniparental disomy (UPD) could cause PWS. They reported a man with PWS who had inherited an apparently balanced $13 \mathrm{q} 15 \mathrm{q}$ Robertsonian translocation from his mother. His half sister had inherited the same chromosome, but did not have PWS. Using probes from $15 q 11 \mathrm{q} 13$, Nicholls et al. demonstrated that the affected man had inherited the maternal chromosome 15 involved in the translocation, and had also inherited another chromosome 15 from his mother, but no chnomosome 15 from his father. By 1993 Nicholls reported that UPD could account for $25 \%$ of al] PWS patients. Nicholls et a. (1989) suggested that the clinical proband seemed to arise from the absence of a paternal contribution to region $15 \mathrm{q} 1 \mathrm{lq} / 3$ rather than from a specific gene mutation, and that this implied functional differences in alleles of a gene or genes from this region of the genome that depend on the sex of the transmitting parent. This is known as genetic imprinting. The authors concluded that the association of PWS with maternal UPD for the region $15 \mathrm{q}^{1} 1 \mathrm{q} 13$ implicated a role for imprinting in the atiology of PWS. UPD involves the entire chromosome 15 , but only the $15 q 11 \mathrm{q} 13$ region is imprinted. Imprinting can be seen as "parent of offspring differences" in the expression of genetic traits (Dimitropoulos et al. 2000). In a region on the chromosome where imprinting occurs, only one copy of the genes is 
transcribed. In PWS the genes derived from the mother are inactivated (imprinted) in a process known as methylation (adding a methyl group to cytosine bases in the DNA chain).

In a small number of patients PWS is caused by an imprinting defect, where the imprinting centre fails to reset the paternal imprint, which leads to an apparent disomy, although the contribution from the other parent is actually present (i.e. both the maternal and paternal chromosome 15 are present) (Buiting et al. 1995; Buiting et al. 2003).

PWS and AS have different critical regions on chromosome 15. Kuwano et al. (1992) reported that evidence from yeast artificial chromosome (YAC) cloning (to produce multiple copies of DNA fragments) suggests that the critical regions are contiguous (next to each other), with the PWS criticall region (PWSCR) lying proximal to that for AS (ASCR). The PWSCR and ASCR are oppositely imprinted. Most of the deletions that have been detected in PWS and AS include both critical regions and have similar breakpoints. The expression of imprinted genes (the size of the "imprinted domain" is about $2 \mathrm{Mb}$ ) is controlled by an imprinting centre, a genetic element functional in early development that regulates differences in replication timing, DNA methylation, and chromatin structure (Nicholls and Krepper, 2001).

The $15 \mathrm{q} 11 \mathrm{q} 13$ region is still poorly defined as few patemally expressed genes have so far been identified, and, to date, no single mutation in any of them has definitely been associated with any clinical signs or symptoms (Webbet al. 2002). Özçelik et al. (1992) mapped the gene encoding small nuclear ribonucleoprotein associated polypeptide SmN SNRPN to PWSCR. The SNRPN gene is imprinted, and only the paternally derived allele is expressed in the brain. However, Özçelik ef al. commented that the PWSCR region into which. SNRPN falls is likely to contain other maternally imprinted genes, and that other genes may contribute to the PWS phenotype. SNR PN was thought to be a strong candidate gene, but PWS is thought to be a multigenic disorder, and the result of the non-functioning of several genes.

Most patients with PWS (about 70\%) have a deletion of about $4 \mathrm{Mb}$. In these patients all of the non-imprinted genes are therefore only present as a single copy (haploid insufficiency). It is therefore possible that haploid insufficiency could contribute to the phenotype in those with a deletion, but not in those with maternal disomy. Maternal disomy would, in contrast, result in a double dose of any imprinted genes that are nomally silenced in the paternal homolog of chromosome 15, but which remain active in the maternal homolog (Table 3) (Webbet al. 2002). Non-imprinted genes present in either the proximal part of $15 \mathrm{q} 11 \mathrm{q} 13$ (PWSCR) or the distal part (ASCR) are present in two copies in both nomal individuals and those with disomy, but in only a single copy in those with a deletion. People with normal chromosomes 15 and patients with PWS with deletions will 
Table 3. Possible differences between deletion and disomy probands with PWS

\begin{tabular}{|c|c|c|c|}
\hline Mutation & $\begin{array}{l}\text { Maternally } \\
\text { imprinted genes }\end{array}$ & Non-iniprinted genes & $\begin{array}{l}\text { Paternally imprinted } \\
\text { genes }\end{array}$ \\
\hline$\approx 4 M b$ deletion & Deficiency & $\begin{array}{l}\text { Possible } \\
\text { haptornsufficiency }\end{array}$ & No effect \\
\hline Maternal UPD & Deficiency & No effect & Twofold excess \\
\hline Imprinting centre mutation & Deficiency & No effect & Twofold excess \\
\hline
\end{tabular}

From: Webb T., Whittington J., Clarke D., Boer H., Butler J. \& Holland A. (2002) A study of the influence of different genotypes on the physical and behavioral phenotypes of children and adults ascertained clinically as having PWS. Chinical genetics 62, 273-81.

have single copies of maternally imprinted genes located within the distal (ASCR) part of $15 \mathrm{q} 11 \mathrm{q} 3$, but those with maternal UPD have two active copies. An excess of genetic material has a less severe effect than a loss, and a deletion would usually be expected to be more severe than UPD, but it is unusual that the presence of such a large excess expression of chromosomal material has apparently such a small effect. A relatively small effect can be explained if a mechanism similar to $\mathrm{X}$-inactivation exists (Webb et al, 2002).

Gallagher ef al. (2002) proposed that the minimal critical region for PWS was about $121 \mathrm{~kb}$ within the SNRPN locus at $15 \mathrm{q} 11 \mathrm{q} 13$. They suggest that this region contains the PWCR1/HBII-85 snoRNA cluster and the HBII-38A snoRNA as the only putative functional genes. It has not yet been demonstrated whether these genes are functional.

Butler et al. (2004) proposed that the deletion subgroup of PWS can be subdivided in two further classes, as the breakpoints appear to occur in a relatively small region of chromosome 15, type I and type II. The breakpoint of type I is close to the centromere, and that of type $\mathrm{ll}$ is about $500 \mathrm{~kb}$ more distal. The deletion of type $\mathrm{I}$ is therefore about $500 \mathrm{~kb}$ larger. Butler er al (2004) found that a number of psychological, behavioural, and academic achievement scores were significantly worse in people with PWS and type I deletions, compared to those with UPD or type II deletions, and that people with type II deletion also needed closer levels of supervision. Those with longer deletions were more likely to have obsessive-compulsive symptoms and problems with vistal perception, but the levels of self-injury were not significantly different over the three groups.

\subsection{Cognitive ability}

Patients with PWS have a wide range in intellectual functioning. The intelligence of some of the patients is in the borderline and nomal range. Curfs et al. (1991) 
noted that people with PWS have high scores on the block design subtest of the WISC-R, reflecting skill in recognizing and evaluating figure relations. Analysis of subtest scores and a comparison of differences between verbal and non-verbal IQ found that individuals with PW/S had better vistual motor discrimination skills than skills in the processing of auditory (verbal) information. This suggests that children and young adults with PWS may be relatively skilled at visual information processing, and unskilled at processing verbally presented information. This can explain why people with PWS are sometimes said to ignore or misinterpret spoken information, but are able to comprehend the same information if it is presented graphically.

Curfs \& Fryns (1991) summarised data from 57 studies including 575 people with PWS, and concluded that about $5 \%$ had overall cognitive abilities clearly above the range associated with mental retardation (IQ>85), $28 \%$ had borderline cognitive abilities. (IQs between 70 and 85 ), 34\% had mild retardation, $27 \%$ moderate, $5 \%$ severe, and less than $1 \%$ profound mental retardation. There did not appear to be any association between global cognitive ability and sex., and no differences in the distribution of intelligence scores in adults compared with children.

It is now generally accepted that, as a group, people with. PWS have better visuospatial skills, have poor short-term memories and Dykens at al (1992) reported that they have exceptional skills in jigsaw puzzles, and that the $1 Q$ is not related to weight or age.

In recent years comparisons have been made of cognitive performance between the two main (deletion and UPD) genetic subgroups of PWS. Roof et al. (2000) found that those with UPD had significantly higher verbal intelligence scores than those with delecion $(p<0.01)$, but that there was no difference in the average performance (or non-verbal) intelligence. They found that people with disomies scored higher on most verbal subscale mean scores, but that the mean object assembly subscale score was higher for people with deletions.

In our study on cognition (chapter 3a: Whittington J., Holland A., Webb T. Butler J., Clarke D. \& Boer H. (2004) Cognitive abilities and genotype in a population-based sample of people with Prader-Willi syndrome. Joumal of Intellectwal Disability Research 48, 172-87) the aim was to establish what mechanisms can explain the distribution of cognitive ability in those with PWS and to find whether there were any systematic cognitive differences between the deletion and UPD subgroups of PWS. We investigated whether there are any differences between the subgroups, what these may say about the genetic influences on cognition, and what implications this may have for the understanding of cognitive development in the general population.

In chapter 36 (Whittington J., Holland A., Webb T., ButlerJ., Clarke D. \& Boer H. (2004) Academic underachevement by people with Prader-Willi syndrome. Joumal of Intellectual Disability Research 48, 188-200) we examine the relationship 
between cognitive abilites of people with PWS and acadenic achievement in reading, writing and arithmetic, using a comparison group of people who had been thought to have PWS, but were found not to have the disorder, together with a group of people with learning disabilities not caused by PWS. The ains of this study were to investigate whether attainments which people with PWS might have been expected to achieve on the grounds of their $1 \mathrm{Q}$ were achieved, and to ask which factors might be associated with any underachievement.

\subsection{Malladaptive behaviour}

In the 1960 s a number of articles started to appear regarding the behavioural aspects of PWS, including reports noting maladaptive behaviours such as temper tantrums and "states of depression" (Zellweger \& Schneider, 1968; Dunn 1968; Hall \& Smith, 1972; Holm \& Pipes, 1976). Curfs (1992) and later Bonghgraef et al. (1990) noted that temper tantrums and rage reactions were frequently reported in association with the condition. Borghgraef et al. reported that as toddlers their patients with PWS were cheerful and cordial, but that at the age of 3 to 5 years violent crying tantrums first appeared, often related to the withholding of food. During puberty depressive symptoms became apparent, with rapid swings of behaviour, associated with occasional sudden acts of inappropriate violence.

More structured surveys of behavioural abnormalities using standardised instruments followed in the 1980s. Whitman \& Accardo (1987) used the Survey Diagnostic Instrument (a 134 item questionnaire screening for DSM-II criteria-based diagnostic categories) and additional items on 35 children, adolescents and young adults. They asked the parents to fill in the questionnaire, which was a structured, standardised assessment, but which did not include a standardised scoring system. Whitman \& Accardo found that 54\% of adolescents with PWS studied had a definite or probable diagnosis, compared to around $19 \%$ for the general population. The diagnoses were predominanty of neurotic disorders, including obsessive behaviours, although suspiciousness, "strange" ideation, and auditory and visual hallucinations were also reported for a minority of subjects.

Greenswag (1987) described a postal questionnaire survey, designed for caregivers (most were parents) of 232 adult members of the Prader-Willi Syndrome Association in the USA and of contacts made through the 23 genetics clinics in the USA. People with questionable diagnoses were excluded from her analysis. The most common behavioural characteristics, as reported by carers, were: "slow-moving, good natured, stubborn, sleepy, belligerent (food related), irritable, impulsive, lazy, belligerent (non-food related), antisocial with peers, moody, antisocial with family, and physically aggressive", which had ratings from 3.6 ("slow moving") to 2.2 ("physically aggressive") on a four-point scale from 1 (never observed) to 4 
(frequently observed). Analysis of variance was used to compare weight with behavioural ratings and Greenswag found that the heavier adults were more frequently rated $(\mathrm{p}<0.05)$ as "lazy, sleepy, antisocial with their families and slow moving".

Clarke et al. (1989) sent a postal questionnaire survey to 111 people with PWS over the age of 12 contacted through the Prader-Willi syndrome Association (UK). Seventy replies were received and, excluding those under 16 years, 32 female and 29 male patients were included. Common behavioural problems were found to include deliberate picking of sores (sometimes resulting in persistent sores), food stealing, temper tantrums, and a variety of sleep problems.

Prior to our study on maladaptive behaviour in 1996 (Clarke et al; Appendix 1) few studies had used screening instruments or standardised measures of behavioural abnormalities or personality among individuals with PWS. Most studies merely reported clinical impressions, or used questionnaires. Although clinical impressions or specially designed questionnaires may be important during the initial stages of information gathering (for instance to identify areas of concern), they do not allow for comparisons to be made between groups, and or to quantify any abnormalities. Studies by Whitman and Accardo, using the Survey Diagnostic Instrument, and by Curfs et al. (1991) of 27 children and adolescents with PWS form an exception. Curfs et al. (1992) commented that the "absence of systematically gathered data on behavioral characteristics, including social-emotional functioning and personality characteristics is striking". We therefore set out to use standardised tests to discover whether behaviour found in PWS is different from that of other patients with a learning disability. We compared maladaptive behaviours of adults with PWS and those of adults in community residential facilities for people with learning disabilities (Clarke et al. 1996). The Aberrant Behavior Checklist $(\mathrm{ABC})$, a widely used standardised instrument was used to compare the two groups. We looked at adults from the age of 16 years and older because there is some evidence that maladaptive behaviour changes with age or development. Other studies of PWS have used this convention and this allowed results to be compared. The PWS sample was recruited through the PWS Association (UK) and colleagues in fields such as psychiatry, psychology and social work. Of the 30 people with PWS, 21 had a deletion, two had maternal disomy, and one had additional material on the long arm of chromosome 15. Six of the 30 had a firm clinical diagnosis of PWS, but no diagnosable genetic abnormality (the diagnoses were made without the use of behavioural criteria). The Community Residential Facility (CRF) Contrast Group consisted of 30 people who were resident in or who received respite care in residential Social service or volun tary sector facilities. Those in the contrast group were not recruited from Community Residential Facilities which provide specialist care for people with severe sensory impairments or autistic disorders. The groups were matched for age (within 18 months), sex 
Table 4. Maladaptive behaviour: mean ABC factor scores for the 30 PWS subjects and 30 contrast subjects resident in community residential facilities.

\begin{tabular}{lllllll}
\hline Factors & I & II & III & IV & V & Total \\
\hline PWS subjects & 18.8 & 6.8 & 0.96 & 9.3 & 3.93 & 39.93 \\
CFF subjects & 6.2 & 6.6 & 2.4 & 9.2 & 2.2 & 26.6 \\
P (Mann-Whitney test) & $<0.001$ & NS & NS & NS & $<0.05$ & NS \\
\hline
\end{tabular}

$\mathrm{I}=$ irritability, agitation; III = lethargy, withdrawal; III = stereatypic behaviour; $I V=$ hyperactivity, non-compliance; and $W$ - inappiropriate speech.

From: Clarke D., Boer H., Chung M.C. Sturmey P. \& Webb T. (1996) Maladaptive Behaviour in Prader-w"lli Syndrome in adult tife. Journal of Intellectwal Disability Research 40, 159-65.

and degree of mental retardation (using ICD 10 criteria). Maladaptive behaviour was assessed using the Aberrant Behaviour Checklist developed by Aman ef al. $(1985 \mathrm{a}, \mathrm{b})$. This checklist consists of a 58 -item structured interview with a carer and requires a rating of maladaptive behaviours from zero (no problem) to three (the problem is severe in degree). The original factor analysis of these items revealed a 5 -factor solution, which was judged to be both psychologically meaningful and psychometrically accurate. The $\mathrm{ABC}$ was used because its reliability and. validity in assessing mallaptive behaviour among people with cognitive impairment has been demonstrated ref (Aman et al. 1985a, b; Newton \& Sturmey 1988). The factors or subscales are: I = irritability, agitation; II = lethargy, withdrawal; III = stereotypic behaviour; $\mathrm{IV}=$ hyperactivity, non-compliance; and $\mathrm{V}=$ inappropriate speech. We used both factor and item scores to compare the two groups. Table 4 shows the Mean ABC factor scores for the 30 PWS subjects and the 30 contrast subjects.

The PWS subjects scored significantly higher in factor I (irritability, agitation) and factor V (inappropriate speech). They scored lower in factor III (stereotypic behaviour) but this was not significant. The PWS group also had significantly higher scores for items relating to: tempers, impulsiveness, non-compliance, mood disotder inactivity, and repetive speech. The PWS sample had mean scores above 1.0 for 17 items, whereas the CRF group had no ABC items with a mean score exceding 1. Younger PWS subjects had marginally higher scores than older subjects. Factor scores did not differ significantly between men and women with PWS. We also compared the 10 subjects most overweight for height with the 10 stabjects least overweight for height. The overweight group had significantly higher scores for factor I (imitability, agitation) and IV (hyperactivity, noncompliance) compared to the 10 subjects who were least overweight. In conclusion these results support previous research in delineating behaviours which are particularly problematic for people with PWS, but PWS is associated with secondary features (such as obesity and sleep disorders) which may influence 
maladaptive behaviours. These are not easily controlled for. Sleep disorders, for instance, may contribute to daytime irritability and loss of temper. Other aspects which may modify the type or extent of maladaptive behaviour include the age at which dietary restrictions are imposed and perhaps the consistency with which the dietary restrictions are imposed). Comparisons between factor scores may mask differences relating to specific items within factors. This is especially retevant when factors combine items relating to different consuructs such as lethargy and social withdrawal; lethargy, for example, may be a problem for many people with PWS, but social withdrawal is not.

In two further studies we used standardised instruments to compure the behaviour of patients with PWS with patients with other genetic syndromes, published as "Clarke D.J. \& Boer H. (1998) Problem Behaviors Associated with Deletion Prader-Willi, Smith-Magenis, and Cri Du Chat Syndromes. American Jommal on Mental Retardation. 103, 264-271," and "Boer H. \& Clarke D. (1999) Development and behaviour in genetic syndromes: Prader-Willi Syndrome. Jommal of Applied Research in Intellectual Disabilities. 12, 294-301". The results are presented as chapter $4 a$ and chapter $4 b$, respectively. This latest study should be read in conjunction with Boer H., Langton J. \& Clarke, D. (1999) Development and behaviour in genetic syndromes: Rubinstein-Taybi Syndrome, Jommal of Applied Researh in Intellectual Disabilities. 12, 302-7" (appendix 2), as these were published as subsequent articles. Smith-Magenis, Cri Du Chat, and Rubinstein-Taybi syndromes were chosen as they are all caused by chromosomal (micro)deletions, in order to allow comparison with PWS, as most cases of PWS are also caused by deletions.

\subsection{Compulsive and ritualistic behaviours}

Whitman and Accardo (1987) reported that a significant number of people with PWS had "compulsive neurosis". They reported that statements about the patients such as "Worries about own things being stolen", and "argues a lot" were rated as "often" or "very true" in $43 \%$ and $69 \%$ respectively of adolescents with PWS. Our study on maladaptive behaviour (Clarke et al. 1996; see Appendix 1) showed scores on the Aberrant Behaviour Checklist on items such as "nepetitive speech" and "repeats a phrase over and over" which were significantly more common than in a matched group of people living in a community residential facility.

In 1996 Dykens et al. described the obsessions and compulsions of a group of 91 children and adults with PWS, and compared 43 adults with PWS with an ageand sex-matched group of people with obsessive-compulsive disorder (OCD). but who did not have a learning disabilicy. Parents and caregivers of the PWS 
group were asked to complete an informant version of the Yale-Brown Obsessive Compulsive Scale, adapted for use for people with learning disability. Those with OCD were asked to complete the self-report version of the Yale-Brown Obsessive Compulsive Scale. In the PWS group the symptoms "hoarding" and "need to know" were seen in over half the sample and "ideas of symmetry and exactness" and the "need to rewrite, order and arrange things" were seen in over a third, with about a quarter showing "excessive showering, toileting and grooming". The authors comment that telling or asking was often characterised by persistent questioning or commenting on a narrow range of topics, and that many informants noted that, when they tried to interrupt compulsive behaviours, this led to increased anxiety, argumentativeness and temper tantrums. Comparing the two groups, Dykens et al. found that the PWS and OCD groups had comparable numbers of compulsions, but that the OCD group were more likely to have obsessions. Relatively few people in the PWS group had symptoms of cleaning, contamination, or checking. The authors comment that as people with PWS scored highly on a factor including hoarding, repeating rituals and concerns with symmetry, exactness, ordering and arranging, it may be that they display one dimension of the OCD phenotype. The PWS group had compulsions that were similar to rituals and repetitive behaviours that are common in preschool children without learning disabilities. A limitation of the study by Dykens et $a$ l. is that it relied on ratings by carers or patients themselwes, rather than by cliniciars, and, in comparing the two groups, the results of one group (PWS) was based on informant ratings, and of the other group (OCD) on ratings by the individuals themselves. A further limitation is the lack of a control group matched for learning disability. Warnock and Kestenbaum (1992) suggested that skin-picking behaviours in PWS may be a variant of obsessive-compulsive disorder (however, see chapter 8.3 and 8.4), and treated two patients successfully with the selective serotonin reuptake inhibitor antidepressant fluoxetine. Feurer et al. (1998), in a factor analytic study, found that skin picking did not appear on the same factor as compulsivity on the Compulsive Behavior Checklist, implying that skin picking in PWS is not part of any cluster of obsessive-compulsive symptoms.

The fact that obsessive-compulsive symptoms may improve following treatment with anticlepressants that act on serotonin such as clomipramine and the serotonin reuptake inhibitors, suggest that the serotonin system is involved in the pathogenesis of OCD. Akefeldt ef al. (1998) compared the concentration levels of monoamine metabolites in the cerebrospinal fluid of 13 children and adolescents with PWS and in a control group, and found evidence for increased serotonin tumover in PWS, and for decreased synaptic serotonin transmission. The authors comment that many of the symptoms and behaviours seen in PWS may be connected with serotonergic activity. 
Dimitropoulos et al. (2000) comment that a clinical diagnosis of OCD is not often diagnosed in PWS, and that it appears that the obsessive-compulsive symptoms seen in people with PWS may involve different mechanisms.

The emergence of obsessive-compulsive symptoms from our study on Maladaptive Behaviour in Prader-Willi Syndrome (1996; Appendix 1), and the fact that no population-based research, and no research with an appropriate control group had been done, led to our research presented in chapter 6 , published as "Clarke D. J., Boer H., Whittington J., Holland A., Butler J. \& Webb T. (2002) Prader-Willi syndrome, compulsive and ritualistic behaviours: the first population-based survey. British Joumal of Psychiatry 180, 358-62". Our aim was to establish the nature and prevalence of the Obsessive-compulsive symptoms in PWS.

\subsection{Prader-Willi syndrome and psychosis}

Kollrack and Wolff (1966) reported what is thought to be the first description of a psychotic patient with PWS in the literature. They described a young man who became agitated, and noted that "Eine beruhigung durch freundsshaftlichen Zuspruch war in keiner Weise möglich. Er redete ununterbrochen mit hochrotem Kopf weiter und stiess zwischendurch Drohungen aus wie 'Wenn ich die erwische, die schlage ich, denen werde ich's zeigen" " (see Clarke er al. 1998, chapter 7). He had delusions that the police were after him for having stolen a hare. The patient needed hospitalisation, recovered, but later needed a further admission. He is likely to have suffered from an affective psychosis. Bray et al. (1983) reported that two patients out of their series of 40 adults with PWS had serious mental problems and that they needed admission to locked psychiatric wards. These patients had suffered from "severe withdrawal from interpersonal interaction and apparent regressive behavior", but both later made a spontaneous remission. In their questionnaire study Whitman and Accardo (1987) reported that carers responded that auditory hallucinations occurred sometimes in 12 out of 35 adolescents with PWS. In 1993 David Clarke reported three patients with PWS and psychotic disorders, and Clarke et al. presented a further case in 1995. Jerome (1993) presented a 31-year-old woman with PWS and a rapid cycling mood disorder. Ewald et al. (1994) described a woman with PWS who had wo episodes of psychosis. They found no evidence of a linkage between two GABA subtype markers in the $15 \mathrm{q} 11 \mathrm{q} 12$ region and manic-depressive illness, and commented that the fact that psychosis is not an obligate feature of PWS may be explained if a gene involved in the aetiology of psychosis is located at one of the borders of the chromosome $15 \mathrm{q} 11 \mathrm{q} 13$ region. Further cases were presented by Whittaker et al. (1997) and Watanabe et al. (1997). 
In order to attempt to establish whether psychotic symptoms were part of the PWS behavioural phenotype, an international collaboration was proposed, and we presented six further cases of PWS and psychotic symptoms (See chapter 6a: Clarke D., Boer H., Webb T., Scott P., Frazer S., Vogels A., Borghgraef M. \& Curfs L.M.G. (1998) Prader-Willi syndrome and psychotic symptoms. Case descriptions and genetic studies. Jowmal of Intellectwal Disability Research $\mathbf{4 2}$, 440-50). This study, together with three other studies appearing in the same edition of the Journal of Intellectual Disability Research (Clarke, 1998; Verhoeven et al. 1998; Beardsmone et al. 1998) made it likely that our hypothesis that there is a non-chance relationship between PWS and psychoses was correct. In order to confirm this we examined whether PWS was associated with psychosis in a population study. The results of this study are presented in chapter $6 b$ (Boer H., Holland A., Whittington J., Butler J., Webb T. \& Clarke D. (2002) Psychotic illness in people with Prader-Willi syndrome due to chromosome 15 matemal uniparental disomy. Lancer 359, 135-6).

\subsection{Behaviour and event related brain activity}

In view of our finding that there were striking differences between the genetic subtypes, such as the heightened vulnerability of PWS patients with UPD for affective psychoses (see chapter $6 \mathrm{~b}$ ), together with the dissimilar cognitive profiles (see chapter 1.5) we examined whether such differences were supported by psycho-physiological studies.

Event-related brain activity of event-related potentials (ERP) can be extracted by recording an electro-encephalogram (EEG) while the subject is performing a task. The ERP therefore reflects the cognitive processing in real time. One of the tasks that is often used is the oddball task, typically by presenting a series of stimuli with a low, and with a high probability of occurring during the task while the subject is asked to respond to the rare (low probability of occurring) stimulus by pressing a button. This rare stimulus then results in a positive cognitive potential which occurs around $300 \mathrm{~ms}$ after the stimulus onset. This is called the P300. The ratio between low and high probability stimuli is usually about $1: 5$. Stauder et al. (2002) compared ten adults with PWS to a control group of ten people without a leaming disability. The ten people with PWS all had a genetic confimation of the syndrome, but no details of the genetic subtypes were given. Both groups completed the tasks successfully although the PWS group made more errors, and had fewer "target hits" in the visual oddball task. Stauder et al. found that there was a deflation in the P3 component of both the visual and the auditory oddball tasks in the PWS group. The auditory modality was more affected than the visual modality, and there was evidence for short-term memory impaiment in PWS. 
The study confirmed earlier cognitive findings in PWS (see chapter 1.5), and confirmed the importance of studies combining the behavioural and electro-physiological approach. The limitation of this study was that it did not allow for a comparison between the disomy and UPD subgroup to be made, and we therefore applied for ethical approval for a study comparing the two groups in an. ERP study. The results are presented in chapter 7a (Stauder J.E.A., Boer H., Gerits R.H.A., Tummers A., Whittington J. \& Curfs L.M.G. Marked differences in behavioural phenotype between parental deletion and matemal uniparental disomy in Prader-Willi syndrome: Behaviour and event related brain activity to a response inhibition task. Submitted) and in chapter $7 \mathrm{~b}$ (Boer H., Stauder J.E.A., Gerits R.H.A., Tummers A, Whittington J., Webb T. \& Curfs L.M.G. Prader-Willi syndrome: Behavioural. differences between deletion and disomy subtypes. Submitted).

\section{References}

Ákefeldt A., Anvret M., Grandell U, Nordlinder R. \& Gillberg C. (1995) Parental exposure to hydrocarbons in Prader-Willi syndrome. Developmental Medicine and Child Neurology 37, $1101-9$.

Åkefeldt A., Gillberg C. \& Larsson C. (1991) Prader-Willi syndrone in a Swedish rural county: Epidemiological aspects. Developmental Medicine and Child Neurology 33, 715-21.

Åkefeldt A., Ekman R., Gillberg C. \& Månsson J.E. (1998) Cerebrospinal fluid monoamines in Prader-Willi syndrome. Biological Psychiarty 44, 1321-8.

Aman M.G., Singh N.N., Stewart A.W. \& Field C.J. (1985a) The Aberrant Behavior Checklist: a behavior rating scale for the assessment of treatment effects. American Jourtal of Mental Deficiency 89, 485-91.

Aman M.G., Singh N.N., Stewart A.W. \& Field C.J. (1985b) Psychometric characteristics of the Abernant Behavior Checklist. American foumal of Mental Defidency 89, 492-502.

Angelman H. (1965) "Puppet" children: a report on three cases. Developmental Medicine and Child Neurology 7, 681-8.

Beardsmore, A., Dorman T., Cooper S.-A. \& Webb T. (1998) Affective psychosis and Prader-Willi syndrome. Joumal of Infellectual Disability Research 42, 463-71.

Boer H., Clarke D., Webb T., Holland A. \& Curfs L.M.G. (1998) Het Syndroom van Prader-Willi: van Gen naar Gedrag (Prader-Willi Syndrome: From gene to behaviour). Tijdschiff voor Psychiatrie (Dutch Journal of Psychiatry) 40, $108-14$.

Boer H. \& Clarke D. (1999) Development and behaviour in genetic syndromes: Prader-Willi Syndrome. Journal of Applied Research in Intellectual Disabilities 12, 294-301.

Boer H., Langton J \& Clarke D. (1999) Development and behaviour in genetic syndromes: Rubinstein-Taybi Syndrome. Joumal of Applied Research in Intellecrual Disabilities 12, 302-7. 
Boer H., Holland A., Whittington J., Butler). Webb T. \& Clarke D. (2002) Psychotic illness in people with Prader-Willi syndrome due to chromosome 15 matemal uniparental disomy. Lancet $359,135-6$

Boer H., Stauder J.E.A., Gerts R.H.A., Tummers A., Whittington J., Webb T. \& Curfs L.M.G. Pradet-Willi syndrome: Behavioural differences between deletion and disomy subtypes. Submitted.

Borghgraef M., Fryns J.P. \& Van den Berghe H. (1990) Psychological profile and behavional characteristics in 12 patients with Prader-Willi syndrome. Genetic Couselling 38, 141-50.

Boyd S.G., Harden A. \& Patton M.A. (1988) The EEG in early diagnosis of the Angelman (Happy Puppet) syndrome. European Joumal of Pediatrics $147,508-513$.

Bray G.A., Dahms W.T., SwendloffR.S., Fiser R.H., Atkinson R.L. \& Carrel R.E. (1983) The Prader-Willi syndrome: a study of 40 patients and a rewiew of the literature. Medicine (Ballimore) $62,59-80$.

Buiting K., Saitoh S., Gross S., Ditrich B., Schwartz S., Nicholls R.D. \& Horsthemke B. (1995) Inlherited microdeletions in the Angelman and Prader-Willi syndromes define an imprinting cente on human chromosome 15. Natwe genetics 9, 395-400.

Buiting K., Gross S., Lich C., Gillessen-Kaesbach G., el-Marri O. \& Horsthemke B. (2003) Epimutations in Prader-Willi and Angelman syndromes: a molecular study of 136 patients with an imprinting defect. American joumal of hmman genetios 72, 571-7.

Burd L., Vesely B., Martsolf J. \& Kerbeshian J. (1990) Prevallence study of Prader-Willi Syndrome in North-Dakota. American Joumal of Medical Genetics 37, 97-9.

Butter J. V., Whittington J.E., Holland A.J., Boer H., Clarke D. \& Webb T. (2002) Prevalence of, and risk factors for, physical ill-health in people with Prader-Willi syndrome: a population-based study. Developwental Medicine G Child Neurology 44, 248-55.

Butler M.G. (1990) Prader-Willi syndrome: current understanding of cause and diagnosis. American Joumal of Medical Genetis 35, 319-332.

Buter M.G., Bittel D.C., Kibiryeva N., Talebizadeh Z. \& Thompson T. (2004) Behavional differences among subjects with Pader-Willi syndrome and type I or type II deletion and maternal disony. Pediatrics 113, 565-73.

Carpenter P.K. (1994) Prader-Willi syndrome in old age Joumal of Intellectwal Disability Rescard $38,529-31$.

Cassidy S.B., Schwartz S. (1998) Prader-Willi and Angelman syndromes: Disorders of genomic inprimang. Medicine 77, 140-51.

Clarke D.J., Waters J \& Corbett A. (1989) Adults with Prader-Willi syndrome: abnormalities of sleep and behaviour. Jommal of the Royal Society of Medicine $82,21-4$.

Clarke D.J. (1993) Prader-Willi syndrome and psychoses. British Joumal of Psychiony 163 , $680-4$

Clarke D.J., Webb T. \& Bachmann-Clarke J.P. (1995). Prader-Willi syndrome and psychotic symptoms: report of a further case. Irish Joumal of Pyohologial Medicine 12, 27-9.

Clarke D. Boer H. \& Webb T. Genetic and behavioural aspects of Prader-Willi Syndrome. (1995) Mestal Handiap Researh 8, 38- 53 . 
Charke D., Boer H., Chung M.C., Stumey P. W Webb T. (1996) Maladaptive Behaviour in Prader-Willi Syndrome in adult life. Joumal of lntellewal Distbing Resenth 40, $159-65$.

Clarke D.J. \& Boer H. (1998) Problem Behaviors Associated with Deterion Prader Willi. Srnith-Magenis, and Cri Du Chat Syndromes. Amerian Jommat on Meud Retardotion 103, 264-71.

Clarke D., Boer H., Webb T., Soot P. Frazer S., Vogels A., Borghgraef M. A Curf L.M.G. (1998) Prader-Willi syndrome and psychotic symptoms 1. Case deseriprions and generic studies. Jouma of Intellectual Disability Research 42,440-50.

Clarke D. (1998) Prader-Willi syndrome and psychotic symptoms: 2. A preliminary study of prevalence using the Psychopathology Assessment for Adults with Developmental Disability checklist. Jowmal of Intellowal Disability Researh 42, 445-54.

Clarke D.J., Boer H., Whittington J., Holland A., Butlex J. \& Webb T. (2002) Prader-Will syndrome, compulsive and ritulistic behaviours: the first population-based survey. British Joumal of Psychiatry $180,358-62$.

Curfs L.M.G., Wiegers A.M., Sommers J.R.M., Borghgraef M. \& Fryns M. (1991) Strengths and weaknesses in the cognitive profile of youngsters with the Prader-Will syndrone. Clintial Genctios 40, 430-4.

Curfs L.M.G. \& Fryns J.P. (1991) Plader-Will syndrome: a review with special attention to the cognitive and behaviour profile. In Psychosoial Aspects of Genefics. Wiley and Sons, New York

Curfs L.M.G. (1992) Psychological profile and behavional characteristics in the Prader-Willi syndrome. In: Prader-Will Syndrome and Other Chronosome 15 gdetion Disorders (ed S. 13. Cassidy). Springer-Verlag, Berlin.

Curfs L.M.G., Verhulst F.C. \& Fryns J.P. (1991) Behavioural and emotional problems in youngsters with Prader-Willi syndrome. Genetic Counseling 2, 33-41.

Dimitropoulos A., Feurer I. D., Roof E., Stone W.L., Butler M.G., Sutcliffe J. \& Thompson T. (2000). Appetitive behavior, compulsivity, and neurochemistry in Prader-Willi

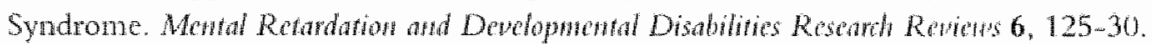

Donlon T.A. (1988) Similar molecular deletions on chromosome $15 q 11.2$ are encountered in both the Prader-Willi and Angelman syndromes. Human Genetio $80,322-8$.

Dum H.G. (1968) The Prader-Labhart-Willi syndrome; Review of the literature and report of nine cases. Acta Paedinatioa Sadodinatia Suppl. 186, 1-38.

Dykens E.M., Hodapp R. M., Walsh K. \& Hash L.J. (1992) Profies, correlates, and trijectories of intelligence in Prader-Willi syndrome. Journal of the Amerian Aardeny of Child and Adolescent Psydiany 31,1125-30.

Dykens E.M., Lecknuan J.F., \& Cassidy S.B. (1996). Obsessions and compulsions in Prader-Willi syndrome. Joumal of Child Psychology and Psyliatry 37, 995-1002.

Dykens E.M. (2004) Maladaptive and compulsive behavior in Prader-Willi syndrome: New insights from older adults. Amerian Jownal on Mental Retardation 109. 142-53.

Ewald H. Mors O. Flint T. Kuuse T.A. (1994) Linkage analysis between manic-depresive illness and the region on chromosome 15 q involved in Prader-Will syndrome, including two GABA(A) receptor subrype genes. Human Heredity 44, $287-94$. 
Feurer D., Dimitropoulos A., Stone W.L., RoofE. Butler M. G. Thompson T. (1998) The latent wariale structure of the Compulsive Behawour Checklist in people with Prader-Willi syndrome. Jownal of intellectual disability researh 42, 472-80.

Gallagher R.C., Pils B. Alballwi M. \& Francke U. (2002) Evidence for the role of PWCR 1/HBII-85 C/D box small nucleolar RNAs in Prader-Willi syndrome. American joumal of human gentetics 71, 669-78.

Greenswag L.R. (1987) Adults with Prader-Willi syndrome: a survey of 232 cases. Developmental Medicine and Child Neutology 29,637-48.

Gunay-Aygun M., Schwartz S., Heeger S., O-Riordan M.A. \& Cassidy S.B. (2001) The changing purpose of Prader-Willi syndrome clinical diagnostic criteria and proposed revised criteria. Pediatrics: 108 , e92.

Hall B.D. \& Smith D.W. (1972) Prader-Willi syndrome: a resumé of 32 cases including an instance of affected cousins, one of whom is of nomal stature and intelligence. Joumal of Pediatrics 81, 286-93.

Harris J.C. \& Allen R.P. (1996) is excessive daytime sleepiness characteristic of Prader- Willi syndrome? The effects of weight change. Archives of Pediatrics $\&$ Adolescent Medicine 150 , $1288-93$

Helbing-Zwanenburg B., Kamphuisen H.A.C. \& Mourtazaev M.S. (1993) The origin of excessive daytime slepeness in the Prader-Willi syndrome. Joumal of Intellectual Disability Researd 37, 533-41.

Holland A.J., Treasure J., Coskeran P. \& Dallow, J. (1995) Characteristics of the eating disorder in the Prader-Willi syndrome: implications for treatment. Jowmal of Intelletual Disability Reseawd 39, 373-81.

Holland A.I. \& Wong J. (1999) Genetically determined obesity in Prader-Willy syndrome: The ethics and legality of treatment. Jourtal of Medical Ethics 25, 230-6.

Holm V.A., Cassidy S.B., Butter M.G., Hanchetr J.M., Greenswag L.R., Whitman B.Y. \& Greenberg F. (1993) Prader-Willi syndrome: consensus diagnostic criteria. Pediatrics 91 , $398-402$.

Holn V.A. \& Pipes P.L. (1976) Food and children with the Prader-Willi syndrome. American Joumal of Disenses of Childrew 130,1063-7.

Kishino T., Lalande M. \& Wagstaff J. (1997) UBE3A/E6-AP mutations cause Angelman syndrome. Nature gencicic 15, 70-3.

Kollack H.W. \& Wolff D. (1966) Paranoid-halluxinatorische Psychose bel Pader-Labhart-Willi-Fancon syndrome. Acha Paedopsychiatria 33, 309-14.

Kuwano A., Mutiranguta A., Dittrich B., Buiting K., Horsthemke B., Saitoh S., Niikawa N., Ledbeter S.A., Greenberg F., Chinatit A.C. \& Ledbetter D.H. (1992) Molecular dissection of the Prader-Willi/Angelman syndrome region (15q11-13) by YAC cloning and FISH analysis. Hown wolecwlar gentics $1,417-25$.

Lachman H.M., Kelsoe J.R., Remick R.A., Sadovnick A.D. Rapaport M.H., Lin M. Pazur B.A., Roe A.M.A. Saito T. \& Papolos D.F. (1997) Linkage studies suggest a possible locus for bipolar disorder near the velo-cardio-facial syndrome region on chromosome 22 . Ameriran Joumal of Medical Generics Newropsychiatric Genetics 74, 121-8. 
Laurance B.M., Brito A. Wilkinson J. (1981) Prader-Willi syndrome after age 15 years. Ardwives of Disease in Childhood $56,181-6$.

Ledbetter D.H., Riccardi V.M., Airhart S.D., Strobel R.J., Keenan B.S. \& Crawtond J.D. (1981) Deletion of chromosome 15 as a cause of Prader-Will syndrome. Now England Jowmal of Medicine 292, 63-6.

Matsuura T., Sutcliffe J.S., Fang P., Galjaard R.J. Jiang Y. H., Benton C.S., Rommens J M. B* Beaudet A.L. (1997) De novo truncating mutations in E6-AP ubiquitin-protein ligase gene (UBE3A) in Angelman syndrome. Nature getwetics 15, 74-7.

Murphy K.C. Jones L.A. \& Owen M.J. (1999) High ates of schizophrenia in adults with velom-cardio-facial syndrome. Ardives of General Psychatry 56,940-5.

Newton, I.T. \& Stumey, P. (1988). The Aberant Belzwior Checklist: A British replication and extension of psychometric properties. Jotwhal of Menal Deficiency Reseam 32 , $87-92$.

Nicholls R.D. \& Knepper J.L. (2001) Genome organization, function, and imprinting in Prader-Willi and Angelman syndromes. Anmul Review of Genomics and Hwhon Genetios 2 . 153-75.

Nicholls R.D., Knoll.J.H., Butler M.G., Karam S. \& Lalande M. (1989) Genetic imprinting suggested by matemal heterodisomy in nondeletion Prader-Willi syndromia. Narture 342. $281-5$.

Nicholls R.D. (1993) Genomic imprinting and unparental disomy in Angetman and Prader-Willi syndromes: a review. Anerian joumat of medical genctios 46, 16-25.

Özçelik T., Leff.S., Robinson W., DonlonT, Lalande M., Sanjines E., Schinzel A. \& Francke U. (1992) Small nuclear ribonucleoprotein polypeptide N (SNRPN), an expressed gene in the Prader-Willi syndrome critical region. Nature genetics: 2, 265-9.

Papolos D.F., Faedda G.L., Veit S., Goldberg R., Morrow B., Kucherlapati R. \& Shprintzen R.J. (1996) Bipolar spectrum disorders in patients diagnosed with velo-cardio-facial syndrome: Does a hemizygons deletion of chromosome $22 \mathrm{q} 11$ result in bipolar affeative disorder? American Journal of Psychiary 153, 1541-7.

Pembrey M., Fennell S.J, wan-den-Berghe J. Fitchet M. Summers D., Butler L., Clarke C.. Griffith M., Thompson E. \& Super M. (1989) The association of Angchman's syndrome with deletions within 15q1 l-13. Joumal of Medical Generis 26,73-7.

Prader A. Labhart A. \& Willi H. (1956) Ein Syndrom von Adipositas, Kleinwuchs, Kryptorchismus und Oligophrenie nach myatonieartigen Zustand im Neugeborenalter.

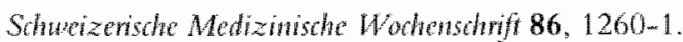

Richdale A.L., Cotton S. \& Hibbit K. (1999) Sleep and behaviour disturbance in Prader willi syndrome: a questionnaire study. Joumal of intellectual disability rocard 43,380-92.

Roof E., Stone W. MacLean W., Feurer I.D., Thompson T. \& Butler M. C. (2000) Intellectual characteristics of Prader-Willi syndrome: comparison of genetic subtypes. Jowrnd of Intelleowal Disability Researh 44, 25-30.

Schrander-Stumpel C.Th.R.M., Curfs L.M.G., Sastrowijoto P., Cassidy S.B., Schrander J. I. \& Fryns J.P. (2004) Prader-Willi Syndrome: Causes of Death in an International Series of 27 Cases. American Jownal of Medical Genetics. 124, 333-338. 
Stauder J.E., Brinkman M.J. \& Curfs L.M.G. (2002) Multi-modal P3 deflation of event-related brain activity in Prader-Will syndrome, Newroscience Letters 327, $99-102$.

Stauder J.E.A., Boer H., Gerits R.H.A., Tummers A, Whitrington J. \& Curfs L.M.G. Differnces in behavioural phenotype between parental deletion and matemal uniparental disomy in Prader-Willi syndrome. Submitted.

Stevenson D.A., Anaya T.M., Clayton-SmithI., Hall B.D., Van Allen M.I., Zori R. T., Zackai E.H., Frank G. \& Clericuzio C.L. (2004) Unexpected death and critical illness in Prader-Willi syndrome: report of ten individuals. Amerian Jowmal of Medial Genetios $124 \mathrm{~A}$, $158-64$.

Van-Hooten R.H. Widdershoven G.A., wan-den-Borne H.W. \& Curf L.M.G. (2002) Autonomy and intellectual disability: the case of prevention of obesity in Prader-Will syndrome. Joumal of Intellectual Disability Research 46, 560-8.

Vela-Bueno A., Kales A., Soldatos C.R., Dobladex-Blanco B., Campos-Costello J., Espino-Hurtado P. \& Olivan-Palacios J. (1984) Sleep in the Prader-Willi syndrome. Clinical and polygraphic findings. Archives of Neurology 41, 294-6.

Verhoeven W.M.A., Curfs LM.G. \& Tuinier S. (1998) Prader-Willi syndrome and cycloid psychoses. Joumal of Intellectual Disability Research 42, 455-62.

Vogels A, Van-Den-Ende J, Keymolen-K., Mortier G., Devriendt K., LegiusE. \& Fryns J.P. (2004) Minimum prevalence, birth incidence and cause of death for Prader- Willi syndrome in Flanders. European jowrmal of humam genetics 12, 238-40.

Watanabe H. Ohmori O. \& Abe K. (1997) Recurrent brief depression in Prader-Willi syndrome: A case report. Psychiatric Geretios 7, $41-4$.

Warnock J.K. \& Kestenbaum, T. (1992) Pharmacologic treatment of severe skin-picking behaviors in Prader-Willi syndrome: two case reports Archives of Dematology 128, 1623-5.

Webb T., Whittington J., Clarke D., Boer H., Butler J. \& Holland A. (2002) A study of the influence of different genotypes on the physical and behavioral phenotypes of children and adults ascertained clinically as having PWS. Clinical genetics 62, 273-81.

Whitman B.Y. \& Accardo P. (1987) Emotional symptoms in Prader Willi syndrome adolescents. Amerian Joumal of Medical Genetic 28,897-905.

Whituker. F., Cooper C., Hanington R. C. \& Price D.A. (1997) Prader-Willi syndrome and acute psychosis. Intemational Jownd of Pychiatry in Clintal Pratice 1, 217-9.

Whittington J.E., Holland A.J., Webb T., Butler J. W., Clarke D.J. \& Boer H. (2001) Population prevalence and estimated birth incidence and mortality rate for people with Prader-Willi Syndrome in one UK Health R egion. Jownd of Medical Genetios 38, 792-8.

Whittington J.E., Holland A.J., Webb T., ButlerJ. V., Clarke D.J. \& Boer H. (2002) Relationship between clinical and genetic diagnosis of Prader-Willi syndrome. Jommal of Medrad Genctics $39,926-32$.

Whittington J. Holland A., Webb T., ButlerJ, Clarke D. \& Boer H. (2004) Cognitive abilities and genotype in a population-based sample of people with Prader-Willi syndrome. Joumal of intellectwal disabitity peseard $48,172-87$. 
Whittington J., Holland A., Webb 'T., Butler J., Clarke D. B Boer H. 2004) Academic underachicvement by people with Prader-Will syndrome. Jownd of lntelletwal Disability Reserath: 48, $188-200$.

Williams C.A., Zoti R.T., Stone J.W., Gray B.A., Cantu E.S. \& Ostrer H. (1990) Matemal origin of 15q11-13 deletions in Angehman syudrome suggests a role for genomic imprinting. Amerian joumat of medial genetios 35, 350-3.

Zellweger H. \& Schneider HJ. (1968) Syndrome of hypotonia-hyponentambyogonadism-obesity (HHHO) or Prader-Willi syndrome. Americanjonmal of disenses of dildren $115,588-98$.

ZellwegerH. \& Soper R.T. (1979) Lesyndrome de Prader-Willi dans les Amériques. Médicin at Hyoure $37,3338-45$. 

Chapter 2

\section{Population prevalence and estimated birth incidence and mortality rate for people with Prader-Willi syndrome in one UK Health Region}

Published ats:

Whittington J.E., Holland A.J., Webb T., Butler J.V., Clarke D.J. \& Boer H. (2001) Population prevalence and estimated birth incidence and mortality rate for people with Prader-Willi Syndrome in one UK Health Region. Joumal of Medical Genetics 38, 792-8. 


\section{Abstract}

The identification of al] people in one Health Region, who may have the genetically determined Prader-Willi syndrome, was attempted by direct contact with health, social, and educational services and through the PWS Association and by appeals through the local media.

From a total of 167 people referred with possible PWS, 96 were included having PWS on genetic and/or clinical grounds. Variation across the eight counties in the Health Region and observed gender differences enabled us to estimate the numbers of people with PWS which were missed by the ascertainment methods used. From the age related prevalence data, both birth incidence and mortality rate were estimated.

We concluded that a lower bound for the population prevalence was $1: 52000$. A lower bound for the birth incidence was estimated to be 1:29 000. The mean mortality rate was estimated to be $3 \%$ per year across the age range or about $7 \%$ per year above age 30 , depending on assumptions made.

While differential ascertainment across the age range might be a factor, we conclude that these findings largely indicate significant morbidity and associated mortality. We predict that this is because of a falure to manage weight gain in later life and thereby prevent associated obesity related health problems

Prader-Willi syndrome (PWS) is a genetically determined disorder in which the absence of expression of one or more maternally imprinted gene(s) in the chromosomal region 15q11-13 results in a characteristic facial appearance, learning disabilities (mental retardation), and severe overeating behaviour owing to an abnomal satiety response to food intake, together with a range of other behaviours. Initially, as reported by Prader et al (1956) PWS was conceived as a syndrome of obesity, short growth, cryptorchidism, and mental retardation following hypotonia in the neonatal period. As more and more people with PWS were reported and research into the syndrome began, behavioural characteristics and other clinical features were added, culminating in the consensus diagnostic criteria (Holm et al, 1993). Concurrently, the genetics of the disorder were receiving attention. First was the discovery that for many there was a visible chromosomal deletion in the proxinal part of the long arm of chromosome $15(15 \mathrm{q}$ 11- 13). Reports of an apparently similar deletion being associated with a phenotypically very different syndrome (Angelman syndrome, AS) (Magenis et al, 1987). and the observation that PWS was the result of a deletion on the chromosome 15 of paternal origin, and AS the chromosome 15 of matemal origin, led to the recognition that gender specific imprinting of genes at that locus accounted for two diverse syndromes being associated with apparenty similar chromosomal deletions (Nicholls et a, 1989). Maternal chromosome 15 disomies, mutations of 
an imprinting centre, and chromosomal translocations accounted for non-deletion cases of PWS (Nicholls, 1993).

In published reports on Prader-Willi syndrome (PWS), prevalence has been variously quoted as "about 1 in 25000 live births" (Butler 1990), "between one in 25 000 and one in 10000 live born children" (Akefeldt et al, 1991), "[estimates] wary 6-fold from 1 in 5000 to $10000 ; 1$ in 10000; 1 in 15000; 1 in 25000; to 1 in 10000 to $30000^{\prime 3}$.8 Only two estimates appear to be based on epidemiological data, those of Akefeldt ef al and Burd ef al (1990). In the latter North Dakota study, the authors surveyed paediatricians, neurologists, and clinical geneticists and also contacted the state's comprehensive evaluation centre, the state hospital, the state institution for the "mentally retarded", and group homes for the developmentally disabled. including one for people with PWS. In most communities, at least four of these sources of information were consulted. Each was sent a one page questionnaire pictorially illustrating the signs of PWS to aid identification. The response rate was 99\%. These procedures yielded eight males, eight females, and one person whose gender was not given, with an age range from 9 to 30 years. At that time the population of North Dakota for that age range was 263444 , giving a prevalence rate of $1: 16062$, equivallent to $1: 38395$ in the entire population. No figures were given for the number of cases with a genetic diagnosis.

In the study of Akkefeldt ef al (1991), the authors estimated the prevalence of PWS in the age range 0 to 25 years in the rural Swedish county of Skaraborg, by surveying paediatricians, neuropaediatricians, child psychiatrists, school heath visitors, general practitioners, and doctors working in the fields of general medicine, rehabilitation, and mental disabilities. The authors circulated diagnostic criteria for PWS to these professionals and also invited all school nurses in the county to seminars where PWS was described. Requests were made that all people with possible PWS be notified to them. All people with possible PWS were cxamined by a neuropaediatrician, a child psychiatrist, a child psychologist, and a speech pathologist. Clinical diagnoses were made on the basis of their findings. Eleven people (seven male and four female) were considered definitely to have PWS and a further five (two male and three female) were considered probable. These numbers gave a population prevalence of "clear PW/S" up to the age of 25 years of $1: 8500$ and between 7 to 25 years 1:8000. If the people with suspected PWS were also included, these prevalence rates became 1:6700 and 1:5000, respectively.

These different population estimates can be reconciled in several ways, including sampling errors, differences in ascertainment, high mortality rates between the ages of 25 and 30 , or varying prevalence rates in different populations. The first two of these probably apply, at least to some extent. High mortality rates have never been reported as specific to a particular age group. The final explanation is at odds with the accepted view of PWS as a randomly occurring de novo genetic mutation. This accepted view is supported by the fact that in both studies the 
gender ratio is close to $1: 1$, if, in the Swedish study, people with "probable" as well as "definite" PWS are counted. Clearly, if the explanation was that prevalence rates vary depending on the population studied, then these estimates cannot be generalised to other countries.

We describe a population prevalence study in the UK that was undertaken as part of a larger study investigating the heterogeneity of the PWS phenotype. The aim was to locate all definite or possible cases of PWS in the previous Anglia \& Oxford Health Region, comprising the counties Bedfordshre, Berkshire, Buckinghamshire, Cambridgeshire, Norolk, Northamptonshire, Oxfordshire, and Suffolk (population approximately 5 million people). All ages were to be included, so as to obtain some estimate of mortalicy rates in the PWS population, and as far as possible the diagnosis was confimed by the routine diagnostic method using SNRPN medzylation (Glenn et al 1996), or, if that was not possible, the presence of accepted diagnostic criteria.

\section{Methods}

Since the ideal method of random sampling is impractical for rare disorders requiring clinical diagnosis, the method of counting all known cases in the region was used, as it was in the studies quoted above. The size of the region studied was chosen after administrative and statistical considerations. The region chosen comprises approximately five million people, about one tenth of the population of England and Walles. Using a Health Region is advantageous administratively, since ethics committees and laboratory and clinical facilities are region based. The population prevalence rates found in the previous studies quoted above, together with statistical power considerations, suggested that the study of the phenotype would require a population of at least this size.

Heath, education, and social services professionals (as detailed in the appendix) were contacted and sent a list of common PW/S characteristics and asked if they knew of anyone fulfilling at least half of these (see table 4). In addition, the UK PWS Association was asked to advise about numbers known to the Association living in the study area. To preserve anonymity, initials, gender, and date of birth (or age) of their nominees were requested; these were necessary in order to avoid double counting of people who possibly had the syndrome. They were also asked if they were prepared to forward a letter from us to the person with PWS inviting their participation in the phenotypic sudy. (Note that anonymity and indirect contact were conditions of ethical approval for the study; therefore no other details are known of people nominated who did not contact us). All responders who were willing were recruited into the phenotypic study for cognitive assessment of the person with PWS and in depth interviews (including a section asking 


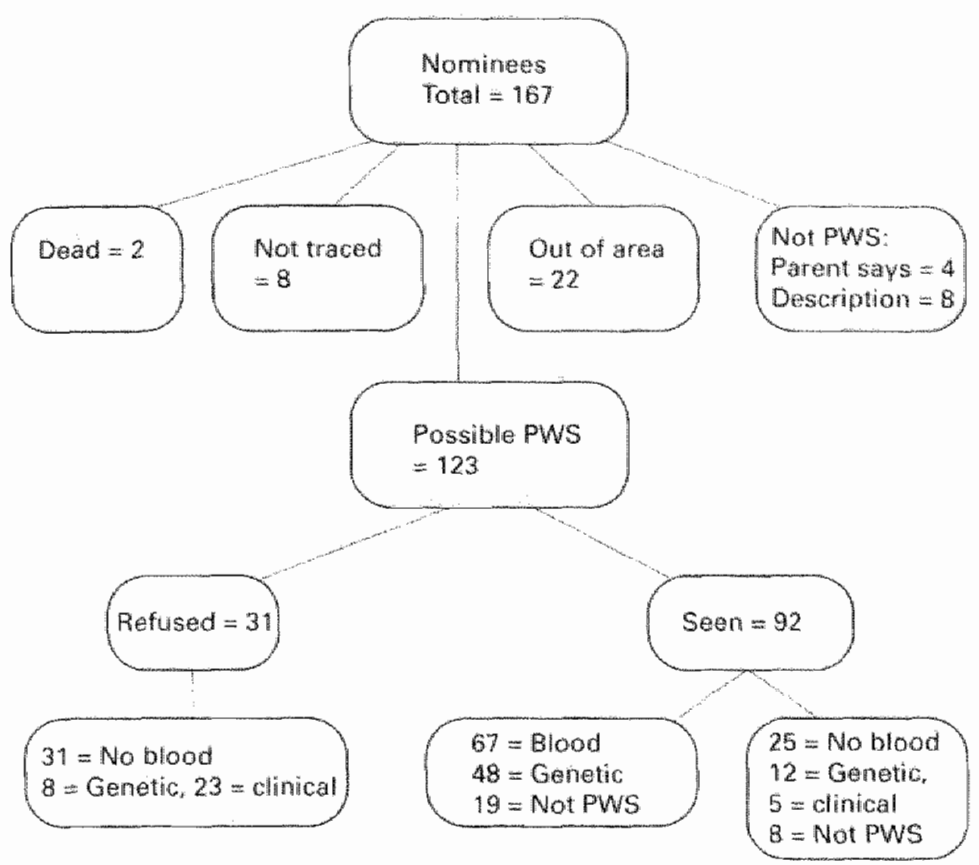

Figure 1. Breakdown of group.

about the presence or not of the symptoms included in the consensus diagnostic criteria) with a parent or main carer or both. Blood samples were later collected from all those who were willing and able to provide samples. In some cases, when the subject was unwilling or there were difficulties obtaining a sample, permission was given for access to previous genetic records. For most participants in the phenotypic sudy, therefore, a diagnosis of PWS could be confirmed either clinically (parents/carers were asked at interview about the presence or not of consensus diagnostic criteria) and/or genetically. In the region, there is a collection of group homes for people with PWS. Only those funded from within the study region were included in the population part of the study, the others coning from outside the region.

During the active period of the study, between September 1998 and June 2000 , 167 people with a diagnosis of PWS or with PWS symptoms were notified to us from the sources listed earlier, including nominations by parents and other relatives of people with eating problems and other PWS symptoms who responded to media appeals. Fig 1 shows the breakdown of this group in terms of participation in the phenotypic study, blood samples obtained, genetic test results, and clinical diagnoses. This figure shows that after eliminating those nominees who were found to have died, were untraceable, found on preliminary investigation not to have PWS, or had moved out of the region, 123 people with possible PWS were 
Table 1. Population classification of nominees by source

\begin{tabular}{llllll}
\hline & Genet PWS & Clinic PWS & Not seen & Non-PWS & $\begin{array}{l}\% \text { positive of } \\
\text { those seen }\end{array}$ \\
\hline Genet Lab & 32 & 0 & 8 & 0 & 100 \\
Doctors & 87 & 5 & 23 & 15 & 86 \\
PWSA & 36 & 1 & 13 & 10 & 79 \\
Relative & 6 & 0 & 1 & 13 & 32 \\
Head teacher & 8 & 0 & 2 & 0 & 100 \\
Social Services & 10 & 1 & 3 & 2 & 85 \\
Home/centre manager & 1 & 0 & 0 & 1 & 50 \\
\hline
\end{tabular}

included. Of these 123,31 with a previous diagnosis of PWS did not wish to participate in the phenotypic study, and therefore the only data are those provided by those who referred the person to the research study. Eight of these were from genetic laboratories, and in the remaining 23 cases the genetic status was not given. Many (75) of the 123 nominees were from multiple sources, as detailed in table 1; many were nominated as "maybe", particularly parental nominations. None of the 31 not seen were "maybe". Table 1 also gives the hit rates (that is, number of positive cases) for the cases seen among the various sources.

Among the 92 who agreed in principle to participate in the phenotypic study, genetic testing was possible for 67 participants. Of the other 25 , eight were considered not to have PWS at interview. With 12, results of previous genetic tests were obtained confirming the diagnosis. The remaining five were included as they met clinical criteria. Altogether, 27 people out of 92 were deemed not to have PWS (the above eight on clinical grounds only, 11 on both clinical and genetic grounds, a further eight on genetic grounds) and 65 met clinical and/or genetic criteria. Our report is based on the latter group ( 65 people) and the 31 who did not wish to be involved in the larger study, giving a total of 96 .

Fig 2 shows the frequencies by age (standardised to midnight 31.12 .99 ) of these 96 people.

\section{Results}

There are two obvious potential sources of error in our results, false positives and missing cases. With respect to the former, among those people seen, we have 60 with genetic confirmation and five who did not have a genetic test (three refused, two tried and failed) but met clinical diagnostic criteria. Among the 31 not seen, eight had genetic confimation; table 1 suggests that at least $80 \%$ of the others are expected to be positive, so that we should expect at most five false positives even before consideration of the firmness of the nominations, which led us to include all cases. 
Figure 2. Frequencies by age.
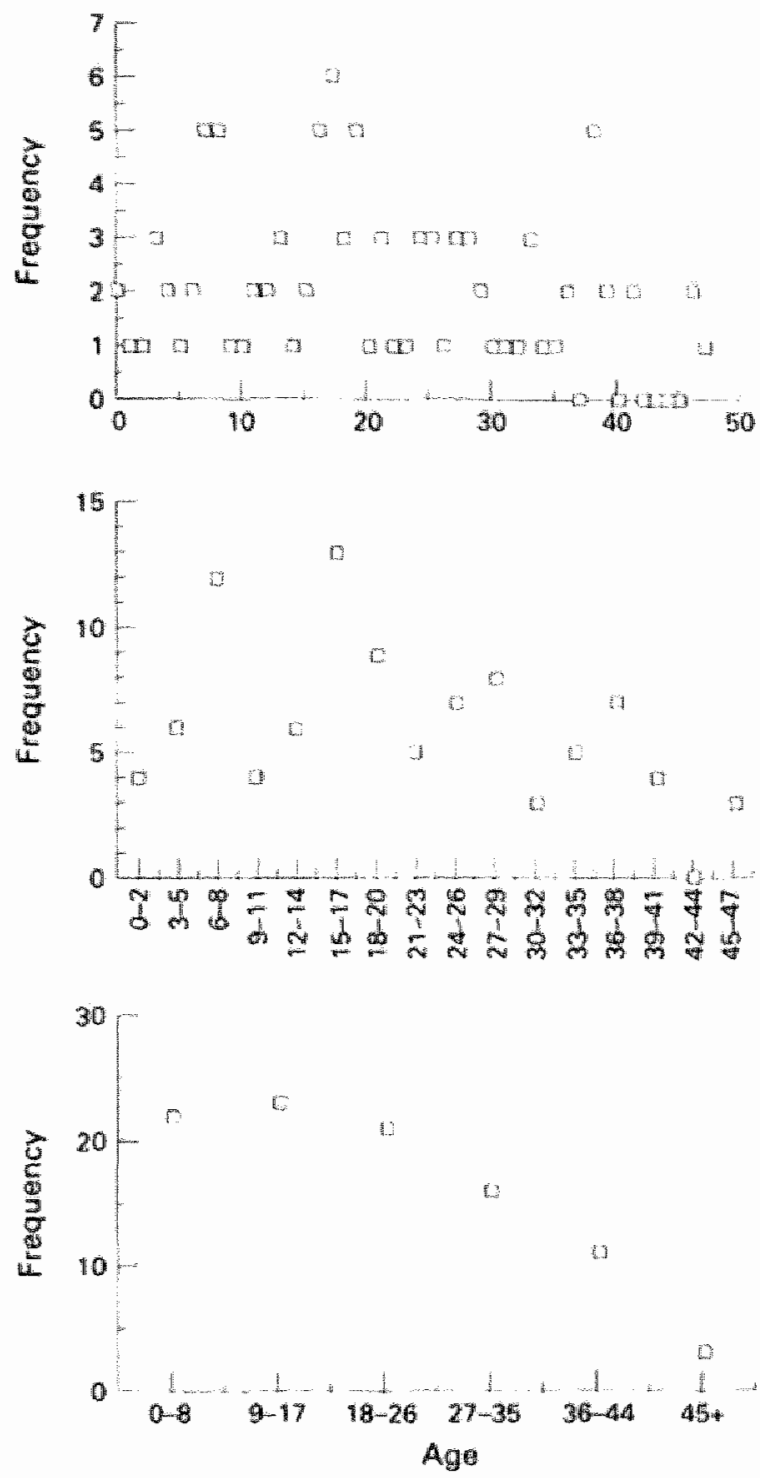

With respect to missing cases, we believe this to be more of a problem for several reasons. The weakest evidence for missing cases is regional variations in prevalence, much stronger are age by county and gender variations.

Table 2 gives the numbers of people with PWS divided according to each county. The overall population rate was 1:52000, with county rates varying from 1:42 000 to $1: 67000$ (not statistically significant). This could indjcate that identification was better in some areas than in others, and a conservative estimate suggests that there 
Table 2. Mumbers, gender differences and ages of people with PWS identified by county

\begin{tabular}{|c|c|c|c|c|c|c|c|c|}
\hline & Beds & Berks & Bucks & Camb & Norf & Worth & Oxan & Suff \\
\hline Male & 7 & 4 & 7 & 10 & 9 & 6 & 5 & 7 \\
\hline Female & 4 & 6 & 6 & 3 & 3 & 2 & 4 & 5 \\
\hline Not stated & 1 & 2 & 1 & 1 & 3 & & & \\
\hline Totals & 11 & 11 & 15 & 14 & 13 & 11 & 9 & 12 \\
\hline \multicolumn{9}{|l|}{ Age (years) } \\
\hline $0-2$ & - & 2 & $\mathbb{1}$ & - & - & - & - & 1 \\
\hline $3-5$ & - & - & 2 & 3 & 1 & - & - & - \\
\hline $6-8$ & 2 & 4 & 2 & 3 & - & - & 1 & - \\
\hline $9-11$ & - & 1 & 1 & 1 & - & - & - & 1 \\
\hline $12 \cdot 14$ & 1 & 1 & 2 & 1 & - & - & 1 & - \\
\hline $15-17$ & - & 2 & 2 & 3 & - & 2 & 2 & 2 \\
\hline $18-20$ & 1 & - & 1 & 1 & 3 & $\mathbb{1}$ & - & 2 \\
\hline $21-23$ & 1 & - & - & - & 3 & - & - & 1 \\
\hline $24-26$ & 2 & - & 1 & 一 & - & 1 & 3 & - \\
\hline 27.29 & 1 & - & 1 & 1 & 1 & 3 & - & 1 \\
\hline $30-32$ & - & - & 1 & - & 1 & - & 1 & - \\
\hline $33-35$ & $\mathbb{1}$ & - & - & - & - & 2 & - & 2 \\
\hline $36-38$ & 1 & 1 & 1 & 1 & 1 & - & $\mathbb{1}$ & 1 \\
\hline $39-41$ & - & - & - & - & 3 & 1 & - & - \\
\hline $42-44$ & - & - & - & - & - & - & - & - \\
\hline $45-47$ & 1 & - & - & - & - & 1 & - & 1 \\
\hline
\end{tabular}

may be another seven people with PWS within those counties with low reported rates. But such variation may also indicate random variation and that the two previous prevalence study estimates can be reconciled by random sampling. North Dakota is similar in population size to a single county, while Skaraborg is only half that popullation size.

Ages were standardised to midnight on 31 December 1999. Table 3 gives the numbers of people with probable or definite PWS across the age range. Assuming that roughly the same number of babies with PWS are born every year, an examination of the overall age structure of our sample suggests that some very young children with PWS may not yet have been diagnosed (table 3), awaiting the onset of eating and behavioural problems to prompt investigations. (This is supported by age at diagnosis data collected in interviews; new diagnoses were common in the first year and between 3 and 5 years). If we assume a zero mortality rate for children under 6 , table 3 suggests that as many as five children with PWS may not yet have been diagnosed. The age structure of identifed cases within counties also supports the "missing cases" hypothesis. In Norfolk, for example, only one person under 18 years out of 13 was identified, while in Berkshire there was only one person with PWS over 17 years out of 11 identified.

In all but one county, we found more males than females $(55$ males, 33 females, eight gender not stated). Assuming that the gender ratio in PWS is close to unity, as 
Table 3. Numbers across the Region divided by age and sex

\begin{tabular}{lllll}
\hline Age (years) & Malle & Female & Not stated & Total \\
\hline $0-2$ & 3 & 1 & & 4 \\
$3-5$ & 3 & 2 & 1 & 6 \\
$6-8$ & 5 & 6 & 1 & 12 \\
$9-11$ & 3 & 1 & & 4 \\
$12-14$ & 3 & 3 & 1 & 6 \\
$15-17$ & 6 & 6 & & 13 \\
$18-20$ & 7 & 2 & & 9 \\
$21-23$ & 4 & 1 & & 5 \\
$24-26$ & 6 & 1 & 1 & 7 \\
$27-29$ & 6 & 1 & 1 & 8 \\
$30-32$ & 1 & 3 & 1 & 3 \\
$33-35$ & 1 & 3 & 1 & 5 \\
$36-38$ & 3 & 0 & 1 & 7 \\
$39-41$ & 3 & 0 & & 4 \\
$42-44$ & 0 & 2 & & 0 \\
$45-47$ & 1 & 33 & & 3 \\
Total & 55 & & & 96 \\
\hline
\end{tabular}

the two previous studies found, it therefore seems that females with PWS were either specifically not being diagnosed or were less likely to be referred to the study than males, and we estimate there may be between 14 and 30 more females with PWS in the region. Of the four infants aged $0-2$, three were male, and in botl Cambridgeshire and Norfolk we found six more males than females (table 3). Unlike the previous population studies which yielded 17 probable cases, age range 9-30, in North Dakota and 16 cases, age range 0-25, in Skaraborg, we have a sufficiently large sample to look at age frequencies. A number of different methods are available to estimate birth incidence from the raw data (which is shown at the top of fig 2). All methods depend on the different assumptions we are prepared to make, the chief common assumption being that PWS results from a randondly occurring genetic fault, that is, the age frequencies constitute a random sequence about a steady incidence rate. (We note that this sequence would vary slightlly if we chose a different date for age standardisation.) This sequence can be smoothed in various ways, as shown in fig 2 , where the data have been grouped in three-year intervals and in nine-year intervals. In the latter case, the data were fit by a quadratic with an $\mathrm{R}^{2}=0.996$ (an exceptionally good fit). We have also assumed no false positives in ascertainment. Assuming no missing data would then give lower bounds for the prevalence and birth incidence for a particular method of calculation of the latter.

Assuming no missing data and using the smoothed data obtained from nine year groupings, we find rates of $22,23,21$ cases in the first three nine year periods, a rate of $1: 28000$, with a zero mortality rate to age 27 and thereafter an average rate of $6.1 \%$ (from 2.44 to 0 in 20 years). 


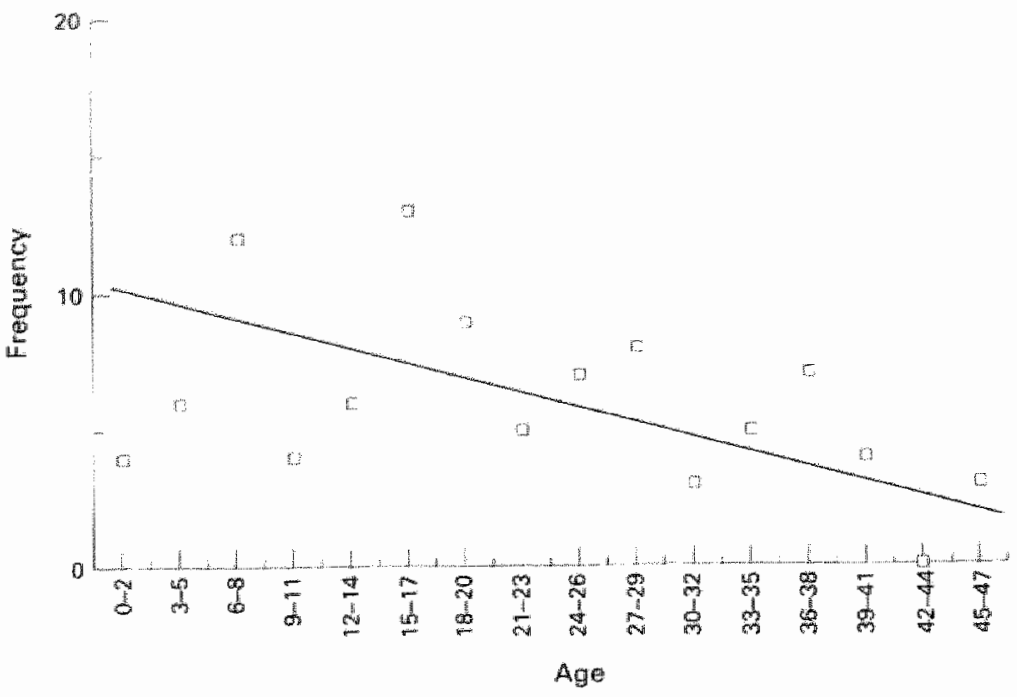

Figure 3. Birth incidence and mortality rate.

Table 4. Information given to contacts

Do you know anyone who has been diagnased as having PWS or may have had five of the following characteristics?

Floppiness at birth.

Initial failure to thrive or difficulty in sucking.

The development of severe overeating and rapid weight gain in early childhood.

Obesity or the need for weight control.

Problems with sexual development (for example, undescended testes, delayed periods).

Some leaming disability (mental handicap).

Small hands and feet.

Short stature or the need for growth hormone.

An abnormality of chromosome 15 .

A similar calculation with eight year grouping (Git by a quadratic with $\mathrm{RZ}=0.85$ ) and rates of $17,17,25,17$ cases in the first four eight year periods, gives a rate of $1: 29000$, with a zero rate of mortality to age 32 and thereafter an average rate of $7.9 \%$ (from 2.375 to 0 in 15 years).

The paragraphs above suggest that data are missing (females) but may be fairly evenly spread across the age groups (age by county). If this is the case, then the falling number of cases with age is probably because of mortality and we can use 
the whole distribution to estimate lower bounds for birth incidence and mortality rate. Fig 3 illustrates this method, where the fitted line is the result of ignoring ages $0-5$ (since we hypothesised that cases might be missing in this age range, their eventual diagnosis awaiting the onset of the second phase of PWS symptoms). A straight line, as shown, was found to be near the optimum fit with $R^{2}=0.41\left(R^{2}\right.$ could be increased to 0.44 by fitting a fourth order polynomial). This would imply a steady death rate across the age groups, rather than an increasing rate with age. (Anecdotally, we were told of two people with PWS who died before we could see them, a 3-4 year old girl and a 13-year-old boy). From fig 3 , we see that 10.2 children are expected in the age range $0-2$ (that is, in three years), giving a birth incidence of 1:20 000 in the population studied (68 000 births per year). We also see that the overall death rate in this PWS population is about $3 \%$ per year (that is, from 3.4 to 0 over 56 years). This compares with an overall death rate in the population of England and Wales of about $1 \%$ per year, and only about $0.13 \%$ per year in those up to 55 years.

Another set of assumptions is that advances in genetics would result in increasing numbers of accurate diagnoses, so that ascertainment by age would be a step function, with steps corresponding to identification of deletions (c 1981, age 18), maternal disomy ( 1989 , age 10), and the imprinting centre methylation tests ( $c$ 1995, age 4). This is clearly not supported by our data.

A final set of assumptions is that data are missing only, or mainly, from the older age groups. This is consistent with the 8 year and 9 year groupings if we assume little or no mortality. But there are two arguments against this. First, the county data and age at diagnosis data suggest that missing cases are not confined to older age groups; in some counties such age groups are over-represented. Secondly, several older people were nominated as possible PWS and were interviewed but were found to be genetically negative, so failure to find older people was not because of lack of candidates.

\section{Discussion}

There are a number of problems related to this method of conducting a prevalence study of PWS. The three main questions are: how should PWS be defined?, do all nominees have PWS?, and do all other people in the region not have PWS? The first question still remains to some extent unresolved as neither the genetic test nor the clinical diagnosis is $100 \%$ definitive. Although it is generally agreed that genetic abnormalities of the Prader-Willi critical region on the chromosome 15 of paternal origin underlie the clinical manifestations of the syndrome, and that the dual genetic tests used will detect probably $99 \%$ of such abnormalities, the precise genes) involved have not been identified and therefore a definitive genetic test is 
not available. From the clinical perspective, it is clear that there are other chromosomal abnomalities, such as deletions of part of chromosome 6 leading to PWS-like symptoms (one such case is known to us), which show that reliance on clinical diagnosis may result in the inclusion of those whose clinical phenotype results from a different genotype. There was one person in our population study with a score of 9 on the diagnostic criteria who was excluded when extensive genetic tests proved negative.

With respect to the second question, we cannot be certain whether everyone included in this study definitely has PWS. Some nominated did not respond to requests to take part in the phenotype study, and therefore we have had to rely on previous clinical and/or genetic data. Others did not wish for a blood test and, in the absence of a previous test, a genetic diagnosis was not possible. However, we have been restrictive about who was or was not included. We excluded all those 27 nominees who did not meet either clinical criteria (no blood), genetic criteria, or both clinical and genetic criteria for PWS. These people had nomal matemal and paternal methylation patterns and gene expression at the SNRPN locus (where blood was available) and typically had less than $50 \%$ of the required diagnostic criteria. We have included 60 people who had definite genetic diagnoses and five others who had definite clinical diagnoses (in the absence of genetic confirmation). The 31 who did not wish to participate in the phenotypic study were all included as having PWS, as detailed earlier.

Our assessment of findings relevant to the third question leads us to believe that there are other people in the region who do have PWS. If it is assumed that the syndrome is indeed caused solely by random genetic error (that is, with no environmental component), it follows that prevalence should not be biased with respect to gender, race, or environment. Although some relationship to paternal occupation in the hydrocarbon industry has been described in the past, which might possibly lead to clustering (A kefeldt et al, 1995), this has not been replicated. With the above assumptions, it is possible to look for internal consistencies within our regional sample, such as by county, by gender, or by race. There is not enough variation in racial background to test our sample with respect to race. In all but one county, we found more males than females ( 55 males, 33 females, eight gender not stated); this agrees with previously published reports (Cassidy ef al, 1997). Assuming that the gender ratio in PWS is close to unity, as the two previous studies found, it therefore seems that most of any missing cases (estimate 14 to 30 ) will be female. This is not surprising; at all ages, suspicion of PWS is more likely in males (hypogonadism in childhood, short stature with obesity in adulthood). However, in two of the three age groups in which females did in fact outnumber males, the age group was in the over 30s raising the possibility that, with PWS, as in the general population, women outlive men. Given the assumptions about those with PWS not identified, the findings suggest that a population prevalence of 1.52000 
is a lower bound and that the true prevalence is somewhat higher, nearer to $1: 45,000$.

Unlike the two previous population surveys of PWS the present survey was large enough to allow the age structure of the PWS population to be examined. We were able to estimate both birth incidence and death rate. The estimate of mortality rate assumes that there is no systematic age related ascertainment bias. For example, the age data might have been stepped corresponding to advances in awareness and diagnostic refinements, but the age structure found argues against such a cohort effect. However, it is possible that there are older adults with PWS born before the syndrome was well known and in whom the diagnosis has never been considered. While this possibility cannot be ruled out, we would argue that an increased mortality rate is the most likely explanation of the age profile of this population based cohort of people with PWS. High rates of both physical and psychiatric disorder have been reported (Greenswag, 1987) and preliminary analysis of further data from this study provides evidence of significant morbidity. It would seem likely that obesity and associated complications are the main contributory factor. However, a systematic prospective study is required to establish whether this is the case or not. The physical health needs of people with learning disability are often not properly assessed or adequately met (Lennox and Kerr, 2000 ), and disorders such as type 2 diabetes might easily be missed in this population. There is also concern that misguided support strategies in adult life may result in marked weight increase and that resultant obesity related problems then occur.(Dykens et al, 1997; Holland and Wong, 1999).

JW and JB were supported by a grant from the Wellcome Trust with further support from the PWS Association. We are very grateful to both organisations for their funding of this study. We would specifically like to thank Rosemary Johnson of the PWSA for all her administrative support, and all those people with PWS, their families, and other carers and many people who helped us with this study. This includes medical professionals, those working in genetic clinics, social services placements, education and learning disability services, and the media for contacting peoplle on our behalf.

\section{References}

Akefeldt A., Anvret M., Grandell U., Nordlinder R. \& Gillberg C. (1995) Parental cxposture to hydrocarbons in Prader-Willi syndrome. Developwental Medicine and Child Nerology $37,1101-9$.

Akefeldt A., Gillberg C. \& Larsson C. (1991) Prader-Willi syndrome in a Swedish rural county: Epidemiological aspects. Develophenthal Medicine and Child Neurology 33, 715-21. 
Burd L. Vesely B., Martsolf J. \& Kerbeshian J. (1990) Prevalence study of Prader-Willi Syndrome in North-Dakota. Anerican Jonratal of Medical Gervetics 37, 97-9.

Butler M.G. (1990) Prader-Willi syndrome: current understanding of cause and diagnosis. Anerican Jouthul of Medical Genetio 35, 319-332.

Cassidy SB, Forsythe M, Heeger S, Nicholls RD, Schork N, Benn P, Schwartz S. (1997) Awerican Joumal of Medical Generies 68, 433-40.

Dykens E.M., Gof B.J.,Hodapp R.M., Davis L., Devanzo P. Moss F., Halliday J., shah B., State M. \& King B. (1997) Eating themselves to death: have personal rights" gone too far in treating people with Prader-Willi syndrone? Mental Retardation 35, 312-4.

Glenn C.C., Saitoh S., Jong M.T.C., Filbrandt M.M., Surti U., Driscoll D.J. \& Nicholls R.D. (1996) Gene structure, DNA methylation, and imprinted expression of the human SNRPN gene. American Jonmal of Human Gewetics $58,335-46$.

Greenswag L.R. (1987) Adults with Prader-Willi syndrome: a survey of 232 cases. Depelopmental Medicite and Child Nemrology $29,637-48$.

Holland A J. \& WongJ. (1999) Genetically-determined obesity in Prader-Willisyndrome: the ethics and legality of treatment, foumal of Medical Ethics $25,230-6$.

Holm V.A., Cassidy S.B., Butler M.G., Hanchett J.M., Greenswag L.R., Whiman B.Y. \& Greenberg F. (1993) Prader-Willi syndrome. consensus diagnostic criteria. Pediatrics 91 , 398-402.

Lemnox N.G. \& Ker M.P. (2000) Primary health care and people with an intellectual disability: the evidence base. Journal of Intellectual Disability Researd 41, 365-72

Magenis R.E., Brown M.G., Lacy D.A., Budden S. \& LaFranchi S. (1987) Is Angelman syndrome an alternate result of del $(15)(\mathrm{g} 11 \mathrm{q} 13)$ ? American Joumal of Medical Genetics 28 , 829-38.

Nicholls R.D. (1993) Genomic imprinting and candidate genes in Prader-Willi and Angelman syndromes and mouse models. Current Opiwion in Genetios \& Developmen 3, 445-56.

Nicholls R.D., Knoll.J.H., Butler M.G., Karam S. \& Lalande M. (1989) Genetic imprinting suggested by matemal heterodisomy in nondeletion Prader-Willi syndrome. Nature 342 , $281-5$.

Prader A., Labhant A. \& Willi H. (1956) Ein Syndrom von Adipositas, Kleinwuchs, Kryptorchismus und Oligophrenie nach myatonieartigem Zustand im Neugeborenalter. Silutizerische Medizinishe Wodhenschrift 86, 1260-1. 


\section{Appendix}

\section{Contacts with groups and professionals in search for people with PWS}

- PWSA (UK)

- Genetics Departments - all known postholders.

- Paediatricians - registered members of the Royal College of Paediatricians.

- Paediatricians - listed in the Anglia \& Oxford Health Region Handbook.

- Psychiatrists in learning disabilities - registered members of the Royal College of Psychiatrists.

- Psychiatrists in learning disabilities - listed in the Anglia E Oxford Health Region Handbook.

- GPs - Cambridge area only (not cost effective).

- Dieticians.

- Community nurses

- County Councils

- Special education.

- Social services.

- Special schools for learning disabilities.

- Residential homes for learning disabilities.

- Media - newspapers, television, radio. 

Chapter 3 a

Cognitive abilities and genotype in a population-based sample of people with Prader-Willi syndrome

Published as:

Whittington J., Holland A., Webb T., Butler J., Clarke D. \& Boer H. (2004) Cognitive abilities and genotype in a population-based sample of people with Prader-Willi syndrone. Joumal of Intellectual Disability Research 48, 172-87. 


\section{Summary}

Background. Prader-Willi Syndrome (PWS) is characterised by extreme floppiness at birth, impaired sexual development, short stature, severe over-eating, characteristic physical features, and learning disabilities. Impaired social cognition, literal mindedness and cognitive inflexibility are also present. The syndrome has two main genetic sub-types that both result in the failure of expression of maternally imprinted genes on chromosome 15 at the locus $\mathrm{q} 11-13$

Methods. Through multiple sources we attempted to identify all people with PWS living in one Health Region in the UK. Additional people with PWS identified in other regions were also recruited to augment the study sample. A comparison group of people with learning disabilities due to aetiologies other than PWS was also identified. All people from these three groups, over age three, who gave their consent, were assessed using tests of ability and attainment. In addition, their main carers were interviewed using a semi-structured interview. Blood samples for genetic diagnosis were obtained from all consenting participants.

Findings. The IQ distribution of the population sample was approximately normal with an mean IQ 40 points below that of the general population. There were systematic differences between the two main genetic subtypes. Those with disomies differed in cognitive profilles from both those with deletions and the comparison LD group (the latter two groups were very similar) in terms of better verbal abilities and impaired coding ability. Some people with PWS deletions had strong visuo-spatial skills.

Interpretation. We propose that the normal distribution of $1 Q$, shifted downwards relative to that of the general population, is the result of a global effect on IQ of the PWS gene(s), and that the different cognirive profile seen in those with chromosome 15 maternal disomies is a specific effect of a gene, or genes, on chromosome 15 which is differentially either expressed or not expressed in those with disomies relative to those with deletions. One hypothesis is that these subtle cognitive differences are a manifestation of the genetic influences of gender specific imprinted genes on cerebral lateralisation. This requires further investigation.

\section{Introduction}

For those genetic syndromes associated with early developmental delay and learning disabilities (LD) we assume that the gene(s) associated with a particular syndrome is (are) normally expressed in the developing brain, and that failure of expression leads to abnomal brain development, and thus impaired cognitive, emotional and/or social development. Currently, the particular developmental 
abnormalities leading to the learning disabilities, the patterns of general ability, and the factors that influence the extent of variation in the degree of severity associated with different syndromes are often unknown. In the non-learning disabled population general ability is influenced both by genetic and environmental factors with the genetic component estimated as accounting for between 0.50 and 0.80 of the variance, and being mediated by many genes (Falconer, 1981).

The study of genetic syndromes associated with learning disabilities can give information about the genetic component of cognition. Potentially important pointers to the nature of the genetic influences on cognition include: the distribution and heritability of general ability in a given syndrome, the systematic differences in profiles of abilities between syndromes, and a specific ability or disability associated with a syndrome. Ideally, such investigations require representative samples (or repeated random sampling) of those with the syndrome. For many genetic syndromes such population-based cohorts have not been obtained.

Prader-Willi syndrome (PWS) is a genetically determined disorder in which the absence of expression of one or more maternally imprinted gene(s) at the chromosomal locus $15 q 11-13$ results in extreme floppiness at birth, a characteristic physical phenotype, and the presence of mild to moderate learning disabilities (Cassidy, 1997). Severe over-eating behaviour, due to an abnormal satiety response to food intake, is a characteristic behaviour (Holland et al. 1993), as are obsessive behaviours, and temper outbursts (Clarke et al. 2002; Holland et al. 2003). As more people with the syndrome were reported and research into the syndrome developed, Consensus Diagnostic Criteria for the clinical diagnosis were devised (Holm et al. 1993). Concurrently, the genetics of the disorder were receiving attention leading to the observations of a chromosomal 15 deletion (Ledbetter et al. 1981), and later of other genetic abnomalities (matemal chromosome 15 uniparental hetero- or isodisomies, unbalanced translocations, and imprinting centre mutations) of this region of chromosome 15 (Nicholls, 1993), all apparently associated with a similar PWS phenotype. All those with PWS are now thought to have a genetic abnormality the result of which is the failure of expression of specific genes located at the 'PWS region' of chromosome 1.5 $(15 \mathrm{q} 11-13)$. The significance of both the chromosome 15 deletion (found in approximately $70 \%$ of people with PWS), the chromosome 15 maternal uniparental disomy (found in about $25 \%$ of people with PWS), and the very rare imprinting centre mutations lies in the fact that some of the grenes in the PWS critical chromosomal region are matemally imprinted; that is, these genes are expressed only when inherited via the father, the genes on the maternal chromow some being normally silenced (imprinted). Although both main genetic subtypes have the similar characteristics of PWS, there are differences in phenotype between these two subtypes that are likely to be a reflection of specific genotypic differences between deletions and disomy (Mitchell et al. 1996). Most strikingly 
those with disomy have a very high risk of developing a psychotic illness in adult life compared to those with deletions (Boer et al. 2002).

The literature on cognitive functioning and achievement in PW/S is somewhat heterogeneous. People with PWS have been variously deseribed as having "usually mild to moderate mental retardation", "normal intelligence in a significant number of cases" "leaming disabilities", "characteristic cognitive weaknesses" "good reading skills", and "weakness in arithmetic skills". It is not always possible to deduce from the text on what basis these claims have been made. Generally, the first two are based on the results of an $1 Q$ test indicating that $I Q$ is wisually found to be in the ranges $50-70$ or $50-85$ (Curfs et al. 1991; Curfs and Fryns, 1992), and as many as one quarter (or one fifth) have an $1 Q$ over 70 (or over 80, depending on the rating systen used) (Curfs et al. 1991; Curfs \& Fryns, 1992). The other descriptions are usually based on discrepancies between IQ subscale scores or on attaiment tests (Holm, 1981; Dykens et al. 1992). In the UK, the term "leaming disabilities" (equivalent to the term "mental retardation") refers to a global lowering of ability scores in the context of early developmental delay, whereas "specific learning disabilities" refers to conditions such as dyslexia and dyscalculia in which specific areas of ability are affected, resulting in particular areas of attaimment being abnomally low in relation to general levels of IQ. Thus, the evidence indicates that in PWS there is both a global lowering of ability, and possibly more specific cognitive disabilities.

Reports of cognitive functioning in PWS have suffered from one or both of two methodological drawbacks. First, they have been based on volunteer of clinical samples of people with PWS and no attempt has been made to assess the representativeness of such samples. Secondly, early reports in particular (some based on clinical criteria and some on visible deletions only) may have misclassified individuals as having PWS when in fact they did not. Experience from our present study would suggest that prior to the establishment of genetic criteria a proportion of all 'cases' were misclassified (Whittington et al. 2001). These drawbacks may explain the discrepancies and contradictions within the literature. However, some findings have been replicated in those with genetic confirmation of PWS, and a meta-analysis of 56 studies has been published (Curfs and Fryns, 1992). It is now generally accepted that, as a group, people with PWS perform better on visuo-spatial tasks, have poor short-term memories, deficits in sequential processing, poor socialisation skills and exceptional skills with jigsaw puzzles (Warren \& Hunt, 1981; Dykens ef al. 1992; Cassidy ot al. 1997). It has also been reported that $I Q$ is not related to weight and does not decline with age (Dykens et a. 1992).

Since the two main categories of genetic abnomality in PWS were established, comparisons of cognitive performance between deletion and disomy subgroups have been described. Roofet al. (2000) found that people with disomies had a 
higher average verbal intelligence (VIQ), whereas there was no significant difference in average performance, or non-verbal intelligence (PIQ). In an analysis of subscale scores they found that most verbal subscale mean scores were higher for disomies, whereas the mean Object Assembly subscale score was higher for deletions. Of note is that, in contrast with the usual emphasis on disability, the consensus diagnostic criteria contain a 'supportive' item: unusual skill with jigsaw puzzles. Recently this has been investigated by Dykens (2001). She has suggested that those with PWS due to chromosome 15 deletions exhibit enhanced visuo-spatial skills needed for assembling jigsaw puzzles, and in a test of jigsaw puzzle skill found that, for those with deletions but not for those with disony, these skills exceeded those of non-intellectually impaired children of the same age. As part of our epidemiological study of PWS (Whittington et al. 2001), we invited. all individuals in the region studied who were nominated as possibly or definitely having PWS to participate in a study of the PWS phenotype. The study was based on a population of approximately fure million and gave an estimated birth incidence of $1: 20,000$ to $1: 28,000$, with estimated prevalence and mortality rates of about 1:52,000 and 3\% per year in the population studied. Thirty-one of 123 nominees did not wish to participate in the phenotypic study. We have no reason to suppose that they differed in the variables studied from those who did participate and who comprised our PWS population sample. The findings in this study are based upon 55 participants from the population-based sample, augmented where appropriate with data from 37 people with PWS from outside the region. All were confirmed as having PWS by genetic testing and were older than three years. Various aspects of the phenotypic study have already been reported (Boer of al. 2002; Butler ef al. 2002; Clarke et al. 2002; Whittington et al 2002). All participants in the phenotypic study over the age of three years were administered age-appropriate Wechsler ability scales and (for ages 8 and above) tests of reading, spelling and arithmetic.

In this paper on cognitive function we concentrate on ability distributions, cognitive profiles, the ranges of attainments, and differences between the main genetic subtypes of PWS; a second paper will deal with underachievement and its predictors. This paper also aims to address theoretical issues as well as to report psychometric results. We ask what mechanism(s) might account for the distribum tion of general ability in the PWS population sample, and whether there are systematic cognitive differences between genetic subtypes and, if so, what these differences tell us about genetic influences on cognition, and what implications (if any) this might have for understanding cognitive development in the general population. We have investigated the extent to which our data supports the presence of an exceptional skill (jigsaw puzzles) in the deletion subtype of PWS. Psychometrically, we have looked at general ability, ability profiles and attainment in reading, spelling and arithmetic in PWS, for our population-based sample and 
for the augmented sample, and compared these with published noms and with thos of a learning disabled group of mixed aetiology. We have looked at correlations with age and BMI for any systematic influences on cognition. We have compared ability profiles in the deletion and disomy groups and with the LD group. We hypothesised that there would be a global downward shift in Full Scale IQ of a similar degree in those with PWS due to chromosome 15 disomies and chromosome 15 deletions, but that there would be specific differences in cognitive profiles between the two sub-types that would reflect subtly different genetic infuences on brain development in the two groups.

\section{Method}

Participants. The people identified in the population prevalence study were asked. to participate in a study of the PWS phenotype. Others with PWS from outside the area covered by the population study augmented this cohort. A comparison group (LD) with learning disabilies of mixed aetiologies was recruited from local schools, residential homes and via professional contacts (Whittington et al. 2001). Ethical approval was granted for both studies and consent was obtained from all participants.

Instrmments. Cognitive abilities were assessed by the age-appropriate Wechsler Intelligence Scales [WPPSI-R, 1990, for ages 4-7; WTSC-1ll UK, 1992, for ages 8-16; WAIS-R UK, 1986 for ages 17 and above] and either the Wechsler achievement battery of tests [WORD, 1993; WOND, 1995; WOLD, 1995] (ages 8-16) or the WRAT3 (1993) (ages 1.7 and above). A semi-structured interview schedule, designed for the phenotypic study, elicited information on the presence of clinical diagnostic criteria, a range of behaviours, schooling, medical conditions and medication (Whittington ef al. 2001).

Pracedure. The participants were seen in their place of residence by the same Chartered Psychologist who administered the age-appropriate Wechsler Intelligence Scales and either the Wechsler achievement battery of tests (ages 8-16) or the Wide Range Achevement Tests (ages 17 and above). The main carer was interviewed at the same time, where possible, using the semi-structured interview schedule.

Analyses. Anyone scoring bellow the cut-off level of 45 on both the verbal and performance scales of the Wechsler 19 tests was given a score of 40 . Scores below the respective cut-off points on the Wechsler attainment tests were designated 20 , as were scores below the cut-off of 45 on the WRAT. In some analyses (clearly indicated in text or tables) these low scorers were excluded. In ten cases (3 PWS, 7 LD), people would not fully co-operate or physical disabilities prevented administration of some test items and prorated totals were used to calcullate full-scale $\mathrm{IQ}$, as 
provided in the relevant manuals. To compare relative strengths and weaknesses in cognitive profiles, for each individual we calculated relative subtest score as follows: score on given subtest $\times 100 /$ average score on all subtests. Gender differences in mean FSIQ, VIQ, PIQ and in relative subtest scores were investigated in the augmented PWS group and in the genetic subtypes.

\section{Results}

There were no significant gender differences in mean FSIQ, VIQ, PIQ or in relative subtest scores in the augmented PWS group or in deletion and disomy subtypes ( $t$-tests, $\mathrm{p}=.05)$.

Table 1 shows the means and standard deviations of age, full-scale $I Q$, verbal $1 \mathrm{Q}$, performance IQ, attainment in reading, spelling and arithmetic, and current BMI for the population PWS sample, the full PWS sample, and the comparison LD sample. Table 2 shows the means and standard deviations of the same set of variables for the main genetic subtypes (i.e., deletions and disomies) of PWS. Histograms of frequency distributions for FSIQ, Reading, Spelling and Arithmetic standard scores are shown in Figures 1 to 4 for the population sample, the augmented sample and the comparison LD group. The distribution of IQ is approximately normal for the population PWS sample, but not for the LD sample. The augmented PWS sample has a different IQ distribution which we propose is a consequence of selection bias. The scatterplots (Figure 5) of achievement (reading, spelling and arithmetic) against IQ show that for both children and adults the variations in underachievement are wide. This will be considered further in a later paper. Correlations of age and BMI with FSIQ and the subscales VIQ and PIQ and all subtests are shown in Table 3 for the population PWS and for deletion and disomy population groups; when corrected for the number of tests, none is statistically significant.

Mean standard scores on the subtests of the Wechsler Ability scales for the PWS population sample, the PWS augmented sample, the LD group and the two PWS genetic subtypes are shown in Tables $4-5$. The average general population scores on all subtests is 10 .

Table 6 and Figure 6 compare the cognitive profiles (scores computed as described in Methods) of three groups: the LD group and the two genetic subtypes of PWS. It can be seen that deletion and LD groups are quite similar while the disomy group differs from both. The group with disomies have a cognitive strength, relative to the other two groups, in vocabulary $(p=.001)$, and a weakness in coding (speed) $(\mathrm{p}=01)$.

Table 7 compares the means and standard deviations of the FSIQ, VIQ, PIQ and certain Wechsler Ability subscale scores of five groups: those with deletions 
Table 1. Means, ranges and standard deviations of age, full-scale IO, verball IQ, performance IQ. reading, spelling, arithmetic, and current BMI for the three samples: population PWS, all PWS and comparison Lo group".

\begin{tabular}{|c|c|c|c|c|c|c|c|}
\hline & & \multicolumn{2}{|c|}{ Pop. PWS } & \multicolumn{2}{|l|}{ All PWS } & \multicolumn{2}{|l|}{ LD } \\
\hline & & All IO & $10>40$ & All IO & $10>40$ & Al: IO & $10>40$ \\
\hline \multirow[t]{4}{*}{ Age } & mean & 20.9 & 21.0 & 20.4 & 20.3 & 21.4 & 22.0 \\
\hline & range & $4-46$ & $4-46$ & $4-4.6$ & $4-46$ & $5-53$ & $5 \times 53$ \\
\hline & s.d. & 12.1 & 12.4 & 10.8 & 11.1 & 14.6 & 14.6 \\
\hline & $\mathbb{N}$ & 58 & 52 & 97 & 89 & 55 & 47 \\
\hline \multirow[t]{4}{*}{ FSIO } & mean & 61.4 & 62.6 & 63.6 & 64.4 & 63.5 & 67.0 \\
\hline & range & $40-103$ & $45-103$ & $40-103$ & $45-103$ & $40-107$ & $41-107$ \\
\hline & s.d. & 13.0 & 12.2 & 11.6 & 11.0 & 17.2 & 15.6 \\
\hline & $N$ & 55 & 52 & 92 & 89 & 54 & 47 \\
\hline \multirow[t]{4}{*}{ VIra } & mean & 63.8 & 65.1 & 66.9 & 67.8 & 65.3 & 68.9 \\
\hline & range & $40-101$ & $46-101$ & $40-101$ & $46-101$ & $40-105$ & $46-105$ \\
\hline & s.d. & 13.3 & 12.3 & 12.4 & 11.6 & 17.0 & 15.1 \\
\hline & $\mathbb{N}$ & 55 & 52 & 92 & 89 & 54 & 47 \\
\hline \multirow[t]{4}{*}{ PIO } & mean & 63.1 & 64.4 & 64.1 & 64.9 & 66.1 & 69.8 \\
\hline & range & $40-105$ & $40-105$ & $40-105$ & $40-105$ & $40-109$ & $45-109$ \\
\hline & s.d. & 12.9 & 12.0 & 11.3 & 10.5 & 17.6 & 15.8 \\
\hline & $N$ & 55 & 52 & 92 & 89 & 54 & 47 \\
\hline \multirow[t]{4}{*}{ READING } & mean & 57.2 & 59.1 & 63.0 & 64.3 & 54.6 & 59.7 \\
\hline & range & $20-104$ & $20-104$ & $20 \cdots 106$ & $20-106$ & $20-111$ & $20-111$ \\
\hline & s.d. & 28.0 & 27.7 & 27.0 & 26.5 & 27.8 & 26.1 \\
\hline & $\mathrm{N}$ & 48 & 45 & 81 & 78 & 47 & 41 \\
\hline \multirow[t]{4}{*}{ SPELLING } & mean & 54.3 & 56.6 & 60.3 & 61.9 & 52.0 & 58.0 \\
\hline & range & $20-103$ & $20-103$ & $20-110$ & $20-110$ & $20-114$ & $20-114$ \\
\hline & s.d. & 26.1 & 25.3 & 25.0 & 2.4 .1 & 26.5 & 24.8 \\
\hline & $\mathbb{N}$ & 48 & 45 & 81 & 78 & 47 & 41 \\
\hline \multirow[t]{4}{*}{ ARITH } & mean & 47.5 & 49.4 & 53.3 & 54.6 & 52.0 & 56.7 \\
\hline & range & $20-90$ & $20-90$ & $20-96$ & $20-96$ & $20-117$ & $20-117$ \\
\hline & s.d. & 23.6 & 23.3 & 22.8 & 22.2 & 28.5 & 27.6 \\
\hline & $N$ & 48 & 45 & 81 & 78 & 47 & 41 \\
\hline \multirow[t]{4}{*}{ BMI } & mean & 33.2 & 33.2 & 31.6 & 31.8 & 30.2 & 29.5 \\
\hline & range & $16-76$ & $16-76$ & $16-76$ & $16-76$ & $15-56$ & $16-54$ \\
\hline & s.d. & 12.2 & 12.5 & 11.4 & 11.6 & 10.2 & 9.6 \\
\hline & $N$ & 53 & 49 & 92 & 86 & 52 & 44 \\
\hline
\end{tabular}

* Includes only those with any cognitive data, ie ages greater than 3 
Table 2. Means, ranges and standard deviations of age, full-scale 10 , werbal $1 \mathrm{Q}$, performance 10. attainments in reading, spelling and arithmetic for the genetic subtypes deletions and disomies for the population PWS and all PWS (all IO).

\begin{tabular}{|c|c|c|c|c|c|}
\hline & & \multicolumn{2}{|c|}{ Deletion PWS ([O>40) } & \multicolumn{2}{|c|}{ Disomy PWS (IO $>40)$} \\
\hline & & All & Pop & All. & Pop \\
\hline \multirow[t]{4}{*}{ Age } & mean & 21.7 & 21.2 & 18.4 & 24.6 \\
\hline & range & $5-46$ & $5-46$ & $4-45$ & $6-46$ \\
\hline & s.d. & 11.4 & 13.2 & 12.4 & 14.7 \\
\hline & N & 46 & 28 & 25 & 11 \\
\hline \multirow[t]{4}{*}{ FSIO } & mean & 63.8 & 61.6 & 63.3 & 62.9 \\
\hline & range & $46-91$ & $46-91$ & $46-82$ & $46-82$ \\
\hline & s.d. & 10.2 & 11.2 & 9.7 & 10.4 \\
\hline & $N$ & 46 & 28 & 25 & 11 \\
\hline \multirow[t]{4}{*}{ vio } & mean & 65.8 & 62.4 & 69.7 & 68.8 \\
\hline & range & $46-91$ & $46-91$ & $50-95$ & 50.93 \\
\hline & s.d. & 10.6 & 11.0 & 11.6 & 11.6 \\
\hline & $\mathbb{N}$ & 46 & 28 & 25 & 11 \\
\hline \multirow[t]{4}{*}{ PIO } & mean & 65.9 & 65.6 & 51.05 & 60.3 \\
\hline & range & $47-94$ & $47-94$ & $40-79$ & $40-73$ \\
\hline & s.d. & 9.5 & 10.8 & 8.7 & 9.7 \\
\hline & $\mathbb{N}$ & 46 & 28 & 25 & 11 \\
\hline \multirow[t]{4}{*}{ READING } & mean & 60.2 & 55.6 & 71.3 & 64.7 \\
\hline & range & $20-103$ & $20-103$ & $20-106$ & 20-104 \\
\hline & s.d. & 26.7 & 27.0 & 26.7 & 28.7 \\
\hline & $N$ & 41 & 24 & 21 & 10 \\
\hline \multirow[t]{4}{*}{ SPELLING } & mean & 59.2 & 53.8 & 66.8 & 60.6 \\
\hline & range & $20-96$ & $20-96$ & $20-110$ & 20-103 \\
\hline & s.d. & 24.0 & 24.4 & 24.6 & 25.7 \\
\hline & $\mathbb{N}$ & 41 & 24 & 21 & 10 \\
\hline \multirow[t]{4}{*}{ ARITH } & mean & 54.4 & 51.1 & 53.5 & 45.9 \\
\hline & range & $20-86$ & $20-86$ & $20-90$ & $20-90$ \\
\hline & s.d. & 21.0 & 22.8 & 23.7 & 24.6 \\
\hline & $\mathbb{N}$ & 41 & 24 & 21 & 10 \\
\hline \multirow[t]{4}{*}{ BMI } & mean & 33.3 & 34.6 & 28.5 & 30.8 \\
\hline & range & $16-76$ & $16-76$ & $16-50$ & $21-50$ \\
\hline & s.d. & 13.0 & 14.6 & 8.4 & 8.7 \\
\hline & $N$ & 45 & 27 & 25 & 11 \\
\hline
\end{tabular}

* Includes only those with any cognitive data, ie ages greater than 3 
Table 3. Conclations ( $\mathrm{p}$ values) of $\mathrm{FSIO}_{x} \mathrm{VIO}$, PIO and subtest scores with age and BMI for population PWS, deletion and disomy groups".

\begin{tabular}{|c|c|c|c|c|}
\hline & & Pop PWS & Detetion & Disomy \\
\hline \multirow{2}{*}{ FSIO } & age & $.25(.07)$ & $.41(.03)$ & $.16(.64)$ \\
\hline & $B M I$ & $.16(.28)$ & $.15(.45)$ & $.51(.11)$ \\
\hline \multirow[t]{2}{*}{ VIO } & age & $.16(.26)$ & $.30(.12)$ &. $.03(.94)$ \\
\hline & BMI & $.10(.50)$ & $.07(.74)$ & $.45(.16)$ \\
\hline \multirow[t]{2}{*}{ Plo } & age & $.23(.10)$ & $.40(.04)$ & $.24(.47)$ \\
\hline & BMI & $.11(.45)$ & $.08(.69)$ & $.54(.09)$ \\
\hline \multicolumn{5}{|l|}{ Subtests } \\
\hline \multirow[t]{2}{*}{ Information } & age & $-.23(.10)$ & $-.28(.15)$ & $-.10(.76)$ \\
\hline & BMI & $-.20(.18)$ & $-.34(.08)$ & $.19(.58)$ \\
\hline \multirow[t]{2}{*}{ Similarities } & age & $.12(.40)$ & $.35(.07)$ & $-.37(.27)$ \\
\hline & $\mathrm{BMI}$ & $.04(.77)$ & $.12(.55)$ & $.11(.75)$ \\
\hline \multirow[t]{2}{*}{ Arithmetic } & age & $-.01(.93)$ & $-.07(.73)$ & $-.16(.64)$ \\
\hline & $\mathrm{BMI}$ & $.16(.26)$ &. $.04(.83)$ & $.59(.06)$ \\
\hline \multirow[t]{2}{*}{ Vocabulary } & age & $.18(.20)$ & $.33(.09)$ & $.05(.88)$ \\
\hline & BMI & $.02(.89)$ & $.06(.76)$ & $.14(.68)$ \\
\hline \multirow[t]{2}{*}{ Comprehension } & age & $.21(.13)$ & $.32(.10)$ & $.28(.40)$ \\
\hline & BMI & $.17(.25)$ & $.17(.39)$ & $.51(.11)$ \\
\hline \multirow[t]{2}{*}{ Digit Span } & age & $-.12(.44)$ & $-.10(.157)$ & $-.33(.33)$ \\
\hline & BMI & $-.02(.91)$ & $-.11(.65)$ & $.04(.90)$ \\
\hline \multirow[t]{2}{*}{ Picture Compltion } & age & $-.04(.77)$ & $.00(.98)$ & $-.12(.72)$ \\
\hline & BMI & $.04(.80)$ & $.01(.96)$ & $.28(.41)$ \\
\hline \multirow[t]{2}{*}{ Picture Arrangement } & age & $.24(.09)$ & $.41(.03)$ & $-.04(.90)$ \\
\hline & BMI & $.23(.12)$ & $.27(.17)$ & $.48(.14)$ \\
\hline \multirow[t]{2}{*}{ Block Design } & age & $.114(.31)$ & $.28(.15)$ & $.02(.97)$ \\
\hline & BMI & $.06(.67)$ & $.02(.92)$ & $.36(.28)$ \\
\hline \multirow[t]{2}{*}{ Dbject Assembly } & age & $-.31(.03)$ & $-.25(.19)$ & $-.33(.32)$ \\
\hline & BMI & $-.08(.61)$ & $-.25(.20)$ & $.61(.05)$ \\
\hline \multirow[t]{2}{*}{ Coding } & age & $-.16(.30)$ & $-.09(.66)$ & $-.23(.53)$ \\
\hline & $\mathrm{BMI}$ & $.10(.53)$ & $.03(.86)$ & $.66(.04)$ \\
\hline
\end{tabular}

* age $>3$ years

reported by an informant to have exceptional skils with jigsaw puzzles, those with deletions said not to have exceptional skills with jigsaw puzzles, those with disomies said to have exceptional skills with jigsaw puzzles, those with disomies said not to have exceptional skills with jigsaw puzzles and those with learning disabilities not due to PWS. None of this last group was said by mothers to have exceptional skill with jigsaw puzzles. In all groups, those people in which informants said the person had some skill, but it was not exceptional, were onitted for the purposes of this analysis. Our data suggest that not all those with deletions have similar elevated visuo-spatiall skills, but that visuo-spatial abilities is an area of relative strength in those people with reported jigsaw puzzle skills. However, strikingly, even these people were surpassed on the Object Assembly subtest by the LD group; a surprising result if jigsaw puzzle skills are innate in this PWS subtype. 
Table 4. Mean Subscale scores for PWSpop PWSaug and LD groups

\begin{tabular}{|c|c|c|c|c|c|c|c|}
\hline & & \multicolumn{2}{|c|}{ Pop. PWS } & \multicolumn{2}{|c|}{ All PWS } & \multicolumn{2}{|l|}{10} \\
\hline & & All IO & $I 0>40$ & All IQ & $10=40$ & All Io & $\mathrm{ra}>40$ \\
\hline \multirow[t]{4}{*}{ Info } & mean & 3.3 & 3.4 & 3.7 & 3.8 & 3.9 & 4.2 \\
\hline & range & $1-9$ & $1-9$ & $1-12$ & $1-12$ & $1-14$ & $1-14$ \\
\hline & s.d. & 2.5 & 2.5 & 2.7 & 2.7 & 3.2 & 3.2 \\
\hline & $\mathrm{N}$ & 56 & 53 & 93 & 90 & 51 & 46 \\
\hline \multirow[t]{4}{*}{ Simi } & mean & 5.2 & 3.2 & 5.7 & 5.9 & 5.0 & 5.5 \\
\hline & range & $1-16$ & $1-16$ & $1-16$ & $1-16$ & $1-12$ & $1-12$ \\
\hline & s.d. & 3.3 & 3.2 & 3.0 & 2.9 & 3.2 & 3.0 \\
\hline & $N$ & 55 & 52 & 92 & 89 & 49 & 44 \\
\hline \multirow[t]{4}{*}{ Arith } & mean & 3.3 & 3.5 & 3.7 & 3.7 & 3,4 & 3.7 \\
\hline & range & $1-12$ & $1-12$ & $1-12$ & $1-12$ & $1-13$ & $1-13$ \\
\hline & s.d. & 2.6 & 2.7 & 2.5 & 2.5 & 2.8 & 2.9 \\
\hline & $\mathbf{N}$ & 56 & 53 & 93 & 90 & 51 & 46 \\
\hline \multirow[t]{4}{*}{ Vocab } & mean & 3.6 & 3.7 & 4.2 & 4.3 & 3.9 & 4.3 \\
\hline & range & $1-9$ & $1-9$ & $1-10$ & $1-10$ & $1-10$ & $1-10$ \\
\hline & s.d. & 2.4 & 2.4 & 2.3 & 2.3 & 2.7 & 2.6 \\
\hline & $\mathbb{N}$ & 56 & 53 & 93 & 90 & 50 & 45 \\
\hline \multirow[t]{4}{*}{ Comp } & mean & 4.1 & 4.3 & 4.5 & 4.6 & 4.2 & 4.6 \\
\hline & range & $1-12$ & $1-12$ & $1-12$ & $1-12$ & $1-10$ & $1-10$ \\
\hline & s.d. & 2.7 & 2.7 & 2.6 & 2.5 & 2.6 & 2.5 \\
\hline & $N$ & 56 & 53 & 93 & 90 & 4.8 & 43 \\
\hline \multirow[t]{4}{*}{ DSpan } & mean & 3.0 & 3.1 & 3.4 & 3.5 & 4.0 & 4.3 \\
\hline & range & $1-8$ & $1-8$ & $1-10$ & $1-10$ & $1-14$ & $1-14$ \\
\hline & s.d. & 2.3 & 2.3 & 2.3 & 2.3 & 3.0 & 3.0 \\
\hline & $N$ & 45 & 43 & 81 & 79 & 42 & 39 \\
\hline \multirow[t]{4}{*}{ Picc } & mean & 4.3 & 4.4 & 4.4 & 4.5 & 4.6 & 5.0 \\
\hline & range & $1-12$ & $1-12$ & $1-12$ & $1-12$ & $1-12$ & $1-12$ \\
\hline & s.d. & 2.9 & 2.8 & 2.5 & 2.5 & 3.0 & 2.9 \\
\hline & $N$ & 56 & 53 & 93 & 90 & 50 & 45 \\
\hline \multirow[t]{4}{*}{ Pica } & meian & 3.5 & 3.6 & 4.0 & 4.1 & 4.3 & 4.7 \\
\hline & range & $1 * 11$ & $1 \times 11$ & $1-11$ & $1: 11$ & $1-16$ & $1-16$ \\
\hline & s.d. & 2.4 & 2.4 & 2.3 & 2.3 & 3.9 & 3.9 \\
\hline & $N$ & 56 & 53 & 93 & 90 & 50 & 45 \\
\hline \multirow[t]{4}{*}{ Block } & mean & 4.3 & 4.5 & 4.4 & 4.5 & 4.0 & 4.4 \\
\hline & range & $1-12$ & $1-12$ & $1-12$ & $1-12$ & $1-11$ & $1-11$ \\
\hline & s.d. & 2.9 & 2.8 & 2.7 & 2.6 & 3.1 & 3.0 \\
\hline & $N$ & 56 & 53 & 93 & 90 & 51 & 46 \\
\hline \multirow[t]{4}{*}{ ObjA } & mean & 4.2 & 4.4 & 4.3 & 4.4 & 5.1 & 5.5 \\
\hline & range & $1-12$ & $1-12$ & $1-12$ & $1-12$ & $1-13$ & $1-13$ \\
\hline & s.d. & 2.8 & 2.7 & 2.6 & 2.6 & 3.5 & 3.4 \\
\hline & $\mathbb{N}$ & 56 & 53 & 93 & 90 & 50 & 45 \\
\hline \multirow[t]{4}{*}{ Code } & mean & 3.5 & 3.6 & 3.8 & 3.9 & 3.8 & 4.1 \\
\hline & range & $1-14$ & $1-14$ & $1 \times 14$ & 1.14 & $1-10$ & $1 \times 10$ \\
\hline & s.d. & 3.1 & 3.1 & 2.7 & 2.7 & 2.3 & 2.3 \\
\hline & $N$ & 48 & 45 & 81 & 78 & 45 & 41 \\
\hline
\end{tabular}


Table 5. Mean Subscale scores for PWSpop and PWSaug deletion and disomy groups

\begin{tabular}{|c|c|c|c|c|c|}
\hline & & Deletion (I & & Disomy $(\mathrm{IO}$ & \\
\hline & & Population & All & Population & All \\
\hline Into & mean & 3.0 & 3.4 & 3.2 & 4.0 \\
\hline & range & $1 \times 7$ & $1-11$ & $1-8$ & $1-12$ \\
\hline & s.d. & 2.1 & 2.4 & 2.3 & 3.0 \\
\hline & $\mathbb{N}$ & 28 & 46 & 11 & 25 \\
\hline $\sin 1$ & mean & 4.6 & 5.4 & 6.4 & 5.9 \\
\hline & range & $1-11$ & $1-11$ & $1-10$ & $1-11$ \\
\hline & s.d. & 2.8 & 2.7 & 2.5 & 2.7 \\
\hline & $N$ & 28 & 46 & 11 & 25 \\
\hline Arith & mean & 3.1 & 3.54 & 3.7 & 3.8 \\
\hline & range & $1-12$ & $1-12$ & $1 \cdot 10$ & $1-10$ \\
\hline & s.d. & 2.4 & 2.2 & 2.8 & 2.5 \\
\hline & $N$ & 28 & 46 & 11 & 25 \\
\hline Vocalts & mean & 3.1 & 3.8 & 4.6 & 5.0 \\
\hline & range & $1-9$ & $1-9$ & $1-8$ & $1-10$ \\
\hline & s.d. & 2.1 & 2.1 & 2.3 & 2.2 \\
\hline & $\mathbb{N}$ & 28 & 46 & 11 & 25 \\
\hline Comp & mean & 3.7 & 4.2 & 4.8 & 4.8 \\
\hline & range & $1-8$ & $1-8$ & $1-10$ & $1-11$ \\
\hline & s.d. & 2.3 & 2.2 & 2.6 & 2.7 \\
\hline & $N$ & 28 & 46 & 11 & 25 \\
\hline DSpan & 1 mean & 2.8 & 3.3 & 2.4 & 3.3 \\
\hline & range & $1-8$ & $1-8$ & $1-5$ & $1-10$ \\
\hline & s.d. & 2.2 & 2.1 & 1.0 & 2.4 \\
\hline & $N$ & 21 & 39 & 11 & 24 \\
\hline PicC & mean & 4.6 & 4.6 & 3.6 & 4.0 \\
\hline & range & $1-11$ & $1-11$ & $1-6$ & $1-9$ \\
\hline & 5.d. & 2.5 & 2.1 & 1.4 & 1.9 \\
\hline & N & 28 & 46 & 11 & 25 \\
\hline PicA & mean & 3.3 & 4.0 & 3.6 & 3.8 \\
\hline & range & $1-7$ & $1-7$ & $1-8$ & $1-9$ \\
\hline & s.d. & 2.1 & 2.1 & 2.4 & 2.2 \\
\hline & $N$ & 28 & 46 & 11 & 25 \\
\hline Block & mean & 4.4 & 4.4 & 3.8 & 3.6 \\
\hline & range & $1-9$ & $1-9$ & $1-8$ & $1-8$ \\
\hline & s.d. & 2.6 & 2.3 & 2.4 & 2.3 \\
\hline & $N$ & 28 & 46 & 11 & 25 \\
\hline ObjA & mean & 4.5 & 4.3 & 2.6 & 3.6 \\
\hline & range & $1-9$ & $1-9$ & $1-6$ & $1-8$ \\
\hline & s.d. & 2.4 & 2.3 & 1.9 & 2.5 \\
\hline & $\mathbb{N}$ & 28 & 46 & 11 & 25 \\
\hline Code & mean & 3.8 & 4.1 & 1.9 & 2.7 \\
\hline & range & $1-13$ & 1.13 & $1-4$ & $1-8$ \\
\hline & s.d. & 3.0 & 2.6 & 1.1 & 2.0 \\
\hline & $N$ & 24 & 41 & 10 & 21 \\
\hline
\end{tabular}


Table 6. Cognitive profiles of Deletion, Disomy and $L D$ groups ( $(\mathrm{Q}>40)$ : Mean relative scoves on subtests

\begin{tabular}{|c|c|c|c|c|}
\hline & & Delletion & Disony & Lo \\
\hline \multicolumn{5}{|l|}{ Verbal Scalle Tests } \\
\hline \multirow[t]{3}{*}{ Information } & score & 79.6 & 93.1 & 88.7 \\
\hline & $\mathrm{sd}$ & 43.9 & 46.0 & 33.5 \\
\hline & N & 46 & 25 & 46 \\
\hline \multirow[t]{3}{*}{ Similarities } & score & 131.9 & 152.7 & 123.1 \\
\hline & sid & 55.3 & 62.2 & 50.7 \\
\hline & $N$ & 46 & 25 & 44 \\
\hline \multirow[t]{3}{*}{ Arithmetic } & score & 86.9 & 88.9 & 80.3 \\
\hline & sd & 55.2 & 40.2 & 31.1 \\
\hline & $N$ & 46 & 25 & 46 \\
\hline \multirow[t]{3}{*}{ Vocabulary } & sicore & 88.4 & 126.3 & 94.5 \\
\hline & sd & 31.1 & 40.2 & 33.3 \\
\hline & $\mathbb{N}$ & 46 & 25 & 45 \\
\hline \multirow[t]{3}{*}{ Comprehension } & score & 101.2 & 115.6 & 104.0 \\
\hline & sd & 36.7 & 46.8 & 41.1 \\
\hline & $N$ & 46 & 25 & 43 \\
\hline \multirow[t]{3}{*}{ Digit Span } & score & 78.0 & 82.3 & 83.3 \\
\hline & $s d$ & 48.2 & 41.2 & 27.8 \\
\hline & $\mathbb{N}$ & 39 & 24 & 39 \\
\hline \multicolumn{5}{|l|}{ Non-verbal Tests } \\
\hline \multirow[t]{3}{*}{ Picture Completion } & score & 117.0 & 102.6 & 117.6 \\
\hline & sd & 55,6 & 34.0 & 53.7 \\
\hline & $N$ & 46 & 25 & 45 \\
\hline \multirow{3}{*}{ Picture Arrangement } & score & 94.8 & 93.0 & 102.2 \\
\hline & sd & 42.6 & 37.8 & 51.7 \\
\hline & $N$ & 46 & 25 & 45 \\
\hline \multirow[t]{3}{*}{ Block Diesign } & score & 107.1 & 85.9 & 91.3 \\
\hline & sd & 47.7 & 38.5 & 40.7 \\
\hline & $\mathbb{N}$ & 46 & 25 & 46 \\
\hline \multirow[t]{3}{*}{ Object Assembly } & score & 113.3 & 95.9 & 125.8 \\
\hline & sd & 74.7 & 66.7 & 58.7 \\
\hline & $N$ & 46 & 25 & 45 \\
\hline \multirow[t]{3}{*}{ Coding } & score & 95.5 & 64.3 & 90.2 \\
\hline & $s d$ & 38.2 & 32.4 & 34.7 \\
\hline & N & 41 & 21 & 41 \\
\hline
\end{tabular}


Table 7. Cognitive performances of groups of deletion, disomy and LD subtypes said to have, or lack, respectively, skills with jigsaw puzzles

\begin{tabular}{|c|c|c|c|c|c|}
\hline & $\begin{array}{l}\text { Del-Yes } \\
\qquad N=18\end{array}$ & $\begin{array}{l}\text { Del- Ho } \\
N=18\end{array}$ & $\begin{array}{l}\text { Dis-Yes } \\
N=2\end{array}$ & $\begin{array}{l}\text { Dis-No } \\
\text { Num } 18\end{array}$ & $\begin{array}{l}L D(\mathbb{N o}) \\
\mathbb{N}=39\end{array}$ \\
\hline Age & 25.2 & 20.6 & 16.0 & 15.9 & 23.2 \\
\hline$B M I$ & 35.3 & 34.1 & 44.2 & 26.8 & 29.6 \\
\hline Fsra & 65.8 & 62.3 & 60.5 & 63.7 & 65.8 \\
\hline Via & 65.9 & 66.4 & 62.0 & 71.3 & 67.9 \\
\hline PIQ & 69.9 & 61.9 & 64.0 & 60.8 & 68.6 \\
\hline \multicolumn{6}{|c|}{ Mean scaled and relative subtest scores } \\
\hline \multirow[t]{2}{*}{ Information } & 3.1 & 3.5 & 1.5 & 4.4 & 4.2 \\
\hline & 68.8 & 88.6 & 42.2 & 102.7 & 89.0 \\
\hline \multirow[t]{2}{*}{ similarities } & 5.7 & 5.7 & 6.0 & 6.1 & 5.4 \\
\hline & 136.1 & 141.1 & 174.3 & 156.4 & 123.9 \\
\hline \multirow[t]{2}{*}{ Arithmetic } & 3.2 & 3.4 & 3.0 & 4.1 & 3.6 \\
\hline & 74.5 & 89.0 & 78.9 & 93.0 & 81.0 \\
\hline \multirow[t]{2}{*}{ Vocabulary } & 3.8 & 4.0 & 2.5 & 5.3 & 4.3 \\
\hline & 83.3 & 100.0 & 66.6 & 131.2 & 98.4 \\
\hline \multirow[t]{2}{*}{ Comprehension } & 4.2 & 4.4 & 3.0 & 5.0 & 4.6 \\
\hline & 97.11 & 106.8 & 78.9 & 116.9 & 106.5 \\
\hline \multirow[t]{2}{*}{ Digit Span } & 2.9 & 3.4 & 1.0 & 3.6 & 4.1 \\
\hline & 64.2 & 89.6 & 30.0 & 84.9 & 80.5 \\
\hline \multirow[t]{2}{*}{ Pict. Complet. } & 4.7 & 4.4 & 4.0 & 4.0 & 4.8 \\
\hline & 110.0 & 119.2 & 125.4 & 99.1 & 114.7 \\
\hline \multirow[t]{2}{*}{ Pict. Arrange. } & 3.9 & 4.0 & 5.0 & 3.6 & 4.6 \\
\hline & 89.8 & 100.8 & 149.8 & 81.4 & 104.8 \\
\hline \multirow[t]{2}{*}{ Block Design } & 5.7 & 3.1 & 3.5 & 3.5 & 4.2 \\
\hline & 136.5 & 77.8 & 102.1 & 78.6 & 89.5 \\
\hline \multirow[t]{2}{*}{ Object Assembly } & 5.2 & 3.2 & 6.5 & 3.6 & 5.4 \\
\hline & 136.4 & 84.7 & 197.5 & 89.0 & 123.9 \\
\hline \multirow[t]{2}{*}{ Coding } & 4.3 & 4.0 & 2.0 & 3.1 & 4.0 \\
\hline & 97.4 & 95.1 & 54.4 & 69.1 & 91.8 \\
\hline
\end{tabular}



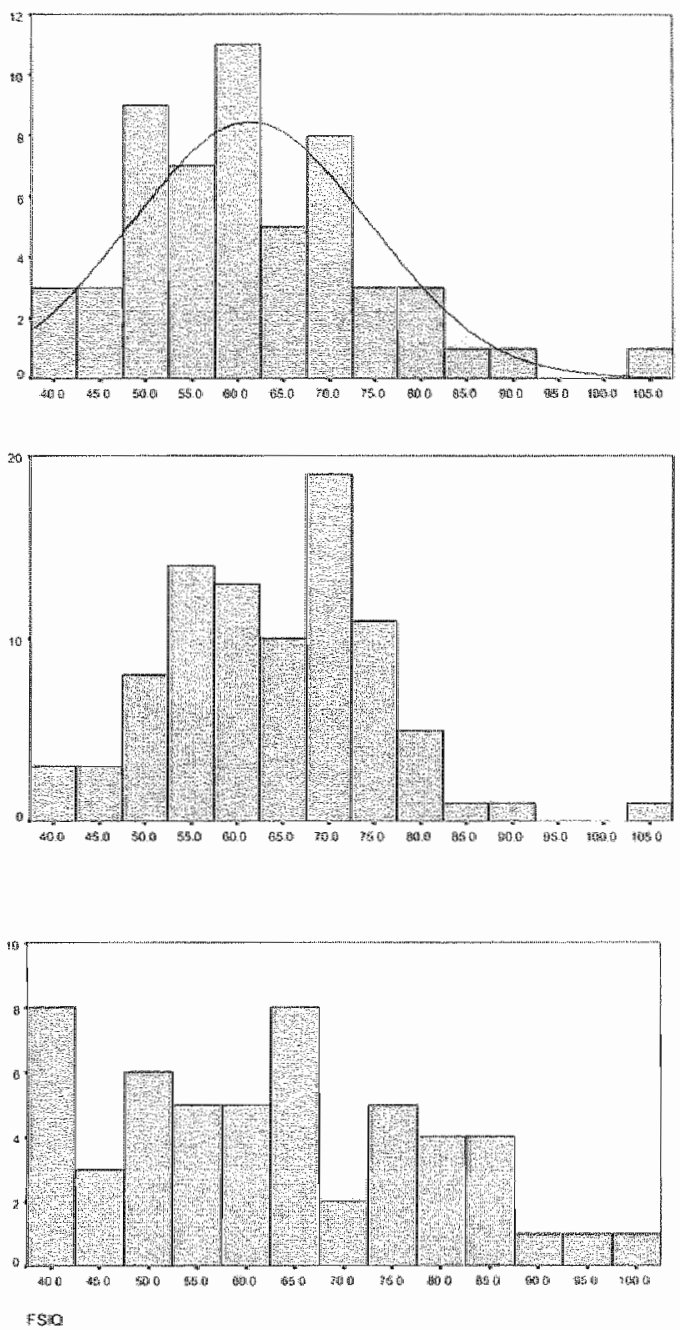

Figure 1. Frequencies of IQ scores for (a) $N=55$ PWS population (b) $N=93$ all PWS (c) $N=5.4$ L.D 

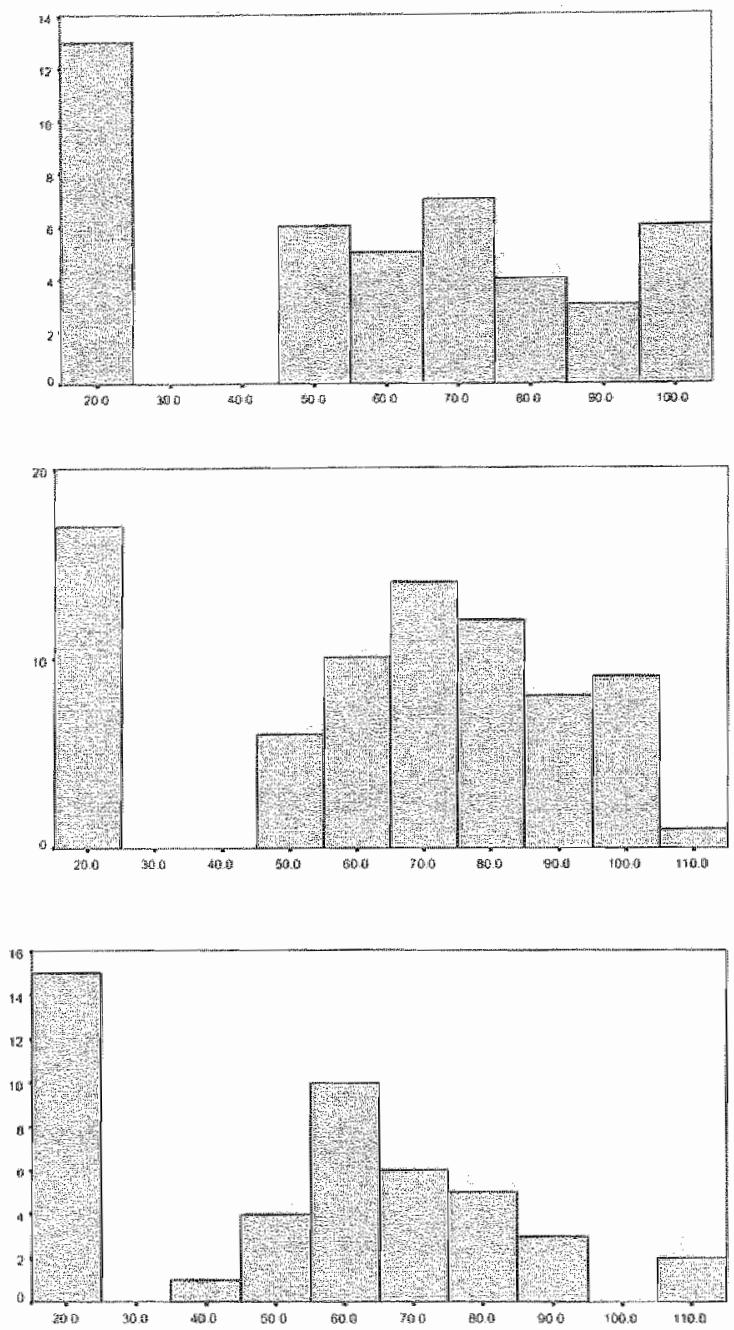

Fisadisng standard seore

Figure 2. Frequencies of Reading attainment standard scores for (a) N=48 PWSpopulation (b) $N=81$ all PWS (c) $N=47 \mathrm{LO}$ 

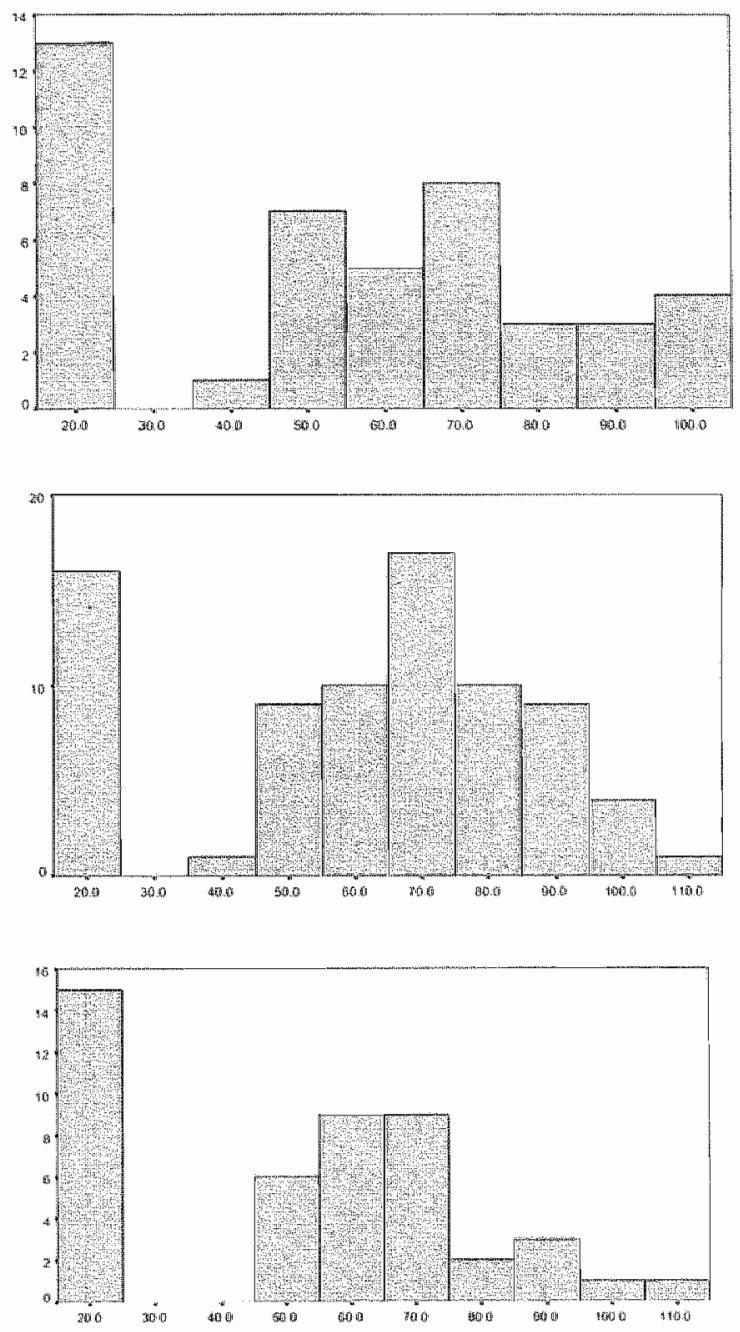

spelling standand score

Figure 3. Frequencies of Spelling attainment standard scores for (a) $N=48$ PWSpopulation (b) $N=81$ all PWS (c) N=47 LD 

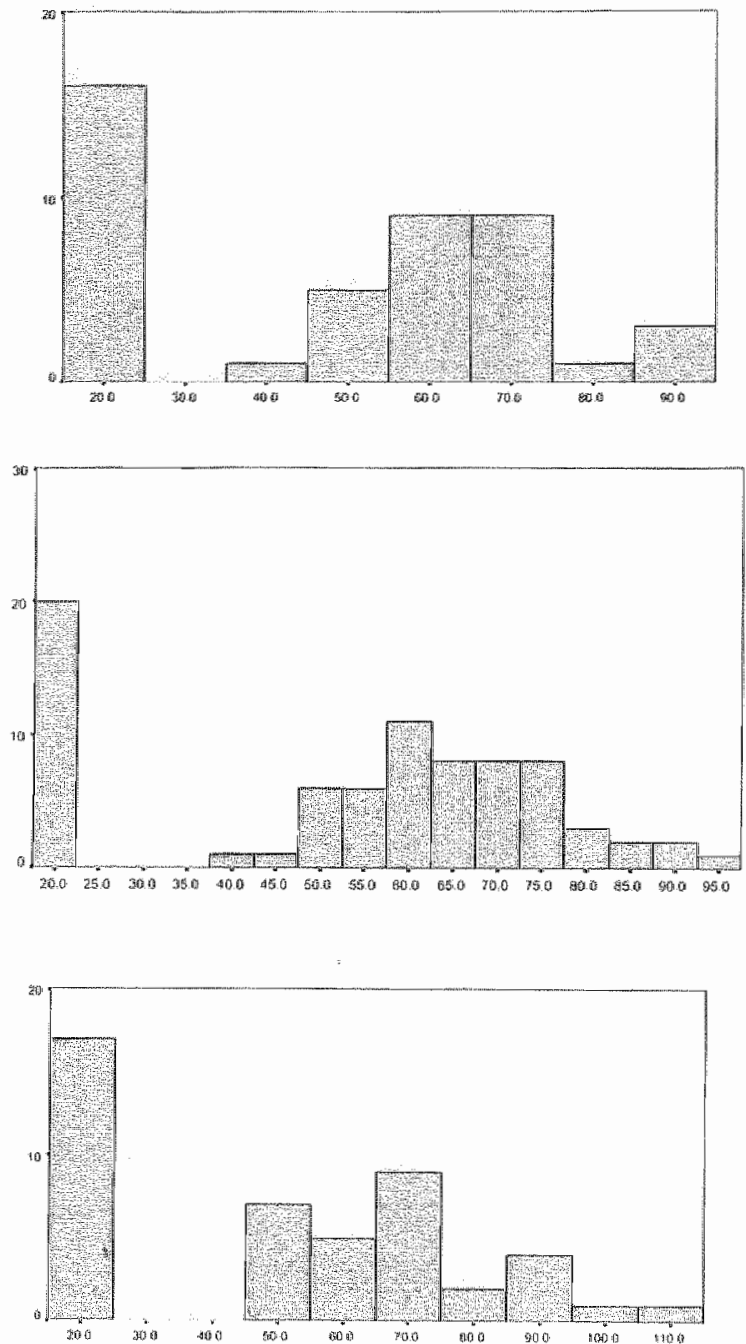

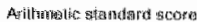

Figure 4. Frequencies of Arithmetic attainment standard scores for (a) $N=48$ PWS population (b) $N=81$ all PWS (c) $N=47 \mathrm{LD}$ 

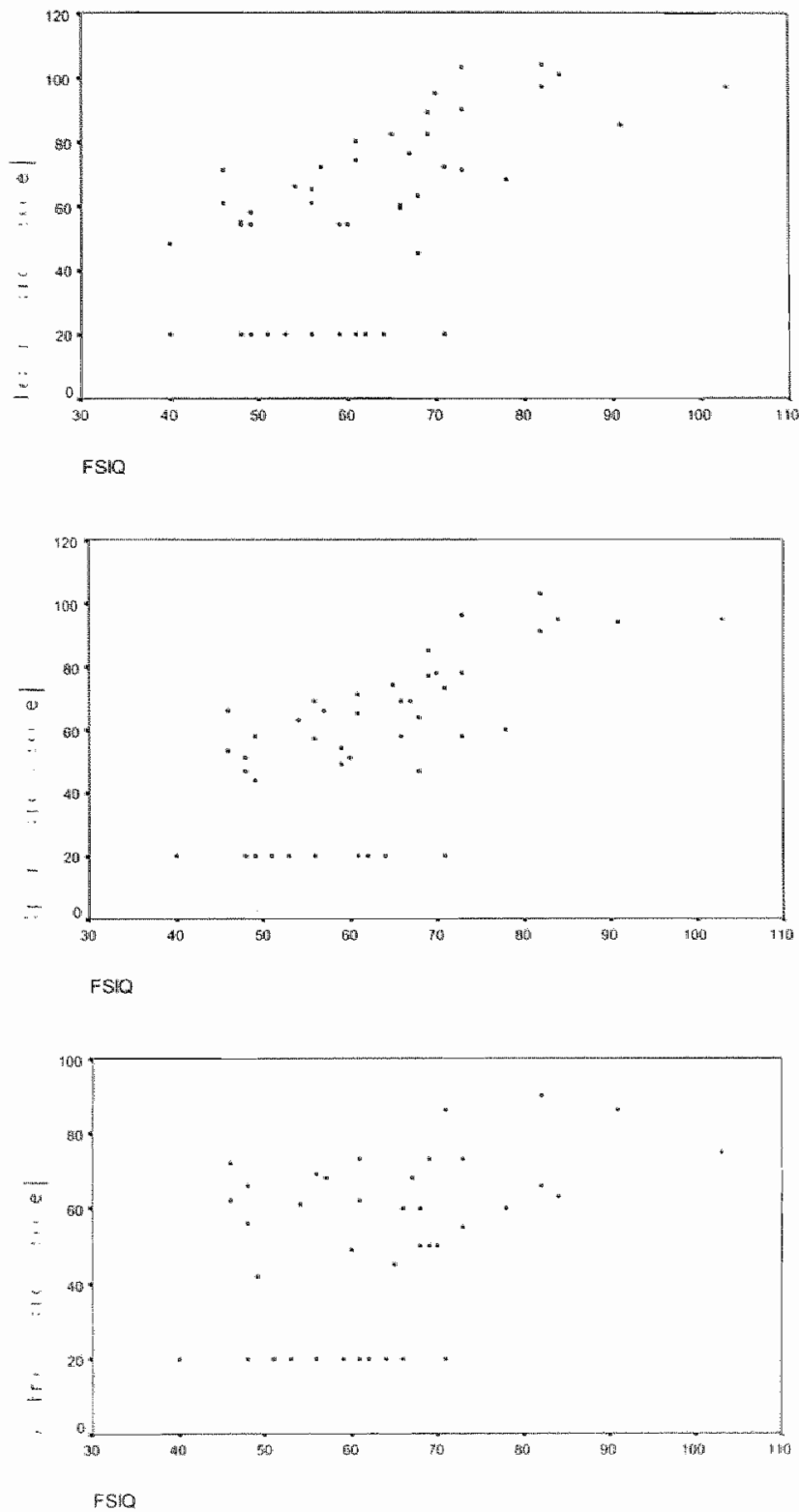

Fïgure 5. Attainments $v$ ability in PWS population sample: (a) Reading (b) Spelling (c) Arithmetic 


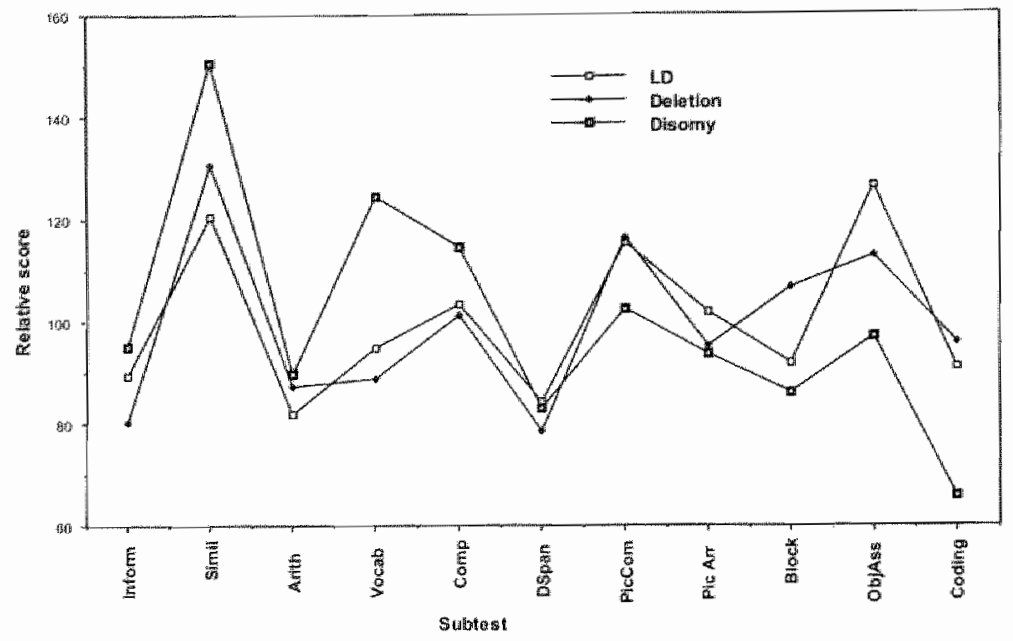

Figure 6. Cognitive Profiles of Deletions, Disomies and the LO group

\section{Discussion}

This is the first population-based study of the cognitive abilities and attainments of children and adults with the genetically determined PWS. As hypothesised, and in keeping with previous reports, we have found a marked downward shift in global ability for both genetic sub-types, together with subtle specific cognitive differences between these two groups. Five people over age three years included in our population study did not wish to participate in the cognitive testing; parental and carer reports would put them in the 50-80 FSIQ range. Three people with PWS and seven in the LD group scored below the Wechsler scale floor on both VIQ and $P I Q$. As the relevant tables show, including these participants by assigning them an $1 Q$ score of 40 or omitting them, makes little difference to the results. Thus, we conclude that those in this sample are representative of the range of abilities and attainments found in people with PWS.

The finding of a near nomal distribution of FSIQ scores for the PWS population suggest that one effect of the PWS genotype is to shift the ability distribution downwards by about $40 \mathrm{IQ}$ points. The fact that all ability subscales are affected, points to a global, rather than a specific effect on cognition. The question of whether this is a systematic (roughly the same for each individual) or a random effect (varying unsystematically between individuals) requires familial studies, and an estimate of heritability of general ability in the PWS population. Some support 
for a systematic effect comes from a recent study of correlations with maternal IQ (Malich et al.2000). This global effect occurs regardless of the specific PWS genotype and therefore we propose that a likely mechanism is that there is a direct and specific effect on brain development due to the absence of expression of an imprinted gene that is part of the PWS genotype. Neither the actual gene that might have this effect nor the underlying pathophysiological consequence is known at present. However, mild cortical atrophy and a small brainstem have been reported and a reduced neuron count in the brains of people with PWS has been hypothesised (Hashimoto et al. 1998).

Our findings support the reports of cognitive differences between deletion and disomy genetic subgroups (Cassidy et al. 1997; Roof ef al. 2000), although there are some differences possibly due to the sampling methods used. We have found higher verbal abilities in those with chromosome 15 disomies (and our results suggest also lower performance abilities), while those with deletions are similar to our general LD group. A new finding is that the disomy group have particular difficulty with coding, and score very poorly on that sub-test.

Reports of people with PWS who have exceptional skill with jigsaw puzzles have appeared in the literature, as anecdotes, with great regularity and 'skill with jigsaw puzzles' has become one of the supportive criteria in the clinical diagnosis of PWS. Several such anecdotes were collected in our population study. Dykens ef al. (2001) conducted an investigation using three groups of children: a group with PWS, an IQ-matched LD group, and an age-matched normal group. Each child was tested on a jigsaw puzzle task (How many pieces could be correctly placed in three minutes), a word-search task and using the block design and object assembly subtests from the WISC. For all tests other than the jigsaw puzzle, the mean scores of the three groups were in the order: normal $>$ PWS $>$ LD; for the jigsaw test, the order was PWS $>$ normal $>$ LD. On dividing the PWS group into those with deletions and those with disomies, the exceptional performance with the jigsaw was found to hold only for the deletion group. Dykens ef al. concluded that deletion PWS is associated with an exceptional skill. However, the effect could be equally well explained on the basis of prior practice. Indeed, given the PWS compulsiveness to repetitive behaviour (especially for word-search tasks and jigsaw puzzles), it seems likely that practice in the PWS group would far exceed that in either of the other groups. Our findings with regard to the two WISC subtests, Block design and Object assembly, show that our PWS group had lower scores than the LD group and far below the average for the general population. However, those with deletions certainly did better than those with disomies (but still no better than the LD group). On dividing the PWS groups according to mother's (or main carer"s) report of exceptional skill with jigsaws, people with deletions said to have such skills were found to perform better on these two WISC subtests. Our interpretation of Dykens's findings is that there may be a practice effect (carers had noticed 
their charges doing jigsaws on many occasions), but that their initial interest in such puzzles nay arise from a natural relative ability in these aspects of visuo-spatial function, that is predominately present in those with deletions.

Overall the IQ profile of those with disomy is different from those with deletion, and from the learning disability control group. Within each genetic group, we expect individual cogmitive profiles to vary, as they do in the general population due to individual genotypic differences, but the consistent difference between genetic subtypes suggests the influence of either over or under expression of a specific maternally or paternally expressed gene on chromosome 15. The two genetic subtypes wil] have a different pattern of expression of maternally imprinted genes located outside the PWS critical chromosomal region, and of paternally expressed genes located either inside or outside this region. We therefore hypothesise not only that failure of expression of a PWS specific gene accounts for the much lower mean full scale IQ score in all people with PWS, but that there are also more subtle effects on brain function and on cognitive performance which is characterised as a better verbal performance of those with disomy, and better visuo-spatial performance for at least some with deletions. In our study there were no significant gender effects, which are known to influence the profile of cognitive abilities, either in the PWS group as a whole or in the genetic subtypes investigated, but we hypothesise that there may be genetic effects on cerebral lateralization that could account for these cognitive differences between genetic sub-types. Further studies should investigate the influence of parental cognitive abilities, sex hormone levels, and other measures of cerebral dominance to try and elucidate further whether such a hypothesis can be sustained or not.

\section{Acknowledgement}

We are grateful to the Wellcome Trust for their support. We would also like to thank the PWS Association for their help contacting families, as well as for further financial support that has enabled this study to continue. Finally, we are particularly grateful to all those people with PWS, their families and other carers for participating in this study.

\section{References}

Boer H., Holland A.J., Whittington., ButlerJ., Webb T., Clarke D. (2002) Psychotic illness in people with Prader-Willi syndrome due to chromosome 15 maternal uniparental disony. The Lance 359, 135-6. 
Buter J.V., Whittington J.E., Holland A.J., Webb T., Butler J.V., Clarke D.J. \& Boer H. (2002) Prevalence of and risk factors for physical ill-hexlth in people with Prader-Willi Syndrome. Developwental Medictie and Child Newrology 44,248-55.

Cassidy S.B., Forsythe M., Heeger S., Nicholls R. D., Schork N., Benn P. \& Schwart2 S. (1997) Comparison of phenotype between patients with Prader-Willi syndrome due to delletion $15 q$ and uniparental disomy 15 . Anserion Joumal of Medical Genefos $68,433-40$.

Cassidy S.B. (1997) Prader-Willi syndrome. Joumal of Medical Gentics 34, 917-23.

Clarke D.J., Boer H., Whittington]., Holland A., Butler ]. \& Webb T. (2002) Prader-Willi syndrome, compulsive and ritualistic behaviours, the first population-based survey. British Jownal of Psychiary 180, 358-62.

Curfs L.M.G. \& Fryns J.-P. (1992) Prader-Willi syndrone; a review with special attention to the cognitive and behavioral profile. Birth Defets: Orgunal Artide Sertes 28, 99-104.

Curfs L.M.G., Wiegers A.M., Sommers J.R.M., Borghgraef M. \& Fryns J.P. (1991) Strengths and weaknesses in the cognitive profile of youngsters with Prader-Willi syndrome. Chincal Generics $40,430-4$.

Dykens E.M., Hodapp R.M., Walsh K. \& Nash L.J. (1992) Protiles, correlates, and trajectories of intelligence in Prader-Willi syndrome. American Academy of Child and Adolestan Pychiary $31,1125-9$

Dykens E.M. (2001) Are jigsaw puzzle skills "spared" in people with Prader-Willi syndrome? Presentation to the 4th Triennial IPWSO Conference, Saint Patil, Minnesota.

Falconer D.S. (1981) Introduction to Quantitatue Getetios. Longnan, New York.

Hashimoto T., Mori K., Yoneda Y., Yanaue T., Miyazaki M., Harada M., Myyoshi H. \& Kuroda $Y$. (1998) Proton magnetic spectroscopy of the brain in patients witlo Prader- Willi syndrome. Pediatric Newrology 18, 30-5.

Holland A.J., Treasure J., Coskeran P., Dallow J., Milton N. \& Hillhouse E. (1993) Measurement of Excessive Appetite and Metabolic Changes in Prader-Willi Syndrome. Internvional Joumal of Obesity 17, 526-32.

Holland A.J., Whitrington J E., Butler J., Webb T., Boer H. \& Clarke D. (2003a) Belhavioural phenotypes associated with specific genetic disonders: evidence from a population based study of people with Prader-Willi syndrome. Psydrological Mediche 33, 141-53.

Holm V.A. (1981) Nedical managenent of Prader-Willi syndrone. In: The Prader-Willi Syndrome.(eds V.A. Holm, S.J. Sulzbacher \& P. L. Pipes), Pp. 221-7. University Patk Press, Baltimore, MD.

Holm V.A., Cassidy S.B., Butler M.G., Hanchet J.M., Greenswag L.K., Whitman B.Y. \& Greenberg F. (1.993) Prader-Willi Syndrome: Consensus Diagnostic Criteria. Pediatric 91 , $398-402$.

Ledbetter D.H., Riccardi V.M., Airhart S.D., Strobel R.J., Keenan B.S. \& Crawford J.D. (1981) Deletion of chromosome 15 as a cause of Prader-Willi syndrome. Netu Eughund Jounal of Mediane 292, 63-6. 
Malich S., Largo R.H., Schinzel A., Molinari L., Eholzer U. (2000) Phenotypic heterogeneity of growth and psychometric intelligence in Prader-Willi syndrome: Variable expression of a contiguous gene syndrome or parent-child resemblance? American Joumal of Medical Cestetics 91, 298-304.

Mitchell J Schinzel A., Langlois S., Gillessen-Kaesbach G., Schuffenhauer S., Michaelis R., Abeliowich D., Lerer 1., Christian S., Guitart M., McFadden D.E. \& Robinson W.P. (1996) Comparison of phenotype in uniparental disomy and deletion Prader-Willi syndrone: sex specific differences. Anevican Journal of Medical Genetis 65, 133-6.

Nicholls R.D. (1993) Genomic imprintangand candidate genes in Prader-Will and Angelman syndromes and mouse models. Chrevt Opimions in Gepetetics and Development 3, 445-56.

Roof E, Stone W., MacLean W., Feurer I.D., Thompson T. \& Butler M.G. (2000) Intellectual characteristics of Prader-Willi syndrome: comparison of genetic subtypes. Joumat of Intellectual Disability Researd 44, 25-30.

Rust I. (1995) Wedrster Objective Language Dimensions (WOLD), Wechster Objective Numerical Distensions (WOND). Psychological Corporation. Harcourt Brace, New York, NY.

Warten H.L. \& Hunt E. (1981) Cognitive processing in children with Prader-Willi syndrome. In The Prader-Will Syndrowe. (eds V.A. Holm, S.). Sulzbacher \& P.L. Pipes), pp. 161-77. Unversity Park Press, Baltimore, MD.

Wechsler D. (1993) The Wedisler Adult Intelligence Sate (WAIS-Revised UK Edition), Wechster Invelligence Sale for Children (WTSC III-UK), Pre-sihool and Primary Sale of Intelligence. (WPPSI-Revised UK), Wedhler Objeative Reading Dimensiows (WORD). Psychological Corporation. Harcourt Brace, New York, NY.

Whittington J.E., Holland A.J., Webb T., ButlerJ.V., Clarke D.J. \& Boer H (2001) Population prevalence and estimated birth incidence and mortality tate for people with Prader-Willi Syndrome in one UK Health Region Jammal of Medical Cenetic 38, 792-8.

Whittington J.E., Holland A.J., Webb T., Butler J.V., Clarke D.J. \& Boer H. (2002) Relationship between clinical and genetic diagnosis of Prader-Willi syndrome. Joumal of Medicat Gerverios $39,926-32$.

Wilkinson G.S. (1993) The Wide Range Adhevement Test (WRAT-3) Psychological Corporam ton. Harcourt Brace \& Co. 
Chapter 3b

\section{Academic underachievement by people with Prader-Willi syndrome}

Published as:

Whittington J., Holland A., Webb T., Butler J., Clarke D. 8. Boer H. (2004) Academic underachievement by people with Prader-Willi syndrome. Joumal of Intellectwal Disability Research $48,188-200$. 


\section{Abstract}

Background. Prader-Willi syndrome (PWS) is a genetically determined neurodevelopmental disorder associated with underwexpression of maternally imprinted genes at the chromosomal locus 15q11-q13. In addition to a characteristic physical and behavioural phenotype, those with the syndrome have impaired social cognition, literal mindedness, and inflexibility. The present authors have investigated the relationship between the PWS cognitive and behavioural phenotype, educational experience, and the levels of attainment in reading, writing, and arithmetic.

Methods. All those from a population-based sample of people with PWS, augmented by those with PWS living in other regions, together with a contrast group of people with learning disabilities of other aetiologies are included in this study. Those children over three years whose families consented or adults who themselves consented were assessed for ability and attainment (over age 7), and information on functional ability was also obtained from an informant. Underachievement was defined as the difference between the score predicted from full-scale $1 Q$ and the actual achievement score.

Findings. Commonly, levels of achevement were lower than would have been predicted on the basis of IQ among those in the group with PWS and LD groups. In the group with PWS under-achievement across academic domains was positively correlated with percentage of time in education in special school, and negatively comelated with Vineland Socialisation domain standard score. There were no across-domain significant correlations in the $L D$ group. When using multiple regression analysis percentage of time in special school was the only predictor of underachievement and only in the group with PWS. However, some PWS children in special schools did acheve as expected in at least one academic domain.

Conchusion. Children with PWS may be placed in special schools largely because of their behavioural problems or physical disabilities or expectations based on their PWS status. Their intellectual abilities may well be masked by their immature social behaviour. The present authors propose that a failure to recognise and address the specific educational needs that follow from this combination of poor socialisation skills and complex maladaptive behaviours, in the context of relativaly mild intellectual impairment, may explain our findings.

\section{Introduction}

It is increasingly recognized that the particular actiology of a leaming disability (LD) may have a specific and characteristic influence on the intellectual, cognitive and emotional development of that child (Dykens et al. 2000). In turn, these 
patterns of cognitive, linguistic and behavioural strengths and weakness maty affect the learning potential, and ultimate attainment of that person. Understanding the individual and his/her unique attributes, together with knowledge of the impact that specific disorder has on his/her development may guide as to the best form of education. As part of a larger study of Prader-Willi Syndrome (PWS) (Whittington et al. 2001), the present authors have explored the level of attainment in a cohort of people with PWS compared to people with learning disabilities due to other aetiologies. The aims of the study reported in this paper were to investigate the extent to which people with PWS reached the attainments predicted by their $I Q$, and to investigate factors that might be associated with any underachievement.

Prader et al. (1956) first described what subsequendy came to be known as PWS, a syndrome of obesity, short stature, crypto-orchidism and mental retardation, following severe hypotonia in the neonatal period. Later, behaviourall characteristics and other clinical features were noted leading to the establishment of the Consensus Diagnostic Criteria (Holm et al. 1993). High rates of both physical and psychiatric morbidity have been observed (Butler ef al. 2002; Boer ef al. 2002), the former being partly a consequence of the severe obesity that can result from not managing the over-eating behaviour. This behaviour starts in early childhood and is one of the main characteristics of the syndrome (Holland ef al. 1993). Most people with PWS have a deletion of part of the long arm of chromosome 15 , with the remainder having other genetic abnormalities (maternal disomy, unbalanced translocations, or defects of the imprinting centre) involving chromosome 15 . The common outcome of all the genetic sub-types is the failure of expression of a number of maternally imprinted genes at 15q11-q13 (Nicholls, 1993).

The literature on cognitive functioning and achievement in PWS is somewhat heterogeneous. People with PWS have been variously described as having "usually mild to moderate mental retardation", "normal intelligence in a significant number of cases", "learning disabilities", "characteristic cognitive weaknesses", "good reading skills", and "weakness in arithmetic skills". Generally, the first two of these descriptions are based on the results of intelligence tests indicating that the IQ is usually in the ranges $50-70$ or $50 \mathrm{~m} 85$ (Curfs et al. 1991; Curfs and Fryns, 1992). As many as one quarter have been found to have an IQ over 70 and one fifth over 85 (depending on the rating system used) (Curfs ot al. 1991; Curfs and Fryns, 1992). The other four descriptions are usually based on discrepancies between IQ subscale scores or on attainment tests (Warren \& Hunt, 1981; Dykens et al. 1992). In the UK, the term "learning disabilities" refers to a global lowering of ability scores, whereas "specific learning disabilities or difficulties" refers to conditions such as dyslexia and dyscalculia in which specific areas of ability are affected, resulting in particular areas of attainment being abnormally low in relation to general IQ. The present authors have proposed that people with PWS have 
both global and specific learning disabilities. The IQ distribution in the PWS population appears to be a normal distribution shified downwards by approximately 40 points below that of the general population (the present authors suspect that this shift is systematic i.e., related to family background IQ) (Whittington et al. 2004), but in addition there are also systematic areas of weakness, such as in arthmetic and social cognition, and also differences in cognitive profile across the two main genetic subutypes ( $R$ oof et al. 2000, Whittington et al. 2004). A recent study by Stauder et al. (2002) supports previous suggestions that in PWS the auditory modality is more affected than the visual and that short-term memory is impaired.

\section{Subjects and methods}

Paricipants. Participants were 55 people aged four years and above with genetically confirmed PWS from our population study (Whittington et al. 2001) and 36 volunteers aged four years and above with genetically confirmed PWS. A comparison wolunteer group of 35 people with learning disabilities (LD) of mixed aetiologies was recruited from local schools, residential homes and via professional. contacts, together with people from the original population study who were found to be genetically and clinically negative for PWS. Mean ages of the PWS and LD groups were 20.4 and 21.2 years (s.d. 10.9 and 1.4 .1 ; ranges $4-46$ and 5-49); mean BMls were 31.5 and 29.1 (s.d. 11.4 and 10.4 ; ranges $16-76$ and 15-56). There were 46 confumed deletions and 26 disomies in the group with PWS who were assessed for this study.

Instruments. Cognitive abilities were assessed by the age-appropriate Wechsler (1993) Intelligence Scales [WPPSI-R, 1990, for ages 4-7; WISC-11L UK, 1992, for anges 8-16; WAIS-R UK, 1986 for ages 17 and abovel, and either the Wechsler achievement battery of tests (WORD-see Wechsler 1993; WOND, WOLD see Rust 1995) (ages 8-16), or the WRAT3 (1993) (ages 17 and above). A semi-structured interview schedule, designed for the main phenotypic study, was used to elicit information on the presence of the clinical diagnostic criteria, a range ofbehaviours, brief screens for Attention Deficit, Hyperactivity and Autistic traits, and developmental, educational, medical and psychiatric histories (Whittingtonet a. 2001).

Predictors. Given some of the specific characteristics of people with PWW the present authors hypothesised that severe behavioural problems, food related behaviours, immature social behaviour, and aberrant behaviours such as temper tantrums and obsessive-compulsive behaviour may all influence level of attainment. Therefore they have included measures of these variables, which had been systematically assessed as part of the larger study. Despite relatively mild intellec- 
tual impaiments the authors noted that many people with PWS had been educated at special schools. The emphasis in these schools is often placed on practical skills of coping with personal needs, domestic tasks and community rules. This approach may not in itself maximise educational skils and therefore we have included percentage of schooling (to date) spent in special needs schools as a vanable. Other variables, which the present authors predicted might affect attainment levels in those with PWS or those in the comparison group, included family size, daytime sleepiness (as measured by scores on the Epworth sleepiness scale Johns, 1991)), attention deficit and hyperactivity, and autism.

Procedure. The participants were seen at their place of residence by the same Chartered Psychologist (JW) who administered the age-appropriate Wechsler Intelligence Scales and either the Wechsler achievement battery of tests (ages $8-16$ years) or the Wide Range Achievement Tests (WRATs; ages 17 and above). Anyone scoring below the cut-offlevel of 45 on the Wechsler $1 Q$ tests was given a score of 40; scores below the respective cut-off points on the Wechsler attainment tests were designated 20 ; and scores below the cut-off of 45 on the WRAT were also designated 20. The informant-based Vineland scales were used to determine functional abilities and socialisation scores (Sparrow et al.. 1984). Aberrant behaviours were measured by the Developmental behaviour Checklist (Einteld \& Tonge 1989) for ages 4-16 years or the Aberrant Behaviour Checklist (Anan et al. 1986) for ages 17 years and above.

A small minority of people (three with PWS and seven with LD) would either not fully co-operate or their physical disabilities prevented administration of some test: items and in this case pro-rated totals were used to calculate full-scale $1 Q$, as recommended in the relevant manuals. The main carers were interviewed by another researcher (J.B.) using a semi-structured interview schedule established for the purpose of the project, standard aberrant behaviour scales, a sleep questionnaire and the Vineland scales.

Underachievement. The manuals for the Wechsler achievement tests each give a able of expected standard scores on the test predicted from the full-scalle IQ score on the WVSC-III. The manual for the WRAT3 gives means and standard deviations for scores of the standardization sample on the WAIS-R, the three attainment subscales and the correlations between them. Separate regression equations of attainment in reading, spelling and arithmetic on $1 Q$ for each of the two age groups were calculated from the above information and used to predict atainment scores from full-scale IQ scores. Actual attainment scores were subtracted from predicted scores to give the degree of underachievement (in standard score units). Underachievement was defined only for 10 scores greater than 40 and for attainment standard scores above 44.

Statistical Analyses. For all the group with PWS and for the group with LD, correlations of underachievement (as defined above) in reading, spelling and arithmetic, 
and the influence of the variables, listed below, were examined. These variables were also entered in multiple regression analyses as explanatory variables with degree of underachievement as the dependent variable. The variables examined were: age, gender, genetic subtype (Gen), number of siblings (Sibs), autistic traits screen score (Aut), attention deficit screen score (AD), hyperactivity screen score $(\mathrm{HA})$, aberant behaviour score (AB), body mass index (BMI), severity of eating abnormality (Eat), daytime sleepiness score (Sleep). Vineland Daily Living Skills domain standard score (VDL), Vineland Socialisation domain standard score (VS), IQ and percentage of schooling spent in special school (SSPC). Finally, the genetic groups, deletion and disomy, were compared on mean underachievement scores.

\section{Results}

Table 1 shows the means and standard deviations of full-scale $I Q$, verbal $1 Q$, performance $1 Q$, attainments in reading, spelling and arithmetic, and underachievement in reading, spelling and arithmetic for the population sample with PWS, the full sample with PWS, and the comparison sample with LD. The population sample is probably more representative of PWS than the usual volunteer and clinic samples. Table 1 shows that volunteers tended to be more able.

Scatterplots of IQ against achievement, for the augmented group with PWS and the group with LD are presented in Figures 1 and 2 , respectively, and show that there were wide variations in (under)achievement for both groups.

Table 2 gives the regression equations for attainment (i.e. reading, spelling, arithmetic) on full-scale IQ score as derived for the respective standardisation samples of the Wechsler and WRAT3 attainment tests. Using these to predict attainment scores from IQ scores, underachievement was calculated as predicted score minus actual score for each of reading, spelling, and arithmetic.

Mean underachievement varies by academic domain and by group. This is shown in Figure 3 where underachievement in reading and, to a lesser extent, spelling is smaller but underachevement in mathematics is similar in the PWS and LD groups.

Table 3 presents correlations of underachevement in reading, spelling and arithmetic with the explanatory variables for the full PWS and the LD cohorts. The correlates of underachievement clearly differ for the groups with PWS and LD. For the group with LJ, no variable affected underachievement across the academic domains but for the group with PWS, both Vineland Socialisation 
Table 1. Means, ranges and standard deviations (SDs) of age, full-5cale $\mathrm{rQ}_{4}$ werbal 10 , performance 10 , current BMI and attainments for the three subject groups: population sample PWS, all PWS and LD*.

\begin{tabular}{|c|c|c|c|c|c|c|c|}
\hline & & \multicolumn{2}{|l|}{ Pop. PWS } & \multicolumn{2}{|l|}{ All PWS } & \multicolumn{2}{|l|}{10} \\
\hline & & All ta & $10>40$ & All I0 & $10>40$ & All I0 & $1 a>40$ \\
\hline \multirow[t]{4}{*}{ FSIO } & mean & 61.4 & 62.6 & 63.5 & 64.3 & 63.4 & 66.4 \\
\hline & range & $40-103$ & $45-103$ & $40-103$ & $45-103$ & $40-99$ & $41-99$ \\
\hline & s.d. & 13.0 & 12.2 & 11.7 & 11.0 & 16.6 & 15.2 \\
\hline & $\mathbb{N}$ & 55 & 52 & 91 & 88 & 35 & 31 \\
\hline \multirow[t]{4}{*}{ vio } & mean & 63.8 & 65.1 & 66.8 & 67.7 & 64.5 & 67.7 \\
\hline & range & $40-101$ & $46-101$ & $40-101$ & $46-101$ & $40-101$ & $46-101$ \\
\hline & s.d. & 13.3 & 12.3 & 12.4 & 11.6 & 16.5 & 14.8 \\
\hline & $\mathbb{N}$ & 55 & 52 & 91 & 88 & 35 & 31 \\
\hline \multirow[t]{4}{*}{ PIO } & mean & 63.1 & 64.4 & 64.0 & 64.8 & 66.5 & 69.9 \\
\hline & range & $40-105$ & $40-105$ & $40-105$ & $40-105$ & $40-105$ & $45-105$ \\
\hline & s.d. & 12,9 & 12.0 & 11.3 & 10.6 & 17.5 & 15.6 \\
\hline & $N$ & 55 & 52 & 91 & 88 & 35 & 31 \\
\hline \multirow[t]{4}{*}{ READING ${ }^{\wedge}$} & mean & 57.2 & 59.1 & 62.9 & 64.2 & 54.8 & 58.8 \\
\hline & range & $20-104$ & $20-104$ & $20-106$ & $20-106$ & $20-91$ & $20-91$ \\
\hline & $5 . d$ & 28.0 & 27.7 & 27.1 & 26.7 & 22.3 & 19.9 \\
\hline & $\mathbb{N}$ & 48 & 45 & 80 & 77 & 29 & 26 \\
\hline \multirow[t]{4}{*}{ SPELLING^ } & mean & 54.3 & 56.6 & 60.1 & 61.7 & 53.0 & 56.8 \\
\hline & range & $20-103$ & $20-103$ & $20-110$ & $20-110$ & $20-86$ & $20-86$ \\
\hline & s.d. & 26.1 & 25.3 & 25.1 & 24.3 & 20.8 & 18.3 \\
\hline & $\mathbb{N}$ & 48 & 45 & 80 & 77 & 29 & 26 \\
\hline \multirow[t]{4}{*}{ ARITHMETIC ${ }^{\wedge}$} & mean & 47.5 & 49.4 & 53.1 & 54.4 & 52.6 & 56.4 \\
\hline & range & $20-90$ & $20-90$ & $20-96$ & $20-96$ & $20-94$ & $20-94$ \\
\hline & s.d. & 23.6 & 23.3 & 22.8 & 22.3 & 25.7 & 24.4 \\
\hline & $N$ & 48 & 45 & 80 & 77 & 29 & 26 \\
\hline \multicolumn{2}{|c|}{ \$READING ${ }^{\wedge}$-undmean } & & 4.9 & & 3.7 & & 12.7 \\
\hline \multirow{2}{*}{\multicolumn{2}{|c|}{$\begin{array}{l}\text { \$SPELLING }{ }^{\wedge} \text {-undmean } \\
\text { \$ARITH^ - undmean }\end{array}$}} & & 11.8 & & 10.6 & & 16.3 \\
\hline & & & 13.8 & & 12.7 & & 12.9 \\
\hline
\end{tabular}

* Includes only those with any cognitive data, i.e., ages greater than 3

"Ages eight and above

\$ Standard Scores above 44 only 


\section{CHAPTER 38}

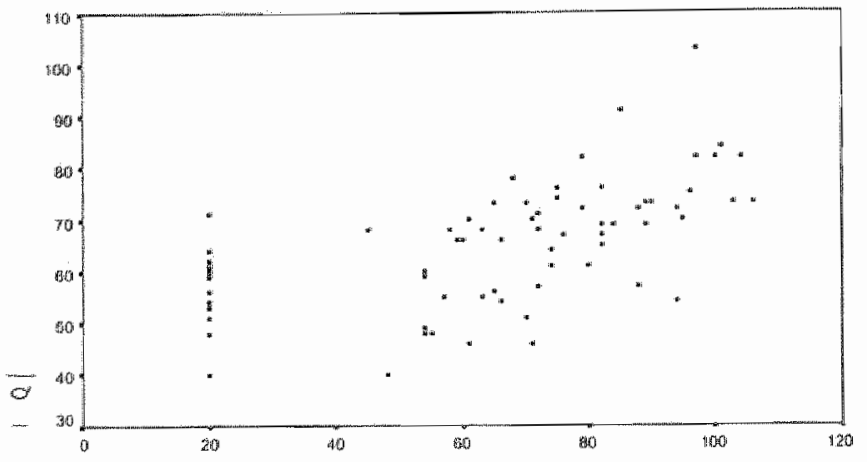

Reading slandard scorom PABS

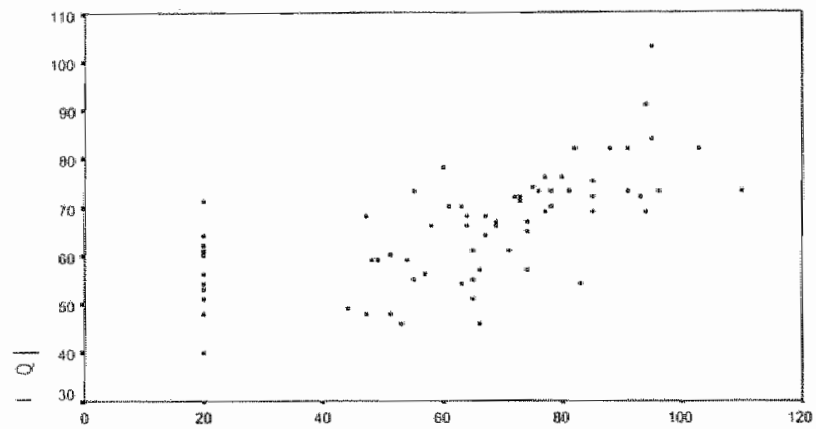

Spoling standard scoiremal PWS

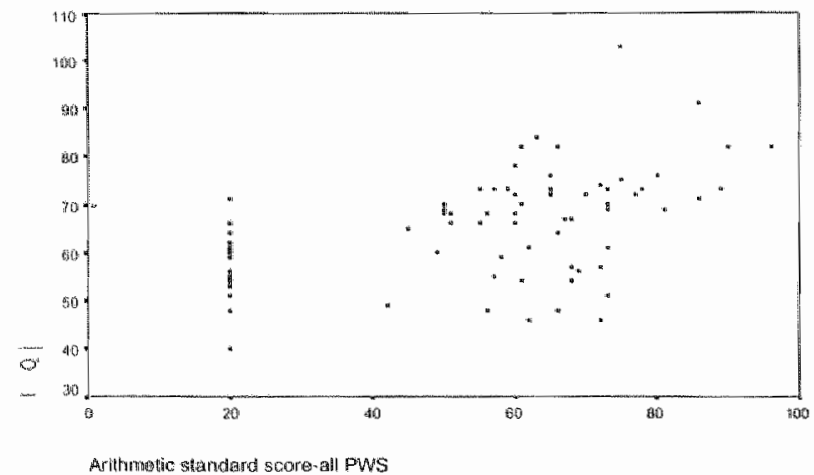

Figure 1. Full-scale Ia scores according to (a) reading (b) spelling (c) arithmetic standard scores for all groups with Prader-Willi syndrome. 


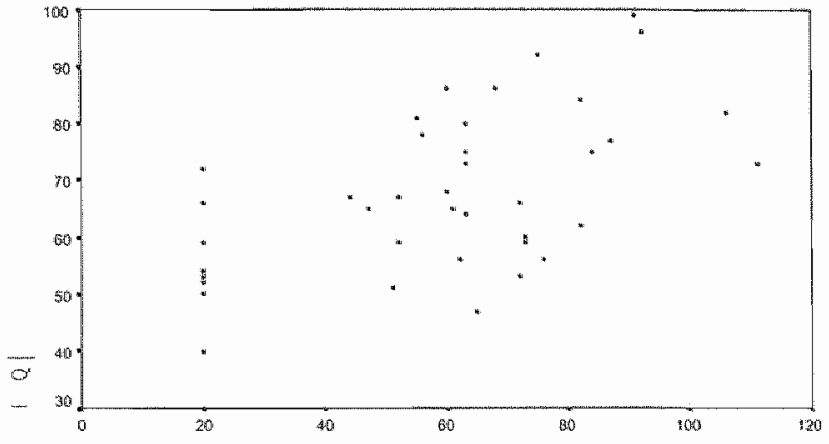

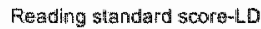
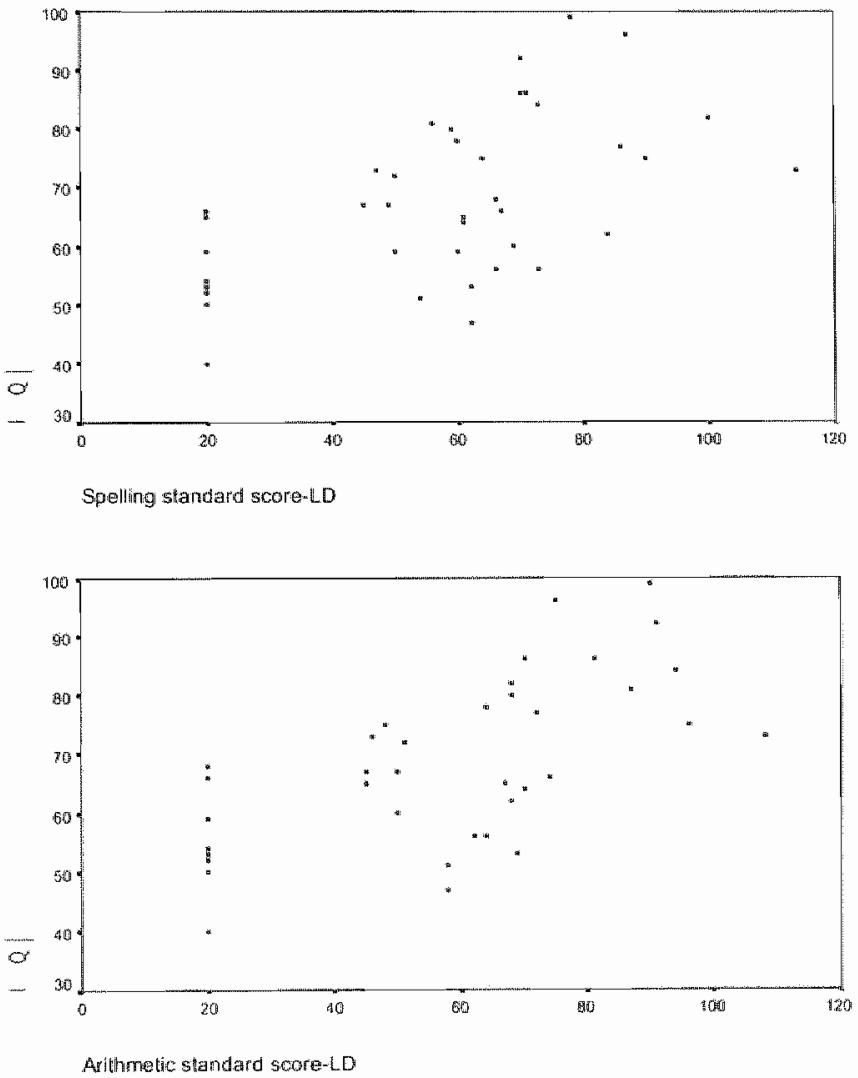

Figure 2. Full-scale IQ scores according to (a) reading (b) spelling and (c) arithmetic standard scores for the group with learning disability. 
Table 2. Regression equations of attainment measures on full-scale IQ derived from relevant Wechsler and WRAT manuals.

\begin{tabular}{ll}
\hline Attainment measure & Regression equation \\
WORD reading & $P($ reading $)=40+0.6 *$ FSIO \\
WORD spelling & $P($ spelling $)=50+0.5 *$ FSIO \\
WOND arithmetic & $P($ arithmetic $)=42.5+0.575 * \mathrm{FSIO}$ \\
WRAT3 reading & $P($ reading $)=51.8+0.424 *$ FSIO \\
WRAT3 spelling & $P($ spelling $)=50+0.446 *$ FSIO \\
WRAT3 arithmetic & $P($ arithmetic $)=37.5+0.559 *$ FSIO \\
\hline
\end{tabular}

Table 3. Correlations of underachievement (predicted standard score minus actual standard score) with explanatory variables for PWS and LD groups

\begin{tabular}{lcccccc}
\hline & \multicolumn{5}{l}{ PWS Group } & \multicolumn{2}{l}{ LD Group } \\
& Read & Spell & Arith & Read & Spell & Arith \\
\hline A.ge & .18 & .10 & $.36^{* *}$ & .35 & .22 & $.55^{* *}$ \\
Sex & -.08 & -.08 & .09 & .12 & .29 & .20 \\
Sibs & -.06 & -.01 & -.03 & .07 & .23 & .10 \\
Gen & -.12 & -.08 & -.03 & .21 & .30 & .09 \\
A.ut & .10 & .23 &.- .09 & -.26 & -.18 & -.23 \\
A.D & -.20 & -.16 & -.05 & -.12 & -.02 & -.02 \\
HA & .06 & .10 & .03 & -.21 & -.11 & -.10 \\
AB child & -.22 & -.32 & -.01 & -.22 & -.20 & .42 \\
& .21 & .35 & .14 & .03 & .12 & -.34 \\
BMI adult & $.31^{*}$ & .20 & .03 & $.38^{*}$ & .31 & .37 \\
Eat & $.27^{*}$ & .23 & .23 & -.05 & -.06 & .02 \\
Sleep & .21 & .11 & .08 & .31 & .22 & .30 \\
VDL & -.11 & -.27 & -.04 & .20 & .04 & -.21 \\
VS & $-.34^{*}$ & $-.39^{* *}$ & $-.39^{* *}$ & .19 & .14 & -.26 \\
I0 & -.24 & $-.49^{* * *}$ & .10 & .09 & .07 &.- .08 \\
SSPC & $.51^{* * *}$ & $.51^{* * *}$ & $.37^{* *}$ & .22 & .33 & .33 \\
\hline
\end{tabular}

* $=$ significant $p=05 ; *$ * significant $p=01_{*}^{*} *$ * $=$ significant $p=001$

SIBS - Number of siblings; GEN $=$ Genetic subtype; $A U T=$ Autistic trailts screen score $\mathrm{AD}=$ Attention deficit screen score; $H A=$ Hyperactivity screen score; $\mathrm{AB}$ = Autistic traits screen score; EAT = Sewerity of eating abnormality; VLDS = Vimeland daily living skills domain standard score; WS = Vineland socialization domain standard Score; SSPC = Schooling spent in special school

domain standard score and percentage of schooling in special school correlated with underachievement across all three academic domains. For both groups, underachievement in arithmetic correlated with age and underachievement in reading correlated with BMI. Using multiple regression analysis for the group with LD there was no significant predictor of underachievement, but for the group with PWS percentage of schoolingspent in special school remained a significant predictor for all three academic domains. 


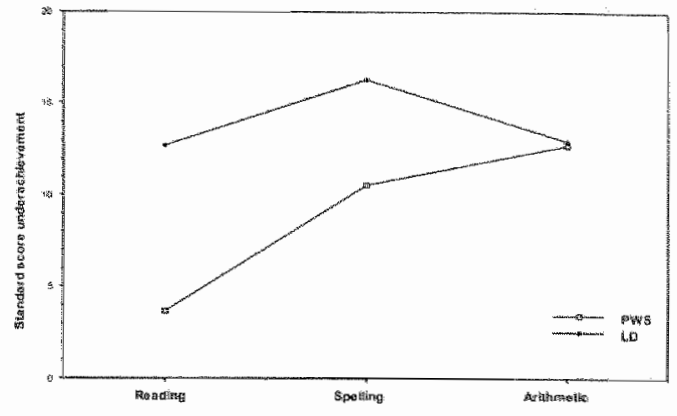

Figure 3. Mean underachievement of PWS and LD groups across academic domains

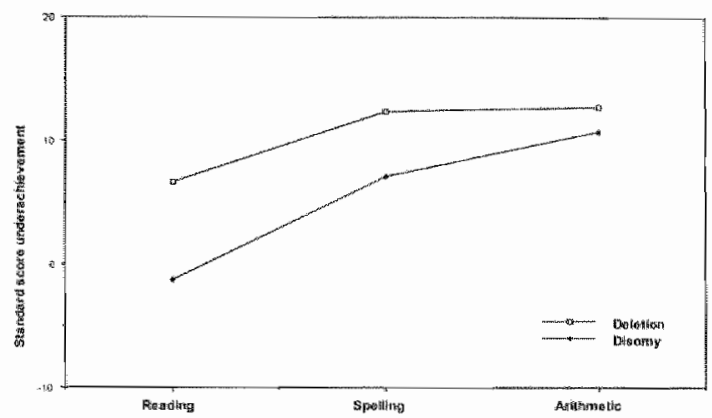

Figure 4. Mean underachievement of PWS genetic subgroups across academic domains

Strikingly, among people with PWS, mean underachievement varied by academic domain and by genetic subgroup (see Figure 4). Differences in mean underachievement between those with deletion and those with disomy are greatest in reading, slightly smaller in spelling, and very small in arithmetic. The disomy subgroup actually reached the mean expected attainment level in reading. The differences in degree of underachievement between the subgroups in reading and spelling were approximately half of the standard deviations within subgroups. 
Table 4. Means, ranges and standard deviations (SDS) of explanatory variables and attainments in reading, spelling and arithmetic for the genetic subtypes deletions and disomies for all PWS

\begin{tabular}{|c|c|c|c|c|c|c|c|c|}
\hline & \multicolumn{4}{|c|}{ Deterion PWS } & \multicolumn{4}{|c|}{ Disomy PWS } \\
\hline & Mean & s.d. & $N$ & Range & Mean & s.d. & $\mathbb{N}$ & Range \\
\hline \multicolumn{9}{|l|}{ Abulities } \\
\hline FSIO & 63.8 & 10.2 & 46 & $46-91$ & 63.4 & 9.6 & 26 & $46-82$ \\
\hline via & 65.8 & 10.6 & 46 & $46-91$ & 69.9 & 11.4 & 26 & $50-95$ \\
\hline PIO & 65.9 & 9.5 & 46 & $47-94$ & 61.2 & 8.6 & 26 & $40-79$ \\
\hline \multicolumn{9}{|l|}{ Attainments } \\
\hline READ & 73.2 & 15.5 & 31 & $45-103$ & 78.2 & 18.3 & 19 & $48-106$ \\
\hline SPELL & 68.7 & 15.6 & 33 & $44-96$ & 74.6 & 16.2 & 18 & $49-110$ \\
\hline ARITH & 64.1 & 11.3 & 32 & $42-86$ & 66.9 & 11.4 & 15 & $50-90$ \\
\hline Und READ & 6.6 & 13.8 & 30 & $-22-36$ & -1.3 & 14.6 & 15 & $-22-23$ \\
\hline UndSPELL & 12.3 & 13.0 & 32 & $-13-33$ & 7.1 & 13.5 & 15 & $-23-27$ \\
\hline UndARITH & 12.7 & 9.8 & 31 & $-3-29$ & 10.8 & 10.8 & 13 & $-7-27$ \\
\hline \multicolumn{9}{|c|}{ Explanatory variables } \\
\hline BMI & 31.8 & 13.1 & 50 & $15-76$ & 27.5 & 8.4 & 29 & $16-50$ \\
\hline EAT & 2.4 & .86 & 49 & $a-3$ & 1.9 & .83 & 29 & $1-3$ \\
\hline $\mathrm{ADD}$ & 3.3 & 2.2 & 48 & $0-8$ & 4.2 & 2.0 & 26 & $0-7$ \\
\hline HO & 2.9 & 1.7 & 48 & $0-6$ & 3.1 & 1.9 & 26 & $0-8$ \\
\hline AUT & 2.8 & 1.8 & 48 & $0-7$ & 3.4 & 2.5 & 26. & 0.8 \\
\hline child & 48.5 & 20.4 & 20 & $17-93$ & 41.8 & 21.6 & 14 & $15-89$ \\
\hline aidult.t. & 25.8 & 16.6 & 30 & $3-59$ & 31.4 & 23.5 & 13 & 6.82 \\
\hline SIBS & 1.56 & .94 & 55 & $0-4$ & 1.60 & 1.19 & 30 & 0.5 \\
\hline SLEEP & 8.6 & 4.7 & 49 & $0-18$ & 8.5 & 5.0 & 28 & $1-18$ \\
\hline SSPC & 55.1 & 41.9 & 32 & $0-100$ & 41,8 & 46.2 & 18 & 0.100 \\
\hline VOLS & 46.8 & 19.6 & 50 & 20.95 & 44.8 & 19.2 & 29 & $20-89$ \\
\hline VS & 48.8 & 16.0 & 51 & $20-89$ & 50.6 & 18.5 & 30 & $20-80$ \\
\hline
\end{tabular}

SLEEP $=$ Daytime sleepress score; 5 SPC $=$ Schooling spent in special school $(\%)$; VDLS $=$ Vineland daily living skills domain standard score: $V S=$ Vineland socialisation standard score.

\section{Discussion}

In this study the present authors have investigated factors that influence educational attainment levels in those with a complex neurodevelopmental disorder. The genotype of PWS clearly has a major effect on the cognicive phenotype and resultant attainments, but there are interesting differences between genetic subtypes and, most importantly, evidence that the nature of the education received affects outcome for people with this syndrome.

There has been much debate in the behavioural phenotype literature as to the best comparison group (e.g., Dykens et al. 1997; Hodapp and Dykens, 2001). The present authors have amalgamated two groups: (1) those who volunteered and were thought to have PWS, but were found not to have the full clinical features or the PWS genotype and, (2) children and adults identified through a local special school and social services provision, respectively. The advantage of such a group is 
Table 5. Percentage of schooling in special needs schools and 10 and numbers at various levels of achievement

Percentage of schooling in special needs schools

$0 \quad 1-50 \quad 51-100$

\begin{tabular}{|c|c|c|c|}
\hline \multicolumn{4}{|c|}{ PWS group $(n=71)$} \\
\hline Number & $21(30 \%)$ & $8(11 \%)$ & $42(59 \%)$ \\
\hline Mean IQ $(\geq 40)$ & 73 & 66.5 & 62 \\
\hline 10 range & $51-103$ & $49-84$ & $46-82(3 \leq 40)$ \\
\hline Read $\geq$ expect & 13 & 3 & 8 \\
\hline Spell 2 expect & 10 & 2 & 2 \\
\hline Arith $\geq$ expect & 9 & 0 & 1 \\
\hline All > expect & 5 & 0 & 0 \\
\hline Read < cut-off & 0 & 0 & 13 \\
\hline Spell < cut-off & 0 & 0 & 12 \\
\hline Arith < culit-off & 0 & 0 & 15 \\
\hline All < cut-off & 0 & 0 & 11 \\
\hline \multicolumn{4}{|c|}{ LD group $(n=40)$} \\
\hline Number & $9(22.5 \%)$ & $6(15 \%)$ & $25(62.5 \%)$ \\
\hline Mean IO $(\geq 40)$ & 82 & $75^{\circ}$ & 60.5 \\
\hline IQ range & $65-99$ & $53-86$ & $41-86(4 \leq 40)^{*}$ \\
\hline Read $\geq$ expect & 4 & 1 & 2 \\
\hline Spell $\geq$ expect & 3 & 0 & 1 \\
\hline Arith 2 expect & 2 & 1 & 0 \\
\hline All $\geq$ expect & 2 & 0 & 0 \\
\hline Read < cut-off & 0 & 0 & 9 \\
\hline Spell < cut-off & 1 & 0 & 8 \\
\hline Arith < cut-off & 0 & 0 & 11 \\
\hline All $<$ cut-off & 0 & 0 & 8 \\
\hline
\end{tabular}

* omitted from IO means and ranges and achievement numbers

that it is not made up of people with one single syndrome, who may have distorted any comparison because of the characteristics of that syndrome. Secondly, a proportion were also obese, and there was a sufficient range of BMI in the comparison group to enable us to examine a relationship between behaviour and $\mathrm{BMI}$ in that group, as well as in those with PWS.

The finding that the percentage of time in schooling spent in special needs schools (time-sns) is a significant predictor of underachievennent in the group with PWS, but not in those with LD, is not easily explained. Anecdotally, it is known that some children with PWS are placed in special needs schools, not because of low $\mathrm{rQ}$, but because of behavioural problems (eg temper tantrums and stealing food or money to buy food) which are not routinely dealt with in mannstream schooling. Correlations between aberrant behaviour and time-sns were 21 (children) and 
.25 (adults) in the group with PWS and .01 and -.08 in the Group with LD, which gives some support to the anecdotal evidence. However, this cannot be the whole explanation because correlations between underachievement and behavioural measures were not significant (Table 3). Further investigation also revealed that IQ differences did not account for time-sns in either group (see Table 5). Significantly, all seven people (five with PWS and two with LD) who were achieving at expected levels in all three academic domains had never, or not yet, been to a special needs school, while out of 19 (11 with PWS and eight with LD) with an IQ $>40$ who were below the cut-off points on all three attainment tests only three subjects had time-sns $<100 \%$ (one with $\mathrm{LD}, 55 \%$; one with PWS transferred at the age of five years; and one with PWS with psychiatric illness whose attainment levels had clearly been higher in the past). On the other hand, as shown in Table 5 , some people who had spent more than half their schooling in special needs schools were achieving at expected levels in at least one academic domain. This seems to indicate that it is not the type of school per se that fosters underachievement, but that some special needs schools are less likely than mainstream schools to teach basic reading, spelling and arithmetic to children who are difficult to accommodate. They may be difficult to accommodate within a particular school because their general ability levels are higher than those of their classmates or appear lower than they really are because of immature behaviour, or because of expectations based on their school placement. Out of the basic skills, reading is the most likely to be tackled in a special needs school and is "a strength of people with PWS", which might explain the number of those in the group with PWS in the time-sns = $51-100 \%$ category who do achieve at expected levels in reading.

Underachievement patterns were different for the groups with PWS and LD. The group with PWS was better at reading than arithmetic, with spelling intermediate, whereas the LD group were equally good at reading and arithmetic and worse at spelling. The group with PWS had lower levels of underachievement in reading and spelling than the LD group, but performance in arithmetic was similar. It seems that, relative to other people with learning disabilities, people with PWS have relative strengths in reading and spelling, but not in arithmetic.

In both the group with PWS and those with LD, underachievement in arithmetic was significantly correlated with $\mathrm{IQ}$. This may indicate that the regression equations derived from the normalisation samples for the Wechsler and WRAT attainment tests break down for the arithmetic test at lower levels of IQ. However, the lack of significant correlations with IQ for underachievement in reading and spelling suggest that the regression equations for these do hold at lower IQ levels. The present authors are unable to offer any explanation of the correlation between underachievement in reading and BMI in both the group with PWS and those 
with LD. The level of significance is not large, so this may just be a consequence of the number of significance tests conducted.

As the scatter plots show, underachievement is not universal in either in the group with PWS or those with LD, but the average attainments are below the predicted levels. Through correlation analysis two systematic influences on underachievement have been identified for the group with PWS: school type, as discussed above, and Vineland socialisation domain standard score. The latter may be interpreted as a proxy for some of the factors hypothesised to be instrumental in achievement in normal children via their influence on the ability to interact with others; for example, motivation, response to encouragement, pleasing others, and competing. The present authors also formed the view that certain other factors may have affected the attainment levels of particular individuals but without any systematic effect in either the group with PWS or those with LD. These factors included gender, number of siblings (e.g. divided attention from their parents or competition), BMI and severity of eating disorder (e.g. intrusive thoughts of food or body image), daytime sleepiness, aberrant behaviour, and attention deficit, hyperactivity and autistic traits. The authors were not able to include some possibly relevant variables such as teaching methods. They suspect that teaching based on concrete materials, rule-based learning and visual methods would be better for people with PWS, who have poor auditory short-term memory, difficulty in generalising, and do not understand abstract concepts.

Finally the present authors have found that the patterns of underachievement across academic domains differ for deletion and disomy genetic subgroups of people with PWS. On average the subgroup with disomy showed no underachievement in reading. The poorer underachievement in the deletion group may be explained by the greater average percentage of schooling spent in special schools or by the higher verbal IQ compared with performance IQ means of the group with deletion relative to those with disomy (see Table 4).

In the UK it is the process of 'statementing' that is the gateway to receiving special educational help. This process may need to consider the cause of a person's disability and its impact on cognitive, social and emotional development if it is to ensure that all people with learning disabilities receive the form of education that results in the best possible outcomes. Whilst the cognitive development of people with specific genetic disorders may be constrained by the effect on brain development, levels of attainment may well be significantly altered depending on the individual's educational environment. 


\section{Acknowledgements}

J.W. and J.B. were supported by a grant from the Wellcome Trust with further support from the PWS Association and the Isaac Newton Trust. We would like to thank all those people with PWS and our control groups, their families, and other carers who helped us with this study.

\section{References}

Aman M.G., Singh N.N., Stewart A.W. \& Field C.J. (1986) Aberrant Behatior Checklist. Slosson, New York, NY.

Boer H. Holland A.J., Whittington J.E., Butler J.V., Webb T. \& Clarke D.J. (2002) Psychozic illness in people with Prader-Willi syndrome due to chromosome 15 maternal uniparental disony, Lanat 359, 135-6.

Butler J.V., Whittington J.E., Holland A.J., Webb T., Butler J.V., Clarke D.J. \& Boe: H. (2002) Prevalence of and risk factors for physical ill-health in people with Prader-Willi Syndrome. Developmental Medicine and Child Newrology 44, 248-55.

Curfs L.M.G. \& Fryns J.-P. (1992) Prader-Willi syndrome: a review with special attention to the cognitive and behavioral profile. Bitth Defects: Original Article Series 28, 99-104.

Curfs L.M.G., Wiegers A.M., Sonmers J.R.M., Borghgraef M. \& Fryns J.P. (1991) Strengths and weaknesses in the cognitive profile of youngsters with Prader-Willi syndrome. Clinical Genetics 40, 430-4.

Dykens E.M., Hodapp R.M. \& Finucane B.M. (2000) Genetics and Mental Retardation Syndrones Paul H Brookes, Baltimore, MD.

Dykens E.M., Hodapp R.M., Walsh K. \& Nash L J. (1992) Profiles, correlates, and trajectories of intelligence in Prader-Willi syndrome. American Academy of Child and Adolescent Psychio arry 31, 1125-9.

Dykens E.M. \& Kasari C. (1997) Maladaptive behavior in children with Prader-Willi syndrome, Down syndrome, and nonspecific Mental Retardation. American Joumal on Mestal Retardation 102, 228-37.

Einfeld S. \& Tonge B.J. (1989) Developmental Behariour Chedklist - Parental wersion Centre for Developmental Psychiatry, Clayton.

Hodapp R.M. \& Dykens E.M. (2001) Strengthening Behavioural Phenotype Research on Genetic Mental Retardation Syndromes. Amerian Journaton Mental Retardation 106, 4-15.

Holland A.J., Treasure J., Coskeran P., Dallow J., Milton N. \& Hillhouse E. (1993) Measurement of Excessive Appetite and Metabolic Changes in Prader-Willi Syndrome. Intematienal Joumal of Obesity 17, 526-32.

Holn V.A., Cassidy S.B., Butler M.G., Hanchett I.M., Greenswag L.R., Whitman B.Y. \& Greenberg F. (1993) Prader-Willi Syndrome: Consensus Diagnostic Criteria. Pediatrics 91 , $398-402$ 
Johns M. W. (1991) A new method for measuring daytime sleepiness: The Epworth Sleepiness Scale. Slep 14, 540-5.

Nicholls R.D. (1993) Genomic imprinting and candidate genes in Prader-Williand Angelman syndromes and mouse models. Cunem Opmon in Genetics and Development 3, 445 456

Prader A., Labhart A. \& Willi H. (1956) Ein Syndrom von Adipositas, Kleinwuchs, Kryptorchismus und Oligophrenie nach myatonieartigen Zustand im Neugeborenenalter. Solweizerische Medizmishe Wodhendhiff 86, 1260-1

Roof E., Stone W., MacLean W., Feurer I.D., Thompson T. \& Butler M.G. (2000) Intellectual characteristics of Prader-Willi syndrome: comparison of genetic subtypes joumal of Intellethal Disability Researh 44, 25-30.

Rust J. (1995) Wedsher Objective Language Dimensions (WOLD), Wechiler Objetive Numerical Dimensions (WOND). Psychological Corporation. Harcourt Brace, New York, NY.

Sparrow S.S., Balla D.A. \& Cicchetti D.V. (1984) Vincland Adaptive Behavior Siales. NFER-Nelson, Windsor.

Stauder J.E., Brinkman M.J. \& Curfs L.M. (2002) Multi-modal P3 deflation of event-related brain activity in Prader-Will syndrome. Newrocience Leters 327, 99-102.

Warren H.L. \& Hunt E. (1981) Cognitive processing in children with Prader-willi syndtome. In The Prader-Willi Syudrome. (eds V.A. Holm, S.J. Sulabacher \& P.L. Pipes), pp. 161-77. University Park Press, Baltimore, MD.

Wechsler D. (1993) The Wechsler Adut Intelligence Scale (WAIS-Revised UK Edition), Wrohster

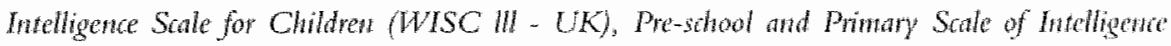
(WPPSI-Revised UK), Wedhler Objedive Reading Dimensions (WORD). Psychological Corporation. Harcourt Brace, New York, NY.

Whittington J.E., Holland A.J., Webb T., Butler J.V., Clarke D.J. \& Bocr H (2001) Population prevalence and estimated birth incidence and mortality rate for people with Prader-. Willi Syndrome in one UK Health Region Joumal of Medical Generics 38, 792-8.

Whittington J.E, Holland A J., Webb T., ButlerJ.V., Clarke D.J. \& Boer H. (2004) Cognitrwe abilities and genotype in a populacion-based sample of people with Prader-Will syndrome. Joumal of Inethectual Disobility Resamh 48, 172-87. 

Chapter $4 \mathrm{a}$

Problem behaviors in $5 p$ - Cri du chat syndrome, 15q- Prader-Willi syndrome and $17 p$ - Smith-Magenis syndrome.

\section{A comparative study using the Aberrant Behavior Checklist}

Published as:

Clarke* D.J. \& Boer* H. (1998) Problem behaviors in 5p-cri du chat syndrome, 15qPrader-Willi syndrome and $17 \mathrm{p}$-Smith-Magenis syndrome. A comparative study using the Aberrant Behavior Checklist. American Joumal on Mcytal Retadation 103, 264-71

*) Contributed equally to the study 


\section{Abstract}

Problem behaviom in individuals who had one of three chromosome deletion disorders (5p- Cri du chat syndrome [CDCS]; 15q-Prader-Willi syndrome [PWS]; and 17p-Smith-Magenis syndrome [SMS]) were investigated. The Aberrant Behavior Checklist was used. Results were contrasted with those of two groups of people with mental retardation who were reported in other studies. The checklist rates many, but not all, potentially relevant behaviors. Eating abnormalities, known to be problematic in Prader-Willi syndrome, and sleep abnormalities, believed to be problematic in Smith-Magenis syndrome, are not included in the survey.

All three chromosome deletion disorders are associated with greater ratings of severity of problem behaviors than the comparison groups on at least one sub-scale of the checklist. The results lend support to the partial specificity model of behaviors associated with genetically determined syndromes.

Much of the limited work on behavioral aspects of genetically determined syndromes consists of anecdotal descriptions of behaviour or has relied on the use of instruments for which there are no published norms or comparison samples. This does not allow an assessment of whether behaviors are characteristic of a syndrome, or merely occurring with a frequency typical of children or adults with mental retardation. Some studies have used standard assessment instruments, and contrast samples, producing more robust findings (e.g., Clarke, Boer, Chung, Sturmey \& Webb, 1996). Researchers are increasingly sceptical of clinical diagnoses of syndromes associated with genetic abnomalities, and believe that it is preferable to describe relatively pure samples of people with one type of genetic abnomality (eg "deletion" Prader-Willi syndrome or 15q-PWS, as distinct from "disony" Prader-Willi syndrome). The subjects with Prader-Willi syndrome in the present study all had $15 q$ - Prader-Willi syndrome (using methylation analysis at the SNRPN locus).

Hodapp (1997) has described the debate about the nature of behaviors associated with genetically determined syndromes, with investigators placing different cmphasis on their specificity. Some support a "no-specificity" position (that all genetic disonders are thought to have identical effects on behavior), and others, a "total specificity" position (that each genetic disorder has unique effects). Hodapp hypothesised that the partial specificity model (that a few genetic disorders share an identical behavior, or cluster of behaviors, not shared by mixed etiological groups) was likely to be applicable to most genetic syndromes. The debate is of clinical relevance, because behavioral items have been used in some diagnostic criteria for disorders such as Prader-Willi syndrome (eg Holm et al, 1993). In the present study our purpose was to contribute to this debate, and by detemining specific 
behaviors associated with the three syndromes, thereby assisting the search for effective interventions.

All subjects in the present study were reported to have chromosome deletions, with documentation of the person or laboratory establishing the genetic diagnoses. A well-researched and psychometrically robust behavioral checklist, the Aberrant Behavior Checklist (Aman, Singh, Stewart \& Field, 1985a, 1985b) was used to assess problem behaviors. Findings were compared to those of Aman $\&$ Singh (1986) who reported Aberrant Behavior Checklist sub-scale scores and standard deviations (SDs) for 688 people with mental retardation who resided in institutions (many with severe retardation) and with the results of Marshburn \& Aman (1992), who reported on 539 people with mental retardation aged 6 to 21 years who lived in community settings.

We examined the behavior of individuals with three syndromes: cri du chat, Prader-Willi, and Smitli-Magenis. Cri du chat syndrome affects about 1 in 50,000 live born infants (Udwin \& Dennis, 1995). Clinical features include prominent epicanthal folds, hypertelorism, posteriorly rotated low set ears, a broad nose and micrognathia. Hypotonia and growth retardation occur, and the associated intellectual disability is usually severe. The disorder results from loss of genetic material from $5 \mathrm{p}$. The loss is usually the result of a de novo deletion (in about $85 \%$ of cases), usually of paternal origin (Udwin \& Dennis, 1995). Deletion sizes vary, but the critical region for cri du chat syndrome is thought to be $5 \mathrm{p} 15.2$, with the infantile cat-like cry resulting from deletion of 15.3 (Gersh et al, 1995). Most other cases arise as a result of unbalanced translocations. Little is known about behavioral abnormalities associated with the disorder. Cornish \& Pigram (1996) described 27 children with cri du chat syndrome. Using a descriptive questionnaire they noted the following behaviors: self-injury, repetitive movements, hypersensitivity to sound, clumsiness and obsessive attachments.

Prader-Willi syndrome (PWS) affects about 1 in 15,000 infants, resulting in hypotonia and feeding difficulty. Many infants are tube-fed. In childhood there is a switch to pathological over-eating. Affected adults are of short stature, with hypogonadism, small hands and feet, and a relatively characteristic pattern of facial dysmorphology. PWS results from the absence of a paternal contribution to $15 \mathrm{q} 11-13$, arising from a paternal deletion (about $70 \%$ of cases) or maternal uniparental disomy (about $30 \%$ of cases). A small number of affected people may have an imprinting error. PWS is usually associated with mild or borderline mental retardation. Apart from the eating disorder, a number of other problem behaviors have been reported in association with the syndrome, including outbursts of temper, sleep abnormalities, seli-injury through skin picking, repetitive speech or persistent questioning (Clarke, Boer \& Webb, 1995; Clarke et al, 1996) and obsessional behaviors (Dykens, Leckman \& Cassidy, 1996). 
Smith-Magenis syndrome (SMS) results from a deletion 17p11.2. It is a rare disorder, with an incidence estimated at around 1 in 50,000 live born infants (Colley, Leversha, Voullaite \& Rogers, 1990). The physical characteristics associated with the syndrome are variable, and may include abnomal head shape (brachycephaly), and broad nasal bridge, flat mid-face, "cupid"s bow" shaped upper lip, abnormal ear shape and position, incurving fingers, and small toes. The voice is often hoarse or deep (Greenberg et $\mathrm{l}_{\mathrm{l}}$ 1991). Eye abnormalities include squint, short-sight and detachment of the retina. Finucane, Jaeger, Kurtz, Weinstein \& Scott (1993) suggested that people with Smith-Magenis syndrome may be prone to retinal detachment because of a combination of short-sightedness, overactivity, and self-injurious behaviors including head-banging. Udwin and Dennis (1995) said that Smith-Magenis syndrome is "notable in that forid behavioural manifestations may precede and overshadow the learning disability and other features" (p.187). Colley et al, (1990) described a number of unusual behaviors, including a "self-hug" of the upper body, and sleep abnormalities.

\section{Method}

\section{Instrument}

Caregivers of people with cri du chat syndrome, Prader-Willi syndrome (the subjects with Prader-Willi syndrome in the study all had 15q- Prader-Willi syndrome, and Smith-Magenis syndrome were asked to complete the Aberrant Behavior Checklist, a 58 item checklist of maladaptive behaviors rated on a 4-point scale from 0 (not at all a problem) to 3 (a severe probleml. Results are stummarized in fuve subscale or factor scores: Factor I (Irritability, Agitation); Lethargy, Factor II (Social Withdrawal); Factor III (Stereotypic Behavior); Factor IV (Hyperactivity, Non-Compliance); and Factor V (Inappropriate Speech). The Aberrant Behavior Checklist, which has been used in a number of setrings, and with a variety of client groups (Aman, Burrow \& Wolford, 1995; Aman, Richmond, Stewant, Bell \& Kissel, 1987; Aman, Singh, Stewart \& Field, 1985a, 1985b; Freund \& Reiss, 1991; Marshburn \& Aman, 1992) has been found to be psychometrically robust. The original factor (subscale) structure has been replicated in comtries including the United States and the United Kingdom (Bihm \& Poindexter, 1991; Newton \& Stumey, 1988). The original Aberrant Behavior Checklist sub-scale structure has also been found to be valid for the modified version for use in commmunity settings (the Aberrant Behavior Checklist-C) (Aman et al, 1995). This was the fom of the checklist we used in the present study. Details of reliability and validity studies can be found in the ABC manual (Aman \& Singh, 1986). 
Figure 1: Diagrammatic representation of constellations of problem behaviors and syndromes.

\begin{tabular}{|c|c|c|c|c|}
\hline & ] & Inactiv & & \\
\hline & ] & $\begin{array}{l}\text { Repetit } \\
\text { speech }\end{array}$ & & \\
\hline $15 q-$ & ] & Labile & & {[} \\
\hline PWS & ] & Inabili & gratification & {[} \\
\hline & ]\} & Dyscon & temper, etc) & {$[17 p$} \\
\hline & ]) & Opposi & bedient,etc) & [ SMS \\
\hline $5 p-$ & ) & Imputs & & \\
\hline $\operatorname{coCs}$ & Y Di & tibility & odd, bizarre behaviors & [ \\
\hline & ) $R$ & ssness & & \\
\hline & Io & tivity & & \\
\hline
\end{tabular}

Subjects

People with the three syndromes were recruited for the study by contacting the relevant parent and carerorganizations and support groups (see Acknowledgements). With the exception of 1 nurse and 5 key workers, who rated 6 persons with Prader-Willi syndrome resident in community group homes, all ratings were provided by parents and were related to people living with their families. Of the responses obtained, 5 subjects with cri du chat syndrome, 19 with Prader-Willi syndrome and 5 with Smith - Magenis syndrome were excluded from the analysis because they were below the age of 5 years or because they did not have a chromosome deletion identified by a named clinician or unit. The questions used to ascertam the genetic abnormality were similar for all syndromes, and were worded as follows:

- Does (s)he have a chromosome abnormality (respondent to circle yes, no, or unknown)

- If yes, please circle, and give details below (respondent to circle type of genetic abnormality)

- Please write in the exact genetic abnormality, if you know it, and the person and place where it was found (headings were provided for respondent to give details of the person and place where the genetic diagnosis was established). The larger proportion of people with Prader-Willi syndrome excluded from the analyses reflects the relatively high proportion of people with uniparental disomy associated with this disorder. 
Table 1 . Characteristics of syndrome groups

\begin{tabular}{|c|c|c|c|}
\hline & \multicolumn{3}{|c|}{ Syndrome group } \\
\hline & $5 p-\operatorname{Cocs}(N-38)$ & $15 q-P W 5(N=55)$ & $17 \mathrm{p}-5 \mathrm{MS}(\mathrm{N}=21)$ \\
\hline Mern age (yrs) & 17.3 & 25.1 & 14.5 \\
\hline Age range (yrs) & $5-40$ & $6-43.5$ & $5.5-32.5$ \\
\hline \multicolumn{4}{|l|}{ Mean Body Mass } \\
\hline Iridex $\left(\mathrm{kgm}^{-2}\right)$ & 19.00 & 37.98 & 25.10 \\
\hline \multicolumn{4}{|l|}{ Mean ABC } \\
\hline \multicolumn{4}{|c|}{ Factor 5 cores $\left(5 . d_{n}\right)$} \\
\hline 1 & $13.21(9.05)$ & $14.59(11.90)$ & $21.67(11.15)$ \\
\hline II & $5.24(6.85)$ & $6.71(7.59)$ & $7.47(5.29)$ \\
\hline III & $5.29(5.25)$ & $1.55(2.49)$ & $7.00(4.55)$ \\
\hline IV & $21.68(10.88)$ & $7.60(6.27)$ & $26.10(10.13)$ \\
\hline V & $2.39(3.48)$ & $4.20(3.44)$ & $6.10(3.66)$ \\
\hline$M: F$ & $15: 23$ & $28: 27$ & $9: 12$ \\
\hline \multicolumn{4}{|c|}{$\begin{array}{l}\text { Severity of retardation } \\
\text { (as reported to carers) }\end{array}$} \\
\hline & $(\mathrm{N}=32)$ & $(\mathbb{N}=50)$ & $(N=19)$ \\
\hline None & 0 & 2 & 0 \\
\hline Mitid & 1 & 21 & 2 \\
\hline Moderate & 4 & 24 & 12 \\
\hline Severe & 24 & 3 & 5 \\
\hline Profound & 3 & 0 & 0 \\
\hline
\end{tabular}

Other information

Caregivers also gave information regarding date of birth, height, weight, gender, and severity of mental retardation (as reported to caregivers by local services usually educational or clinical psychologists). We did not administer intelligence tests. Parents were given the opportunity to telephone one of the authors if they had questions about the study, or if they were unsure about how to complete the questionnaire.

\section{Results}

There were 15 males and 23 fenales with cri du-chat syndrome, 28 males and 27 females with Prader-Willi syndrome, and 9 males and 12 females with Smith-Magenis syndrome in our sample. Their mean ages (with ranges in parentheses) respectively, were 17.3 ( 5 to 40$), 25.1$ (6 to 43.5 ), and 14.5 (5.5 to 32.5 ) years. The mean Body Mass Index-a measure of weight corrected for height 
Table 2. Comparison of z scores for mean $A B C$ factors in three deletion disorders, using Arran \& Singh (1986) contrast group* as standardization sample.

\begin{tabular}{llllll}
\hline & ABC factor & & & \\
& I & II & III & IV & V \\
\hline $5 p-\operatorname{CDCS}(N=38)$ & 0.503 & -0.393 & -0.034 & 1.011 & 0.346 \\
$15 q-$ PWS $(N=55)$ & 0.656 & -0.238 & -0.638 & -0.324 & 1.081 \\
$17 p-$ SMS $(N=21)$ & 1.437 & -0.159 & 0.342 & 1.431 & 1.854 \\
\hline
\end{tabular}

* 688 persons with mental retardation aged $5-50$ years and resident in institutions.

Table 3. Comparison of $z$ scores for mean ABC factors in three deletion disorders, using Marshburn \& Aman (1992) contrast group* as standardization sample.

\begin{tabular}{llllll} 
& \multicolumn{2}{l}{ ABC factor } & & & \\
& I & II & III & IV & $V$ \\
\hline $5 p-\operatorname{COCS}(N=26)$ & 0.612 & 0.026 & 0.744 & 0.807 & -0.103 \\
$15 q-$ PWS $(N=19)$ & 1.079 & 0.208 & -0.162 & -0.114 & 1.406 \\
$17 p-$ SMS $(N=18)$ & 1.633 & 0.110 & 1.229 & 1.063 & 1.782 \\
\hline
\end{tabular}

calculated by dividing weight by height squared-was 19.00 for subjects with cri du chat, 37.98 for those with Prader-Willi, and 25.10 for individuals with Smith-Magenis syndrome. Table 1 provides subjects' scores on the Aberrant Behavior Checklist, and Table 2 shows level of mental retardation by syndrome. The results are consistent with existing knowledge regarding physical aspects of the syndromes (e.g., that many adults with Prader-Willi syndrome are overweight for height and that cri du chat syndrome is associated with growth retardation and low body weight). Aberrant Behavior Checklist subscale scores were compared by gender, and no significant differences were found. Combined information for both genders is presented in the tables. Relations between age and Aberrant Behavior Checklist subscale scores and between Body Mass Index and Aberrant Behavior Checklist subscale scores were assessed by calculating Pearson correlation coefficients, with two-tailed testing of significance. The only significant correlations were between age and Factor V (Inappropriate Speech) scores in 5pcri du chat syndrome, $\mathrm{r}=.534, \mathrm{p}<.01$, and between Body Mass Index and Factor I (Irritability, Agitation), $r=.305, p<.05$, and Factor II (Lethargy, Social Withdrawal), $r=.306, p<.05$, in 15q-Prader-Willi syndrome.

First Comparison Group

We compared our sample with 688 institutionalized subjects studied by Aman and Singh (1986). Table 3 details $z$ scores (number of SDs separating the means) for the 
three deletion syndromes compared to the mean Aberrant Behavior Checklist subscale scores reported by Aman and Singh (1986) in the Aberrant Behavior Checklist manual. Aman and Singh's sample contained individuals aged 5 to $51+$ who lived in institutions and predominantly had severe or profound mentall retardation. To ensure comparable ages in the two groups (deletion syndromes and Aman and Singh's population), we combined the age ranges between 5 and 50 reported by Aman and Singh and excluded the $51+$ age band. This comparison is probably most appropriate for the subjects with cri cu chat syndrome, which is usually associated with severe mental retardation.

\section{Second Comparison Group}

Table 3 also shows z scores for the comparison between the Aberrant Behavior Checklist subscale means for the three deletion syndromes and 539 people with mental retardation who were between 6 and 21 years of age and lived in community settings (Marshburn \& Aman, 1992). Almost all subjects with deletion syndromes resided with their families or in community group homes, so this comparison is probably more appropriate for the $15 q$-Prader-Willi syndrome and $17 \mathrm{p}-$ Smith-Magenis syndrome groups.

\section{Most Problematic Behaviors}

Table 4 shows the 10 Aberrant Behavior Checklist items that had the highest scores for each deletion syndrome group, reinforcing the information in Table 3 and providing a clinically meaningful description of the most problematic behaviors.

Behavior in cri du chat syndrome was characterized by overactivity, restlessness, impulsiveness, inattentiveness, and distractibility. Prader-Willi syndrome was characterized by temper outbursts, inability to delay gratification, repetitive speech, lability of mood, inactivity, and self-injury. Smith-Magenis syndrome was associated with impulsiveness, temper outbursts, distractibility, labile mood, and bizarre and boisterous behaviors. The most problematic behaviors among individuals with Smith-Magenis and Prader-Willi syndromes were similar (relating to impulsiveness and mood instability), whereas those in cri du chat syndrome were somewhat different (being centered on restlessness and distractibility). Distractibility was more of a problem for individuals with Smith-Magenis syndrome, and inactivity more problematic for those with Prader- Willi syndrome. 


\section{Discussion}

This study is one of the first in which (a) a standard behavior assessment was used to rate behaviors in samples of people with chromosome deletion disorders (rather than clinically defined syndromes) and (b) contrast groups of people with mental retardation living in community and institutional settings reponted by other authors were utilized for comparison.

The results relating to Prader-Willi syndrome are largely consistent with previous research using other methodologies (e.g., Clarke et al., 1996; Clarke, Waters, \& Corbett, 1989; Dykens, Hodapp, Walsh, \& Nash, 1992). Our results are consistent with those of Colley et al. (1990), one of the few studies of behavior of individuals with Smith-Magenis syndrome. Our results relating to cri du chat syndrome suggest greater problems with overactivity and restlessness than were found by Cornish and Pigram (1996). These authors used the Society for the Study of Behavioral Phenotypes Postal Questionnaire (0'Brien, 1995) to assess developmental and behavioral characteristics of 27 children with cri du chat syndrome, 6 of whom were rated as underactive and 2 as overactive. This questionnaine, which includes develop mental and behavioral items, has not been used as widely as the Aberrant Behavior Checklist, and less is known about its psychometric properties. Firm genetic diagnoses, the use of multiple items relating to overactivity in the Aberant Behavior Checklist, and the use of comparison groups add weight to our findings. Results with regard to other problem behaviors were consistent between the studies. Of Cornish and Pigram's sample of 27 individuals with cri du chat syndrome, 19 had repetitive movements, 10 were "irritable," and 9 had serious temper tantrums.

The present study has a number of methodological weaknesses. Because of our source of subjects (resulting from a survey of support organizations tor each of the three syndromes), there is likely to be ascertainment bias towards tesponders with relatively high social status as well as bias due to use of questionnare methodology. Our sample size was relatively stmall (although larger than those in many previously reported studies). Further, the Aberrant Behavior Checklist is most reliable when completed by nurses or other caregivers who have previous traming on the instrument, although the checklist has been used extensively in the way in which it was employed in this study. Comparisons were made with samples from other countries, although experience and the literature suggest that Aberrant Behavior Checklist items and the factor structure of this instrument are applicable in the United Kingdom, the United States, and New Zealand. Aberrant Behavior Checklist item scores have been reported, as have subscale scores, because the Aberrant Behavior Checklist factor structure combines some item scores in a way that may mask differences between syndrome groups. For example, both clinical experience and results of the present study suggest that Prader-Willi syndrome is 
associated with lethargy, but not social withdrawal (both are combined into Aberrant Behavior Checklist Factor 11, Social Withdrawal). Given the rarity of the disorders surveyed, the lack of other published literature relating to problem behaviors in which investigators employed standardized instruments and comparison groups, and the difficulties intherent in using other methodologies (e.g., direct observation), we belicve that the present results will be of interest to clinicians and researchers. In our study we deliberately focused on problem behaviors because these often have a major impact on quality of life for affected people and their caregivers. It is not our intention to suggest that behavior of individuals with any of the disorders described here is characterized mainly by problems. We are aware that many caregivers describe positive personality characteristics associated with such disorders.

A standardized instrument such as the Aberrant Behavior Checklist can only be used to assess a limited number of defined problem behaviors. It therefore cannot be employed to demotastrate some differences relating to other behaviors that may be very problematic (e.g., the eating disorder associated with Prader-Willi syndrome or sleep abnormalities in individuals with Smith-Magenis syndrome). For this reason, our results should not be taken as an exhaustive list of problem behaviors associated with the three syndromes but, rather, as a preliminary exploration of relative vulnerabilities. Our hope is that a knowledge of such vulnerabilities, combined with knowledge about genetic abnomalities, will allow the impact of environmental factors relevant to treatment to be assessed. For cxample, Prader-Willi syndrome has now been shown to be associated with temper outbursts to a much greater degree than is usual among other samples of people with mental retardation. However, not all people with Prader-Willi syndrome have such a problem, and the severity of tantrums may vary over time. Further study of environmental modifiers may lead to useful interventions, and, conversely, a knowledge of the gene product(s) in Prader-Willi syndrome nay facilitate research into impulsive behaviors.

These results support the partial specificity model of behaviors associated with genetically deternined disabling disorders, suggesting that each deletion disorder is associated with a relatively characteristic constellation of problem behaviors, but with some of the behaviors occurring in association with more than one disorder (see Table 5 ).

Some of the people with chromosome deletion disorders described in the present study have, or will be, described in other reports. These include 21 adults with Prader-Willi syndrome reported in Clarke et al. (1996) and 38 people with $5 \mathrm{p}$-cri du chat syndrome who will be described in a United Kingdom/United States collabonative study conducted by Dykens and Clarke (in press). 
Note

Some of the people with chromosome deletion disorders reported here have, or will be, described in other reports. These include 21 adults with $15 q-$ PWS reported in Clarke, Boer, Webb, Chung \& Sturmey (1996) and 38 people with $5 \mathrm{p}-\mathrm{CDCS}$ to be reported as part of a UK/US collaborative study.

\section{Acknowledgements}

The authors are grateful for the help of the Cri du Chat Syndrome Support Group, the Prader-Willi Syndrome Association (UK) and the Smith Magenis Syndrome Contact Group.

\section{References}

Aman M.G., Burrow W.H., \& Wolford P.L. (1995) The Aberrant Behavior Checklist Conmunity: Factor validity and effect of subject variables for adults in group homes. American Joumal on Mental Retardation 100, 283-92.

Aman M.G., Richmond G., Stewart A.W., Bell J.C. \& Kissel R.C. (1987) The Aberrant Behavior Checklist: Factor structure and effect of subject variables in American and New Zealand facilities. American Joumal of Mental Deficiency 91, 570-8.

Aman M.G. \& Singh N.N. (1986) Aberrant Behavior Checklist Manual. Slosson Educational Publications, New York

Aman M.G., Singh N.N., Stewart A.W. \& Field C.J. (1985a) The Aberrant Behavior Checklist: A behavior rating scale for the assessment of treatment effects. American Joumal of Mental Deficiency $91,485-91$.

Aman M.G., Singh N.N., Stewart A.W. \& Field C.J. (1985b) Psychometric characteristics of the Aberrant Behavior Checklist. Amcrian Jountal of Mental Deficioncy 89, 492-502.

Bihm E.M. \& Poindexter A.R. (1991) Cross-validation of the factor structure of the Abermant Behavior Checkdist for persons with mental retardation. American Jouryat on Mental Retardarion $96,209-11$.

Clarke D.J., Boer H., Chung M.C., Sturmey P. \& Webb T. (1996) Maladaptive behaviour in Prader-Willi syndrome in adult life. Joumal of Indellectual Disability Rescarth 40, 159-65.

Clarke D.J., Boer H. \& Webb T. (1995) Genetic and behavioural aspects of Prader-Willi syndrome: a review with a translation of the original paper. Mental Handicap Research 8 , $38-53$.

Clarke D.J., Waters J, Corbett J.A. (1989) Adults with Prader-Will syndrome: abnomalities of sleep and behaviour. Journal of the Royal Society' of Mediain 82, 21-4.

Colley A.F., Leversha M.A., Voullaire L.E. \& Rogers J.G. (1990) Five catses demonstrating the distinctive behavioural features of chromosome deletion 17(p11.2p11.2) (Smith-Magenis syndrome) Journal of Paediatrics and Child Health 26, 17-21. 
Cormish K.M. \& Pigram J. (1996) Developmental and behavioural characteristics of cri du chat syndrome. Arhines of Discase in Childhood 75, 448-50.

Drykens E.M., Hodapp R.M., Walsh K.K. \& Mash L. (1992) Adaptive and maladaptive behavior in Prader-Will Syndrome. Joumal of the American Academy of Child and Adolecent Psydiatry 31, 1131-6.

Dykens. E.M., Leckman J.F. \& Cassidy S.B. (1996) Obsessions and compulisions in. Prader-Willi syndrome. Jownal of Child Psychology and Psychiatry 37, 995-1002.

Finucane B.M. Jaeger E.R., Kurtz M.B., Weinstein M. \& Scott C.I. (1993) Eye abnormalities in the Smith-Magenis contiguous gene syndrome. American Journal of Medial Geretis 45 , $443-6$.

Freund L.S. \& Reiss A.L. (1991) Rating problem behaviors in outpatients with mental retardation: Use of the Aberrant Behavior Checklist. Researd in Dewelopmental Disabilities 12, $435-51$.

Gersh M. Goodart S.A., Pasztor L.M., Harris D.J., Weiss L. \& Overhauser J. (1995) Evidence for a distinct region causing a cat-like cry in patient with $5 p$ deletions. Amerion Jowmal of Homan Genetics 56, 1404-1410.

Grcenberg F., Guzzeta V., Montes de Oca Luna R., Magenis R.E., Smith A.C.M., Richter S.F., Kondo I., Dobyns W.B., Patel P.I. \& Lupski J.R. (1991) Molecular analysis of the Smith-Magenis syndrome: a possible contiguous-gene syndrome associated with del(17)(p11.2). American Jow thal of Hwhan Gentios 49, 1207-18.

Hodapp R.M. (1997) Direct and indirect behavioral effects of different genetic disorders of mental retardation. American Joumal on Mental Retardation 102, 67-79.

Holn V.A., Cassidy S.B., Butler M.G.. Hanchett J.M., Greenswag L.R., Whitman B.Y. \& Greenberg F. (1993) Prader-Willi syndrome: consensus diagnostic criteria. Pediatrics 91 , $398-402$.

Marsthiom E.C. \& Aman M.G. (1992) Factor validity and noms for the Aberant Behavion Checklist in a community sample of children with mental retardation. Jownal of A wism and Developunewal Disorders $22,357-73$.

Newton J. T. \& Stumey P. (1988) The Aberrant Behavior Checklist: A British rephication and extemsion of psychometric properties. Jounal of Mental Deficiency Researd 32, 87-92.

Udwin O \& Dennis J. (1995) Psychological and behavioural phenotypes in genetically determined syndromex a review of research findings. In: Beharoural Phenotypes (eds G. O'Brien \& W. Yule). Mackeith Press, London. 
Chapter $4 b$

\section{Development and behaviour in two genetic syndromes:}

\section{1: Prader-Willi syndrome}

Published as:

Boer H. \& Clarke D. (1999) Development and Behaviour in Two Genetic Syndromes: 1: Prader-Willi Syndrome. Journal of Applied Research in Intellectual Disabilities 12 , 294-301. 


\section{Summary}

Development and behaviour was investigated in samples of 210 children and adults with Prader-Willi syndrome (PWS) using the Society for the Study of Behavioural Phenotypes Postal Questionnaire. There were few differences between males and females. Children and adults were compared. Most adults with PWS had good self-care skills and good use of speech. Most adults walked without problems but many needed help to dress. PWS adults had more problems than children with daytime sleepiness and other sleep problems, unusual routines, and self-injury; and fewer problems with faddy eating, verbal abuse and stubborness. Changeable mood was a problem for both adults and children, adults had more problems with low mood.

\section{Introduction}

Prader-Willi syndrome (PWS) (Prader et al. 1956) is characterised by different clinical features at various stages of life. Feeding difficulties, often requiring tube feeding, are apparent in the neonatal period, as is marked hypotonia. In childhood, there is a switch to over-eating, now known to be due to delayed and incomplete satiation of appetite (Holland et al. 1995). Affected adults are of short stature, with a characteristic facial appearance, small hands and feet, and hypogonadism. The eating disorder leads to obesity if not managed by dietary restriction. PWS is associated with deletions of paternal origin at $15 q 11-13$ in about $70 \%$ of cases, and with maternal uniparental disomy in most of the remaining cases. The gene(s) affected in Prader-Willi syndrome are believed to be subject to genomic imprinting (Dittrich et al. 1992).

A number of behavioural abnomalities have been described in association with PWS, including outbursts of temper, mood instability, self-injury, impulsiveness, repetitive speech, obsessional symptoms (Dykens et al. 1992; 1996; Clarke et al. 1996) and sleep abnomalities (Clarke er al. 1989; Harris \& Allen, 1996) in addition to the eating disorder (Holland of al. 1993). Psychoses have also been described (Kollack \& Wolff, 1966; Clarke et al 1998).

The recognition of behavioural profiles is of increasing interest and importance. Problem behaviours, or abnormal emotional states, are usually cited by carers as being more difficult to cope with than physical medical problems. Recognition of characteristic patterns of maladaptive behaviour, or emotional problems, is also the first step towards developing effective treatments or management strategies. Recont years have seen an increase in studies on the behavioural aspects of genetic disorders and there is a growing recognition that a number of syndromes have behavioural features as a prominent component of their phenotype (O'Brien and 
Yule, 1995). However, the relation between genetic abnormalities and phenotypic expression of emotional or behavioural disorders is likely to be complex, and may result from intervening or complicating variables rather than directly from the genetic abnormality. As in any other sample of humans, there is individual variation among people with genetic disorders, and the results from studies of large samples should not be taken as an infallible guide to predict the behaviour of individuals.

Hodapp (1997) has described three models of the behavioural effects of genetically determined disabling syndromes: the "no-specific effect" model in which all genetic syndromes are believed to lead to identical or very similar behaviours; the "total-specificity" model in which each disorder is believed to lead to a unique pattern of behaviour, and the "partial-specificity" model in which two or more syndromes lead to similar patterns of behaviour that may differ from those observed among other individuals with intellectual disabilities. Use of the same instrument to assess developmental and behavioural aspects of genetically determined disorders allows comparisons to be made between syndromes and over time. The Society for the Sudy of Behavioural Phenotypes Postal Questionnaire, used in this study, has recently been used to describe behaviour among children with Cri-du-chat syndrome (Cornish \& Pigram, 1996). The SSBP-PQ was designed specifically to allow developmental and behavioural aspects of genetic disorders to be compared between syndromes.

The present study describes developmental and behavioural aspects of PWS in a manner that is clinically informative. The influence of age and gender on behaviour and development are addressed.

In the following paper, Boer et al. (1999) describe development and behaviour in Rubinstein-Taybi syndrome using the same instrument.

\section{Method}

Members of the Prader-Willi Syndrome Association (UK) were asked to complete the Society for the Study of Behavioural Phenotypes Postal Questionnaire (SSBP..PQ). Members of this association are almost exclusively relatives (usually parents) of children and adults with the syndrome.

The SSBP-PQ, a 98 question structured interview with 8 developmental domains and 10 behavioural domains, was piloted with the help of the support group for parents and carers of people with agenesis of the corpus callosum ("Corpal"). The questionnaire was circulated to 70 members of Corpal on two occasions (to allow test-retest reliability to be measured), and response rates of $67 \%$ and $64 \%$ obtained for the two mailings (O'Brien, 1994). Statistical analysis examined test-retest reliability using Kappa and weighted Kappa for each item. Reliability was found to be 
Table 1. Test re-test reliability data for $5 S B P-P Q^{*}$

\begin{tabular}{|c|c|c|c|c|}
\hline \multirow{2}{*}{$\begin{array}{l}\text { Behavioural domain } \\
\text { Sleep }\end{array}$} & \multirow[t]{2}{*}{ Kappa } & \multicolumn{3}{|c|}{$95 \%$ confidence limits } \\
\hline & & & & \\
\hline Regular pattern & 0.3428 & 0.096 & to & 0.7801 \\
\hline Settling difficulty & 0.2044 & 0.2818 & to & 0.6906 \\
\hline \multicolumn{5}{|l|}{ Social behaviour } \\
\hline $\begin{array}{l}\text { Emotional communicativeness } \\
\text { Movement and activity }\end{array}$ & 0.3136 & 0.0748 & to & 0.7019 \\
\hline Time on task & 0.2105 & 0.0104 & to & 0.4313 \\
\hline Wweighted & 0.3601 & 0.1489 & to & $0.5714]$ \\
\hline Creates chaos aimlessly & 0.4000 & 0.0383 & to & 0.8383 \\
\hline Other problems & 0.1529 & 0.1564 & to & 0.4623 \\
\hline \multicolumn{5}{|l|}{ Self-injurious and aggressive behaviours } \\
\hline Verbal abuse & 0.4776 & 0.2254 & to & 1.0000 \\
\hline [weighted & 0.6500 & 0.1790 & to & $1.0000]$ \\
\hline Other problems & 0.1304 & -0.3276 & to & 0.5885 \\
\hline \multicolumn{5}{|l|}{ Anxiety and mood } \\
\hline Other problems & 0.1053 & -0.4854 & to & 0.6959 \\
\hline
\end{tabular}

* data for least reliable items, from 0'Brien (1994)

high with the exception of nine items (detailed in Table 1), three of which related to "other problems" within behavioural domains, which inevitably have low repeat reliability. The questionnaire was acceptable to, and easily comprehended by, parents, who reported few problems in interpreting or responding to items. The discriminatory power of the questionnaire was also assessed, by examining the correlation between behavioural and developmental abnormalities and the presence of partial or total agenesis of the corpus callosum, and found be high. The behavioural abnormalities reported were comparable with those suggested in previous research (O'Brien, 1995).

The final version of the SSBP-PQ allows developmental and behavioural aspects of genetic disorders to be compared between syndromes. It consists of sections dealing with: prenatal and perinatal conditions, neurological problems, development (including assessments of vision, hearing, mobility, motor dexterity, feeding, continence, dressing, hygiene, and communication) and behavioural abnormalities. The latter include feeding, sleep, social behaviour, activity, communication, movements, interests, aggressive behaviours, self-injury, irritability, temper tantrums, and mood abnormalities. The development and characteristics of the SSBP-PQ have been described more fully elsewhere (Cornish \& Pigram, 1996; O'Brien, 1994; O'Brien, 1995; Clarke, 1995). 


\section{Results}

Three hundred and sixty questionnaires were mailed to the Prader-Willi Syndrome Association (UK), and 226 replies received, in response rate of $63 \%$. Sixteen responses were excluded from the analysis presented here; 3 because the diagnosis was in question, 2 because important information (such as date of birth, or sex) was not given, and 11 replies concerning children below the age of 3 years. The mean age of males was 17.1 years (range 3 to 51 years) and of females 16.4 years (range 3 to 49 years). The mean body mass index (weight in kilograms divided by the square of height in meters) of males was 30.8 and of females 28.7 . The mean body mass index of the group aged $3-16$ years (mean age 9.6 years) was 23.8 and 34.0 for the group aged over 16 years.

Other results are summarised in Tables 2 and 3 . A fornat similar to that employed by Cornish and Pigran in their study of Cri du chat syndrome has been used to tabulate results. Table 2 summarises items relating to development, comparing people between the ages of 3 and 16 years with those aged 17 years and over. Table 3 stimmarises items relating to behaviour, giving results of children and adults as well as the totals. The items relating to the amount of food eaten, and excessive daytime sleepiness, probably underestimate the problems associated with PWS. Many carers interpreted "excessive" daytime sleepiness to mean prolonged periods of sleep during the day, and commented in a free text section of the questionnaire that the person with PWS fell asleep easily when travelling by car, or in other low-arousal environments. Many carers also commented that the person with PWS ate less than normal because he or she was dieting, but would eat large amounts if not supervised.

One child with PWS had a hearing aid. Most adults with PWS had good self-care skills and communicated using speech. Over half of the PWS adults walked without problems. PWS was associated with eating abnormalities, sleep problems, under-activity, repetitive speech, verbal abuse, self-injury and nood abnomality. Most of the differences in the developmental domains between children and adults with $\mathbb{P}$ WS were consistent with skill acquisition, although longitudinal studies would be necessary to confirm this. PWS adults had more problems than children with daytime sleepiness and other sleep problems, unusual routines, and self-injury; and fewer problems with faddy eating, verbal abuse and stubbornness. Changeable mood was a problem for both age groups in the PWS sample, children tending to be excessively happy and adults excessively unhappy.

There were few differences between males and females, but "Problems shopping", "Creates chaos aimlessly" "Deliberately destroys things" and some of the items relating to mood abnomality seemed more problematic for males than females. 
Table 2. Comparison of children (3-16 years) and adults (17-51 years) with PWS: development.

\begin{tabular}{|c|c|c|c|c|c|}
\hline Item & $3-16$ years & $17-51$ years & Total & $x^{2}$ & $\mathrm{p}$ \\
\hline \multicolumn{6}{|l|}{ Epilepsy } \\
\hline Seizure(s) in past year & $9 / 117$ & $4 / 93$ & $13 / 210$ & 1.026 & NS \\
\hline Medication for epilepsy & $9 / 113$ & $4 / 91$ & $13 / 204$ & 1.076 & NS \\
\hline Seizure(s) before past year & $17 / 114$ & $16 / 90$ & $33 / 204$ & 0.305 & NS \\
\hline \multicolumn{6}{|l|}{ Vision and hearing } \\
\hline Wears glasses & $37 / 117$ & $44 / 93$ & $81 / 210$ & 5.382 & $<0.05$ \\
\hline Wears hearing aid & $1 / 114$ & $0 / 91$ & $1 / 205$ & NA & - \\
\hline \multicolumn{6}{|l|}{ Mobility } \\
\hline Can sit without support & $110 / 116$ & $86 / 86$ & $196 / 202$ & 4.584 & $<0.05$ \\
\hline Walks steadily and normally & $42 / 114$ & $48 / 87$ & $90 / 201$ & 6.705 & $<0.02$ \\
\hline Walks, not as steadily as others & $38 / 83$ & $21 / 55$ & $59 / 138$ & 0.781 & NS \\
\hline Walks, activity limited & $16 / 83$ & $15 / 55$ & $31 / 138$ & 1.214 & NS \\
\hline No independent walking & $10 / 83$ & $0 / 55$ & $10 / 138$ & NA & $\sim$ \\
\hline \multicolumn{6}{|l|}{ Dexterity } \\
\hline Gross hand movements & $111 / 115$ & $90 / 90$ & $201 / 205$ & NA & - \\
\hline Fine hand movements. & $87 / 112$ & $87 / 90$ & $174 / 202$ & 15.068 & $<0.001$ \\
\hline \multicolumn{6}{|l|}{ Feeding } \\
\hline Mot at all & $3 / 114$ & $0 / 89$ & $3 / 203$ & NA & - \\
\hline With fingers & $7 / 114$ & $0 / 89$ & $7 / 203$ & NA & - \\
\hline With spoon & $42 / 114$ & $0 / 89$ & $42 / 203$ & 41.343 & $<0.001$ \\
\hline With knife and fork & $62 / 1114$ & $89 / 89$ & $151 / 203$ & 54.577 & $<0.001$ \\
\hline Drinks from ordinary cup & $104 / 114$ & $90 / 91$ & $194 / 205$ & NA & - \\
\hline \multicolumn{6}{|l|}{ Continence and hygiene } \\
\hline Toilet trained & $57 / 115$ & $84 / 91$ & $141 / 206$ & 42.973 & $<0.001$ \\
\hline Washes normally & $63 / 113$ & $87 / 91$ & $150 / 204$ & 41.131 & $<0.001$ \\
\hline \multicolumn{6}{|l|}{ Dressing } \\
\hline \multicolumn{6}{|l|}{ Can dress self: } \\
\hline Completely & $30 / 111$ & $64 / 91$ & $94 / 203$ & 37.687 & $<0.001$ \\
\hline With little help & $40 / 111$ & $24 / 91$ & $64 / 203$ & 2.157 & NS \\
\hline With a lot of he.p & $15 / 111$ & $3 / 91$ & $18 / 203$ & 6.431 & $<0.02$ \\
\hline Not at all & $26 / 111$ & $0 / 91$ & $26 / 203$ & 24.464 & $<0.001$ \\
\hline \multicolumn{6}{|l|}{ Communication } \\
\hline Does not indicate needs & $2 / 110$ & $0 / 90$ & $2 / 200$ & NA & * \\
\hline Uses non-verbal method & $1 / 110$ & $2 / 90$ & $3 / 200$ & $N A$ & - \\
\hline Uses formal signs or symbols & $11 / 110$ & $0 / 90$ & $11 / 200$ & NA & - \\
\hline Uses speech & $96 / 110$ & $88 / 90$ & $184 / 200$ & 7.422 & $<0.01$ \\
\hline
\end{tabular}


Table 3. Comparison of chitdren (3-16 years) and adults (17-51 years) with PWS: behaviour.

\begin{tabular}{|c|c|c|c|c|c|}
\hline Item & $\begin{array}{l}3-16 \\
\text { years }\end{array}$ & $\begin{array}{l}17 \times 51 \\
\text { years }\end{array}$ & Total & $x^{2}$ & $p$ \\
\hline
\end{tabular}

\section{Feeding}

Eats normal amount of food

$\begin{array}{lllll}47 / 117 & 23 / 88 & 70 / 205 & 4.399 & <0.05 \\ 41 / 117 & 24 / 88 & 65 / 205 & 1.400 & \text { NS } \\ 29 / 117 & 41 / 88 & 70 / 205 & 10.619 & <0.01 \\ 29 / 114 & 28 / 89 & 57 / 203 & 0.898 & \text { NS } \\ 27 / 114 & 11 / 90 & 38 / 204 & 4.359 & <0.05\end{array}$

\section{Sleep}

Regular pattern of sleep

Repeated waking

Excessive daytime sleepiness

$\begin{array}{lllll}90 / 117 & 70 / 91 & 160 / 208 & 0.000 & \text { NS }\end{array}$

$\begin{array}{lllll}42 / 116 & 20 / 87 & 62 / 203 & 4.095 & <0.05\end{array}$

$19 / 117 \quad 38 / 88 \quad 57 / 205 \quad 18.162<0.001$

rightSocial behaviour

Isolated/in world of own

Too friendly with strangers

Unusual eye contact

Abnomal facial expression/gesture

Problems shopping

$\begin{array}{lllll}37 / 114 & 39 / 89 & 76 / 203 & 2.756 & \text { NS } \\ 75 / 112 & 45 / 90 & 120 / 202 & 5.955 & <0.02 \\ 35 / 113 & 28 / 88 & 63 / 201 & 0.016 & \text { NS } \\ 32 / 112 & 19 / 87 & 51 / 199 & 1.164 & \text { NS } \\ 40 / 114 & 37 / 91 & 77 / 205 & 0.670 & \text { NS }\end{array}$

Activity

Underactive

$\begin{array}{lllll}72 / 116 & 66 / 89 & 138 / 205 & 3.345 & \text { NS } \\ & & & \end{array}$

Normally active

$42 / 116 \quad 22 / 89 \quad 64 / 205 \quad 3.095 \quad$ NS

Overactive

Clumsy

Creates chaos aimlessly

$2 / 116 \quad 1 / 8$

$3 / 205$ NA

$68 / 111 \quad 48 / 89$

$116 / 200 \quad 1.089 \quad$ NS

$27 / 114 \quad 13 / 90$

$40 / 204$

2.724 NS

\section{Communication}

Echolalia

$\begin{array}{lllll}60 / 105 & 41 / 90 & 101 / 195 & 2.606 & \text { NS } \\ 33 / 110 & 30 / 91 & 63 / 201 & 0.204 & \text { NS } \\ & & & & \\ 40 / 115 & 24 / 90 & 64 / 205 & 1.549 & \text { NS } \\ 28 / 114 & 25 / 90 & 53 / 204 & 0.271 & \text { NS } \\ 73 / 115 & 74 / 91 & 147 / 206 & 7.911 & <0.01\end{array}$

Shouts or screams unexpectedly

$73 / 115-74 / 91$

$147 / 206 \quad 7.911<0.01$

\section{Aggression and self-injury}

Aggressive towards:

chilutren

$\begin{array}{lllll}15 / 114 & 7 / 91 & 22 / 205 & 1.578 & \text { NS } \\ 16 / 114 & 14 / 90 & 30 / 204 & 0.093 & \text { NS } \\ 67 / 114 & 65 / 91 & 132 / 205 & 3.535 & \text { NS }\end{array}$

Self-injurious behaviour 
An additional item asked about expressive speech; of the 87 adults wh PWS, 58 conversed with no problems, 19 used longsentences, 6 constructed sentences with more than 2 words and links, 3 used wo word sentences, and 1 person could only indicated objects with a single word.

\section{Discussion}

The sample of 226 children and adults with PWS is one of the largest reported, the other large questionnaire survey being that of 232 adults with PWS by Greenswag (1987) in the USA. The findings of these two studies are broadly consistent, and are also consistent with those reported in snaller studies employing matching to contrast subjects (Clarke et al. 1996). The overall pattern of emotional and behavioural problems differs from that found in Cri du chat syndrome by Cornish and Pigran (1996).

Excessive daytime sleepiness, self-injury and unusual routines seem more problematic for the older group in our sample, whereas stubbomness and verbal abuse seem to be less problematic. Problems with mood seem to be characterised by excessive happiness in the younger group and by low mood in the older group. Longitudinal studies will be necessary to confirm these findings, and to distinguish developmental from cohort effects. Other differences appear largely consistent with the results of skill acquisition during development (i.e. an improvement in self-care and related skills). Further studies are also necessary to investigate the effect of variables such as body mass index on behavioural problems. Early reports of behaviour in PWS often describe "stubbornness", but this did not emerge as a frequently reported problem in this sample. This may be because behaviours which may be perceived as contributing to stubbornness, such as repetitive questioning and insistence on routine, were identified more specifically in the SSBP-PQ. Both were quite prevalent in the sample reported here. In keeping with previous reports, underactivity was frequently reported in association with PWS. Only two males and one female were reported as overactive.

The study has a number of methodological weaknesses, including the lack of a control or contrast group (which would allow matching for age, gender and severity of intellectual disability), an exclusive reliance on carer-rated information, and a possible bias towards the over-inclusion of people with PWS from higher socio-economic graups (whose parents are probably more likely to join parent/carer organisations and to respond to requests for structured written information). The results reported are intentionally focused on problem behaviours, mather than positive behavioural or temperamental characteristics, because problem behaviours are clinically more important and often a focus of concern for parents, teachers and other carers. Inclusion of other characteristics may have 
reduced the response rate in view of the amount of information that would have had to be gathered. The authors are aware that many carers report positive temper-amental characteristics in association with PWS, and that there is considerable variation in the prevalence of problem behaviours between individuals and over ame,

Further research, investigating positive behavioural characteristics, would allow professionals to present a more accurate and rounded picture of the behavioural profile associated with PWS. Longitudinal studies would help to clarify changes in behaviour over time.

\section{Acknowledgements}

The authors are grateful to the PWSA (UK) for their invaluable support and practical help, to the carers who completed the SSBP-PQ, and to the people with PWS who agreed to take part in the study. We are also grateful to the University of Birmingham Department of Psychiatry and South Birmingham Mental Health Trust for access to data-analysis, photocopying and other facilities, and to Dr Greg O'Brien for the SSBP-PQ reliability data.

\section{References}

Boer H., Langton J. \& Clarke D. (1999) Development and behaviour in genetic syndromes: Rubinstein-Taybi Syndrome. Jounnal of Applied Research in Intellectual Disabilitices 12, 302-7.

Clarke D.J. (1995) Psychiatric and behavioural aspects of Prader-Willi syadrome in adult life. MD Thesis, University of Birmingham.

Clarke D J., Boer H., Chung M.C., Sturney P. \& Webb T. (1996) Maladaptive belaviour in Prader-Willi syndrome in adult life. Journal of Intdlectual Disability Research 40, 159-65.

Clarke D.J., Boer H., Webb T., Scott P., Frazer S., Vogels A., Borghgraef M. \& Curf. L.M. G.

(1998) Prader-Willi syndrome and psychotic symptoms: 1. Case descriptions and genetic studies. Joumal of Intellectual Disability Rescatch 42, 440-450.

Clarke D. J., Waters]. \& Corbett]. A. (1989) Adults with Prader-Willi syndrome: abnomalitices of sleep and behaviour. Journal of the Royal Society of Medicinc 82, 21-4.

Cornish K.M. \& Pigram J. (1996) Developmental and behavioural characteristics of cri du chat syndrome. Ardives of Disease in Childhood 75, 448-50.

Dittrich B., Robinson W. P., Knoblauch H., Buiting K., Schmidt K., Gillessen-Kaesbach G. \& Horsthemke B. (1992) Molecular diagnosis of the Prader-Willi and Angetrnan syndromes by detection of parent-of-origin specific DNA methylation in 15q11-13. Human Cenctics 90, 313-5. 
Dykens E.M., Hodapp R.M., Walsh K.K. \& Nash. L. (1992) Adaptive and maladaptive behavior in Pradet-Willi Syndrome. Joumal of the Amerian Academy of Child and Adolescent Psychiatry $31,1131-6$.

Dykens E.M. Leckman J.F. \& Cassidy S.P. (1996) Obsessions and compulsions in Prader-Willi syndrome. Joumal of Child Psychology and Psychatry 37, 995-1002.

Greenswag L.R. (1987) Adults with Prader-Willi syndrome: a survey of 232 cases. Divelopmental Medicine and Child Neurology $29,637-48$.

Harris J.C. \& Allen R.P. (1996) Is excessive daytime sleepiness characteristic of Prader-Willi syndrome? Archines of Paediatric and Adolescemt Medicine 150, 1288-93.

Hodapp R.M. (1997) Direct and indirect behavioral effects of different genetic disorders of mental retardation. American Joumal on Mental Retardation 102,67-79.

Holland A.J. Treastre J, Coskeran P. \& Dallow, J. (1995) Characteristics of the eating disorder in Prader-Willi syndrome: implications for treatment. foumal of Intellectual Disability Researh 39, 373-81.

Holland A.J., Treasure J., Coskeran P., Dallow J., Milton N. \& Hillhouse E. (1993) Measurement of excessive appetite and metabolic changes in Prader Willi syndrome. International Journat of Obesity 17, 526-32.

Kollrack H.W. \& Wolff, D. (1966) Paranoid-halluzinatorische Psychose bei Prader-Labhart-Willi-Fanconi-Syndrom. Acta Padopsyiatrica 33, 309-14.

O'Brien G. (1994) The developmentall and behavioural consequences of corpus callosal agenesis and Aicardi syndrome. In: Callosal Agenesis - The Natural Split Brain leds M. Lassonde, and M.A. Jeeves), pp. 235-246. Plenum Press, New York, NY.

O'Brien G. (1995) SSBP Postal Questionmaive. Society for the Study of Betuavioural Phenotypes. Northgate Hospital, Northumberland.

O'Brien G, and Yule W. (1995) Why behavioural phenotypes? In: Behaviowral Pherotypes (eds. G. O'Brien, and W. Yule), pp. 1-23. Mackeith Press, London.

Prader A. Labhart A., and Willi H. (1956), Ein Syndrom von Adipositas, Kleinwushs, Kryptorchismus und Oligophreme nach myatonieartigem Zustand im Neugeborenalter.

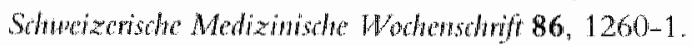


Chapter 5

\section{Prader-Willi syndrome, compulsive and ritualistic behaviours.}

The first population-based survey

Published as:

Clarke D.I., Boer H. Whittington J., Holland A., Buder J. \& Wobb T. (2002) Prader-Willi syndrome, compulsive and ritualistic behaviours: the first population-based survey. British Joumal of Psychiatry 180, 358-62.

*) Contributed equally to the study 
Backgrownd: Obsessive-compulsive disorder has been reported in assiociation with Prader-Willi syndrome.

Aims: To report the nature and prevalence of compulsive and similar symptoms associated with Prader-Willi syndrome in a population ascertained as completely as possible.

Mehod: Attempted complete ascertainment of people with Prader-Willi syndrome in 8 English counties. Administration of standardised rating scales and a structured interview. Comparison with people with learning disability and high body mass indices.

Results: Prader-Willi syndrome was associated with high rates of ritualistic behaviours such as the need to ask or to tell something, insistence on routines, hoarding and ordering objects, and repetitive actions and speech, compared to the control group; and was negatively correlated with intelligence quotient and socialization age. Typical obsessive-compulsive symptoms such as checking, counting and cleaning compulsions, or obsessional thoughts, were not found.

Condusions: $\mathbb{R}$ itualistic and compulsive behaviours occur more frequently in association with Prader-Willi syndrome than among people with intellectual disability and significant obesity.

Prader-Willi syndrome (PWS) is a disorder of genetic origin. About $70 \%$ of affected people have a deletion of $15 q 11-13,30 \%$ matemal uniparental disomy, and a very small proportion of people have an imprinting error affecting genes in the $\mathrm{q} 11 .-13$ area of chromosome 15 . It is characterised by a drive to over-eat, short stature and hypogonadism in adults, with failure to thrive, hypotonia and feeding difficulty in the neonatal period. Reports have described associations between PWS and emotional and behavioural disorders including abnomally frequent and severe outbursts of temper, mood abnormalities, psychotic disorders and obsessive-compulsive disorder (Whitman \& Accardo, 1987; Clarke ef al. 1996; Dykens et al. 1996; Clarke ef al 1998). We describe ritualistic, compulsive and similar symptoms documented during the first population-based study of PWS in the UK. Comparisons are made with a control group of people with similar severities of leaming disability and high body mass indices.

\section{Method}

The Canbridge University population study of PWS aimed to ascertain everyone with PWS who resided in the counties of Bedfordshire, Berkshire, Buckinghamshre, Cambridgeshire, Norfolk, Northamptonshire, Oxfordshire and Suffolk. Details of the method of ascertainment are given in detail elsewhere (Whitrington ef al. 1991). The study population consisted of about 5 million people (about one tenth of the population of England and Wales). Contact was 
made with the families and carers of people with PWS, and they were invited to participate in the study. The take-up rate was $64 \%$, and 65 people were identified. An additional 22 people with the syndrome were resident in the region but had moved there specifically to take up specialist services for people with PWS, or were recruited specifically to take part in the study; all these additional people with PWS have been included in the present study.

Standard checklists of obsessive and compulsive symptoms were not used in this study in view of their lack of reliability and validity when used to assess people with intellectual disability. Some people with PWS included in the study had severe intellectual disability, making the assessment of aspects such as distress caused by OCD symptoms (required by most standardised checklists) almost impossible. Questions were asked in a semi-structured interview, the PWS-Structured Interview Questionnaire (PWS-SIQ), lasting between 2.5 and 4 hours, conducted with the main carer of the person with PWS. Carers were encouraged to describe all behaviours they perceived as problematic. The questionnaire used included items relating to diagnosis, based on the consensus clinical criteria published by Holm ef al. (1991); behavioural problems; eating behaviour (including hoarding of objects as well as food); childhood psychiatric disorders (attention deficit, hyperactivity and autistic disorders); schooling; physical health and receipt of medication. Informant versions of the Developmental Behaviour Checklist (DBC) (Einfeldt, 1997), Aberrant Behaviour Checklist (ABC) (Aman et al. 1985a,b) and Vineland Adaptive Behaviour Scales (VABS), (Sparrow ef al. 1984) were administered. Anecdotes and examples were sought, and follow-up questions were used to clarify ambiguous statements. An appropriately trained investigator (JW) also spent time with the person with PWS and administered the appropriate Wechsler intelligence scales, and tests of attainment in reading, spelling and arithmetic. This gave an opportunity to observe the person with PWS and their reactions to the test situation, and provided additional information about attention and concentration. In view of the eating disorder associated with PWS, repetitive, preoccupying thoughts relating to food have been excluded from the results presented; almost everyone with PWS who was seen seemed to spend a great deal of tume thinking about food.

A comparison group consisting of people with learning disability and people who had volunteered to take part in the epidemiological study, but who were found not to have PWS on genetic or clinical grounds, has been used. Such a contrast group has the advantage of having similar overall severities of cognitive impairments, and high body mass indices (see Table 1). The use of such a comparison group allows an estimate of the prevalence of obsessive and compulsive symptoms associated with PWS (rather than with obesity or intellectual disability). The same measures and assessments were used for the comparison group. 
Table 1. Characteristics of PWS and comparison groups

\begin{tabular}{lll}
\hline & PWS & ID contrast \\
\hline Age band & & \\
$0-15$ years & 33 & 22 \\
$16-30$ years & 40 & 9 \\
31 years and over & 24 & 12 \\
Mean age (S.D.) & $20.8(12.5)$ & $20.2(14.6)$ \\
& & \\
10 band & 15 & 9 \\
$0-50$ & 20 & 9 \\
$51-60$ & 26 & 8 \\
$61-70$ & 17 & 5 \\
$71-80$ & 6 & 9 \\
$80+$ & $63(12.3)$ & $64(17.7)$ \\
Mean rO (S.D.) &
\end{tabular}

Note. Not all participants agreed to provide al information (e.g. to undertake Ia testing)

The hypothesis that compulsive symptoms are associated with developmental delay, as suggested by Dykens et al. (1996) would be consistent with an age-related decline in such symptoms, or with a ceiling effect, such that development and compulsive symptoms "stick" at a stage of development unaffected children pass through. This hypothesis was tested in the present study by correlating compulsive behaviours with chronological age, with IQ (as a measure of mental age) and with Vineland Adaptive Behaviour Scale score (a measure of socialization age).

Two measures of compulsive symptoms were formed: a simple count of the number of symptoms from the list in Table 1 , plus "needs routine" and "anticipation" (endorsed by informants) and a weighted count in which those behaviours rated as a severe problem were given a count of 2 , whereas those rated as a problem were given a count of 1 . The sample was divided into the age bands 5 to 12 years, 13 to 19 years, and 20 years and over. IQ was defined as the full-scale score on the age-appropriate Wechsler ability test. Socialization age was defined as the age - equivalent score on the Vineland Adaptive Behaviour scales. Obesity was assessed using the Body Mass Index (BMI: weight in $\mathrm{kg}$ divided by height in metres squared). For adults, the maximum BMI was also recorded, where known. Mood swings were assessed using the sums of scores of carer-rated items relating to "mood sings - ever" on the PWS-SIQ (rated 0-4) and the score on the Aberrant Behavior Checklist item "mood changes rapidly" (for adults) or the Developmental Behaviour Checklist item "mood changes rapidly for no apparent reason" (for children). Anxiety and depression were assessed by the sums of the scores for items "ever had severe anxiety lasting more than a few days", "ever had severe depression lasting more than a few days", "ever had other nervous problem lasting more than a few days" (all items from the PWS-SIQ) "exhibits excessive unhappiness" (Vineland Maladaptive Behaviour Scale) and "depressed mood" (Aberrant 
Behavior Checklist for aduilts, Developmental Behaviour Checklist for children). Autistic traits were assessed using the sum of the scores from 6 PWS-SIQ items with the stem "hardly ever": "initiates conversation", "calls attention to things", "smiles in response", "co-operates in play", "makes eye contact", "shows imaginative play", and the items "has repetitive talk" and "has little emotional expression".

\section{Results}

The male to female ratio for the PWS group was $1.29: 1$ and for the intellectual disability contrast group 1:1. The mean body mass index for the PWS group was $31.6 \mathrm{kgm}^{-2}$ (S.D. 11.8) and for the intellectral disability comparison group 28.3 $\mathrm{kgm}-2$ (S.D. 10.1). Information regarding the ages and intelligence quotients of the PWS and contrast groups is summarised in Table 1. The prevalence of compulsive symptoms in the PWS and contrast groups is compared in Table 2.

Compulsive symptoms did not decline with age in the PWS sample, and were not correlated with obesity (BMI or maximum BMI), with (long term) anxiety/depression or with severity of eating behaviour. The latter finding may mask a ceiling effect because all people with $P W S$ have some problems with appetite regulation. There were significant positive correlations with (short term) mood swings, $(r=0.23, p=0.05$ for weighted compulsion count) and autistic symptoms $(r=52, p=0.000$ for weighted compulsion count). There were significant negative correlations with IQ $(r=-0.30, p=0.008$ for weighted count) and socialization age $(\mathrm{r}=-0.30, \mathrm{p}=0.002$ for weighted count $)$. An examination of correlations between weighted compulsion counts and IQ, socialization age and

Table 2. Obsessive-compulsive symptoms rated very frequent or very severe.

\begin{tabular}{|c|c|c|c|c|c|c|c|c|c|}
\hline \multirow[t]{2}{*}{ Symptom } & \multicolumn{2}{|c|}{ PWS1 $(n=93)$} & \multicolumn{2}{|c|}{ PWS pop2(n-68) } & \multicolumn{2}{|c|}{ Contrast (n=42) } & \multirow[t]{2}{*}{$\times 2$} & \multirow[t]{2}{*}{ d.f } & \multirow[t]{2}{*}{ p } \\
\hline & $n^{3}$ & $(\%)$ & $n_{3}$ & $(\%)$ & $n 3$ & $(\%)$ & & & \\
\hline Need to ask or tell & $36 / 78$ & $(46.2)$ & $27 / 55$ & $(49.1)$ & $4 / 29$ & $(13.8)$ & 9.4 & 1 & $<0.01$ \\
\hline Routines & $26 / 80$ & $(32.5)$ & $17 / 57$ & $(29.8)$ & $4 / 33$ & $(12.1)$ & 5.0 & 1 & $<0.05$ \\
\hline Hoarding & $19 / 80$ & $(23.7)$ & $12 / 57$ & $(21.1)$ & $1 / 33$ & $(3.0)$ & 6.9 & 1 & $<0.01$ \\
\hline Repetitive & $18 / 80$ & $(22.5)$ & $14 / 57$ & $(24.6)$ & $3 / 33$ & $(9.1)$ & 2.8 & 1 & NS \\
\hline Ordering & $11 / 80$ & $(13.7)$ & $11 / 57$ & $(19,3)$ & 0 & & 5.0 & 1 & $<0.05$ \\
\hline Cleaning & $2 / 80$ & $(2.3)$ & $1 / 57$ & $(1.8)$ & 0 & & 0.9 & 1 & NS \\
\hline Counting & 0 & & 0 & & 0 & & - & & $\mathrm{NS}$ \\
\hline Checking & 0 & & 0 & & 0 & & - & & NS \\
\hline
\end{tabular}

1. PWS refers to total number of people with PWS assessed.

2. PWS pop refers to people with PWS ascertained in the total population survey only.

3. The value of $m$ varies because not all Items are appropriate to all people taking part (e.g. need to ask is not applicable to people without speech): 
autistic symptoms individually, controlling for the effect of the other two variables, showed no significant correlations other than for autistic symptoms (controlling for IQ and socialization) $(r=0.34, p=0.005)$. No significant correlations were found for the contrast group. Some stereotyped and ritualistic bethaviours were seen duning direct observation of the people with PWS who took part in the study. No tics were noted.

\section{DISCUSSION}

Compulsions and Prader-Willi syndrome

The results of this first epidemiological survey of compulsive symptoms associated with PWS are broadly in agreement with an earlier study from the United States of America (Dykens et al 1996). It seems likely that the compulsive behaviours associated with PWS are similar to the compulsions seen in early childhood in children without developmental disabilities. As in the earlier studies, very few obsessional thoughts were reported, and the range of compulsive symptoms described was relatively restricted, with few symptoms such as counting, cleaning or checking. The paucity of obsessional symptoms noted in our study may reflect a difficulty for people with PWS and their carers in describing such symptoms, but the pattern of symptoms observed is also similar to that seen in early childhood.

Compulsive symptoms were found to be much more prevallent in our study groups of people with PWS than in the contrast group of people with similar severities of intellectual disability, who were of similar ages and who had high body mass indices. It is, therefore, unlikely that the high rate of compulsive symptoms is accounted for by the relative obesity of many people with PWS, or by the presence ofintellectual disability. Taken with other information about the clinical features and behavioural characteristics of populations of people with PWS, there is some evidence for a constellition of features (labile mood, vulnerability to loss of temper, ritualistic and compulsive symptoms, repetitive questioning and insistence on routine) similar to the attributes of children without developmental disability in early childhood.

Dykens ef 4. (1996) described 91 people with PWS, aged 5 to 47 years (mean 19 years), with IQs ranging from 50 to 89 (mean 69) who were recruited at PWS Association meetings and through support groups. The findings were compared to those for 43 people who did not have intellectual disability but did have a clinical diagnosis of OCD and who were recruited from three clinics for people with OCD. The PWS group were rated using a modified (infomant) version of the Yale-Brown Obsessive Compulsive Scale (Y-BOCS) (Goodman et al. 1989a,b), completed by their main carer, and the OCD group completed the standard 
(self-report) version of the $\mathrm{Y}-\mathrm{BOCS}$. In both versions of the instrument, $56 \mathrm{symp-}$ toms were rated as being present in the last week, or ever (analyses being based on the ratings for the last week). Ten additional items in the $\mathrm{Y}-\mathrm{BOCS}$ rated symptom severity, including the extent to which symptoms were time consuming, distressful, out of control, or causing social or occupational impairment. Informants reported high rates in the PWS group of compulsions concerning hoarding $(58 \%)$, a need to tell or ask (53\%) and ordering, arranging and repeating rituals (37-38\%). Other compulsions reported included cleaning (24\%), counting (17\%) and checking (15\%). Obsessions were also reported, but were less prevalent. Informants reported compulsive behaviours causing "moderate" or "severe" distress in $64 \%$ of people, adaptive impairments in $80 \%$ and excessive time-consumption in $45 \%$, using the $\mathrm{Y}$-BOCS scaling. Comparisons with the OCD clinic sample indicated significant differences $(p<0.05)$ for two compulsions that were commoner in the PWS group; hoarding (79\% vs $7 \%$ ) and needing to tell or ask ( $51 \%$ vs $23 \%$ ). Checking behaviour was less common in the PWS group (16\% vs $55 \%$ ); all other ratings did not differ significantly between the two groups. The authors concluded that "increased risks of OCD are strongly indicated in people with PWS, based on the range and severity of symptoms encountered in this sample." They also noted that some diagnostic criteria for OCD, including DSM-IV (American Psychiatric Association, 1994), do not include the criterion of the person's recognition that their symptoms are excessive or unreasonable in the case of children, and argued that people with PWS may also have less insight into their OCD symptomatology because of their cognitive limitations. Dykens ef al. (1996) also noted that the pattern of symptoms they found to be associated with PWS loaded on only one factor (the principle factor) that emerged from a factor analysis of $\mathrm{Y}-\mathrm{BOCS}$ ratings of 107 patients with obsessive-compulsive disorder (OCD) (Baer, 1993). This factor includes aspects such as hoarding, repeating rituals, and concerns with symmetry, exactness, ordering and arranging. Feurer of al. (1998) reported that analysis of the Compulsive Behavior Checklist (CBC) scores of people with PWS yielded only one general factor, with the exception of an item relating to "deviant skin-grooming-skin picking."

\section{Compulsive symptoms and child development}

The prevalence of obsessional and compulsive symptoms varies throughout childhood. Bedtime and dressing rituals are common in early childhood (Gesell et al. 1974). Other rituals and compulsive-like phenomena may occur later in child-

hood. The prevalence of obsessional disorders, as distinct from compulsive acts, has been estimated at between $0.2 \%$ and $12 \%$ of clinical populations of children and adolescents (Judd, 1965; Hollingsworth et al. 1980). Zohar \& Bruno (1997) studied 1083 school children aged 8 to 13 years in Jerusalem using the Maudsley 
Obsessive-Compulsive Inventory. They found that obsessional ideas and compulsive behaviours were common among children at the age of 8 , but were present in only a minority of children aged 13 years. Evans et al. (1997) used the Child Routines Inventory to assess compulsive-like behaviour in children, and found that children between 2 and 4 years of age had higher rates of such behaviours than children aged below 1 year or above 4 years.

Goodman ef al. (1989a) reported the presenting symptoms among 70 consecutive children and adolescents with a primary diagnosis of $\mathrm{OCD}$, and found the most common obsessions to be those conceming contamination by dirt or germs $(40 \%)$, worries about something terrible happening (24\%) and worries about symmetry, order and exactness (17\%). The most commonly reported compulsions were those concerning excessive or ritualised hand washing, showering, bathing, tooth brushing or grooming (85\%), repeating rituals (e.g. going in and out of a doorway) (51\%) and checking compulsions (46\%). Compulsions regarding ordering or arranging were found in $17 \%$, counting in $18 \%$ and hoarding or collecting in $6 \%$. These studies suggest a shift during childhood from relatively common rituals and compulsive-me behaviours in early childhood to low rates of obsessional and compulsive symptoms resembling those of adult $O C D$ among vulnerable children later in childhood and in adolescence.

\section{Serotonin and compulsive and ritualistic behaviours}

Obsessive-compulsive symptoms or OCD may occur in the context of depressive illness, and its response to antidepressants such as clomipramine and specific serotomin reuptake inhibitors (SSRIs) as well as evidence from neurochemical studies, suggests the involvement of the serotonergic system in the genesis or maintenance of OCD (Goodman et al. 1991; Riddle et al. 1992; Zohar et al 1987; 1988). Although abnormalities in serotonergic systems seem to play a part in the genesis of some OCD, it seems likely that the anti-obsessional effect of drugs acting on serotonergic systems may result from alterations in the balance between serotonin and other neurotransmitters, or changes in receptor functioning (Murphy et al. 1989). One study has reported abnomal serotonin tumover associated with PWS, with increased concentrations of serotonin metabolites in the cerebrospinal fluid of children and adolescents with PWS compared to comparison groups (Akefeldt ef al. 1998).

\section{Dxytocin}

Leckman et al. (1994) reported elevated cerebrospinal tuid oxytocin concentrations in association with obsessive-compulsive disorder in people without intellectual disability. A reduction in the number of oxytocin-containing neurons in 
the paraventricular nucleus of the hypothalamus has been found in post mortem studies of some people with PWS (Swaab et al. 1995).

\section{Genetics}

Prader-Willi syndrome is thought to result from genomic imprinting, with the absence of the paternal contribution to genes in the q11q 13 area of chromosome 15. The finding of high rates of ritualistic behaviour, together with other reports of psychiatric disorder associated with the syndrome, may be of relevance to understanding of the genetic and metabolic basis of such disorders in the general population.

\section{Clinical implications}

Prader-Willi syndrome is associated with a relatively high prevalence of ritualistic behaviours, but not with typical obsessive-compulsive disorder The pattern of symptoms seen is similar to that seen in young children without PWS

Prader-Willi syndrome is caused by the non-expression of the paternal contribution of genes in the q11q13 area of chromosome 15; the finding of high rates of ritualistic belhaviour may be of relevance to understanding of the genetic basis of compulsive and allied disorders

\section{Limitations}

A proportion of people included in the study had limited language ability, making the assessment of obsessional thoughts difficult.

The study was carried out in England, and cultural influences on ritualistic and compulsive behaviours may affect the prevalence of such disorders associated with Prader-Willi syndrome in other countries.

Prolonged observation to confirm carer reports was not possible

\section{References}

Åkefeldt A., Ekman R., Gillberg C. \& Mânsson J.E. (1998) Cerebrospinal fluid monoawiutes in Prader-Will syndrome. Biological Psychiatry 44, 1321, 8 .

Singh N.N., Stewart A.W. et al. (1985a) T7te Aberrant Behawor Checklist: A behavior rating sanle for the assesshest of treatment effects. American Joumal of Mental Deficiency 89, 485-91.

Aman M.G., Singh N.N., Stewart A.W. et al. (1985b) Psychomeric dhatacterishics of dhe Albcrant Behavior Cheoklist. American Joumal of Mental Deficiency 89,492-502. 
American Psychiatric Association (1994) Diagnostic and Statistical Manual of Mental Disonders, Fourth Edition. APA, Washington, DC.

Bater L. (1993) Fador andysis of sympton subtypes of obsessiwe-conpulsiwe disorder and their relation to personality and tic disorders. Journal of Clinical Psychatry, 55, 18-23.

Clarke D.J., Boer, H., Chung, M.C. et al. (1996) Maladaptiwe behaviou in Prader-Will syndrome in aduli life. Joumal of Intellectual Disability Research, 40, 159-65.

Clarke D.J., Boer H., Webb T. et al. (1998) Prader-Willisyndrome and psychotit symptonts 1. Case descriptions and genetic studies. Joumal of Intellectual Disability Research 42, 440-50.

Dykens E.M., Leckman J.F. \& Cassidy S.B. (1996) Obsessions and conpulsions in Prader-Will symdrome. Joumal of Child Psychology and Pychiatry, 37, 995-1002,

Einfeldt S.L. \& Tonge B.J. (1993) Mamal for the Developwental Behavour Checlist. Centrefor Developwental P5ythatry, Monash University, Mellbourne and School of Psychiatry, University of Now South Wales, Sydney.

Evans D.W., Leckman J.F., Carter A, et al. (1997) Ritual, habit and peffectionism: The prevalence and dovelopmon of rompulsine-like behavior in nomal young wildren. Child Development 68, $56-68$.

Feurer I.D., Dimitropoulos A., Stone W. L. et al (1998) The latent mandestucture of the Compusive Behowiour Checklist in people with Prader-Willi syndropme. Journat of Intellectuall Disability Research, 42, 472-80.

Gesell A. Ames L.B. \& Ilg F.L. (1974) Infant and the Child in Culture Today. Harper \& Row, New York.

Goodman W.K., Price L.H., Rasmussen S.A. et al. (1989a) The Yale-Bronn Obsessive Compul

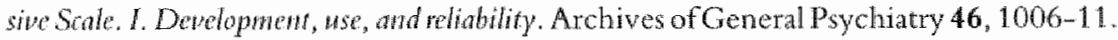

Goodnan W. K. Price L.H., Rasmussen S.A. et al. (1989b) The Yale-Broun Obsessine Compulsive Sale. 11. Validity. Archives of General Psychiatry 46, 1012-6.

Goodnan W.K., Price L.H., Woods S.W. et al. (1991) Pharnacologic challenges in obsessinewompulsive disorder. In: The Psychobiology of Obsessive-Compulsive Disorder (eds J. Zohar, T. Insel \& S. Ratunussen/, pp. 162-86. Springer, New York, NY.

Hollingsworth C.E., Tanguay P.E., Grossman L. et all (1980) Longwem ontome of obsessite-compulsive disorders in childhood. Joumal of the American Academy of Child and Adolescent Psychiatry $19,134-44$.

Holm V.A., Cassidy S.B., Butler M.G. etal, (1993). Prader-Whi Symdrome. Consensas Diagnosic Cufiena. Pediatrics $91,398-402$.

Judd L. (1965) Obsessine-compulsive newosis in dildren. Archives of General Psychiatry 12. $136-43$

Leckman J F., Goodman W.K., North W.G. et al. (1994) Elowgted cerebrospinal fluid level of oxptocis in abesine-compulsive disowder. Archives of General Psychiatry, 51, 782-92.

Murphy D., Zohar J., Pato M. et al. (1989), Obsessive-compulsive disorder as a $5 \mathrm{HT}$ subsysem behawowal disorter. British Joumal of Psychiatry 155 suppl. 8], 15-24.

Riddle M.A., Scahill L., King R.A. et al. (1992) Double-blind, orossover trial of fluoxetine and phecto in dildren and adolescents with obsessive-compulsibe disorder. Journal of the American Academy of Child and Adolescent Psychiatry 31, 1062-9. 
Spanow S.S., Balla D.A., Cicchetri D.V. (1984) Vinchand Adaptive Buhwour Scales: A Revision of the Vineland Social Maturity Scale by Edgar A. Doll. American Guidance Service, Circle-Pines, Minnesota.

Swaab D.F., Purba J.S. \& Hofman M.A. (1995). Alterations in the hypoihalawic parawentrialar

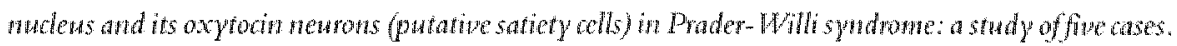
Journal of Clinical Endocrinology and Metabolism 80, 573-79.

Whitman B.Y and Accardo P. (1987) Emotional symptoms in Prader-Will syidrome adoleccents. American Joumal of Medical Genetics 28, 897-905.

Whittington J.E., Holland A.J., Webb T., Butler J.V., Clarke D.J., Boer H. (2001) Population prevalence and estimated birth incidence and mortality rate for people with Prader-Willi Syatrome in whe UK Healh Region Joumal of Medical Genetics 38, 792-8.

Zohar A.H. \& Bruno R. (1997) Normative and pathological obsessive-compulstive behavior and ideation in childhood: a question of timing. Journal of Child Psychology and Psychatry 38 , $993-9$

Zohar J., Insel T., Zohar-Kadouch R, et al. (1988) Serofonergic responsivity in obsessive-compulsive Wisorder: affect of chronic donviphamine treatmen. Archives of General Psychiatry 45, 167-72.

Zohar J., Mueller E.A., Insel T.R., et al. (1987) Serotonergic responsivity in obsessive-compulsive disorder: comparison of paticots and heathy onatrols. Archives of General Psychiatry 44, 946-51. 

Chapter 6a

\section{Prader-Willi syndrome and psychotic symptoms:}

Case descriptions and genetic studies

Published as:

Clarke , D.J., Boer*, H., Webb T., Scott P., Frazer S., Vogels A., Borghgraer M. \& Curfs L.M.G. (1998) Prader-Will syndrome and psychotic symptoms: 1. Case descriptions and genetic studies. Joumal of Intellectual Disability Research 42, 440-58.

* Contributed equally to the study 


\section{Abstract}

Six people with Prader-Willi syndrome (PWS) who developed psychoses are described. Along with other hiterature reviewed in the present paper, the resuls imply an association between PWS and psychotic symptoms. Genetic studies were possible in five cases and SNR.PN expression was examined in three cases. Matemal uniparental disomy and $15 q 11913$ deletions were found, demonstrating that psychotic symptoms are not associated with a single type of genetic abnomality.

The people in this report are identificd as ' $F$ to $K$ ' to distinguish them from the cases described by the authors in previous reports (Clarke 1993; Clarke et al. 1995).

\section{Introduction}

Prader-Willi syndrome (PWS) (Prader et al. 1956) is a disorder with at least two distinct clinical phases. Immediately after birth, affected infants are very hypotonic ("loppy") and have feeding difficulties. In early childhood, these children develop an almost insatiable appenite, which may lead to the consumption of such items as frozen or pet food, or to the stealing of food. Adults with PWS are of short stature with hypogonadism (failure of sexual development) and a characteristic appearance. The latter typically includes small hands and feet, a tent-like mouth and almond-shaped eyes. Obesity is often, but not invariably, a consequence of the eating disorder. In addition to the physical characteristics, a number of behavioural and emotional characteristics have been reported in association with PWS, including sleep abnomalities, temper tantrums or rages, skin-picking, and labile mood (Greenswag 1987; Clarke et al. 1989). Temper tantrums, self-injury, impulsiveness, lability of mood, inactrity and repetitive speech are significantly more problematic among adults with PWS than among contrasting subjects matched for age, gender and global severity of intellectual disability (Clarke et al. 1996). The syndrome is known to be associated with deletions of paternal origin affecting chromosone 15 at $15 q 11913$ and with matemal uniparental disomy (the inheritance of two chromosome 15 s from the mother instead of one from each parent). The 15 q 11 q 13 region, which encompasses the areas implicated in both PWS and Angelman syndrome, demonstrates imprinting. The PWS region, which lies nearer to the centromere, is matemally imprinted but paternally active. The opposite is true of the more distally located Angelman syndrome region. Imprinting explans why PWS can be the result of either external deletion or maternal uniparental disony (Robinson of al 1991). More rarely, PWS can result. from an error in resetting the imprinting pattern during meiosis so that the paternally inherited copy of $15 \mathrm{q} 1 \mathrm{lq} 13$ carres a matemal imprint, and consequently, 
fails to be expressed. Three paternally expressed genes, the small ribonucleoprotein $N$ gene (SNRPN), IPW and ZNF127, and two paternally expressed DNA fragments, PAR-1 and PAR-5, have been shown to lie within the PWS chromosomal region (Sutcliffe et al. 1994; Wevrick el al. 1994). The relative position of loci within the PWS and Angelman syndrome critical regions is illustrated in Figure 1.

\section{Previous reports of PWS associated with psychotic symptoms}

The first report of psychosis associated with PWS is believed to be that published in German by Kollrack \& Wolff (1966). These authors described a man who was 20 years old when he was admitted to hospital with a psychosis. Kolliack \& Wolff (1966) provided a detailed account of his symptoms and signs, and although genetic testing was not possible at the time, there seems little doubt that their patient had PWS. Six weeks before admission, he became agitated and 'with a bright red face he kept talking incessantly, uttering threats such as "ifl get them I'll hit them, I'll show them"." This subject had previously had a placid temperament, but whilst psychotic, he tried to attack his neighbours. He believed that the police were pursuing him, that he had been accused of stealing rabbits, that an old woman with a scrubbing brush was planning to kill him, that a child's grave in the local cemetery had been moved with some evil purpose in mind and that the tram system wiring in his home city had been deliberately made faulty to cause an accident. The subject apparently heard people talking who were not actually present. He was treated with a benzodiazepine and an antipsychotic, and improved in about 10 days. Kollrack \& Wolff (1966) commented that classification of the psychosis was difficult and concluded that their patient had an "endogenous agitated-depressive paranoidly coloured disorder'. It seems likely that the patient had an affective or cycloid psychosis.

Clarke (1993) reported three cases of psychosis associated with PWS. The first person was a 17 -year-old man with del $15 \mathrm{q} 11 \mathrm{q} 13$. His psychosis hach started suddenly 2 weeks before he was interviewed. The subject said that he was being 'got at' by 'Kevin', and paced round the house in an agitated manner saying that Kevin was shouting bad language in his ear and that his father's car had been stolen. He repeatedly asked if 'the dogs have been fed'. The family had no dogs. When interviewed, he said that 'Kevin' was kicking his leg (without being present) and making him lose his voice by shouting bad language in his car. His family doctor had prescribed chlorpromazine, but the subject had not taken any because his family were concerned that he might become dependent on it. He was encouraged to take the prescribed medication and the psychosis resolved 24 days after the onset with no recurrence to date. 


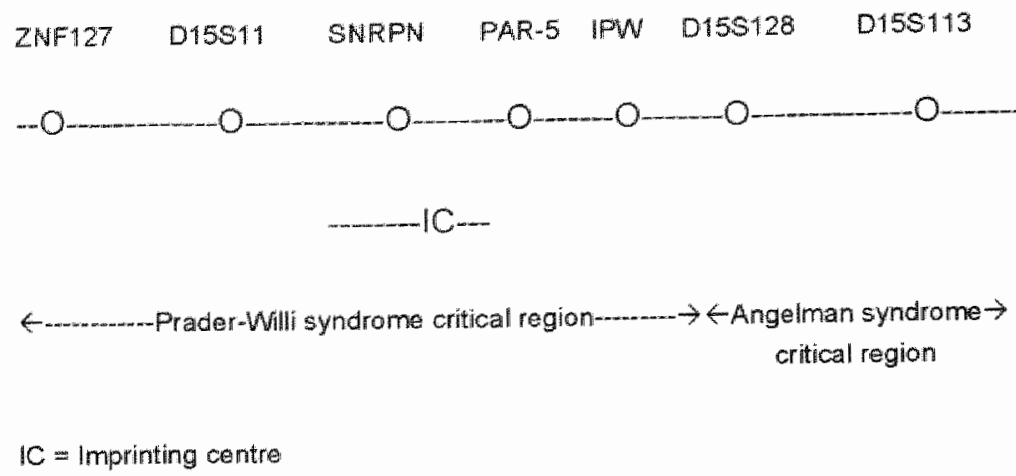

Figure 1 Relative positions of loci within the Prader-Will/Angelman syndrome critical region of chromosame 15. IC: Imprinting Centre.

Table 1. Physical characteristics

\begin{tabular}{|c|c|c|c|c|c|c|}
\hline Characteristic & $\begin{array}{l}\text { Case I } \\
(\mathbb{F})\end{array}$ & $\begin{array}{l}\text { Case } 2 \\
(G)\end{array}$ & $\begin{array}{l}\text { Case } 3 \\
(\mathrm{H})\end{array}$ & $\begin{array}{l}\text { Case } 4 \\
\text { (I) }\end{array}$ & $\begin{array}{l}\text { Case } 5 \\
\text { (J) }\end{array}$ & $\begin{array}{l}\text { Case } 6 \\
(K)\end{array}$ \\
\hline Gender $(M / F)$ & $F$ & $\mathrm{~F}$ & $\mathrm{~F}$ & $M$ & $M$ & $\mathbb{F}$ \\
\hline Vear of birth & 1968 & 1952 & 1970 & 1978 & 1971 & 1968 \\
\hline Height $(m)$ & 1.57 & 1.47 & 1.38 & 155 & 158 & 1.6 \\
\hline Weight at onset of psychosis $(\mathrm{kg})$ & 102 & 63.5 & 57 & 67 & 85 & $?$ \\
\hline Maximum weight $(\mathrm{kg})$ & 108 & 76.7 & 73 & 73 & 85 & 72 \\
\hline Neonatai hypotonia & ++ & ++ & ++ & ++ & ++ & + \\
\hline Tube feeding as neonate & - & ++ & - & +4 & + & + \\
\hline Hypogonadism & $\#+$ & ++ & ++ & + & +4 & + \\
\hline $\begin{array}{l}\text { Facial appearance typical of } \\
\text { Prader-Will syndrome }\end{array}$ & ++ & + & +4 & +4 & + & ++ \\
\hline Palm length $(\mathrm{cm})$ & 7.6 & 8.1 & 8.6 & 10.2 & 10.5 & 95 \\
\hline Mid-finger lengtith $(\mathrm{cm})$ & 6.4 & 6.7 & 5.6 & 7.6 & 6.5 & 6.1 \\
\hline obesity in childhood & 4 & + & ++ & (diet) & +4 & ++ \\
\hline Obesity in adult life & +4 & + & ++ & ++ & ++ & + \\
\hline
\end{tabular}

* Key: (?) information not availiable: (++) present and prominent; (+) present, not prominent; and $(-)$ not present.

The second case was a 24-year-old woman with PWS (del 15q11q13) who returned home one day from an industrial therapy unit, pointed to a fragment of scrap metal in her clothing, and repeatedly said, "I have not stolen it.' She became anxious, agitated and tearful. 'The subject refused to eat, and spent the night repeating phrases such as 'I'm being burned'. She was admitted to a psychiatric hospital. No organic cause was found for her symptoms. The subject was prescribed chlorpromazine and made a good recovery. She has remained free of psychotic symptoms, but has received a depot antipsychotic for many years to help her control outbursts of temper, which were worse perimenstrually. 
Table 2. Genetic and behawioural characteristics

\begin{tabular}{|c|c|c|c|c|c|c|}
\hline CHaracteristic & Case $1(b)$ & Case 2. (6) & $\operatorname{Case} 3(H)$ & $\operatorname{case} 4(1)$ & Case 50 & Case $6(k)$ \\
\hline Genetic abnormality del & $15 q 11013$ & $\begin{array}{l}\text { No expression } \\
\text { of SWRPN or } \\
\text { PAR-S (see } \\
\text { text) }\end{array}$ & $\begin{array}{l}46 \times(10.15) \\
\text { No expression } \\
\text { of SNRPDN or } \\
\text { PAR-5 }\end{array}$ & $\begin{array}{l}\text { Maternal } \\
\text { uniparental } \\
\text { disomy }\end{array}$ & gel $15 \mathrm{q} h \mathrm{lgl}$ & $?$ \\
\hline Sleep disorder (s) & $\begin{array}{l}\text { Daytime } \\
\text { sleepiness }\end{array}$ & $\begin{array}{l}\text { Sleeps after } \\
\text { Lunch }\end{array}$ & Early waking & None & $\begin{array}{l}\text { Daytime } \\
\text { steepiness }\end{array}$ & $\begin{array}{l}\text { Daytime } \\
\text { sletepirass }\end{array}$ \\
\hline Hyperphagia in adutt lise & + & ++ & +4 & + & +4 & $4+$ \\
\hline $\begin{array}{l}\text { obsessional ar compulsive } \\
\text { symproms/behaviours }\end{array}$ & + & - & . & + & + & $-\infty$ \\
\hline $\begin{array}{l}\text { thaAbnormally severe } \\
\text { tempers. }\end{array}$ & $H$ & - & $\cdot+4$ & 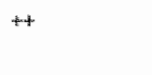 & +4 & $+a$ \\
\hline Sikn picking in adult life & +4 & $\infty$ & - & 。 & $+n$ & - \\
\hline Under-or overactivity & Underactivity & Underactivity & Underactivity & Underactivy & Underactivity & Underactivity \\
\hline
\end{tabular}

*For key, see Table 1

Table 3. Cognitive and adaptive skills

\begin{tabular}{|c|c|c|c|c|c|c|}
\hline skinll & $\begin{array}{l}\text { Caste I } \\
\text { (F) }\end{array}$ & $\begin{array}{l}\text { Case ? } \\
\text { (G) }\end{array}$ & $\begin{array}{l}\text { Case } 3 \\
(1))\end{array}$ & $\begin{array}{l}\text { Case } 4 \\
\text { (I) }\end{array}$ & $\begin{array}{l}\text { Case } 5 \\
\text { (I) }\end{array}$ & $\begin{array}{l}\text { Case } 6 \\
\text { (K) }\end{array}$ \\
\hline $\begin{array}{l}\text { Severity of intellectual } \\
\text { disability (ICD-10 clinical } \\
\text { guitelines) }\end{array}$ & Mild & Modemate & Moderate & Mild & $\begin{array}{l}\text { Moderate (10 } \\
40)\end{array}$ & $\begin{array}{l}\text { Mild/Moderate } \\
\text { (IO } 53 \text { ) }\end{array}$ \\
\hline Excessive speech & $\begin{array}{l}\text { Complete } \\
\text { sentences }\end{array}$ & $\begin{array}{l}\text { Complete } \\
\text { sentences }\end{array}$ & $\begin{array}{l}\text { Camplete } \\
\text { sentences }\end{array}$ & $\begin{array}{l}\text { Complete } \\
\text { sententces }\end{array}$ & $\begin{array}{l}\text { Complete } \\
\text { sentences }\end{array}$ & $\begin{array}{l}\text { Complate } \\
\text { sentences }\end{array}$ \\
\hline Self-care skillis & No deficits & No deficits & Adequate & $\begin{array}{l}\text { Declined since } \\
\text { psychosis }\end{array}$ & $\begin{array}{l}\text { With } \\
\text { supervision }\end{array}$ & $\begin{array}{l}\text { With } \\
\text { stipervision }\end{array}$ \\
\hline \multicolumn{7}{|l|}{ Wineland Adaptive } \\
\hline \multicolumn{7}{|l|}{ Behaviour 5cale Standard } \\
\hline \multicolumn{7}{|l|}{ Scores: } \\
\hline Communication & 65 & 20 & $<20$ & 42 & $?$ & $?$ \\
\hline Dally living & 66 & 38 & 24 & 61 & $?$ & $?$ \\
\hline Sociatization & 53 & 68 & 29 & 79 & $?$ & $?$ \\
\hline Total & 56 & 39 & 21 & 56 & $?$ & $z$ \\
\hline
\end{tabular}

* For key, see Table 1

Table 4. Problem behaviours and psychiatric diagnoses

\begin{tabular}{|c|c|c|c|c|c|c|}
\hline Behaviaur & $\begin{array}{l}\text { Case } \mathbb{1} \\
\text { (F) }\end{array}$ & $\begin{array}{l}\text { Case } 2 \\
\text { (G) }\end{array}$ & $\begin{array}{l}\text { Case } 3 \\
(\mathrm{H})\end{array}$ & $\begin{array}{l}\text { Case } 4 \\
\text { (1) }\end{array}$ & $\begin{array}{l}\text { Case } 5 \\
\text { (I) }\end{array}$ & $\begin{array}{l}\text { Case } 6 \\
(K)\end{array}$ \\
\hline
\end{tabular}

Aberant Behawiour Checklist

sub-scalle scores:

\begin{tabular}{|c|c|c|c|c|c|c|}
\hline (1) Irratability/agitation & 23 & 3 & 12 & $\gamma$ & 13 & 10 \\
\hline (11) Lethargy/whthdrawal & 6 & 9 & 8 & 9 & 21 & 15 \\
\hline (III) Stereotypical behawiours & 4 & 0 & z & 7 & 5 & $z$ \\
\hline (W) Hyperactivity/non-compliance & 11 & 3 & 7 & 4 & 30 & 13 \\
\hline (V) Inappropriate speech & 3 & $4_{i}$ & 7 & 2 & 6 & 0 \\
\hline Psychiatric diagnosis & $\begin{array}{l}\text { Patanofol } \\
\text { schizophrenia }\end{array}$ & $\begin{array}{l}\text { Cyctond } \\
\text { peychosis }\end{array}$ & $\begin{array}{l}\text { Gycloid } \\
\text { psychosits }\end{array}$ & $\begin{array}{l}\text { Bipolar } \\
\text { alfective } \\
\text { disonder }\end{array}$ & $\begin{array}{l}\text { Cychoid } \\
\text { psychosis }\end{array}$ & $\begin{array}{l}\text { Cychoid } \\
\text { peychosis }\end{array}$ \\
\hline
\end{tabular}


The third report concerned a woman (with del $15 q 11 \mathrm{q} 13$ ) who was 28 years old at the onset of the psychotic illness. She had received some advice from a clinical psychologist for obsessional features over the 6 months preceding the onset of the psychotic disorder. The subject came home from her day centre, sat down to watch TV and suddenly burst into tears saying 'my kittens have died'. The family did not have any kittens. A few minutes later, she became convinced that the heating system was about to explode. The subject was seen by her general practitioner, who referred her to an accident and emergency department for an investigation of a presumed plysical illness. No physical abnormality was found and she was transferred to a psychiatric hospital. The subject refused to get into a bed near an electrical socket because she was convinced it would catch fire. She received no regular medication and was discharged after 2 weeks. The subject was subsequently referred to a psychiatrist specializing in learning disability because other abnomal beliefs and was prescribed thioridazine.

Clarke (1993) suggested that these psychoses occurring in people with PWS most closely resembled the cycloid psychoses described by Perris and others (e.g. Perris 1988) because the sudden onset and polymorphous nature of the symptoms was striking. Ewald et al. (1994) suggested an association between PWS and manic depressive disorder. These suggestions are not necessarily incompatible: cycloid psychoses may be a subset of affective disorders (Bertelsen et al. 1977; Brockington et al. 1982).

A 20-year-old woman with PWS with a persistent delusional disorder was subsequently reported, and issues surrounding the classification of psychoses associated with PWS were explored in a publication documenting the clinical features (Clarke et al. 1995). This patient (with del 15q11 q13) differed from the other cases described in that the psychosis was largely monosymptomatic and was persistent. The principle feature was an abnormal belief that her parents were being informed, in a critical way, of her actions and movements.

Whittaker ef al. (1997) reported a 17-year-old woman with PWS who developed a psychosis chancterized by a rapid onset and clinical features which included anxiety, perplexity, tearfulness, food-refusal, delusions and hallucinations, with a rapid resolution following antipsychotic treatment. The authors commented on the marked similarity to cases reported by Clarke (1993), notably the lack of any precipitating factor, and the presence of both affective and paranoid symptomatology with a rapid onset and resolution. Verhoeven ef at. (1998) described six men with PWS and cycloid psychoses.

Other reports in the literature have mentioned psychotic features in association with PWS, but the focus of such reports has usually been on some other aspect of the syndrome and the clinical features of the psychoses are not described in detail. Jerome (1993) described a 31-year-old woman with PWS and a rapid cycling bipolar disorder treated with fluoxetine and lithium. A PWS case reported by Tu 
et al. (1992) may also have had a bipolar disorder. Bhate et al. (1989) described a 36year-old woman with mosaicism for del $15 \mathrm{q} 11$ who had hypothyroidism (the focus of the above paper), and had undergone hospital admissions because of 'extreme restlessness, psychosis and disruptive behaviour which requires phenothiazine medication', the psychosis not being related to the hypothyroidism. Bray et al. (1982) reported that two out of their series of 40 people with PWS had hallucinations and withdrawal and required admission". Whitman \& Accardo (1987) used the Survey Diagnostic Instrument a 134-item questionnaire screening for DSM-III-criteria-based diagnoses, with a sample of young people with PWS in the USA. These authors found that over half of the 35 adolescents with PWS surveyed had a definite or probable DSM-III diagnosis, compared with $18.7 \%$ of the general population. The diagnoses were predominantly of neurotic disorders, although suspiciousness, 'strange' ideation, and auditory or visual hallucinations were also reported. Twelve out of the 35 subjects had "occasional auditory hallucinations,' and abnormal suspiciousness and suicidal ideation were also reported. In the second paper in this series, Clarke (1998) describes a preliminary study of the prevalence of psychotic symptoms reported by carers of people with PWS using the Psychopathology Assessment Schedule for Adults with Developmental Disability (PAS-ADD) checklist.

\section{Method}

Cases of PWS associated with psychoses which had not been previously reported in the literature were assessed by contact with the psychiatrist who had managed the episode of psychosis, case-note review and interview of the affected person and their carer(s).

The expression of SNR PN and PAR-5 was investigated in the people with PWS and psychotic symptoms described because these two expressed sequences lie closest to the imprinting centre, and any disturbance in imprinting around this region may be related to the additional symptoms experienced by these individuals. A method for detecting the expression of SNRPN in blood samples by reverse transcriptase polymerase chain reaction (PCR) using expression of the WASP gene as a control has been described previously (Wevrick \& Francke 1996).

\section{Cytogenetic studies}

Chromosome studies were carried out at the 850 band level. Elongated prometaphase chromosomes were obtained by the addition of $10-4 \mathrm{M}$ ethidium bromide to the culture medium for $2 \mathrm{~h}$ prior to harvest. Harvesting and slide 
making were perfomed using standard methods, and the chromosome spreads were GTG banded.

\section{Molecular studies}

The DNA was extracted from EDTA-treated whole blood by standard methods. As parental samples were not available, the probands were genotyped using highly informative (CA) repeats at loci within $15 \mathrm{q} 11 \mathrm{q} 13$. It has been shown previously that the presence of a single band at D15\$128 demonstrates hemizygosity and is diagnostic for PWS (Andrew et al1997).

\section{Expression studies}

Venous blood samples in EDTA tubes were used within 24 h of phlebotomy for total RNA extraction using an RNeasy total RNA kit Quiagen Crawley, UK). Single-stranded cDNA was then synthesized from these samples using a first strand synthesis kit (Amersham Life Sciences, Amersham, UK).

Multiplex PCR was then performed on these DNAs using two sets of primers. The first set was designed to amplify a cDNA sequence from the SNRPN gene and the second to amplify a sequence from the DNA of an X-linked lymphocyte specific gene (WASP). This second PCR reaction acted as a control to ensure that the amplification system was working correctly. The two products were separated by electroptioresis on a $2 \%$ agarose gel and the respective sizes were determined by compatison with a DNA size ladder. The absence of an SNRPN amplification product in the presence of a WASP amplification product indicated a lack of expression of the SNRPN gene. If both amplification products were present, this indicated nomal expression of both genes. The expression of a further anonymous DNA fragment PAR -5 was also studied in a similar multiplex PCR system. Primers designed to amplify PAR-5 CDNA were used in conjunction with those from the WASP gene exactly as for SNRPN.

\section{Results}

Six people with PWS who had had psychotic symptoms and their carers agreed to the release of intormation for the present study. The clinical characteristics of the participants are summarized in Tables 1-4. Descriptions of the psychiatric and genetic abnomalities are given below.

The imprinting status of the UK subjects with PWS and psychoses was determined by comparison of the expression patterns found for SNRPN and PAR-5 with that of three control individuals, and a proband with Angelman syndrome. The 
SNRPN and the WASP primers were designed to generate products of 143 and 324 bp, respectively. In this system, none of the thee samples from the PWS probands amplitied a 143-bp product and only the 324 bp product was observed. Thus, there was no expression of the SNRPN gene. However, four unaffected control individuals and a proband with Angelman syndrome demonstrated both bands.

The PAR -5 amplification product was found to be approximately 165 bp in length in control subjects. Bands were obtained from both the PAR -5 and the WASP CDNAs in non-PWS control individuals and the patient with Angelman syndrome. A single WASP band was detected in two out of the three samples from PWS subjects, indicating a lack of expression of PAR-5. The third person with PWS showed no amplification at all in this system.

Case 1 (F)

Prader-Willi syndrome was diagnosed at the age of 8 years in a female subject. When the patient was 20 years old, she started to behave strangely and nade odd comments such as 'you are looking at what I am looking at' during car joumeys. She was said to have had a 'mental breakdown' at her adult training centre (ATC), standing immobile and unresponsive to other people. Her GP prescribed thioridazine. The subject was than seen by a psychiatrist specializing in learning disability, who reported that, after a holiday with the ATC, F had left behind a treasured radiocassette player and tapes, saying that "machines are evil'. She talked a lot about God and religion, and wanting to do things her own way. The subject eventually walked out of the ATC and was missing for several hours. She was found by a vicar in the grounds of a local church, standing immobile by the gate and refusing to communicate. F eventually left when her brothet arrived and talked to her. She was prescribed haloperidol $(10 \mathrm{mg})$, but suffered severe Parkinsonian symptoms. The subject was subsequently prescribed thioridazine (which resulted in sedation) and sulpiride. The clinical features noted at the time included suspiciousness and bizarre behaviour. In 1991, she was admitted to hospital to help her to lose weight and psychotic symptoms were not documented. In 1993, Fbecame 'clearly psychotic', going to a local river to swim and praying at the side of the river. She was unable to swim. Her sulpiride was increased. Two years later, she had another episode of psychosis, with features including restessness and agitation, removing her clothes inappropriately, and screaming and focusing on bizarre matters, often from the past'. The subject was eventually detained under Section 3 of the Mental Health Act. The episode seems to have lasted for about 6 months, at which time her 'Section' lapsed.

Cytogenetic studies showed a microscopically visible deletion in $15 \mathrm{q} 1 \mathrm{lq} 13$. DNA studies demonstrated only a single band at D15S128. Surprisingly, two 
bands were seen at GABR AB3 and three at D15\$113. Both of the latter loci lie not in the PWS but in the Angelman syndrome chromosome region. Expression studies showed that F did not express either SNRPN or PAR-5.

\section{Case 2(G)}

On returning to the family home from a residential placement at the age of 21 years, $G$ was noted to be behaving oddly and apparently experiencing hallucinations. She was admitted to hospital. A sample of speech recorded verbatim in the case notes reads as follows:

'Frightened by a bear, a polar bear. He was not standing, he was crawling almost. It was horrible. It was a spirit. I could not protect myself. My parents tried to help by allowing me to sleep between them but this did not help. I had a lot of fear. I was frightened of the guns and the swords. I also put a lot of father"s gun cartridges in my toilet bag. The bear was after me. Wherever I was, he was there. It's nothing I'd done wrong."

The subject refused to sleep alone, put out food for a 'spirit', talked about the polar bear, which she said had green feet, and in the middle of winter, left the hospital and was found standing in a field. She was discharged after 6 weeks. Further episodes recurred approximately every 6 months with a sudden onset of irrational fears. The subject was eventually treated with trifluoperazine in doses up to $12 \mathrm{mg}$ daily, and more recently, with paroxetine $(20 \mathrm{mg}$ daily, the dose of trifluoperazine being reduced to $1 \mathrm{mg}$ daily). She also receives carbamazepine ( $100 \mathrm{mg}$ daily). Her mental state has been more stable, and $G$ has been more alert since the introduction of paroxetine and the reduction of trifluoperazine. The subject did not have a cytogenetically visible deletion in $15 \mathrm{q} 11 \mathrm{q} 13$. However, $\mathrm{G}$ did have a single $\mathrm{CA}$ repeat band at D 15S128 and also at D 15S11. Expression studies showed only the WASP band, indicating that she did not express either SNRPN or PAR-5.

\section{Case $3(H)$}

This female subject first developed psychotic symptoms at the age of 14 years, whilst on holiday with her parents. She acted strangely, staring into space and moving more slowly than usual'. The subject talked in monosyllables and appeared disorientated. On her return home, $\mathrm{H}$ was admitted to hospital for a short time and received a dragnosis of an acute confusional state which had resolved. At the age of 15 , she became withdrawn and had disordered speech. The subject believed that people were against her, and said that she had been bitten by a scorpion and by a police dog. She was prescribed trifluoperazine $2 \mathrm{mg}$ daily, made a good recovery and the antipsychotic was withdrawn after 2 months. At the age of 17 years, $\mathrm{H}$ became withdrawn and detached and talked about pigeons "whistling 
and walking on the roof, which would not have been possible. She was trated with sulpiride $400 \mathrm{mg}$ twice daily. She contimued to have abnormal beliefs; for example, that criminals were hiding in her room. At the age of 18 years, $\mathrm{H}$ developed ideas of persecution by "the witch from Snow White' She apparently experienced visual hallucinations of a woman who lay on her bed and crumpled her bed clothes. The subject became withdrawn and tearful, and markedly slow in her movements during the morning, raising the suspicion that she had a depressive disorder. She was admitted to a psychiatric treatment facility for people with learning disability, and described a number of abnormal beliefs. These included the belief that the 'Black Witch from Snow White' was trying to poison her with apples, with the help of the police. Her symptoms slowly resolved following an increase in sulpiride to $600 \mathrm{mg}$ twice daily. The subject had two further episodes of psychosis, apparently in response to life stresses. Her mental state has stabilized following treatment with risperidone ( $5 \mathrm{mg}$ daily) and carbamazepine (200 $\mathrm{mg}$ three times daily). Electroencephalography and a computed tomography brain scan showed no abnormalities.

Previous cytogenetic studies showed that this proband carried a maternally derived translocation of chromosomes 13 and $15-46 \times X(t 13 ; 15)(\mathrm{p} 12 ; \mathrm{q} 22.1)$. In view of this finding, and because a small venous blood sample was obtained, only expression studies were carried out. No expression of either SNRPN or PAR-5 was detected.

Case 4 (I)

This male subject became mentally unwell after a weekend holiday in France and shortly after receiving the second of two intramuscular injections of testosterone (50 mg monthly). On the ferry returning to the UK, he became aggressive, saying that he hated his father and his sister. He told people to leave him alone, seemed very suspicious and said that people were against him. The subject commented that, 'nothing looks right, everything is whizzing round in my head'. He talked almost continuously, and was seen by a doctor and prescribed trifluoperazine on leaving the ferry. The subject was seen by his general practitioner, who prescribed diazepam, and was seen the next day at a general hospital. He was assessed and transferred to the psychiatric unit within the hospital. On admission, he was talking non-stop, saying 'it' was his father's fault and that he did not believe the building was a hospital. The subject was prescribed antipsychotic medication, recovered and was discharged after 3 weeks. Currently, I receives chlorpromazine (50 $\mathrm{mg}$ at night), procyclidine ( $5 \mathrm{mg}$ twice daily) and trifluoperazine $7 \mathrm{mg}$ three times daily). His level of functioning is believed to have declined following his illness, but this may have partly been because his mother was diagnosed as suffering 
from a potentially life-threatening illness a few months after he was discharged from hospital.

Previous genetic studies had demonstrated the presence of matemal uniparental disomy. Venesection was not possible, and detailed genetic studies could not be carried out.

\section{Case $5(\mathrm{~J})$}

One day, when he was 12 years old, J was hit by a schoolmate. He became restless, woke during the night and paced around. He emptied a number of beer bottles in the house into glasses, put a piece of apple into each glass and added pepper. $\mathrm{He}$ then went to his mother's bedroom, saying, 'They won't hit me any more. I shall poison them all." The same pattern of behaviour was repeated on the two successive nights. The subject was admitted to a paediatric unit to exclude an organic cause. Computed tomography scanning showed asymmetric subcortical atrophy. An electroencephalogram and ophthalmological examinations found no abnormality. The unusual remarks and agitation, with periods of apathy, continued for about 10 days. His behaviour gradually reverted to normal. No medication was given. Three weeks later, I was pyrexial $(38.6 \mathrm{C}$ ) and he stayed in bed for a day. The next day, the subject attended school, but was sent home by the school nurse, who reported that, 'He is apathetic, and sits in the class like a statue.' The next morning, J was anxious and agitated and told his mother that he had dreamed about ghosts who wanted to kill him. He again recovered without medication being given.

At the age of 14 years, J returned home from a summer camp and told his mother, 'I was hit with a belt. I want to be a soldier and travel far away.' He was agitated and threw all his toys in a bin. During the night, he repeated short phrases such as 'the door is dirty" and "I am going to Mexico". The subject hit his siblings and threw chairs around. The following day, he became withdrawn and refused to eat or drink. The subject lost $2 \mathrm{~kg}$ in a week. J was prescribed haloperidol ( $3 \mathrm{mg}$ ) and made a good recovery. He had extrapyramidal adverse effects and the medication was changed to pimozide ( $1 \mathrm{mg}$ daily). Chromosome examination at the age of 15 years showed a $15 \mathrm{q} 11 \mathrm{q} 13$ deletion. At the age of 22 years, J had a further psychotic episode with incoherent speech and bizarre behaviours such as putting his shoes and suitcase into a washing machine. His mental state improved following treatment with haloperidol, sulpiride and fluoxetine.

Case $6(K)$

This subject became mentally unwell at the age of 16 years after a day at school. She came home and told her mother that wolves had chased her and wanted to kill her. 
Her mother described her as 'chaotic, anxious, agitated, disturbed, aggressive, stubborn and uncontrollable'. The subject was treated with haloperidol and her mental state settled. Her parents were reluctant to continue the medication and stopped it. One month later, $\mathrm{K}$ had a similar episode with a sudden onset of anxiety, agitation, and auditory and visual hallucinations. Haloperidol was prescribed and she improved over the following 2 days. Two months later, when the dose of haloperidol was reduced, a further episode of agitation, bizarre behaviour and incoherent speech occurred. K had another episode at the age of 17 with aggressive behaviour, agitation and incoherent speech. She refused food and fluids, and was admitted to a paediatric unit. She was agitated and paced around the unit. The subject had a restless night. The following day, $\mathrm{K}$ became withdrawn. She shivered and refused to speak. The agitation, disturbed sleep pattern and unusual remarks continued for a week, after which she made a good recovery and was discharged taking no medication. Over the following 7 years, she had several similar episodes with sudden onset, agitation, anxiety, incoherent speech, social withdrawal, auditory hallucinations and sleep disturbance. One nurse remembered $\mathrm{K}$, telling her, 'Watch out for the old toilet! You will fall in it and drown!' and 'The old Belgians will kill me.'

\section{Discussion}

Five out of the six cases described had genetically verifiable PWS and there is litcle doubt about the diagnosis in the sixth case. Detailed genetic testing was carried out on three out of the four people with PWS from the UK. Although expression of both SNRPN and PAR-5 was detected in three control individuals and in a patient with Angelman syndrome, none of the three PWS probands with psychoses expressed either locus. As these two loci lie very close to the inprinting centre, this suggests that their psychotic symptoms are not the result of a global failure in the imprinting process. SNRPN is one of the few genes isolated from the PWS chromosome region. It remains a likely candidate gene, although unlike Angelman syndrome, PWS is probably a contiguous gene syndrome. In case I (F), the deletion, although large enough to be visible microscopically, did not extend into the Angelman chromosome region and the presence of extra bands at D15S113 indicates that a rearrangement may have occurred in the $1511 \mathrm{~g} 13$ region. Although case $2(\mathrm{G})$ clid not have a cytogenetically visible deletion, the hemizygosity shown at D $15 \$ 128$ and at D $15 \$ 11$ indicates that she most probably has a small deletion. She may possibly have matemal isodisomy, which is rarer than heterodisomy as a cause of PWS. Case G also had absence of expression of both the expressed sequence tags located in the PWS chromosome region. The finding of a translocated chromosome 15 in case 3 (H) also suggests matermal uniparental 
disomy, but in this case, maternal heterodisomy. She also had no expression ofloci in the PWS critical region. Thus, these three probands with psychotic symptoms probably demonstrate all three types of molecular abnormality usually associated with PWS. The only common finding is an inability to express sequences within the PWS critical region.

Taken in isolation, this series of case reports could be dismissed as descriptions of people with a disorder (PWS) associated with intellectual disability who have atypical psychotic disorders. Intellectual disability is itself associated with an increased risk for psychotic disorders (Corbett 1979; Lund 1985), although some of this increase in risk may be accounted for by specific associations, such as that postulated between PWS and psychosis. There is also, for example, evidence of a vulnerability to psychoses associated with the velo-cardio-facial (Shprintzen) syndrome (Papolos et al 1996).

However, together with the other papers in this issue (Clarke 1998; Verhoeven ot al. 1998) and the other reports summarized in the introduction, the present study strongly suggests that PWS is associated with a vulnerability to psychotic symptoms in adult life. Other forms of psychiatric disorder may also be more common in association with PWS. Bartolucci \& Younger (1994) suggested that such disorders could be divided into exaggerations of behaviours commonly seen in association with the syndrome (the behavioural phenotype), florid psychotic states and "lethargic- refusal" states. Dykens et al. (1996) found a high prevalence of obsessive-compulsive symptoms associated with PWS.

Prader-Willi syndrome may be directly implicated in the genesis of psychotic symptoms (e.g. as a result of a protein deficiency resulting from the genetic abnormality) or it may be indirectly related (e,g. through the influence of neuropsychological abnormalities associated with PWS, such as auditory information processing deficits). Many of the clinical features of PWS (e.g. appetite and slecp abnormalities, mood and temperature instability, and self-injury) suggest an abnomality of serotonin or neuropeptide function in the central nervous system which is probably of hypothatamic origin. It is also possible that the psychotic symptoms result from a transient metabolic abnormality. In this context, it is notable that psychotic symptoms were on one occasion related to pyrexia in one case reported in the present paper. The present authors are aware of one other case (never reported in the literature) in which a high temperature resulted in what seems to have been a transient psychosis in a young man with PWS.

If the vulnerability of adults with PWS to psychotic and obsessional symptoms is confirmed, the cause of such an association will merit intensive study in view of ats importance both to people with PWS, and to our understanding of the causation of functional psychoses and obsessional states. Information about treatment response will also be valuable in shedding light on possible aetiological mechanisms. 


\section{Acknowledgements}

We are grateful to-the people with PWS and their carers, who made this study possible, and to Dr C. Bainton, who allowed us access to records.

\section{References}

Andrew N., Lambert J. \& Cooke A. (1997) D15\$128 in diagnosis of Prader-Willi and Angelman syndromes. Joumal of Medial Genctio 34, 874.

Barcolucci G. \& Younger J. (1994) Tentative classification of neuropsychiatric disturbances in Prader-Willi syndrome. Joumal of Intellectual Disability Researh 38, 621-9.

Bertelsen A., Harvald B. \& Hauge M. (1977) A Danish twin study of manic-depressive disorders. British Jowrual of Psythiatry 130, 330-51.

Bhate M. S., Robertson P. E., Davison E. V. \& Brummitt J. A. (1989) Prader-Willi syndrone with hypothyroidism. Journal of Mentai Deficieny Researd 33, 235-44.

Bray G. A., Dahms W. T., Swerdloff R. S., Fisher R. H., Atkinson R. L. \& Carrell R. E. (1982) The Prader-Willi syndrome: a study of 40 patients and a review of the literature. Medicime $62,59-80$.

Brockington I. F., Perris C., Kendell R. E., Hiflier V. E. \& Wainwright S. (1982) The course and outcome of cycloid psychosis. Psychological Medicine 12, 97-105.

Clarke D. J. (1993) Prader-Willi syndrome and psychoses. British Jourmal of Psyihiarry 163 , $680-4$.

Clarke D. J. (1998) Prader-Willi syndrome and psychotic symptoms: 2. A preliminary study of prevalence using the Psychopathology Assessment Schedule for Adults with Developmental Disability checklist. Joumal of Intellectual Disability Researih 42, 451-454.

Clarke D. J., Boer H., Chung M. C., Sturmey P. \& Webb T. (1996) Maladaptive behaviour in Prader-Willi syndrome in adult life. Joumal of Intellectwal Disadility Researd 40,159-65.

Clarke D. J., Waters J. \& Corbett J. A. (1989) Adults with Prader-Willi syndrome: abnomalities of sleep and behaviour. Joumal of the Royal Socicty of Medicituc 82, 21-4.

Clarke D. J., Webb T. \& Bachmann-Clarkej. P. (1995) Prader-Willi syndrone and psychotic symptoms: a further case. Irish foumal of Psychological Mcdicine. 12, 27-9.

Corbett J. A. (1979) Psychiatric morbidity and mental retardation. In: Pspchiatric Ilhuess awd Mental Handicap (ed. P. Snaith \& F.E James), pp. 11-25. Headley, Ashford.

Dykens E. M., Leckman J. F. \& Cassidy S. B. (1996) Obsessions and compulsions in Prader-Willi syndrome. Jostrut of Child Psychology and Psychianry 37, 995-1002.

Ewald E., Mors O., Flint T. \& Kruse T. A. (1994) Linkage analysis between manic depressive illness and PWS $15 q 1113$ deletion. Human Heredity 44, 287-94.

Greenswag L. R. (1987) Adults with Prader-Willi syndrome: a survey of 232 cases. Deyetopmental Medicine and Child Neuralogy 29, 637-48.

Jerome L. (1993) Prader-Willi syndrome and bipolar illness. Jounval of the American Acadeny of Child and Adolessent Psychiatry 32, 876-7. 
Kollrack H. W. \& Wolff D. (1966) Paranoid-halluzinatonische Psychose bei Prader-LabhartWilli-Fanconi syndrome. Acta Padopsychatrica 33,309-14.

Lund J. (1985) Prevalence of psychiatric morbidity in mentally retarded. Aca Psyairica Scandiravia $72,563-70$.

Papolos D. F., Faedda G. L., Vest S., Goldberg R., Morrow B., Kucherlapati R. \& Shprintzen R. J. (1996) Bipolar spectrum disorders in patients diagnosed with velo-cardiofacial syndrome: does a hemizygous deletion of chromosome 22 qil result in bipolar affective disorder? Amcrion Joumal of Psydiatry 153,1541-7.

Perris C. (1988) The conceptof cycloid psychotic disorder. Psychiatric Developwents 1, 375-6.

Prader A., Labhart A. \& Willi H. (1956) En Syndrom von Adipositas, Kleinwuchs, Kryptorchismus und Olgophremic nach myatonieartigem Zustand im Neugeborenalter. Shweizerische Medizinische Wodhensdriff 86, 1260-1.

Robinson W. P., Bottam A., Yagang X., Balakrishnan J., Binkert F., Machler M. Prader A. \& Schinzel A. (1991) Molecular, cytogenetic and clinical investigations of Prader-Willi syndrome patients. Amerian Jownal of Huwan Genetics 49, 1219-34.

Sutcliffe J. S. Nakao M., Christian S., Orstavik K. H., Tommerup N., Ledbetter D. H. \& Beaudet A. L. (1994) Deletions of a differentially nethylated CPG island at the SNRPN gene define a putative imprinting control region. Nature Genetics 8, 52-58.

Tu J.-B., Hartridge C. \& Izawa J. (1992) Psychophamnacogenetic aspects of Prader-Willi syndrome. Joumal of the American Acadewy of Chitd and Adolescent Psychary 31, 1137-40.

Verhoeven W. M. A., Curfs L. M. G. \& Tuinier S. (1998) Prader-Willi syndrome and cycloid psychoses. Joumal of Intellectual Disability Researh 42, 455-462.

Wevrick R. \& Francke U. (1996) Diagnostic test for the Prader-Willi syndrome by SNRPN expression in blood. Lancet 348, 1068-9.

Wevrick R., Kerns J. A. \& Francke U. (1994) Identification of a novel paternally expressed. gene in the Prader-Willi syndrome region. Howan Molewar Genetio 3, 1877-82.

Whitman B. Y. G Accardo P. (1987) Emotional symptoms in Prader-Willi syndrome adolescents. American Jowmal of Medical Generics 28,897-905.

Whittaked. F., Cooper C., Haningron R. C. k Price D. A. (1997) Prader-Will syndrome and acutio psychosis. Whemational Joumal of Psydiatry in Clinial Pratice 1, 217-19. 
Chapter 6b

\section{Psychotic illness in people with Prader-Willi syndrome due to chromosome 15 maternal uniparental disomy}

Published as:

Boer H., Holland A., Whittington J., Butler J., Webb T. \& Clarke D. (2002) Psychotic illness in people with Prader-Willi syndrome due to chromosome 15 maternal uniparental disomy. Lancet 359, 135-6. 
In a population-based study of Prader-Willi syndrome (PWS), we investigated the relation between genetic subtypes of the syndrome and psychiatric morbidity. Of 25 patients aged 18 years or older, seven (28\%) had severe affective disorder with psychotic features, with a mean age of onset of 26 years (SD 5.9). The seven people affected, all aged 28 years or older, included all five with disomies of chromosome 15, one with a deletion in this chromosome, and one with an imprinting centre mutation in the same chromosome. We postulate that in PWS, an abnormal pattern of expression of a sex-specific imprinted gene on chromosome 15 is associated with psychotic illness in early adult life.

Prader-Willi syndrome (PWS) is a genetically determined developmental disorder. The main features of the syndrome are neonatal hypotonia, hypogonadism, extreme overeating, short stature, characteristic physical appearance, and mild learning disabilities. Skin picking, obsessive behaviours, a tendency to have temper tantrums, and sleep abnormalities have also been reported (Holmet al. 1993). Previous case reports have suggested that rates of affective or cycloid psychoses might be increased in PWS (Verhoeven et al, 1998). The two main genetic causes are deletions in patemal chromosome 15 (q11-13) and maternal disomy of chromosome 15 , with chromosomal translocations and mutations of an imprinting centre accounting for less than $5 \%$ of cases (Wevrick and Francke, 1996). Although candidate genes at 15q11-13 that have been maternally imprinted have been identified, the exact gene (or genes) that gives rise to the PWS phenotype is unknown.

In this epidemiological study, we aimed to investigate the extent of phenotypic variation in PWS, to establish the prevalence of physical and psychiatric morbidity, and to compare the phenotypes of those with different genotypes. All individuals with possible PWS who were living in the old Anglia and Oxford health region of the UK (population 5 million) were identified through several sources (Whittington ef al. 1991). Established informant and participant-based assessments were used to determine the extent of diagnostic and other physical and behaviourai characteristics. In patients whose records were available, or when consent for venepuncture had been given, diagnosis of PWS was confirmed with the methylation pattern of small nuclear ribonucleoprotein N.3 Molecular and cytogenetic techniques were then used to ascertain the genetic subtype of PWS.

To investigate rates of psychiatric morbidity, all people with PWS and a history of psychiatric treatment, all those presently receiving psychotropic drugs, and any who scored positively on a brief mental health questionnaire, were seen by a psychiatrist $(\mathrm{HB})$ who was skilled in assessment of people with leaming disabilities. Information on mental health was obtained from the participant, informants, and from the participant's health records. Case summaries were prepared, and the assessing psychiatrist and two other expert psychiatrists (AH and DC), who were unaware of genetic status, made independent diagnoses. 
92 people living in one health region of the UK who had been identified as possibly having PWS agreed to participate. 65 met clinical criteria, and for 61 , this was confinned in a blood test (blood was not available for four). The four without genetic confirmation were not investigated further. In 54 patients the exact genetic type of PWS was established, all of whom were tested for psychiatric illness. Since none of those aged younger than 18 years tested positively for serious psychiatric illness, we restricted our analysis to the 25 adults who had a PWS genotype. At the initial interview, ten of these $25(40 \%)$ were referred with possible psychiatric illness and therefore had a detailed psychiatric assessment. All these individuals were over 28 years, therefore, to check whether the interview had identified all cases, we assessed all but one of the remaining patients aged 28 years or older $(n=5)$ (one person refused). In total, 15 of $25(60 \%)$ adults with confimed PWS had a detailed psychiatric assessment. One further patient with a psychatric illness was found on the detailed assessment. Her lack of speech was probably the reason she was not picked up in the screening interview. None of the remaining unscreened patients had poor speech and thus it is unlikely that a severe psychiatric illness was missed.

Table 1 shows the characteristics, symptoms, and diagnoses of the patients. Table 2 shows the results of the assessments, and other details of all 15 adults. Seven of these $15(47 \%)$ were independently assessed as having a major affective or psychotic illness by all three psychiatrists. In these patients, the characteristic features were a combination of a mood disorder and evidence of psychotic symptoms. The rate of serious psychiatric illness in adults with PWS was therefore $28 \%$ (seven of 25). Although the patient with the imprinting centre mutation had not reported having specific abnormal mental experiences, he undoubtedly had a severe mood disorder that included suicidal ideation. He was therefore included as one of the seven parients with major psychiatric illness. One of $13(8 \%)$ adults with deletions had psychotic illness, compared with five of eight (62\%) with disomy (risk ratio $8 \cdot 125 ; 95 \% \mathrm{CI} 1 \cdot 15-57 \cdot 6)$. For those aged 28 years or older, five of five (100\%) patients with disomy, and one of nine $(11 \%$ ) with a deletion had psychotic illness $(9.0 ; 1.42-57 \cdot 1)$.

The $8 \%$ rate of psychotic illness in adults with chromosome 15 deletions only just exceeds the $5 \%$ reported for affective disorder in adults with learning disabilities (Cooper \& Collacott, 1996). The 100\% rate of psychotic illness in older adults with chromosome 15 disomy cannot therefore be due to having only PWS and associated learning disabilities. If we assume the base rate of psychotic illness in those with PWS due to a deletion to be at most $10 \%$, the odds of all five older people with disomy being psychotic by chance would be one in 100000 .

Case reports (Verhoeven et al. 1998) support this hypothesis. Verhoeven and colleagues (1998) reported that of six people with PWS and an affective disorder or cycloid psychosis, three of the four with known genetic abnormality had 
Table 1. Summary of characteristics, symptoms, and diagnoses of people with Prader-Willa syndrome who had a detaited psychiatric assessment

\begin{tabular}{ll}
\hline & Patients (n=15) \\
\hline Characteristics & $38 \cdot 5(5 \cdot 7)$ \\
Age (mean [SD], years) & 8 \\
Sex Men $\quad$ Women & 7 \\
Body-mass index (mean [SD], $\mathrm{kg} / \mathrm{m} 2)$ & $38 \cdot 0(13 \cdot 3)$ \\
Age at anset (mean [SD], years) & $30 \cdot 0(8 \cdot 4)$ \\
Drugs* & \\
Antipsychotics & $7(47 \%)$ \\
Mood stabilisers & $5(33 \%)$ \\
Antidepressants & $9(60 \%)$ \\
None & $4(27 \%)$ \\
& \\
Genetic abnormality & \\
Del 15q11q13† & $9(60 \%)$ \\
Imprinting mutationf & $1(7 \%)$ \\
Matemal heterodisomy & $5(33 \%)$ \\
& \\
Psychotic symptoms & \\
Paranoid delusions alone & $1(7 \%)$ \\
Paranoid delusions and hallucinations & $5(33 \%)$ \\
None & $9(60 \%)$ \\
Clinical diagnosis &
\end{tabular}

Values are number $(\%)$ unless otherwise indicated. *Some patients were taking more than one type of drug. $\dagger$ three were heterozygous at 0155165 . \$ Paternal contribution at 015511,0155210 , and 015597 , but not at D155128. \$In psychiatric group.

uniparental disomy. We therefore postulate that anabnomal pattern of expression of a maternally or paternally imprinted gene on chromosome 15 might lead to development of psychotic illness in PWS in adulthood. If this hypothesis is correct, allelic variation of this imprinted gene could be one cause of genetic vulnerability to psychotic illness in the general population. 
Table 2. ICD10 diagnoses for 15 people with Prader-Willi syndrome who had a detailed psychiatric asssessment

\begin{tabular}{|c|c|c|c|c|c|c|c|c|c|}
\hline Case & Gender & Clinical Diagnosis & Age & Med & $\begin{array}{l}\text { Age of } \\
\text { Onset }\end{array}$ & 10 & $B M I$ & $\begin{array}{l}\text { Genetic } \\
\text { abnormality }\end{array}$ & $\begin{array}{l}\text { Psychotic } \\
\text { symptoms }\end{array}$ \\
\hline 1 & $\mathrm{~F}$ & $\begin{array}{l}\text { Depressive } \\
\text { psychosis }\end{array}$ & 47 & $a, b, c$ & 21 & 71 & 31 & Del15q1.193 & $\begin{array}{l}\text { Paranoid } \\
\text { delusions }\end{array}$ \\
\hline 2 & $M$ & $\begin{array}{l}\text { Bipolar affective } \\
\text { disorder }\end{array}$ & 29 & $a, b, c$ & 19 & 65 & 37 & $\begin{array}{l}\text { Imprinting } \\
\text { mutation }\end{array}$ & Nome \\
\hline 3 & $F$ & Depressive episade & 33 & c & 32 & 78 & 74 & Del15q11q13 & Hane \\
\hline 4 & M & Psychotic disorder & 41 & $a, b, c$ & 28 & 62 & 43 & $\begin{array}{l}\text { Maternal } \\
\text { heterodisony }\end{array}$ & $\begin{array}{l}\text { Paranoid } \\
\text { delusions and } \\
\text { hallucinations }\end{array}$ \\
\hline 5 & $M$ & $\begin{array}{l}\text { Behavioutal } \\
\text { problems }\end{array}$ & 33 & $a, b$ & & 53 & 24 & Del15q11q13 & None \\
\hline 6 & $\mathrm{~F}$ & $\begin{array}{l}\text { Bipolar affective } \\
\text { disorder }\end{array}$ & 46 & a & 29 & 59 & 34 & $\begin{array}{l}\text { Maternal } \\
\text { heterodisomy }\end{array}$ & $\begin{array}{l}\text { Paranoid } \\
\text { delusions and } \\
\text { hallucinations }\end{array}$ \\
\hline 7 & $\mathrm{~F}$ & $\begin{array}{l}\text { Depressive } \\
\text { psychosis }\end{array}$ & 39 & c & 22 & 70 & 29 & $\begin{array}{l}\text { Maternal } \\
\text { heterodisomy }\end{array}$ & $\begin{array}{l}\text { Paranoid } \\
\text { delusions and } \\
\text { hallucinations }\end{array}$ \\
\hline 8 & $M$ & $\begin{array}{l}\text { Mild depressive } \\
\text { episode }\end{array}$ & 39 & $c$ & 36 & 91 & 32 & Del15q11q13 & None \\
\hline 9 & $\mathrm{~F}$ & Psychatic disorder & 38 & $a, b, c$ & 36 & 73 & 29 & $\begin{array}{l}\text { Maternal } \\
\text { heterodisomy }\end{array}$ & $\begin{array}{l}\text { Paranoid } \\
\text { delusions and } \\
\text { hallucinations }\end{array}$ \\
\hline 10 & $M$ & $\begin{array}{l}\text { Past depressive } \\
\text { episode }\end{array}$ & 36 & & & 68 & 52 & Del15q11q13 & None \\
\hline 11 & $\mathrm{~F}$ & $\begin{array}{l}\text { Bipolar affective } \\
\text { disorder }\end{array}$ & 34 & $c$ & 29 & 48 & 26 & $\begin{array}{l}\text { Maternal } \\
\text { heterodisomy }\end{array}$ & $\begin{array}{l}\text { Paranoid } \\
\text { delusions and } \\
\text { hallucinations }\end{array}$ \\
\hline 12 & $M$ & None & 39 & & & 51 & $?$ & Del15q11q13 & Mone \\
\hline 13 & $F$ & None & 37 & & & 62 & 48 & Del15q11q13 & None \\
\hline 14 & $M$ & $\begin{array}{l}\text { Mild depressive } \\
\text { episode }\end{array}$ & 47 & $a, c$ & 47 & 61 & 42 & Del15q11q13 & None \\
\hline 15 & $\mathrm{~N}$ & None & 40 & & & 68 & 31 & Dellsq11q13 & None \\
\hline
\end{tabular}

$a=$ antipsychotic, $b=\operatorname{mood}$ stabiliser, $c=$ antidepressant.

$B M I=$ body mass index, $10=$ Intelligence quotient

No 2 paternal contribution at 015511,0155210 , and D15597, but not at 0155128 .

Nos 12,13 and 14 heterozygous at D155165

\section{Acknowledgements}

This study was funded by the Wellcome Trust and the UK PWS Association. The sponsors had no role in the study design; data collection; analysis or interpretation; or in the writing of the paper. We thank the UK PWS Association for their help in identification of participants, and all participants with PWS, their families, and other carers for their very substantial help. 


\section{References}

Cooper S.A. \& Collacott R.A. (1996) Depressive episodes in adults with leaming disabilities. Irish Joumal of Psychologial Medicine 13, 105-13.

Holm V.A., Cassidy S.B., Butler M.G., Hanchett J.M., Greenswag L.R., Whitman B.Y. \& Grewberg F. (1993) Prader-Willi syndrome: consensus diagnostic criteria. Pediatrics 91. $398-402$.

Verhoeven W.M.A. Curf L.M.G. \& Tuinier S. (1998) Prader-Willi syndrome and cycloid psychoses. Journal of Intellecual Disability Research 42, 455-62.

Wevick R \& Francke U. (1996) Diagnostic tests for the Prader-Willisyndrome by SNURPN expression in blood. Lance $348,1068-9$.

Whittington J.E., Holland A.J., Webb T., Butler J.V., Clarke D.J. \& Boer H. (2001) Population prevalence and estimated birth incidence and mortality rate for people with Prader-Willi Syndrome in one UK Health Region. Journal of Medial Genetics 38, 792-8. 
Chapter $7 a$

\section{Differences in behavioural phenotype between parental deletion and maternal uniparental disomy in Prader-Willi syndrome}

Submitted as:

Stauder J.E., Boer H., Gerits R.H.A., Tummers J, Whittington J. \& Curls L.M.G. Differences in behavioural phenotype between parental deletion and maternal umiparental disomy in Prader-Willi syndrome. 


\section{Summary}

Background: Patemal deletion and matemal uniparental disomy are principal genetic abnormalities associated with Prader-Will syndrome (PWS). The present study is the first to address the behavioural phenotype of patemal deletion versus maternal uniparental disomy PWS subtypes in a cognitive psycho-physiological setup.

Methods: Behaviour and eventwelated brain activity (ERP) to a continuous performance response inhibition task (CPT-AX) was recorded in adults with paternal delecion PWS $(n=11)$, matemal uniparental disomy PWS $(n=11)$ and nomal controls $(n=11)$. The dependent behavioural variables of the CPT-AX task were reaction time and correct scores. For the ERPs the N200 and P300 components were included which are related to early modality-specific inhibition and hate general inhibition, respectively.

Results: The disomy group had fewer correct scores and increased reaction times as compared to the CPT-AX task than the control and deletion group. Both PWS subgroups differed significantly from the control group for the N200 amplitude. Only the control group showed the typical task modulation for the N200 amplitude. The amplitude of the P300 component was considerably smaller in the uniparental disomy group than in the deletion and control groups.

Conclusion: The ERP results suggest that early modality specific inhibition is impaired in both PW/S genetic subtypes. Late general inhibition is impaired in the uniparental disomy group only. Thus although the ERP data suggest a common impaiment in early visual inhibition processing, uniparental disomy and parental deletion genetic PWS subtypes clearly difer in their behavioural and brain activation phenotypes.

To date the Prader-Willi syndrome has been considered as a unitary syndrome despite the existence of three genetic subtypes. However, recently some anecdoal clinical differences have been reported between the two main genetic subtypes in Prader-Willi Syndrome. The present study seeks experimental confirmation to differentiate the two major experimentall subtypes in Prader-Willi Syndrome.

Prader-Willi Syndrome (PWS) is a genetically determined neurodevelopmental disorder associated with abnomal or absent expression of imprinted, paternally expressed genes at 15q11-q13 (Cassidy \& Schwartz 1998). The disorder is characterised by a number of clinicall features, e.g. severe neonatal hypotonia, failure to thrive, hypogonadism, cranio-facial abnomalities, short stature, and small hands and feet. From early childhood developmental delay and severe overeating behaviour become apparent (Cassidy et al. 1984; Holm et al. 1993; Whittington ef al. 2002; Holland et al. 2003). Most PWS individuals have a cognitive impaiment 
and function in the mild to moderate range of intellectual disability (Curfs et al. 1992; Dykens et al. 1992). A number of behaviours have been reported to be more common than expected in PWS such as stubbornness, temper-tantrums, obsessive-compulsive behaviour and intemalizing emotional problems (Holland ef al. 2003; Curfs et al.1992; Beardsmore et al. 1998; Clarke ot ol. 1996; Clarke of al. 2002; Dykens et al. 1999; Dykens of al. 1997). The incidence of PWS has been estimated at about 1:8000-1:20.000 live births (Burd ef al. 1990; Whittington ef al. 2001).

In PWS three genetic subtypes are involved. In approximately $70 \%$ of all PWS cases there is a small deletion in the paternally contributed chromosome 15 at the region 15q11-13 (deletion 15 genetic subtype). Around 28\% of the PWS patients inherit two maternally derived chromosomes 15 but no paternal chromosome 15 (uniparental disomy 15 genetic subtype). The remaining $2 \%-5 \%$ of patients with PWS have a chromosome-15 imprinting centre defect (Caissidy et al. 1998).

Cassidy et al. (1997) reported subtle phenotypic differences between paternal deletion and matemal disomy subgroups. The deletion group showed an increased frequency of hypopigmentation, a more typical facial appearance, a higher pain threshold, increased skin picking and more skill with jigsaw puzzles. Symons et al. (1999) investigated self-injurious behaviour and it appears that the deletion subgroup injures more body sites than the disomy group. Roof et al. (2000) found beside physical phenotypic differences that patients with maternal disomy had a higher average verbal intelligence (VIQ) in comparison to those with deletion, whereas there was no significant difference in average performance, or non-verbal intelligence (PIQ). Whittington et al. (2004) present compelling evidence that the disomy genetic subtype PWS individuals had better verbal skills than the deletion subgroup and that the deletion group had better visuo-spatial capacities than the disomy group. Finally, psychotic illness in Prader-Willi syndrome is mostly associated with the uniparental disomy 15 genetic subtype. (Boer et al. 2002; Vogels et al. 2003; Verhoeven et al. 2003).

Obsessive-compulsive behaviour has not been addressed extensively in PWS despite the often reported compulsive symptoms in this syndrome (Clarke of al. 2002; Dykens et al. 1996; Wigren et al. 2003). These compulsive symptoms may be caused by a deficit in inhibitory control (Pliszka et al. 2000). An often used experimental paradigm for addressing inhibitory control is the Go-Nogo task, in which subjects are instructed to respond to a certain category of stimuli but not to another. In the present study a so called CPT-AX task is used in which the subject is instructed to respond to the letter $X$ (the target) with a button press when it is preceded by the letter $A$ (the cue). If any other letter preceded the $X$ the subject is asked not to respond. Following the letter $A$ the subject must thus prepare a response in case the next letter is $\mathrm{X}$ (go- $\mathrm{AX}$ condition) or inhibit the prepared response if it is not (Nogo-Anot $X$ condition). 
During task peffomance we recorded the EEG continuously in order to obtain information on the inhibition processes that occur between the presentation of the stimulus and response of the participant. The Event Related Potential (ERP) can be extracted from the EEG by averaging the EEG traces that precede or follow a stimulus or event. The ERP is thus a manifestation of brain activity that arises in preparation for, or in reaction to, an event (Fabiani al. 2000). The ERP has several components that are typically classified in five ways: polarity (positive/negative peak); peak latency (point in time that the amplitude reaches maximum after stimulus onset); amplitude (maximum voltage recorded); topography (amplitude distribution across the scalp) and finally the sensitivity to the task manipulation. According to this logic "P300" refers to a positive peak with a latency of about $300 \mathrm{~ms}$. The latency indicates the timing of a specific cognitive process and the amplitude the degree of activation or intensity of that process. Stauder al. (2002) used ERPs to compare a group of patients with PWS to a control group. A visual- and auditory selective attention (oddball) task was presented in which participants were instructed to press a hand key to a target stimulus. Although both the PWS groups and the controls had good task performance, persons with PWS revealed clear P3 deflation in the visuall oddball task that was even more significant in the auditory oddball task. These results confimed suggestions in the literature that persons with PWS are more impaired in the auditory than visual modality and that they may have short-term memory impairment. The oddball tasks used. in the foregoing ERP study are relatively simple to perfom and evaluate functional domains such as selective attention and memory updating. A functional domain in which persons with PWS are specifically thought to be impaired is inhibitory control. The CPT-AX task is an experimental paradigm that has often been used for evaluating inhibitory control processes suclu as uesponse inhibition. The ERPS in such a CPT-AX task clearly differ between Gommals and Nogo-trials for a negative amplitude deflection around 200 to 300 ms after stimulus onset, coined the " $\mathrm{N} 200$ ' and for a positive going amplitude occurring between 300 to 500 ms, coined the 'P300'. Both the N200 and P300 components are increased in the Nogo as compared to the Go condition (Falkenstein of al. 1999). Jonkman ot al. (2003) found that nomal nine year old children had an adult N200 effect to a CPT-AX task despite the fact that they had more false alarms and higher impulsivity scores than adults. However, children Filed to show a fronto-central Nogo P300, suggesting that the P300 activity is more related to inhibition at the behavioural level than the $\mathbb{N} 200$. The latter suggestion is further corroborated by the finding that the $N 200$ is also present in children with inhibition disorders such as ADHD (Overtoom ef al. 1998).

The principal aim of the current Event Related Potential study is to present a CPT-AX task to persons with parental deletion PWS, matemal miparental disomy PWS and age matched controls in order to evaluate subte group differ- 
ences in inhibitory control. Given earlier reports of inhibitory problems in persons with PWS it can be expected that both groups differ from controls in the CPT-AX task, both at the behavioural level and for the ERP components N200 and P300. Although some of the above mentioned studies reported differences between the deletion and disomy PWS groups there is mot enough evidence to generate specific hypothesis of what PWS group differences can be expected in the current paradigm, except that the disomy group may have more mhibitory control problems than the deletion and control group. If differences between these PWS groups can be established it would be the first time that experimental behavioural and/or psychomphysiological findings confirm a differentiation between parental deletion en maternal uniparental disomy in Prader-Willi syndrome.

\section{METHODS}

\section{Participants}

Most EEG recordings $(n=15)$ were performed in and around Birmingham (UK), in several clinics and private houses. The remaining recordings $(n=7)$ were perfomed at the University of Maastricht and private houses across the Netherlands. Finally this resulted in recordings of 11 deletion and 11 disomy PWS patients. The gender and age matched controls $(n=11)$ were all measured in the Netherlands. In the deletion group there were 7 males and 4 fenales, the disomy group comprised 4 males and 7 fermales, and the control group included 6 males and 5 females. The average age for the groups were respectively was 26.7 years ( $\mathrm{sd}$ $=7.35$ ) for the deletion group, 27.7 years $(\mathrm{sd}=8.81$ ) for the disomy group and 27.3 years ( $s d=8.25$ ) for the controls. These ages do not significantly differ between groups. Handedness was evaluated using the Edinburgh Handedness Inventory (Oldfield 1971). The scoring in this test varies between - $100 \%$ to $100 \%$ where negative percentages indicate left-handedness and the positive percentages right-handedness. The control group had a mean scone of $68.94 \%( \pm 41.51)$, the disomy group of $81.06 \%( \pm 57.39)$ and the deletion group of $70.46 \%( \pm 58.38)$. The difference in handedness scores between the groups is not significant.

\section{Experimental reaction time task}

The stimuli of the CPT-AX task consist of a randomised sequence of eleven white capital letters $(A, B, C, D, E, F, G, H, J, L$, and $X)$, flanked by wo vertical lines, that are presented individually on a black background. The letters and vertical bars are $2 \mathrm{~cm}$ high and $1.5 \mathrm{~cm}$ wide and the participant was seated $60 \mathrm{~cm}$ in front of a 17 "VGA monitor, The participants were asked to press a hand button, as soon as 
possible, when the letter " $\mathrm{X}$ " followed the letter " $\mathrm{A}$ " (Go-condition $\mathrm{AX}$ ). In case another letter followed the letter 'A' (Nogo-condition Anot $X$ ), the response had to be suppressed. There were two practice sessions preceding the task. The first one consisted of a fixed sequence of 25 trials with $3 \mathrm{AX}$-sequences ( $\mathrm{Go}-$ condition 10), 2 AnotX-sequences (Nogo-condition 20), 2 not AX-sequences, and 11 not $A$ notX-sequences; the second practice session consisted of a fixed sequence of 15 trials with 2 AX-sequences (Go - condition), 1 AnotX-sequence (Nogo condition), 1 notAX-sequence, and 7 notAnotX sequences. The CPT-AX task consisted of four blocks of 124 letters each. Every block contained 12 AX-sequences, $12 \mathrm{~A}$ 's were part of a AnotX-sequence, $12 \mathrm{X}$ 's were part of a not $A X$-sequence and there were 52 not $A$ not $X$-sequences. The stimuli were presented for $150 \mathrm{~ms}$ with a fixed inter stimulus interval of $1500 \mathrm{~ms}$. The complete task took about 15 minutes.

\section{EEG recording}

The 30 active EEG electrodes were mounted in an electro-cap (Electro-Cap, inc., USA) and connected to a 40 channel NuAmps DC portable amplifier (Neuroscan, inc., USA). The standard electrode locations used were $\mathrm{Fz}, \mathrm{Cz}, \mathrm{Pz}_{Z}, \mathrm{Oz}, \mathrm{F} 7, \mathrm{~F} 8, \mathrm{~F} 3$, F4, C3, C4, T7, T8, P3, P4, P7, P8, O1, O2, Fp1, Fp2, FT7, FT8, FC3, FC4, TP7, TP8, CP3, CP4, FCz and CPz. The ground electrode was placed $2 \mathrm{~cm}$ anterior of the $\mathrm{Fz}$ electrode and the reference electrode was placed at the left mastoid (A.1). The vertical electro-oculogram (EOG) was recorded from one electrode above and one below the left eye and the horizontal EOG from the left and right outer canthi of the eyes. Electrode impedance was measured with a portable impedance meter (F-EZM4A, Grass Inc. USA) and kept below $5 \mathrm{kOhms}$ for the EEG and below $8 \mathrm{kOhm}$ for the EOG. EEG acquisition was controlled by Neuroscan 4.2 software with a band-pass filter of $0.05-30 \mathrm{~Hz}$ and a continuous sampling rate of $250 \mathrm{~Hz}$. Stimulus presentation and acquisition of the behavioural data was controlled by ERTS software (Version 3.18, BeriSoft Cooperation, Germany).

\section{Procedure}

Upon arrival the procedure of the study was explained to the participant. The participants were told that they were asked to participate in three "computer games" and that electrodes would record what the brain was doing while they were playing the computer games. During electrode application one of the researchers asked the questions from the Edinburgh Handedness Inventory. During EEG-recording the participant was seated in a quiet room, $60 \mathrm{~cm}$ in front of a VGA monitor. The two researchers were seated in the same room, behind or 
beside the participant. Before starting the task the following instruction was given: "In a moment pou will see different single letters, which will appear in the middle of the screen. For example an $A, B, C$ or $X$. You have to press the button as soon as possible when you see. the letter X. But only when the letter $X$ is preceded by the letter $A$. First yon get two practice sessions to get used to the stimuli presentation and button press.

There were three short pauses between the test blocks of the CPT-AX task to enable the participant to readjust and chat with the researchers. The subjects also performed a visual and auditory oddball task. Upon completion of the computer tasks the electro-cap was taken off and the subject was asked to fill in Raven's Standard Progressive Matrices. The entire experiment lasted about two and a half hours.

Analyses

The ERP data were extracted by Neuroscan 4.2. Baseline correction was performed using a $100 \mathrm{~ms}$ pre-stimulus interval of a $1000 \mathrm{~ms}$ epoch. Only trials. with correct responses were included and individual EEG sweeps were rejected when the amplitude exceeded $1.75 \mathrm{iV}$. All rejected and accepted sweeps were visually inspected. Peak latency and amplitude for the $N 200$ was picked within a 160-300 ms window after stimulus onset and for the P300 within a $230-580 \mathrm{~ms}$ window. Both the behavioural and ERP measures were subjected to a Generall Linear Models- repeated measures ANOVA using SPSS (version 11.0). Subjects with a value of two standard deviations or more from the group mean were excluded from further analyses. In case of significant interactions post-hoc tests were performed using one-way ANOVAs. All two-tailed significance levels were kept at $p<0.05$ and in case of a violation of the sphericity assumption a Huynh-Feldt correction was applied.

\section{RESULTS}

Behavioural measures

For the Raven's Standard Progressive Matrices (RSPM) non-verbal IQ test the control group scored higher than the disomy and deletion PWS groups with respective raw test scores of $57(\mathrm{~s} . \mathrm{d} .=2.5), 21(\mathrm{~s} . \mathrm{d} .=5.9)$ and $24(\mathrm{~s} . \mathrm{d} .=5.9)$. The disomy group scored within the $5^{\text {th }}$ percentile and those of the deletion group within the $10^{\text {th }}$ percentile of scores based on a reference group of military and civilian subjects in the age from 20 to 65 years from the United Kingdom (Raven ef al. 1983). The control group scored within the $95^{\text {th }}$ percentile. 
For hits or comrect responses to the target (A-X) stimulus in the CPT-AX task only the difference between the control and disomy group was significant $(F(1,20)=$ $11.03, \mathrm{p}<0.003)$ with $98 \%(\mathrm{~s} d .=3.7)$ and $75 \%(\mathrm{~s} d=22.4)$ hits respectively. The deletion group had $87 \%$ (s.d. $=13.3$ ) correct hits. The percentage false alarms, or pressing a button to anything other than a $\mathrm{A}-\mathrm{X}$ combination, showed a similar group trend with a significant difference $(F(1,20)=5.13, p<0.035)$ between controls and the disomy group, of respectively $0.1 \%(\mathrm{~s} . \mathrm{d} .=0.2)$ and 5.9 $\%($ s.d. $=8.5)$. The deletion group had $1.6 \%(5 . \mathrm{d}$. $=2.2)$ false alams and did not significantly differ from controls. Reaction times differed between the control and disomy group (362 ms, s.d. $=65.8$ vs $541 \mathrm{~ms}$, s.d. $=170.5, \mathrm{~F}(1,20)=10.58, \mathrm{p}<$ 0.004 ) and between the disomy and the deletion group (RT deletion group $=398$ ms, s.d. $=84.3, \mathrm{~F}(1,20)=6.21, \mathrm{p}<0.022)$.

Taken together these behawioural results suggest that the disomy PWS group has a lower $\mathbb{R}$ aven $[Q$, reacts slower, has fewer hits and committed more false alams for the CPT-AX task than the deletion and control group.

The event-related brain potentials

The inhibition related ERP components of interest in the present task are the N200 and P300 that are mainly elicited in the AnotX-condition (Nogo-condition) and to a lesser extend in the AX-condition (Go condition). The grand average ERP waves for the three groups of the Go-versus Nogo condition at mid-line electrodes $\mathrm{Fz}, \mathrm{Cz}_{*} \mathrm{Pz}$ and $\mathrm{Oz}$ are displayed in figure 1. It can be observed in this figure that the $\mathrm{N} 200$ component at electrode $\mathrm{Fz}$ (negative going amplitude peak around $200 \mathrm{~ms}$ after stimulus onset) is more negative for the Go condition than for the Nogo condition difference and that this difference is most pronounced in the control group. There is also a P300 (positive peak between $300-500 \mathrm{~ms}$ ) at the ellactrodes Fz and Cz that is larger in the Nogo than in the Go condition in control and deletion group. For the disomy group (middle column) this component is flattened. At visual inspection the ERPS of the disomy group clearly stand apart from the deletion and control group.

\section{The $N 200$}

For the N200 peak latency after stimulus onset there was an overall significant difference across the electrodes $(\mathrm{F}(2.13 / 23)=16.74, \mathrm{p}=0.000)$ with decreasing latencies from the anterior to posterior electrodes, but no difference between the Go and Nogo task condition and groups.

The peak amplitude of the $N 200$ also differed between electrodes $(F(3,22)=$ $34.374, \mathrm{p}<0.000$ ) and there was an interaction effect between the Go-Nogo task condition and electrodes $(\mathrm{F}(3,22)=3.079, \mathrm{p}<0.05)$ with smaller amplitudes for 

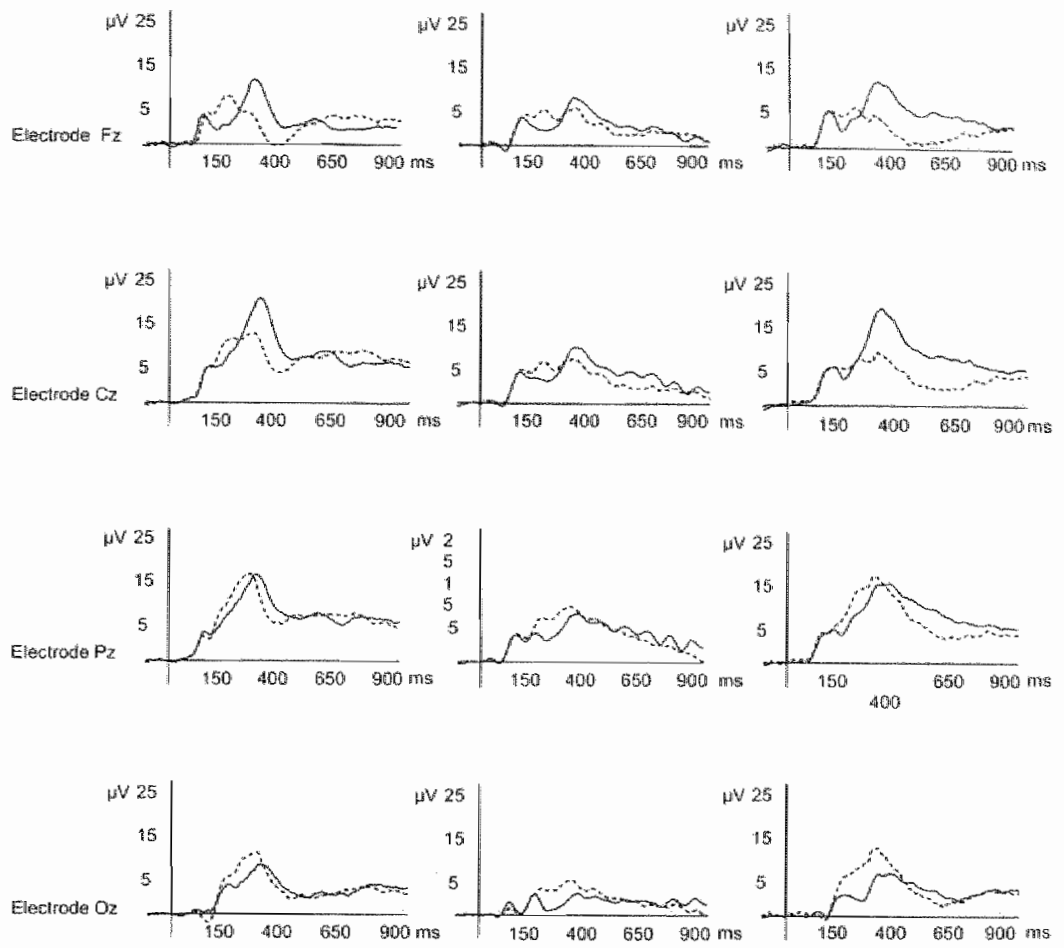

\section{Go Condition $(A X)$}

NoGo Ciondition (AnotX)

Figure 1. Grand average $(n=10)$ ERPS recorded from the $F_{Z}, C Z, P z$ and $0 z$ electrodes to the Go condition (solid line) and NoGo condition (dotted line) in a continuous performance task (CPT-AX). The $y$-axis in each graph indicates the amplitude in $\mathrm{V}$ and the $\mathrm{x}$-axis the time in ms after stimulus onset. The left column depicts the ERPS of the control group, the middle column of the maternal uniparental disomy PWS group and the right column of the parental deletion PWS group. The arrows indicate the 'N200" and 'P300' ERP components that are included in the analyses

the Nogo than the Go condition at Fzand $\mathrm{Oz}$ electrode locations. The latter indicates a successful Go-Nogo task modulation for the N200. A highly significant 3-way interaction with groups (Condition* Electrode* Group, $F(6,46)=3.775$, $p<0.007)$ resulted from a significant task condition effect at the electrodes $F z$ and Oz in the control group only $(t(9)=4.462, \mathrm{p}<0.007)$. Thus despite the fact that 
N200 task condition effects can be observed for the disomy and deletion PWS groups in Figure 1 , these differences were not statistically significant.

\section{The P300}

The peak latency of the P 300 was shorter to the $A X$ condition than to the Arot $X$ $(F(1,18)=9,245, p<0.007)$. A main effect for group was caused by a longer P300 peak latency for the disomy as compared to the control group (390 versus $350 \mathrm{~ms}$, respectively). The averaged P300 latency of the deletion group did not significantly differ from the controls.

P300 amplitude showed a similar trend than the P300 latencies with a main group effect $(\mathrm{F}(2,25)=5.726, \mathrm{p}<0.009)$ due to overall increased amplitudes in the control vs. disomy group $(18.1 \mathrm{iV}$ vs. $11.8 \mathrm{iV}, \mathbb{F}(1,17)=8.83, \mathrm{p}<0.009)$ and for the delations versus disomy group $(1.9 .9$ iV vs. $11.8 \mathrm{iV}, F(1,16)=12.58, \mathrm{p}<$ $0.003)$. Again there was no significant difference between the delletion and control group.

\section{DISCUSSION}

The present study addresses behaviour and event-related brain potentials to a CPT-AX task of people with PWS caused by paternal deletion, by uniparental. maternal disomy and of age matched normal controls. The scores on the Standard Progressive Matrices (SPM) were lower for the disomy than the deletion PWS group (5 $5^{\text {th }}$ vs. $10^{\text {th }}$ percentile) and the PWS groups scored lower than the controls. (95 $5^{\text {th }}$ percentile). The behavioural data of the CPT-AX task showed that the disomy group performed worse than controls and deletions in that they had slower reaction times, fewer hits and more false alams. Both the deletion and disomy PWS groups failed to show the typical task modulation for the N200 ERP Component, suggesting impared brain activation for modality specific early inhibition processes. For the P300 ERP component only the disomy group differed from both controls and the deletion group, suggesting that late general inhibition. processes arc only impaired in persons with uniparental matermal disomy.

On the Raven's Standard Progressive Matrices (RSPM) the controls performed above the $9^{\text {th }}$ percentile. The deletion group performed below the $10^{\text {th }}$ percentile and should be classifed as "definitely below average in intellectual capacity". The disomy group performed at the $5^{\text {th }}$ percentile and are classified as intellectual impared'. The RSPM measures a person's ability to fom perceptual relations and is a measure of general non-verbal intelligence. Roof et al. (2000) found that people with disomy had a higher average verbal intelligence (VIQ) in comparison to those with deletions, whereas there was no significant difference in general 
performance, or non-verbal intelligence (PIQ). Whittington er al. (2004) found that although their disomy PWS group was verbally superior to the delletion PWS subgroup, the deletion group had better non-verbal/perfomance capacities than the disomy patients. This finding is confirmed in the present study.

Lower performance and slower reaction times on the CPT-AX task sets the disomy group apart from the deletion and control group. However, lower performance and slower responses to a CPT-AX task is not specific to this group of patients. Strandburg et al. (1996) found an increased number of percentage false allarms and decreased percentage of hits, together with a prolonged reaction time on the CPT-AX task for ADHD and Schizophrenia groups. It may be more surprising that the deletion group performs so close to the controls, as they have a evidently lower non-verbal IQ as measured with the RSPM and have been reported to have inhibition related problems such as obsessive compulsive behaviour that are typical for PWS. The deletion group may have developed some compensatory strategies that help them to achieve this level of performance on the CPT-AX. The suggestion of compensatory strategies in the deletion groups also is supported by the ERP data.

The peak latency analyses of the $\mathrm{N} 200$ and P300 showed a significant delay for the $\mathrm{P} 300$ for the disomy group as compared to controls. The delayed reaction times to the CPT-AX task in the disomy group therefore appear to be mainly related to delayed activations of late general inhibition processes (P300), while the modality specific early inhibition processes (N200) are not significantly delayed. The N200 and P300 peak latencies in the deletion group are not delayed and their reaction times do not differ from the control group.

Typically, the $\mathrm{N} 200$ amplitude modulation is most pronounced at electrode $\mathrm{F}_{\mathrm{Z}}$ with a more pronounced N200 for the Nogo as compared to the Go condition (Jonkman et al. 2003). This is exactly what is found for the control group. Although the grand average ERP waves (Figure 1) suggest a similar, albeit smaller, N200 effect in the deletion and disony PWS group, this Go-Nogo N200 condition effect failed to reach significance. It is for the P300 amplitude that the disomy group differs most clearly from the control and deletion group. The control and deletion group show a clear P300 with larger amplitudes to the Nogo- as compared to the Go condition. The disomy group on the other hand shows a very small P300 with minor differences between the Go- and Nogo condition. Post-hoc analyses of the Go-Nogo condition effect within the disomy group at the electrodes $\mathrm{Fz}$ and $\mathrm{Cz}$ revealed that this condition effect was not significant. The fact that brain activity is not modulated in the disomy group indicates an impaired late general response inhibition process. These increased inhibition related problems in the disomy group are consistent with observations by Boer ef al. (2002) that disomy patients have a much higher risk of developing affective disorders with psychotic features as they grow older. Also, Kiehl et al. (2000) reported in an ERP 
study using a visual Go-Nogo task that schizophrentc patients and psychopaths showed smaller and absent $\mathbf{N} 200$ effects and a reduced P300 effect. These ERP finding resemble the present $\mathbb{E R P}$ effects for the disomy group.

There is evidence that the $\mathrm{P} 300$ may be relevant in relation to OCD symptoms. Russo et al (2000) reported that $O C D$ patients produced the same large activation for the P300 in the target and non-target Go-Nogo condition. They interpreted this as a misallocation of cognitive resources leading to a $\mathrm{P} 300$ over activation. Russo et al. (2000) found that there was no significant difference between OCD patients and controls for the $N 200$. As in the present disomy group the OCD patients faled to show the typical P300 condition effect, but unlike the OCD patients the disomy patients in this study also failed to show a N200 condition effect, and their P300 is significantly smaller instead of larger. The absence of a P300 condition effect and smaller $P 300$ amplitudes have been also been reported in children. Jonkman ef al. (2003) reported that 9 to 11 years old normal children do not display the fronto-central Nogo P300 wave that is usually observed in adults. They related this finding to an underdeveloped response inhibition system. Interestingly the children did show an adult $N 200$ effect, while they had more false alarms and higher impulsivity scores as compared to the adults. The developmental findings in the Jonkman et al. (2003) study thus suggest that the integrity of the $\mathrm{P} 300$ is more related to the behavioural data than the N200. The present data support this hypothesis in that both PWS groups have non-significant $\mathrm{N} 200$ effects, and it is only the disomy group that exhibits worse performance at the behavioural level and a clearly anomalous Nogo P300.

The psychomphysiological data in this study support previous psychological findings of differences in phenotype between parental deletion and matemal. uniparental disomy PWS subgroups. Although both PWS subgroups revealed atypical carly modality-specific inhibition (N200) processes, only the disomy group revealed impaiments in late response indubition processes (P300 + behaviour). These qualitative differences give further support to recent articles indicating that these PWS subgroups should be considered as a nosological different group of patients who ma beneft from a different approach and distinct treatment programs. The present sudy is the first to present experimental physiological support to earlier reported psychological differences between deletion and disomy PWS subgroups.

\section{References}

Beardsmore A., Doman T., Cooper S.A. \& Webb T. (1998) Affective Psychosis and Prader-Willi Syndrome Jounal of Intellotnal Disability Research 42, 463-71. 
Boer H., Holland A., Whittington]., Butlery., Webb T. \& Clarke D. (2002) Psychotic illness in people with Prader-Willi syndrome due to chomosome 15 maternal unparental disony. Lancet 359, 135-36.

Burd L., Vesely B., Martsolf J. \& Korbeshian J. (1990) Prevalence smdy of Prader-Will syndrome in North Dakota. American Jownal of Mediat Genetics 37, 97-99.

Cassidy S.B. Forsythe M., HeegerS., Nicholls R.D., Schork N, Benn P. \& Schwartz S. (1997) Comparison of phenotype between patients with Pader-Willi syndrome due to delerion 15q and uniparental disomy 15. American Joumal of Medical Genetics 68, 433-40.

Cassidy S.B., (1984) Prader- Willi syndrome. Curvent Problents in Pediatric 14, 1-55.

Cassidy S.B. \& Schwartz S. (1998) Prader-Willi and Angelman syndrome. Disonders of genomic imprinting. Medicine (Baltimore) 77, 140-.51.

Clarke D.J., Boer H., Chung M.C., Sturmey P. \& Webb T. (1996) Maladaptive behaviour in Prader-Willi syndrome in adult life Joumal of Intellectual Disability Researh 40, 1.59-65.

Clarke D.J., Boer H., Whittington J., Holland A., Butler J. \& Webb T. (2002) Prader-Willi syndrome compulsive and ritualistic behaviours: The first population-based survey. The British Joumal of Psydiatry; The Jownal of Mental Science 180, 358-62.

Curfs L.M.G. \& Fryns J.P. (1992) Prader-Willi syndrome: A review with special attention to the cognitive and behavioural profile. Bith Deferts Original Anticte Series 28, 99-104.

Dykens. E.M., Cassidy S.B. \& King B.H. (1999) Maladaptive behaviour differences in Prader-Willi syndrome due to paternal deletion versus matemal uniparental disony. Anerican Joumal of Mental Retardation 104, 67-77.

Dykens E.M., Hodapp R. M., Walsh K. \& Nash L. (1992) Profiles, comelates and trajectories of intelligence in individuals with Prader-Willi syndrome. Joumal of the American Acadenty of Child and Adolescent Psychiatry 31, 913-27.

Dykens E.M. \& Kasari C. (1997) Maladaptive behaviour in children with Prader-Willi syndrome, Down syndrome, and non-specific mental recardation. Antrican foumal of Mental Retardation 102, 228-37.

Dykens E.M. Leckman J.F. \& Cassidy S.B. (1996) Obsessions and compulsions in Prader-Wrilli syndrome. Joumal of Child Psydology and Psychatry, and Alled Disciplines. 37 , 995-1002.

Fabiani M. Gratton G. \& Coles M.G.H. (2000) Event-related brain potentials: Methods, theory and applications. In J. Cacioppo L. Tassinary G. Berntson (Eds), Handbook of Psychophysiology. New York, NY: Cambridge University Press 53-84.

Falkenstein M., Hoomann J. \& Hohnsbein J. (1999) ERP components in Go/Nogo casks and their relation to inhibition. Acta Psphologica 101, 267-91.

Holland A.J., Whittingtony E., ButlerJ. V., Webb T., Boer H, \& Clarke D. (2003) Behavioural phenotype associated with specific genetic disorders: Evidence from a population-based study of people with Prader-Willi syndrome. Psychological Medicine 33, 141-53.

Holn V.A., Cassidy S.B., Buter M.G., Hanchete J.M., Greenswag L.R., Whitman B. Y. \& Greenberg F. (1993) Prader-Willi syndrome. Consensus diagnostic criteria. Pandiatrits 91 , $398-402$. 
Jonkman L.M., Lansbergen M. \& Stauder J E.A. (2003) Developmental differences in behavioral and event-related brain responses associated with response preparation and inhibition in a go/nogo task. Pyohophysiology 40, 752-61.

Kiehl K.A., Smith A.M. ,Hare R.D. \& Liddle P.F. (2000) An event-related potential investigation of response inhibition in schizophrenia and psychopathy. Biological Psychiatry 48 , 210-221.

Oldfeld R.C. (1971) The assessment and analysis of handedness: the Edinburgh inventory. Neuropsycholugia $9,97 \mathrm{~m} 113$.

Overtoom C.C.E., Verbaten M.N., Kemner C., Kenemans J.L., Van Engeland H. \& Buitelar J.K. (1998) Associations between event-related potentials and measures of attention and inhibition in the continuous performance task in children with ADHD and nomal controls. Jowmat of the Ameriam Aademy of Child and Adolestem Psydiatry 37, 977-85.

Pliszka S.R., Liotti M. \& Woldorff M.G. (2000) Inhibitory Control in Children with Attention-Defict/Hypenactivity Disorder. Event-Related Potentials identify the Processing Component and Timing of an Impared Right-Frontall Response-Inhibition Mechanism. Biological Psyliatry $48,238-46$.

Raven, Rawen J.C., Court J.H. \& Raven J. (1983). Manual for Raven's Progressive Matrices and Vocabulary Scales (Section 3). Standard Progressive Matrices (1983 edition). London: Lewis.

RoofE., Stone W., Maclean W., Feurer L.D., Thompson T. \& Butler M.G. (2000) Intellectual characteristics of Prader- Willi syndrome: Comparison of genetic subtypes. Journal of Intellectual Disability Research 44, 25-30.

Stauder J.E.A., Brinkman M.J.R. \& Curfs L.M.G. (2002) Multi-modal P300 deflation of event-related brain activity in Prader-Willi Syndrome. Neuroscience Letter 327,99-102.

Strandburg R.J., Marsh J.T., Brown W.S., Asamow R.F., Higa J., Harper R. \& Guthrie D. (1996) Continous- Processing- Related Event-Related Potentials in children with Attention Deficit Hypenactivity Disonder Biological Psychiary 40, $964-980$.

Symons F.J., Buter M.G., Sanders M.D., Feturer I.D. \& Thompson T. (1999) Self-Injurious behavion and Prader-Willi syndrone: Behavioral forms and body locations. American Jourual of Montal Retardation 104, 260-69.

Verhoeven W.M.A. Tuinier S. \& Curfs L.M.G. (2003) Prader-Willi syndrome: the psychopathological phenotype in uniparental disomy. Joumat of Medical Genetics 40: 112.

Vogels A. Mattijs G., Legrus E., Devriendt K \& Fryns J.P. (2003) Chromosone 15 matemal uniparental disomy and psychosis in Prader-Willi syndrome. Jownal of Medical Genetics 40 . $72-73$.

Whittington J.E., Holland A.J., Webb T., ButlerJ.V., Charke D.J. \& Boer, H. (2004) Cognitive abilities and genotype in a population based sample of people with Prader-Willi syndrome. Joumal of Intellectual Disability Research 48, 172-87.

WhitingtonJ.E., Holland A.J. Webb T., ButlerJ.V., Clarke D.J. \& Boer H. (2002) Relationship between clinical and genetic diagnosis of Prader-Will syndrome. Jourwal of Medical Gentio 39, 926-32. 
Whitrington J.E., Holland A.J., Webb T., Butler J. V., Clarke D.J. \& Boer H. (2001) Population prevalence and estimated birth incidence and mortality rate for people with Prader-Willi syndrome in one UK health region. Joumal of Medieal Cenetis 38, 792-98.

Wigren M. \& Hansen S. (2003) Rituals and compulsivity in Prader-Willi syndrome: profile and stability. Joumal of Intellectnal Disability Research. 47, 428-38. 

Chapter $7 b$

\section{Prader-Willi syndrome:}

\section{Behavioural differences between deletion and uniparental disomy subtypes}

Submitted as:

Boer H, Stauder J.E.A., Gerits R.H.A., Tummers A., Whittington J. \& Webb T., Curfs L.M.G. Prader-Willi syndrome: Behavioural differences between deletion and uniparental disomy subtypes. 


\section{Abstract}

Badkgromd Prader-Willi syndrome (PWS) is a rare genetic disorder which in approximately $70 \%$ of cases is caused by a deletion at the locus $15 q 11-13$, involving claromosome 15 of paternal origin and in about $25 \%$ by maternal chrom mosome 15 uniparental disomy (UPD). Recent sudies have found differences in the behavioural phenotype between these PWS genetic subtypes. The present study compares experinental behaviounal findings in a group of patients with deletion, a group with maternal uniparental disomy PWS and a control group. Method Behaviour was monitored in response to an auditory and a visual oddball task in adults with paternal deletion PWS $(\mathrm{n}=11)$, maternal uniparental disomy PWS $(n=11)$ and nomal controls $(n=11)$. The dependent behavioural variables of the casks were reaction time, number of hits and number of misses. We also used the Baron-Cohen "Reading the Mind in the Eyes" test in these three groups. Results In the auditory task the deletion group had more misses than the contro] group. In the visual task the UPD group had more misses and longer reaction times than the control group. Both the deletion and the UPD subgroups scored significantly lower on the Reading the Mind in the Eyes test than the control group.

Condusions Our results indicate that those with deletion have relatively better visual skills, and those with UPD relatively better auditory skills. We also found some support for the previous finding of high scores on items relating to autistic spectrum disorder in PWS.

\section{Introduction}

Prader-Willi Syndrome (PWS) is a genetically detemined developmental disorder. The syndrome was first described by Prader et al. (1.956) and results in severe hypotonia at birth and failure to thrive in the early years. From the age of about wo years individuals with PWS develop an almost insatiable appetite that can tesulc in extrene obesity unless the food environment is controlled (Holland et a. 1993). Most people with PWS have a mild or borderlne mental retardation. Specific behavioumal problems, including sleep disturbance, temper tantrums, lability of mood, and repetitive speech are common (Clarke et al. 1996). PWS is caused by the absence of maternally imprinted genes at the chromosomal locus 15q11-13 (Gallagher ef at. 2002). About 70\% of people with PWS have a deletion affecting chromosome 15 (q11-13) of paternal origin (Ledbetter ef al. 1981). The majority of the others have a chromosome 15 matemal disomy (Nicholls et al. 1989), with a small number having other chromosomal abnomalities, such as imprimting centre mutations (Robinson of al. 1991; Buiting et al. 1995). The observation that either a similar deletion on the chromosome 15 of maternal 
origin, or the presence of a patemal chromosome 15 disomy gives rise to the phenotypically different Angelman syndrome, led to the conclusion that PWS was. due to the loss of expression of specific paternally imprinted gene(s) located within the PWS critical region of chromosome 15 (Kuwano ef al. 1992). The incidence of PWS has been estimated to be about 1:20000 live births (Whittington et al. 2001). The study of people with PWS with UPD and deletion can provide information about the impact of genes on clinical features. Although the main manifestations of both PWS due to deletion and PWS due to UPD are similar, there are a number of differences, for instance hypopigmentation being more common in the deletion group (Gillessen-Kaesbach et al. 1995; Mitchell et al. 1996). A recent finding is that those with UPD are much more likely to become psychotic in adult life (Boer et af. 2002). Cassidy et al. (1997), in a retrospective study, found no differences in overall IQ between individuals with PWS due to deletion and those with UPD, but they found that unusual skills with jigsaw puzzles and skin picking were less common in the UPD group. Roof et al (2000) compared intellectual characteristics of genetic subtypes of PWS. They concluded that individuals with UPD had a significantly higher average verbal IQ than those with a deletion. Roof et al concluded that those with a deletion had a performance $I Q$ that was on average four points higher than their verbal IQ and that the verbal IQ of individuals with UPD was on average eight points higher than their performance IQ. Whittington et al. (2004), in a population study, looked at people with PWS due to deletion and due to UPD, with a comparison group of people with learning disability, and found that the group with UPD had a cognitive strength in vocabulary and a weakness in coding (speed) compared with the other two groups.

Stauder et al. (2002) compared visual and auditory oddball tasks of ten adults with PWS with ten normal controls in a cognitive psycho-physiological study in which event-related brain activity was recorded. They found that in people with PWS the auditory modality was more affected than the visual modality. However, they did not distinguish between those with deletion and UPD.

Stauder et al. (submitted) compared event-related brain potentials during a CPT-AX task (a task which can detect inhibition-related problems; subjects are asked to press a button when the letter $X$ appears, but only if this has been preceded by the letter A) in people with PWS due to deletion with individuals with UPD and with age matched nomal controls. The UPD group had lower performance and slower reaction times than the deletion and control groups. The P300 amplitude was lower in the UPD group than in the deletion and control groups, which implies more inhibition related problems. This is consistent with the finding by Boer et al. (2002) that individuals with UPD have a high risk of affective psychoses in adult life.

Velman et al. (2004) reported that people with PWS caused by deletion and by UPD had relatively high scores on the Autism Screening Questionnaire but that 
compared to people with deletion, those with UPD had more autistic symptoms. They found that people with PWS due to UPD had difficulty with one aspect of visual perception, i.e. integrating parts of a perceptual array into a unified construct. Velman ef al. proposed that it is possible that, as they had found that those with UPD had more symptoms suggestive of autism, one explanation for this would be that such a bias towards local rather than global aspects of visual processing is also found in autistic spectrum disorders. This would also be a possible explanation for the finding of better visual memory found in those with UPD compared with deletion, as specific skills in visual memory (attention for details) is also found in some people with autistic traits.

In the present study we aimed to examine the differences between the deletion and UPD subgroups of PWS using behavioural information from neuro-physiological studies in PWS, and we used the Baron-Cohen Adult Eye test to compare autistic characteristics between the two main PWS genetic subgroups.

\section{Materilials and Methods}

Twenty-two participants with PWS (11 with deletion and 11 with UPD) and 11 nomal controls took part in this study. Most PWS patients were recorded in England (eight with deletion and seven with UPD) and the remaining seven individuals with PWS, and the controls, were recorded in the Netherlands. There were seven men and 4 women in the deletion group and four men and seven women in the UPD group. The control group consisted of six males and 5 females. The average age for these groups was 26.7 years ( $\mathrm{sd}=7.35$ ) for the deletion group, 27.7 years $(\mathrm{sd}=8.81)$ for the UPD group and 27.3 years $(\mathrm{sd}=8.25)$ for the control group. These ages do not differ significantly between groups. Two of the subjects with UPD were treated with anti-psychotic medication for a psychotic disorder, and one was prescribed antidepressant medication, but none were suffering from psychotic symptoms at the vime of the assessment.

The subjects were asked to participate in two tasks, a wisual and an auditory oddball task. During the visual oddball task subjects were asked to sit $50 \mathrm{~cm}$ in front of 17" computer monitor and to look at faces appearing on the screen looking right, left and straight ahead ("at you"). Subjects were asked to press the button as quickly as possible if the faces were looking at them. During the auditory oddball task the subjects were asked to respond to three pure sinus tones at $70 \mathrm{~dB}$ with pitches of 880,440 and $220 \mathrm{~Hz}$. Participants were asked to press the button when they heard the $880 \mathrm{~Hz}$ tone. There were short pauses between the blocks of each task and breaks between the tasks. The entire experiment lasted about two and a half hours. All participants were asked to complete the "Reading the Mind in the Eyes" test (Baron-Cohen, 2001). This test consists of photographs of the eye region of 36 
different male and female faces, taken from magazines. The faces were standardised to 15 by $10 \mathrm{~cm}$. The participants are asked to choose one of four mental states that are printed on the comers of each page, and to judge the gender of the person in the picture. The test aims to measure the ability of the participants to put themselves into the mind of someone else (Theory of Mind).

Statistical analysis was performed using SPSS, using Bonferroni correction to correct for multiple tests.

\section{Results}

The results of the Reading the Mind in the Eyes test are shown in Table I, including the mean score on number of emotions scored correctly and the mean score on number of hits of determining the gender correctly, with standard deviations for each. Statistical analysis shows a significant group effect for the emotion score $(F(2,27)=42.16, p<0.000)$, but not for gender. There are significant differences both between the control and deletion groups $(\mathbb{F}(1,19)=64,18, p<0.000)$ and between the control and UPD groups $(F(1,18)=49,00, \mathrm{p}<0.000)$.

During the auditory task the percentage of hits and false alarms ('misses', or pressing the button incorrectly) were measured, as well as the reaction times. The mean percentages and reaction times are displayed in table 2 . A significant group effect was found for the percentage of hits $(F(2,30)=3.462, p<0.044)$ and for the percentage of false alarms $(F(2,30)=5.707, p<0.008)$, but not for reaction time. Table 3 shows the statistical analyses of the differences between mean percentages of hits and between the mean percentage of false alarms in the auditory oddball task using the post hoc test. There is a significant difference between the control and UPD groups in the percentage of hits, and between the deletion and controls in the percentage of false alarms.

In the visual oddball test a significant group effect was found for the percentage of hits $(F(2,30)=4.558, p<0.019)$, the percentage of false alarms $(F(2,30)=8.015$, $p<0.002)$, and there was a significant group effect for the reaction times $(F(2,30)$ $=5.549, \mathrm{p}<0.009$ ). Table 4 shows the mean percentages and standard deviations of hits and false alarms and the reaction time and standard deviations during the visual oddball tasks.

Table 5 shows the statistical analyses of the differences in the mean percentages of hits and of false alarms as well as the statistical differences in the reaction times in the visual oddball task between the deletion, UPD, and control groups using the post hoc test. There is a significant difference between the control and UPD groups in the percentage of hits, and between the deletion and controls as well as between UPD and control groups in the percentage of false alarms. Reaction times are significantly lower in the UPD group compared to the control group. 
Table 1. Reading the Mind in the Eyes Test (Baron-Cohen, 2001). Mean number and standard deviations of correctly scored items on emotion and gender

\begin{tabular}{lllll} 
& Emotions & SD & Gender & SD \\
\hline Deletion & 12 & 3.3 & 34 & 0.6 \\
UPD & 13 & 3.3 & 33 & 3.1 \\
Control & 25 & 4.1 & 34 & 1.1 \\
\hline
\end{tabular}

Table 2. The mean percentages and standard deviations of hits and false alarms and the reaction times and standard deviations on the auditory oddball tasks.

\begin{tabular}{lllllll} 
& Hits & \multicolumn{3}{c}{ False alarms } & \multicolumn{2}{c}{ RT to Hits } \\
& $(\%)$ & SD & $(\%)$ & SD & (ms) & SD \\
\hline Deletion & 85 & 22.67 & 9.8 & 11.36 & 484 & 122.4 \\
UPD & 78 & 25.31 & 3.0 & 3.02 & 579 & 225.96 \\
Control & 99 & 1.34 & 0.3 & 0.88 & 465 & 114.4 \\
\hline
\end{tabular}

Table 3. Statistical analyses of the differences between mean percentages of hits and the mean percentages of false alarms in the auditory oddball task using the post hoc test.

\begin{tabular}{llll}
\hline Measure & Deletion vs. UPD & Deletion vs. Control & UPD ws. Control \\
Hits & $p=1.000$ & $p=0.259$ & $p=0.046$ \\
False Alarms & $p=0.076$ & $p=0.008$ & $p=1.000$ \\
\hline
\end{tabular}

Table 4. The mean percentages and standard deviations of hits and false alarms and the reaction time (RT) and standard deviations during the visual oddball tasks

\begin{tabular}{lllllll} 
& Hits & \multicolumn{3}{c}{ False alarms } & RT to Hits \\
& $(\%)$ & SD & $(\%)$ & SD & (ms) & SD \\
\hline Deletion & 82 & 26.2 & 8.50 & 8.67 & 662 & 80.58 \\
UPD & 70 & 31.1 & 12.78 & 9.80 & 708 & 93.06 \\
Control & 100 & 0.0 & 0.11 & 0.24 & 583 & 91.80 \\
\hline
\end{tabular}

Table 5. Statistical analyses of the differences in the mean percentages of hits and the mean percentage of false alarms in the visual oddbal task between the deletion, UPD, and control groups using the post hoc test, as well as the differences in reaction times.

$\begin{array}{llll}\text { Measure } & \text { Deletion vs. UPD } & \text { Deletion ws. Control } & \text { UPD vs. Control } \\ \text { Hits } & p=0.662 & p=0.269 & p=0.016 \\ \text { False Alarms } & p=0.582 & p=0.042 & p=0.008 \\ \text { Reaction Times } & p=0.697 & p=0.140 & p=0.001\end{array}$

\section{Discussion}

In our study both the PWS subgroups scored significantly lower than the control group in the Baron-Cohen Reading the Mind in the Eye test. This gives some support to Velman's (2004) findings that people with PWS have high scores on the Autism Screening Questionnaire, but whereas she reported higher scores on 
the Autism Screening Questionnaire for the UPD group, we found no differences between the deletion and the UPD subgroups. However, our findings need to be taken with some caution, as those in the comparison group did not have a learning disability, and a number of those in the PWS subgroups may have had some difficulties in understanding the terms for the emotions.

Although there were no significant differences in hits, false alarms and reaction times between the deletion and UPD groups, there were clear differences in the behavioural profile of these PWS subgroups in comparison with the control group. In the auditory oddball task the deletion subgroup made more false alarms than the control group. In the visual oddball task the UPD subgroup made more false alarms than the control group, and had longer reaction times. People with PWS caused by deletions have been reported to have better visuospatial skills than those with UPD (Whittington, 2004) and our results appear to give some support to this finding. As it is likely that about 70\% of those with PWS in Stauder et al's (2002) study had a deletion, our results are consistent with their finding that in people with PWS the auditory modality was more affected than the visual modality.

Obsessive-compulsive symptoms have been reported to occur often in PWS (Dykens et al. 1996; Clarke ef al. 2002). These compulsive symptoms may be caused by a deficit in inhibitory control (Pliszka ef al. 2000). This appears to be supported by our finding that both PWS subgroups score relatively poorly in the number false alarms.

A potential weakness of the study is the fact that some of the subjects lived (and tests were performed) in the UK and others in the Netherlands. However, the same researchers were involved in the tasks in both countries and used the same set-up. Our results suggest that there are significant differences between the deletion and UPD subgroups of PWS in how they process auditoryand visual information. The genetic subtypes result in subtly different behavioural phenotypes, and further research needs to investigate the possible causes of these differences.

\section{References}

Baron-Cohen S., Wheelwright S., Hill J, Raste Y. \& Plamb 1. (2001) The "Reading the Mind in the Eyes" Test revised version: A study with normal adults, and adults witl Asperger syndrome or high-functioning antism. Joumd of Child Psydology and Psydratry and Allied Disciplines $42,241-51$.

Boer H., Holland A., Whittington J, ButlerJ., Webb T., \& Clarke, D. (2002) Psychoticillness in people with Prader-Willi syndrome due to chromosome 15 maternal uniparental disony. Lanet 359, 135-136. 
Cassidy S. IB., Forsythe M. Heeger S., Nicholls R. D., Schork N., Benn IP. \& Schwartz S. (1997) Comparison of phenotype between patients with Prader-Willi syndrome due to deletion 15 qand uniparental disomy 15. American Joumal of Medical Genetic 68, 433-40.

Clarke D. J., Boer H. Chung M. C., Sturmey P. \& Webb T. (1996) Maladaptive behaviour in Prader-Willi syndrome in adult life. Jound of Intellectual Disability Reveard 40, 159-65.

Dykens E. M. Cassidy S. B. \& King B. H. (1999) Maladaptive behavior differences in Pader-Willi syndrome due to paternal deletion wersus maternall uniparental disomy. Ameritan Joumal on Mental Retardation 104,67-77.

Gallagher R. C., Pils B., Albalwi M. \& Francke U. (2002) Evidence for the role of PWCR 1/HBII-85 C/D box small nucleolar RNAs in Prader-Willi syndrome. American Jowmal of Human Genetion 71, 669.78.

Holland A. J., Treasure J., Coskeran P. \& Dallow J. (1995) Characteristics of the eating disorder in Prader-Willi syndrome: implications for treatment. Joutnal of Intellectsal Disability Researd 39, 373-81.

Kuwano A., Mutirangura A., Dittrich B., Buiting K., Horsthemke B., Saitoh S., Niikawa N., Ledbetter S. A. Greenberg F., Chinault A. C. \& Ledbetter D. H. (1992) Molecular dissection of the Prader-Willi/Angelman syndrome region (15q11-13) by YAC cloning and FISH analysis. Human Molecular Genetics 1, 417-42.

Mitchell J., Schinzel A., Langlois S., Gillessen-Kaesbach G., Schuffenhauer S." Michaelis R., Abeliovich D., Lerer I., Christian S., Guitart M., McFadden D. E. \& Robinson W. P. (1996) Comparison of phenotype in uniparental disomy and deletion Prader-Willi syndrome: sex specific differences. Anerican Joumal of Medical Genethic 65, 133-6.

Oldfield R. C. (1971) The assessnent and analysis of handedness: the Edinburgh inventory. Neuropsychologia 9, 97-113.

Pliszka S. R., Liotri M. \& WoldorffM. G. (2000) Inhibitory Control in Children with Attention-Deficit/Hyperactivity Disorder: Event-Related Potentials identify the Processing Component and Timing of an Impaired Right-Frontal Response-Inhibition Mechanism. Biological Psychiary, 48, 238 46

Prader A., Labhart A. \& Willi H. (1956) Ein Syndrom von Adipositas, Kleinwuchs, Kryptorchismus und Oligophrenie nach myotoniertigem Zustand im Neugeborenaller.

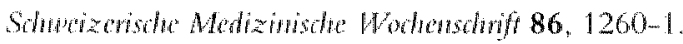

Robinson W. P., Bottam A., Yagung X., Balakrishnan J., Binkert F., Macher M., Prader, A. \& Schinzel, A. (1991) Molecular, cytogenetic and clinical investigations of Prader-Willi syndrome patients. Ancrian Jownal of Haman Generios 49, 1219.34.

Roof, E., Stone, W. Maclean, W. Feurer, I.D., Thompson, T., \& Butler, M. G. (2000). Intellectual characteristics of Prader- Willi syndrome: Comparison of genetic subtypes.

Joumal of Intellectual Disability Researd, 44, 25-30.

Stauder J. E. Brinkman M. J. \& Curf L. M. G. (2002) Multi-modal P3 deflation of event-related brain activity in Prader-Willi syndrome. Nowrosience Lefters 327, 99-102.

Stauder J. E. A., Boer H., Gerits R. H. A., Tummers A., Whitrington 1. \& Curf L. M. G. Differences in behavioural phenorype between parental deletion and matemal uniparental disomy in Prader-Will syndrome. Subrnitted. 
Velman M. W. M., Thompson R. J., Roberts S. E., Thomas N. S., WhitringtonJ. \& Bolton P. F. (2004) Prader-Will syndrome-a study comparing deletion and uniparental disony cases with reference to autism spectrum disorders. Ewopean child E adolssent psydhoty 13 , $42-50$.

Whittington J. E., Holland A. J., Webb T., Butlet J V., Clarke D.J. B Boer H. (2001) Population prevalence and estimated birth incidence and mortality rate for people with Prader Willi Syndrome in one UK Health Region Jomal of Medical Geneno 38, 792-8. 

Chapter 8

Discussion 


\subsection{General Comments}

The studies presented in this thesis give further support to the suggestion that the behaviours associated with PWS constitute at least one classical behavioural phenotype (see e.g. Holland et al. 2003a; Dykens, 2004), with the genetic subtypes (deletion and UPD) resulting in subtly different behavioural phenotypes. The term behavioural phenotype was first proposed by Nyhan in his presidential address to the Society for Pediatric Research in 1971 (Nyhan 1995) in order to stress that the self-injurious behaviour in Lesch-Nyhan syndrome was as much part of the disorder as the plysical findings. O'Brien (2002), in a modified version from that originally suggested by $O^{\prime} B r i e n$ and $Y$ ule (1995) proposed the following definition for 'behavioural phenotype': The behavioural phenotype is a characteristic pattem of motor, cognitive, linguistic and social obsewations that is consistently associated with a biologial disorder. In sowe cases, the behavioual phenotype way constitute a psychatric disorde; in others, behaviows which are not usually regarded as symptoms of psychiatric diagnoses may accur. However, it can now be argued that the studies presented in chapter 6b and 7 (Boer et al. 2002; Stauder et al. submitted; Boer et al. submitted) imply that although the core phenotypes are similar, the two main genetic subtypes of PWS are sufficiently different in presentation that they should be seen as separate behavioural phenotypes (see Holland et al. 2003b). We have attempted to avoid bias, by using comparison groups, and where possible, to use epidemiological studies. To otr knowledge neither comparison studies nor population studies on behaviour in PWS had previously been reported.

\subsection{Population Prevalence}

In some countries genetic records are being coordinated so that all new diagnoses are available in one place. Two recent birth incidence estimations have been made from such records (Smith et al 2003 Vogels et al. 2004). Both studies found a strikingly similat birth incidence to that found in our study (Whittington ef al. 2001; chapter 2). In Australia all infants diagnosed with PWS between 1998 and 2000 were reported), and in all cases the diagnosis was confurmed by genetic testing. Smith at al. (2003) found 30 live births of genetically confimed cases of PWS babies, giving a birth prevalence of 1 per 25000 live births. This is probably a minimum estimate of birth incidence as some cases may have been missed or have gone unreported. Another limitation is that the results of this study are based on three years' data only.

In Fianders a total of 19 children were born during this 8-year period of 1993 to 2000 (Vogels et a 2004). There were a total of just under 507000 births and they estimated a birth incidence of PWS of 1 in 27000 . However, Vogelset al. found a 
much lower prevalence rate of $1: 77000$. Vogels ei al. suggest that one explanation for this difference may be a difference in ascertainment, in that 28 of the PWS patients in our UK (Whittington ef al. 2001) study were given a clinical diagnosis only. However, we estimate that at least $80 \%$ of these 28 patients have a grenetic diagnosis of PWS. Vogels et al. propose that a potential source of error in their results is missing cases. One explanation which would explain the difference in the prevalence data between the UK and the Flanders studies, as well as the fact that the birth prevalence data are very similar, is that Vogels ef al. appear to have described known cases, whereas in the UK study we actively looked for people with PWS.

In view of the remarkable similarities between the three recent population studies on PWS it is likely that the birth incidence of PWS is roughly similar at least in developed countries and that the birth incidence lies between 1:20000 and 1:30 000. The population prevalence is likely to be higher than that estimated by Vogels et al. (2003), probably around 50000 . The mortality rate is more difficult to calculate, but in view of the fact that a number of deaths have been described in chilldren with PWS (Whittington er al. 2002; Schrander-Stumpel et al. 2004), our assumption of a steady death rate of up to $3 \%$ per year may be the best estimate available.

\subsection{Cognitive functioning}

In our population-based study on cognitive abilities in PWS we found support for the findings of Roof et al, that those with UPD had higher verbal intelligence scores than those with deletion and no difference in the average performance (or non-verball) intelligence (Whittington et at. 2004a; chapter 3a), although there were some differences. We found higher verbal abilities in those with chromosome 15 UPD (and a trend towards lower performance abilities), whereas the intellectual abilities of patients with deletions are similar to those of a group of people with general learning disabilities. In addition Roof en al. found that people in the disony group had particular difficulties in coding, and low scores on this subtest. We have suggested that the differences between the genetic subtypes means that there is either an over-or an under-expression of maternally or paternally expressed gene(s) on chromosome 15, and that the two genetic subtypes must have a different pattern of expression of maternally imprinted genes located outside the PWSCR, and of paternally expressed genes located either inside or outside this region.

The percentage of time in schooling spent in special needs schools is a significant predictor of underachievement in the group with PWS, but not in the control (LD) group, possibly as a result of some children with PWS being placed in special needs schools not because of low intelligence, but because of behavioural prob- 
lems, which cannot be dealt with in mainstream schools (Whittington er al. 2004b; chapter 3b). Those in both groups who had achieved as expected in reading, writing and arithmetic had never been to special schools. The group with PWS performed better in reading than in arithmetic, whereas the LD group were equally good at reading and arithmetic. Comparing the deletion group with the disomy group, we found that on average, the disomy group did not underachieve in reading, and that the group with deletion had a poorer achievement on reading, possibly explained by the fact that those with a UPD have a higher score on verbal intelligence than the deletion group

\subsection{Maladaptive behaviour}

Although the studies on behaviour in people with PWS described in appendix 1 and chapter 4 have used comparison groups, a potential weakness in the methodology is that there may have been a selection bias, as we did not use epidemiological samples. However, in our population-based study, we found that the range and nature of behaviours were consistent with findings from other studies (Holland et al. 2003a), including the study described in appendix 1. Eating disorder, temper tantrums, skin picking, mood fluctuations, repetitive questioning and obsessional traits were the most prevalent behavioural characteristics, with relatively low prevalence rates in the comparison group (Holland ef al. 2003a).

Following our study comparing PWS to other deletion syndromes (Clarke and Boer, 1998, chapter 4a), Dykens and Smith (1998) reported a study comparing maladaptive behaviour in children and adolescents with Smith-Magenis syndrome to two matched groups, one with PWS, and one with heterogeneous learning disability. Their results confirmed our findings that "temper tantrums" were among the most problematic behaviours reported by caregivers in both syndromes (although much more so in Smith-Magenis Syndrome). They found that those in the PWS group slept more, and were underactive compared to those in the Smith-Magenis syndrome group, that the PWS group was more prone to social withdrawal, and that skin-picking was more common in PWS. The majority of those in the Smith-Magenis group had self-injurious behaviours, including placing objects in bodily orifices.

The studies presented in this thesis have concentrated mainly on the adult population, although the majority of previous behavioural studies have concentrated on children and adolescents, and few studies have compared the behaviour of adults with PWS with that of children.

Dykens (2004), comparing adults and children with PWS, found that older adults with PWS had significantly reduced levels of maladaptive and compulsive behav- 
iours, and that many adults with PWS over the age of 30 years seemed to mellow. She suggests that one possible explanation for this would be that those with fewer behavioural problems are more likely to survive into older age, al though this may not be the complete explanation (Dykens, 2004). However, we found that aberrant behaviour increased with age in the PWS group, possibly explained by increasing independence from parental controls (Holland et al. 2003a). Living conditions may be one factor, as Greenswag (1987) reported that those who lived alone with little supervision were significantly heavier than others who had more support, and her results imply that people with PWS who lived in specialist provisions for PWS were less likely to be very obese. Further research into the factors which govern survival in people with PWS is clearly needed.

Looking at clustering of behavioural variables in PWS to investigate possible common mechanisms that could account for these variables we found three factors in the factor analysis (Holland et al. 2003; see Table 1). The first factor consisted of the severity of the eating behaviour, lying and stealing. This clustering appears to make sense, as in our experience people with PWS are less likely to lie or steal in relation to issues other than those associated with food. The second factor consisted of obsessive behaviour, temper tantrums, violent/aggressive behaviour and possessiveness. The hypothesis is that such behaviours are related to developmental delay, and that there is a biological basis to this behaviour. For example, parents have reported that aggressive or violent behaviour was almost always reported as occurring only during temper tantrums, so that it is expected that they load on the same factor. The third factor included mood swings, skin picking and argumentativeness. This clustering also has face validity, as parents and carers have often commented that deteriorating behaviour was frequently related to episodes of low mood.

Table 1. PWS and factor analysis, with factor loadings

PWS factor analysis

\begin{tabular}{|c|c|c|c|c|c|}
\hline \multicolumn{2}{|l|}{ Factor1 - eating } & \multicolumn{2}{|l|}{ Factor 2 - personality } & \multicolumn{2}{|c|}{ Factor 3 - emotions } \\
\hline Eating behaviour & 0.82 & Obsessive & 0.58 & Mood swings & 0.50 \\
\hline Lying & 0.88 & Temper tarntrums & 0.73 & Skin picking & 0.68 \\
\hline Stealing & 0.75 & $\begin{array}{l}\text { Possessive } \\
\text { Violent/aggressiwe } \\
\text { Repetitive questions }\end{array}$ & $\begin{array}{l}0.69 \\
0.68 \\
0.49\end{array}$ & $\begin{array}{l}\text { Stubborn } \\
\text { Argumentative }\end{array}$ & $\begin{array}{l}0.75 \\
0.64\end{array}$ \\
\hline
\end{tabular}

From: Holland A.J., Whittington J.E., Butler J., Webb T., Boer H. \& Clarke D (2003). Behawioural phenotypes associated with specific genetic disorders: evidence from a population-based study of people with Prader-Willi syndrome. Psychological Medicine 33, 141-53 
As eating behaviour is a separate factor from other behaviours such as temper tantrums, obsessiveness and mood swings its genetic and neurobiological basis may be different from these other maladaptive behaviours. As eating disorder appears to be always present in PWS, an "all or nothing model" applies (the inturired expression of a single gene has a direct effect on the behavioural characteristic), and it is likely that there is a direct relationship between the abnomal expression of a specific gene in the PWS critical region and the eating behaviour (Holland et al. 2003 a). It is possible that the bypothalamus is involved in the aetiology of eating disorder in PWS. Swab ef al. (1995) found that the number of oxytocin neurones (which inhibit food intake) was significantly reduced in astudy of tissue from the hypothalamus of five people with PWS. Recenty, Goldstone et a. (2002) examined the levels of two other hypothalamic neuropeptides which are important in the regulation of appetite (neuropeptide $\mathrm{Y}$ and agouti-related protein), and found that levels of these proteins were increased in a variety of illnesses, but not in PWS, where they were found at appropriate levels, rather than at the predicted high levels, which had been expected in view of the excessive eating behaviour. Blood levels of the newly discovered orexigenic gut peptide, ghrelin (which has been shown to induce obesity by increasing food intake), have been found to be high in PWS adults compared to obese controls (DelParigi $t$ al. 2002; Goldstone ef al. 2004), and these authors suggest that ghrelin may be in part responsible for the overeating in PWS. Further research is clearly indicated in this area, as this potentially has far-reaching implications, not just for PWS alone.

\subsection{Obsessive-compulsive symptoms}

The obsessive-compulsive behaviours that are seen in PWS are probably best seen as part of a developmental delay, rather than as part of an obsessive-compulsive disorder (chapter 5; Clarke of al. 2002). Holland et al. (2003a) and Wigren and Hansen (2003) reported that the rates of obsessive-compulsive behaviour in people with PWS is present from early childhood, and does not change much with age. The factor analysis described in chapter 8.4 confirms this view, as the item obsessive-compulsive behaviour scored on a difterent factor from skin picking and moodiness. Dykens (2004) reported that in her study, compulsive behaviours did increase from the child to adolescent years, but that compulsive behaviours diminished significantly in older adults with PWS. We have proposed that one action of the PWS genotype is to lead to a specific pattern of atypical and arrested brain development such that the characteristic rituals and compulsions of early childhood continue, and that these behaviours only resolve if the person's development goes beyond this developmental phase (Holland et al. 2003a). 


\subsection{Prader-Willi syndrome and psychotic disorders}

Our population-based study on psychotic illness (Boer et al. 2002, chapter 6b) represents the first epidemiological research of psychiatric disorder in adults with PWS. The strength of this study is its population base that allows an estimate of the prevalence of psychiatric disorders affecting people with PWS to be ascertained free from selection bias. Establishing the exact psychiatric diagnosis can be problematic in people with additional learning disabilities. However, there was a high agreement that cyclical affective disorders with psychotic phenomenology were the main characteristics of the six diagnosed as having a major psychiatric illness. The only person with a chromosome 15 deletion had a less obviously affective disorder. She had previously been reported (in Clarke et al. (1998) as case 2 (G)) and she had been treated with trifluoperazine (anti-psychotic medication) for many years.

For the purposes of comparison we considered published studies of the prevalence rates of psychiatric disorder among people with learning disabilities generally, and those of specific disorders associated with learning disabilities. In a review article Cooper and Collacott (1996) suggested that rates of affective disorder in people with learning disabilities may be as low as $1 \%$ to $5 \%$. Corbett $(1979)$, in his Camberwell study of people with learning disabilities of 15 years and older, found rates of present or past significant affective disorders of $5.5 \%$. A population-based study of adults with Down syndrome reported rates of depression of $11 \%$ (Coillacott et al. 1992). It would therefore seem that the high prevalence rate for severe psychiatric illness in people with PWS is not simply due to having a learning disability, but may represent an increased risk specific to this syndrome.

Strikingly, five of the seven (71\%) of those with severe affective and/or psychotic disorders in our study had uniparental disomy, suggesting that it is this chromosomal type that is particularly associated with the high risk for severe psychiatric disorder. Of the six participants over the age of 28 with a disomy, all six were found to have a major psychiatric disorder. The numbers in this study are small but there is some support from case reports, where psychosis associated with chromosome 15 disomies would also appear to be more common than expected. In Table 2 we have summarised the results of recent articles and case reports. For example, Verhoeven et al. (1998) reported six people with PWS and an affective disorder or cycloid psychosis. Three of the four with known genetics had uniparental disomy. A number of studies have compared the disomy and deletion subgroups in people with PWS. Gillessen-Kaesbach et al. (1995) compared PWS patients with deletion to non-deletion patients and found that those with a deletion were more likely to have hypopigmentation and a lower birth weight than the group without deletion. Cassidy et al. (1997) found that those with disomy had a less typical facial appearance, and were less likely to have minor behavioural characteristics typical 
Table 2. Recent articles describing patients with PWS and major psychiatric disorder

\begin{tabular}{|c|c|c|c|c|c|c|}
\hline \multirow[t]{2}{*}{ Resference } & \multirow{2}{*}{$\begin{array}{l}\text { Numbar of } \\
\text { petients with } \\
\text { affective } \\
\text { psychosis }\end{array}$} & \multirow{2}{*}{$\begin{array}{l}\text { Genetics } \\
\text { known }\end{array}$} & \multirow{2}{*}{$\begin{array}{l}\text { Genetic } \\
\text { abnormaliny } \\
\text { Detetion }\end{array}$} & \multicolumn{2}{|c|}{ Comments } & \\
\hline & & & & Disomy & other & \\
\hline Verhoeven et ol $2003^{2}$ & 23 & 165 & 2 & 14 & 7 & $\begin{array}{l}\text { Includes atl patients in } \\
\text { Verhoeven et al. } 1998\end{array}$ \\
\hline Vagels at al. 2003 & $\sqrt{5}$ & 6 & 0 & 5 & $\mathbb{1}^{2}$ & $\begin{array}{l}\text { All had experienced a } \\
\text { psychotic episode }\end{array}$ \\
\hline Boper at ol 2002 & 7 & 7 & 1 & 5 & $1^{2}$ & \\
\hline Clarke et al. 1998 & 6 & 5 & 3 & $\mathbb{1}$ & 1 & $\begin{array}{l}\text { Includes participant } 1 \text { in } \\
\text { Eoer et al. }\end{array}$ \\
\hline Verlioeven ed ol "1998 & 6 & $/ 4$ & 1 & 3 & 0 & \\
\hline Deardsmore et al 1990 & 5 & 3 & $2(1)$ & $1(2)$ & 0 & $\begin{array}{l}\text { Inchudes participant } 6 \text { and } 9 \\
\text { in Boer at ot b }\end{array}$ \\
\hline Watanabe et af 1997 & 1 & 1 & 0 & 1 & 0 & Psychotic disorder \\
\hline Whattaker 1997 & 1 & 1 & 0 & 2 & 0 & Psychotic illness \\
\hline
\end{tabular}

of PWS such as skin picking, skill with jigsaw puzzles and high pain threshold. Roof et al. (2000) found that people with disomy had significantly higher verbal IQ scores than those with deletion, but that performance IQ scores did not differ between the two groups (see chapter 1.5).

If this observation of the relationship between the rarer chromosome form of PWS and severe psychiatric illness were to be confirmed by further studies, it would have significant implications both for people with PWS, and for our understanding of the possible genetic basis of such psychiatric disorders and the chromosomal site for a putative candidate gene for psychotic disorder. The increased risk of severe psychiatric disorder in those with the disomy form of PWS may be due to the effect of matemally or paternally imprinted genes located on chromosome 15 but outside the PWS critical region. Thus, in the case of maternally imprinted genes on chromosome 15 (but outside the PWS critical region), neither allele would be expressed, and for any paternally imprinted genes, both alleles would be expressed, rather than only one.

In our study the affective and psychotic illnesses all started in adult life, although it is clear that the onset can be in early adolescence (see Vogels et al. 2003; Verhoeven et al. 2003a; and chapter 8.6). This is consistent with the understanding of psychotic illness as a predominately adult disorder. However, it raises the hypothesis that imprinted gene(s) on chromosome 15 may normally be switched on or off in adolescence or early adult life. A search for imprinted genes and investigation of 
MRNA expression, comparing expression patterns of adults with PWS with different chromosomal types of PWS, would therefore be of considerable interest. The $15 q 13-q 15$ region is a promising region for the phenotype for functional psychosis (Craddock and Lendon, 1999; Leonard ef al. 2002). We were not able to find any articles on Angelman syndrome and psychosis, although this would be difficult to diagnose in view of the severity of the Mental ratardacion in $A S$. Further research regarding the genetics of the non-deletion area of Chromosome 15 (including the effect of imprinting) may be of benefit not only for people with PWS, but also improve our understanding of the genetics of psychotic disonder in general.

Our finding, that psychotic disorder in PWS is associated with UPD was confirmed by Vogels et al. (2003) and Verhoeven et al. (2003a and 2003b). Vogels et al found that out of 59 patients a genetic diagnosis of PWS was confimed in 51 ; six of these had suffered a psychotic episode, with an onset bewween 13 and 19 years. They found that out of these six patients, five had UPD and one had an imprinting centre defect. A further two patients with UPD, and one with an imprinting centre defect did not have a psychotic disorder. Of the other patients, 37 had deletions, one had a translocation and in four patients the exact abnomality could not be determined. These results were unlikely to be the result of a chance finding. Vogels et al. comment that an abnomal imprinting pattern of genes might lead to development of psychotic illness in PWS. Verhoeven et al (2003a) described 23 patients who had been referred for psychiatric evaluation and who were diagnosed with either a relapsing atypical psychosis (18) or a bipolaraffective disorder (5), and were successfully treated with mood stabilisers (lithium and/or valproic acid). In 16 patients they were able to make a detailed genetic diagnosis; of these two had a deletion, and 14 a UPD. They conclude that PWS caused by UPD is associated with psychotic disorder, and they argue that in many patients a diagnosis of atypicall bipolar disorder is appropriate. Verhoeven et al comment that imprinted genes outside the PWS critical region may contribute to the phenotype. Mood swings (as opposed to affective psychosis) are common amongst both those with deletions and UPD, and these appear not to be related to the risk of psychotic illness, but may represent a shift in liability threshold (Holland ef al. 2003 a).

The finding that those with chromosome 15 UPD are likely to develop an affective disorder in early adulthood, and that those with chromosome 15 paternal deletions do not appear to be significantly more likely to develop a psychotic illness than others with a learning disability, indicates that it is the maternal disomy that carries the risk of psychotic illness, and not the syndrome of Prader-Willi per se. It is proposed that the effect on the expression of matermally and patemally imprinted genes of having a maternal UPD of chromosome 15 is a likely explanation. The genetic mechanism is likely to be either the insufficient expression of a matemally imprinted gene or an excess of expression of a paternally imprinted 
gene. This clearly has important implications for the search for possible causes of genetic vulnerability of psychotic illness in the general population.

\subsection{Prader-Willi Syndrome and event related brain activity}

Our findings in the two studies on event-related potentials in people with chromosome 15 deletion and UPD (chapters $7 \mathrm{a}$ and 7 b) support previous research that there are significant cognitive differences between the two groups, with the deletion subgroup functioning wore in the visual tasks and the UPD subgroup performing worse in the auditory tasks. Veltman et al. (2004) found that people with PWS due to UPD had difficulty with one aspect of visual perception, i.e. integrating parts of a perceptual array into a unified construct. Veltman et al. proposed that it is possible that, as they had found that those with UPD had more symptoms suggestive of autism, one explanation for this would be that such a bias towards local rather than global aspects of visual processing is also found in autistic spectrum disorders. This would also be a possible explanation for the finding of better visual memory found in those with UPD compared with deletion, as specific skills in visual memory (attention for details) is also found in some people with autistic trajts.

Our finding, that the UPD group had lower scores on performance, and slower responses than both the deletion and the control groups during the CPT-AX tasks (cesting responses to inhibition tasks, i.e. knowing when nof to press a button) needs further exploration, as similar findings have been found in other groups of patients, such as those with attention deficit hyperactivity disorder and schizophrenia (Strandburg ef al. 1994; Strandburg ef al. 1996). Further research is warranted.

\subsection{Concluding comments and future research}

It is dificult to overestinate the impact of PWS on both the patients themselves and their carers. The eating disorder leaves the patient in an almost constant state of starvation, and the behavioural problems cause significant difficulties for both patients and carers.

A number of articles quoted in this study, and personal experience suggest that people with PWS have a higher quality of living and lower body-mass indices when living in specialist community accommodation for people with PWS, as opposed to non-PWS community accommodation. Studies in order to investigate whether this hypothesis can be sustained would be useful. 
Future research into the prevalence of PWS should attempt to look at larger populations, and it would be useful to combine studies in different countries in order to increase numbers. Longitudinal studies may be useful in order to establish mortallity rates, and causes of death.

In our study on cognitive abilities in PWS we hypothesised that there may be genetic effects on cerebral lateralisation that could account for cognitive differences between genetic sub-types, and we suggested that further studies should investigate the influence of parental cognitive abilities, sex homone levels, and other measures of cerebral dominance to try and establish whether this hypothesis is correct.

Much research on PWS has concentrated on problem behaviour, and further research, investigating positive behavioural characteristics, would allow profes-sionals to present a more accurate and rounded picture of the behavioural profile associated with PWS. Longitudinal studies would help to clarify changes in behaviour over time.

Further, and larger studies on psychosis in PWS are clearly needed, including detailed analyses of the psychopathology and genetic abnomalities. Detailed psychiatric and genetic examination of any patient older than 28 years with PWS caused by disomy who does not develop psychotic symptoms could provide valuable clues into the aetiology of psychosis in both PWS as well as in the general population. It may also be useful to attempt to establish whether there is any evidence of psychotic symptoms in people with Angelman Syndrome.

Recently, Holland et al. (2003b) proposed that one mechanism could account for the main infant and childhood phenotypes of PWS including the underlying growth and sex hormone deficiencies, the abnormal satiety response to food intake, and the arrested brain development. These phenotypes could then be explained by the failure of expression of one paternally expressed gene. Testing this hypothesis, based in part on the research presented in this study, should be a key objective of future studies with people with PWS.

\section{References}

Beardsmore A., Dorman T., Cooper S.-A. \& Webb T. (1998) Affective psychosis and Prader-Willi syndrome. Joumal of Intellectual Disability Riseardh 42, 463-71.

Boer.H., Holland A., Whittington]., ButlerJ., Webb T. \& Clarke D. (2002) Psychotic illness in people with Prader Willi syndrome due to chromosome 15 matemal uniparental disomy. Lance 359, 135-6.

Boer H., Stauder J.E.A., Gerits R.H.A., Tummers A., Whitington J., Webb T. \& Curfs L.M.G. Prader-Willi syndrome: Behavioural differences between deletion and disomy subtypes. In preparation. 
Casidy S.B., Forrythe M. Heeger S. Nicholls R.D., Schork N., Benn P. \& Schwartz S. (1997) Comparison of phenotype between patients with Prader-Willi syndrome due to deletion $15 q$ and umiparental disomy 15. American joumal of Medical Genetics 68, 433-40.

Clarke D.J. \& Boer H. (1998) Problem Behavion Associated wich Delecion Prader-Willi, Smith-Magenis, and Cri Du Chat Syndromes. American Journal on Mental Retardation $103,264-71$.

Clarke D., Boer H., Webb T., Scott P., Frazer S., Vogels A., Borghgraef M. \& Curfs L M. G. (1998) Prader-Willi syndrome and psychotic symptoms 1. Case descriptions and genetic studies. Joumal of Intellecual Disability Researh $42,440-50$.

Clarke D.J. Boer H., Whittington J., Holland A., Butler J. \& Webb T. (2002) Prader-Will syndrome, compulsive and ritualistic behaviours: the first population-based survey. British Jounal of Psychiary 180, 358-62.

Cooper S.A. \& Collacott R.A. (1996) Depressive episodes in adults with learning disabilities. Insh Joumal of Psychalogical Medicine 13, 105 13.

Collacon R.A., Cooper S.-A. \& McGrother C. (1992) Differential rates of psychiatric disorders in adults with Down's syndrome compared with other mentally handicapped adults. British joumal of Psychatry 161, 671-4.

Corbett J.A. (1979) Psychiatric morbidity and mental retardation. In: Psychiatric Mlunes and Mental Handiap (eds F. E. James \& R. P. Snath), pp.11-25. Gaskell, London.

Craddock N., Lendon C. (1999) Chromosome Workshop: 11, 14 and 15. Anerican Journal of Medical Genetio (Newropsychanic Genetics) 88, 244-54.

DelParigi A., Tschöp M. Heiman M.L., Salbe A.D., Vozarova B., Sell S.M., Bunt J.C. \& Tataranni P.A. (2002) High circulating ghrelin: a potential cause for hyperphagia and obesity in Prader-Willi syndrome. Joumal of Clinical Endacrinology and Metabolssn 87, $5461-4$

Dykens E.M. (2004) Maladaptive and compulsive behavior in Prader-Willi syndrome: New insights from older adults. American Joumal on Mental Retardation 109, 142-53.

Dykens E.M. \&. Smith A.C.M. (1998) Distinctiveness and correlates of madaptive beluaviour un children and adolescents with Snith-Magenis syndrome. Joumal of Inellertual Disability Rescand 42, 48:-9

Gillesen-Kacshach G., Robinson W., Lohmann D., Kaya-Wescerloh S., Passarge E. \& Horsthemke B. (1995) Genotype-phenotype correlation in a series of 167 deletion and non-deletion patients with Prader-Willi syndrone. Human Genefics 96, 638-43.

Goldstone A. P., Thomas E.L., Brynes A.E., Castroman G., Edwards R., Ghatei M. A., Frost C., Holland A.J., Grossman A.B., Korbonits M., Bloom S.R. \& Bell J.D. (2004) Elevated tasting plasma ghrelin in Prader-Willi syndrome adults is not solely explained by their reduced visceral adiposity and insulin resistance. The Joumal of Climical Endocrinology and Motiblism 89, 1718-26.

Goldstone A.P., Ummehopa U.A.,Bloom S.R. \& Swab D.F. (2002) Hypothalamic NPY and agouti-related protein are increased in human illness but not in Prader-Willi syndrome and other obese subjects. The Joumal of Chinel Endocrinology and Metabolisn 87, 927-37. 
Greenswag L.R. (1987) Adults with Prader-Wilh syndrome: a survey of 232 cases. Dovelopmental Medicine and Child Neurology 29,637-48

Holland A.J., Whittington J.E., Butler J., Webb T., Boer H. \& Clarke D. (2003a) Behaviournl phenotypes associated with specific genetic disorders: evidence from an population-based study of people with Prader-Willi syndrome. Pychological Medicine 33, 141-53.

Holland A.J, Whittington J.E. \& Hinton E.C. (2003b) The paradox of Prader-Willi syndrome: a genetic model of starwation. Lancet 362, 989-91.

Leonard S., Gault J., Hopkins J., Logel J., Vianzon R., Short M, Drebing C., Berger R, Venn D., Sirota P., Zerbe G., Olincy A., Ross R.G., Adler L.E. (2. Freednan R. (2002) Association of promoter variants in the alpha 7 nicotinic acetylcholine receptor subunit gene with an inhibitory deficit found in schizophrenia. Ardives of General Psydiatn 59, 1085-1096.

Nyhan W.L. (1995) Foreword. In: Behavioural Phenotypes (eds G. O'Brien \& W. Yule) Mac Keith Press, London.

O'Brien G. (2002) The clinical relewance of Behavioural Phenotypes. In: Behavioural Phenoivpes in Climical Practice (ed G. O'Brien) Mac Keith Press, London.

O'Brien G. \& Yule W. (1995) Why behavioural phenotypes? In: Behawoural Phenotypes (eds G. O'Brien \& W. Yule) Mac Keith Press, London.

Roof E., Stone W., MacLean W., Feurer I.D., Thompson T. \& Butler M.G. (2000) Intellectual characteristics of Prader-Willi syndrone: comparison of genetic subtypes. Joumal of Intellectual Disability Research 44, 25-30.

Schrander-Stumpel C.Th.R.M., Curfs L.M.G., Sastrowijoto P. Cassidy S B, Schander A.J.P. \&. Fryns J.P. (2004) Prader-Willi Syndrome: Causes of Death in an Intemational Series of 27 Cases. American Jommal of Medical Genetics 124, 333-338.

Smith A., Egan J., Ridley G., Haan E., Montgomery P., Williams K. \& Ellott E. (2003) Birth. prevalence of Prader-Willi syndrome in Australia. Archives of Disense in Childhood 88 , $263-4$.

StauderJ.E.A., Boer H., Gerits R.H.A., Tummers A, Whittington J. \& Curfs L.M.G. Marked differences in behavioural phenotype between parental deletion and maternal uniparental disomy in Prader-Willi syndrome: Behaviou and event related brin activity to a response imhibition task. Submitted.

Strandburg R.J., Marsh J.T., Brown W.S., Asamow R.F. \& Guthite D. (1994) Information-processing deficits across childhood- and adult-onset schizophrenia. Sohizophronia bulletin $20,685-95$.

Strandburg R.J., Marsh J.T., Brown W.S., Asarnow R.F., Higa J., Harper R., \& Guthrie D. (1996). Continous- Processing-Related Event-Related Potentials in children with Atten-tion Deficit Hyperactivity Dionder Biological Psychiary 40, 964-980.

Swab D.F., Purba J.S. \& Hofman M.A. (1995) Alterations in the hypothalamic paraventricular mucleus and its oxytocin neurons (putative satiety cells) in Prader-Will syndrone: a study of frve cases. The Joumat of dimian endorinology and metabolism 80, 573-9.

Veltman M.W.M., Thompson R.J., Roberts S.E., Thomas N.S., Whittington J. B Bolton P.F. (2004) Prader-Willi syndrome - a study comparing deletion and uniparcontal disony cases with reference to autism spectrum disorders. European child G adolesentysychiary $13,42-50$. 
Verhoeven W.M.A., Curfs L.M.G. \& Tunier S. (1998) Prader-Willi syndrome and cycloid psychoses. Joumal of Jntellemal Disability Researh $42,455-62$.

Verhoeven W.M.A., Tuiner S. Curs L.M.G. (2003a) Prader-Willi syndrome: the psychopathological phenotype in uniparental disony. Joumal of medical genetics: 40, e112.

Verhoeven W.M.A, Tumier S. \& Curfs L.M.G. (2003b) Prader-Willi syndrome: Cycloid. psychosis in a genetic subtype? Adta Neuropsychiatrica 1.5, 32-7.

Vogels A., Matthijs G., Legius E., Devriendt K. \& Fryns. J. (2003) Chromosome 15 maternal uniparental disomy and psychosis in Prader- Willi syndrome. Joumal of medical genetics 40 , $72-3$.

Vogels A., Van Den Ende J., Keymolen K., Mortier G., Devriendt K., Legius E. \& Fryns. J.P. (2004) Minimum prevalence, birth incidence and cause of death for Prader- Willi syndrome in Flanders. Etwopean jowrtal of hwman genetics 12, 238-40.

Watanabe H. Olmoni O. \& Abe K. (1997) Recurrent brief depression in Prader-Willi syndrome: A case report. Psychiatric Genetic 7, 41-4.

Whittaker J.F, Cooper C., Harrington R. C. \& Price D.A. (I997) Prader-Willi syndrome and acute psychosis. International Joumal of Pychiatry in Clinical Practice 1, 217-9.

Whittington J.E., Holland A.J., Webb T., Butler J.V., Clarke D.J. \& Boer H. (2001) Population prevalence and estimated birth incidence and mortality rate for people with Prader-Willi Syndrome in one UK Health Region. Jownal of Modical Genetic 38, 792-8.

Wigren M. \& Hansen S. (2003) Rituals and compulsivity in Prader-Willi syndrome: profile and stability. Jowrnal of infellectual disability research $47,428-38$. 


\section{Summary}

The study of patients with Prader-Willi Syndrome (PWS) offers an opportunity to relate specific behaviours to defined genetic abnomalities. PWS is associated with an absence of a paternal contribution to a defined area of chromosome 15 . The syndrome is characterised by hypotonia and hypogonadism at birth, and extreme hyperphagia which starts in early childhood. Most adults with PWS are overweight, which can result in a reduced life expectancy. Certain behavioural and personality characteristics (including obsessive-compulsive symptoms and psychosis) appear to be common in PWS and we set out to discover whether these behaviours could be explained through other means, such as the fact that most people with PWS have mental retardation (learning disability), and many are obese.

Chapter 1 aims to give an overview of the up to date knowledge of PWS that led to the research presented in this thesis. Chapter 1.2 summarises the clinical presentation of PWS, comparing the original study on PWS with later studies, and commenting on how the discovery of the genetic causes of PWS led to a different approach to diagnosing the disorder.

In Chapter 1.3 previous studies on the epidemiological clata on PWS are discussed. As previous epidemiological studies involved relatively small populations, and were done before genetic testing became widespread, we attempted to estimate the population prevalence, the birth incidence and mortality rates of people with PWS in a population of 5 million people, using confirmed genetic diagnoses where possible (Chapter 2). We found a population prevalence of at least 1:52000, a birth incidence of at least $1: 29000$ and an overall death rate of about $3 \%$ per year. Further population studies have found very similar birth incidences (see chapter 8.2).

Chapter 1.4 gives a brief summary on the genetic features of the syndrome, including a description of the phenotypically different, but genetically similar Angelman Syndrome, and the different genetic causes of PWS including deletion, uniparental disomy and imprinting defect.

Chapter 1.5 summarises previous studies on the cognitive abilities of people with PWS, including the finding that cognitive performances differ between the two main (deletion and uniparental disomy) genetic subgroups of PWS. In our study (Chapter 3a) we therefore attempted, in a population sample, to establish what mechanisms can explain the distribution of cognitive ability in those with PWS 
and to find whether there were any systematic cognitive differences between the deletion and UPD subgroups of PWS. We found that the population sample had an approximately normal $\mathrm{IQ}$ distribution, and that the mean IQ was 40 points below that of the general population. The cognitive profiles of those with disomies differed from both those with deletions and the comparison LD group (the latter two groups were very similar) in terms of better verbal abilities and impaired coding ability, and some people with PWS deletions were found to have strong visuo-spatial skills. In our study on academic underachievement in PWS (Chapter 3b), we examined the relationship between cognitive abilities of people with PWS and academic achievement in reading, writing and arithmetic, using a comparison group of people who had been thought to have PWS, but were found not to have the disorder, together with a group of people with learning disabilities not caused by PWS. The aims of this study were to investigate whether attainments which people with PWS might have been expected to achieve on the grounds of their $1 \mathrm{Q}$ were achieved, and to ask which factors might be associated with any underachievement. We found that those who attended special schools achieved relatively lower academic scores than those who attended mainstream schools, and we hypothesise that this may be associated with children with PWS with emotional and behavioural problems being more likely to be placed in special schools, and we propose that a fallure to recognise and address the specific educational needs that associated with this combination of poor socialisation skills and complex maladaptive behaviours may explain these findings.

An overview of previous studies on behavioural studies of people with PWS is given in chapter 1.6. Before the mid-1990s few studies had used screening instruments or standardised measures of behavioural abnormalities or personality among individuals with PWS. We therefore used a widely used standardised instrument (the Aberant Behavioral Checklist) to compare maladaptive behaviours of adults with PWS with those of adults with learning disability using social service and voluntary sector community residential facilities. This study is presented in Appendix 1 , and concludes that temper tantrums, self-injury, impulsiveness, lability of mood, inactivity and repetitive speech are characteristic behaviours in PWS in adult life. In two further studies we used standardised instruments to compare the behaviour of patients with PWS with patients with other genetic syndromes. In Chapter $4 a$ we again used the Aberrant Behavior Checklist to examine problem behaviours in individuals who had one of three chromosome deletion disorders (cri du chat syndrome, Prader-Willi syndrome caused by deletion, and Smith-Magenis syndrome). We found that the results relating to Pader-Willi syndrome were largely consistent with previous research using other methodologies, and that all three chromosome deletion disorders are associated with greater ratings of severity of problem behaviours than the comparison groups on at least one sub-scale of the checklist. Our study on the development and 
behaviour in a sample of 210 children and adults with Prader-Willi syndrome (PWS) is presented in Chapter 4b. In this study we used the Society for the Study of Behavioural Phenotypes Postal Questionnaire, a 98 question structured interview with 8 developmental domains and 10 behavioural domains. Adults with PWS had more problems than children with daytime sleepiness and other sleep probm lems, unusual routines, and self-injury; and fewer problems with faddy eating. verbal abuse and stubbornness. Changeable mood was a problem for both adults and children; adults had more problems with Low mood. The Society for the Study of Behavioural Phenotypes Postal Questionnaire was also used on a sample of patients with Rubinstein-Taybi syndrome, which is associated with microdeletions at $16 \mathrm{p} 13.3$, and this study is presented as Appendix 2 in order to allow comparison with chapter $4 \mathrm{~b}$.

Chapter 1.7 includes a brief review of the literature on compulsive and ritualistic behaviours in those with PWS. No population based research, and no research with an appropriate control group had been done, which led to our research presented in Chapter 5. We found that PWS was associated with high rates of ritualistic behaviours such as the need to ask or to tell something, insistence on routines, hoarding and ordering objects, and repetitive actions and speech, compared to a control group of people with learning disability and high body-mass indices. Typical obsessive-compulsive symptoms such as checking, counting and cleaning compulsions, or obsessional thoughts, were not found in the PWS group.

Chapter 1.8 summarises previous studies on PWS and psychotic symptoms. In order to confirm whether psychotic symptoms are more common than expected in people with PWS, six people with PWS and psychosis are described in Chaprer $6 a$. We concluded that it was likely that there was an association between PWS and psychotic symptoms, although in this paper no correlation with a single genetic abnormality was found. In Chapter 66 we were able to investigate the relation between genetic subtypes of the syndrome and psychiatric morbidicy in a population-based study of PWS. We found that psychotic illness was associated with PWS caused by disomy, but not with PWS caused by delerion of chromosone 15. This finding was subsequently confrmed by further studies (sec Chapter 8.6). Chapter 1.9 gives an introduction to the study of event-related potentials in people with PWS. Recordings of event-related brain activity was used to examine whether the striking differences between the genetic subtypes, such as the heightened vulnerability of PWS patients with uniparental disomy for affective psychoses, and the dissimilar cognitive profilles were supported by psycho-physiological studies (Chapter 7a and Chapter 7b). In the first study (chapter 7a) we found that those with PWS caused by uniparental disomy had lower performance and slower reaction times than those with $\mathrm{PW}$ W caused by deletion and than a control group in a response inhibition task. These psycho-physiological data support the 
psychological findings of differences in phenotype between parental deletion and uniparental disomy PWS subgroups. In the second study (chapter 7b) we concluded that those with deletion have relatively better visual skills, and those with UPD relatively better auditory skills. We also found some support for the previous finding of high scores on items relating to autistic spectrum disorder in. PWS.

The discussion (Chapter 8) compares the findings in this thesis with other studies, and concludes that the behaviours associated with PWS constitute at least one classical behavioural phenotype with the genetic subtypes (deletion and uniparental. disomy) resulting in subtly different behavioural phenotypes. A number of suggestions are made regarding future research. 


\section{Samenvatting}

Onderzoek aan patienten met het Prader-Willi Syndroom (PWS) makt het mogelijk relaties aan te tonen tussen specifiek gedrag en genetische afwijkingen. PWS gat samen met een afwezigheid van de patemale contributie aan een bepaald gebied van chromosoom 15 (15q11-13). Het syndroom wordt onder andere gekarakteriseerd door neonatale hypotonie en hypogonadisme en extreme hyperfagie beginnend in de vroege kinderjaren. De meeste volwassenen met PWS hebben overgewicht, hetgeen kan resulteren in een gereduceerde levenswerwachting. Bepaalde gedrags - en persoonlijkheidseigenschappen, dwangneurotische symptomen en psychosen blijken vak voor te komen in PWS. In dit proefschrift wordt geprobeerd te bepalen of dit gedrag andere oorzaken kan hebben, zoals het feit dat de meeste mensen met PWS een verstandelijke handicap en overgewicht hebben.

In hoofdstuk 1 wordt een overzicht gegeven van de kennis van PWS die tot het onderzoek wan dit proefschrift leidde. In paragraaf 1.2 wordt de klinische presentatie van PWS samengevat en een vergelijking gemakt tussen de originele studie over PWS en recentere studies. In paragraaf 1.2 wordt ook besproken hoe de ontdekking van de genetische oorzaken van PWS tot een verunderde benadering van het diagnosticeren van het syndroom leidde.

In paragraaf 1.3 worden eerdere studies over de epidemiologische gegevens over PWS besproken. Vorige epidemiologische studies beschreven vrij kleine populaties en vonden plaats vóór genetische testen wijdverspreid werden gedan. Een schatting werd gemakt van de prevalentie, de geboorte-incidentie en hetstertecijfer wan mensen met PWS in een bevolking van 5 miljoen mensen, war mogelijk gebruik makend van genetische diagnoses (hoofdsuk 2). Een prevalentie van minstens 1:52000, een incidentie van minstens 1:29000 en een sterfteciffer van ongeveer $3 \%$ per jaar werd gevonden. Hieropvolgende bevolkingsstudies vonden zeer vergelijkbare geboorte-incidenties (zie paragraaf 8.2).

In paragraf 1.4 wordt een korte samenvatting gegeven van de genetische eigenschappen van het syndroom, met inbegrip van een beschrijving van het phenotypisch verschillende, maar genetisch overeenkomstige Angelman Syndroom en de verschillende genetische oorzaken van PWS met inbegrip van deletie, uniparentele disomie en imprinting defect.

In paragraf 1.5 worden eerdere studies samengewat over de cognitieve capaciteiten van mensen met PWS, met inbegrip van de bevinding dat de cognitieve 
prestaties tussen de twee meest voorkomende (deletie en uniparentele disomie (UPD) genetische subgroepen van PWS verschillen. In hoofdstuk $3 a$ werd daarom geprobeerd om, in een bevolkingssteekproef, vast te stellen welke mechanismen de distributie van cognitieve capaciteiten in mensen met PWS kunnen verklaren en uit te vinden of er tussen de deletie- en UPD subgroepen van PWS systematische cognitieve verschillen waren. Het IQ in de bevolkingssteekproef bleek een ongeveer normale distributie te hebben, met een gemiddelde $1 \mathrm{Q} 40$ punten onder dat van de totale bevolking. De cognitieve profielen van de groep met disomieën verschilden van zowel die met deleties als van de vergelijkingsgroep met verstandelijke handicap (de laatstgenoemde twee groepen waren zeer vergelijkbaar) in termen van betere mondelinge capaciteiten en verminderd coderend vermogen. Sommige mensen met PWS veroorzaakt door deletie bleken sterke visuo-spatiële vaardigheden te hebben. In het onderzoek naar het academisch onvoldoende presteren in PWW (hoofdstuk 3b), werd het verband onderzocht tussen cognitieve capaciteiten van mensen met PWS en schoolprestaties in lezen, schrijven en rekenkunde, gebruik makend van een vergelijkingsgroep van mensen waarvan eerst verondersteld werd dat ze PWS hadden, maar waarvan later bleek dat ze het syndroom niet hadden, en met een groep van mensen met verstandelijke handicap dat niet door PWS werd veroorzaakt. De doelstellingen van deze studies waren om te onderzoeken of de vaardigheden werden bereikt die van mensen met PWS zouden kunnen worden verwacht in verband met hun IQ, en om te bepalen welke factoren met het onvoldoende presteren zouden kunnen worden geassocieerd. Mensen met PWS die scholen voor moeilijk lerende kinderen hadden bezocht bereikten relatief lagere academische scores dan diegenen die op gewone scholen hadden gezeten. Dit lijkt samen te hangen met het feit dat kinderen met PWS die emotionele en gedragsproblemen hebben eerder in speciale scholen worden geplaatst. Verondersteld wordt dat het verzuim om de specifieke onderwijsbehoeften die samengaan met deze combinatie van slechte socialisatievaardigheden en complex onaangepast gedrag te erkennen, deze bevindingen kan verklaren

Een overzicht van eerdere studies over gedrag van mensen met PWS wordt gegeven in paragraaf 1.6. In deze studies werd bijna geen gebruik gemaakt van screeningsinstrumenten of gestandaardiseerde metingen van gedragsafwijkingen of persoonlijkheidskenmerken onder mensen met PWS. Een wijd gebruikt gestandaardiseerd instrument (Aberrant Behavioural Checklist) werd daarom nu gebruikt om onaangepast gedrag van volwassenen met PWS te vergelijken met die van beschermd wonende volwassenen met een verstandelijke handicap. Deze studie wordt gepresenteerd in Bijlage 1 en concludeert dat woedeanvallen, zelfverwonding, impulsiviteit, stemmingslabiliteit, inactiviteit en repeterende spraak. kenmerkend voor het gedrag bij volwassenen met PWS zijn. In twee verdere 
studies werden gestandaardiseerde instrumenten gebruikt on het gedrag van patiènten met PWS met patiënten met andere genetische syndromen te vergelijken. Hoofistuk $4 a$ bevat de resultaten van onderzoek met de Aberrant Behavioral Checklist om probleemgedrag in individuen te onderzoeken die éen van drie deletiesyndromen (cri du chat syndroom, Prader-Willi syndroom veroorzakt door deletie, en Smith-Magenis syndroom) hadden. Deze resultaten met betrekking tot het PWS waren grotendeels vergelijkbaar met eerder onderzoek dat van andere methodologieën gebruik had gemaakt. Alle drie deletiesyndromen bleken samen te gaan met emstiger probleemgedrag dan de vergelijkingsgroepen op minstens één sub-scale van de checklist. Het onderzoek naar de ontwikkeling en het gedrag in een steekproef van 210 kinderen en volwassenen met PWS wordt gepresenteerd in hoofistuk 46 . In deze studie werd gebruik gemakt van de Society for the Study of Bebavioural Phenotypes Postal Questionnaire, een gestructureerd gesprek met 98 vragen over acht ontwikkelingsdomeinen en tien gedragsdomeinen. De volwassenen met PWS hadden meer problemen dan de kinderen met slaperigheid overdag en andere slaapproblemen, ongebruikelijke routines en zelfverwonding; en minder problemen met grillig eten, verbale aggressiviteit en koppigheid. Veranderlijke stemming was een probleem voor zowel volwassenen als kinderen; volwassenen hadden meer problemen met depressieve stemming. De Study of Behavioural Phenotypes Postal Questionnaire werd ook gebruikt bij een steekproef onder patiënten met Rubinstein-Taybi syndroom, dat door microdeletions bij 16p13.3 wordt veroorzakt en deze studie wordt gepresenteerd als Bilage 2 om vergelijking met hoofdstuk $4 b$ mogelijk te maken.

Paragraf 1.7 bevat een kort overzicht van de literatuur over dwangmatig en ritualistisch gedrag bij mensen met PWS. Bevolkingsonderzoek en onderzoek met geschikte controlegroepen werden niet eerder gedaan, hetgeen leidde tot het onderzoek dat in hoofdstwk 5 wordt gepresenteerd. In vergelijking met een controlegroep van mensen met verstandelijke handicap en hoge body-mass indices werd PWS geassocieerd met een hoge mate van ritualistisch gedrag, zoals de behoefte om iets te vragen of te vertellen, het aandringen op routines, hansteren en ordenen van voorwerpen, en herhaalde handelingen en uitlatingen. Typische dwangneurotische symptomen zoals controleren, tel- en schoonmakdwang of dwangmatige gedachten, werden niet gevonden bij de groep met PWS. Paragraf 1.8 vat eerdere studies over PWS en psychotische symptomen samen. In hoofdstuk $6 a$ wordt een aantal mensen met PWS en psychose beschreven. Uit deze studie bleek dat het waarschijnlijk was dat er een samenhang tussen PWS en psychotische symptomen bestond, hoewel in dit arcikel geen aanwijzing werd gevonden dat er een samenhang was tussen psychose en een bepaalde genetische afwijking. In hoofdstuk 6 b wordt de relatie onderzocht tussen genetische subtypes wan het syndroom en psychiatrische morbiditeit van PWS in een bevolkingsstudie. Psychosen bleken geassocieerd te zijn met PWS die door disomie wordi 
veroorzaakt, maar niet met PWS veroorzaakt door deletie van chromosoom 15. Deze bevindingen werden bevestigd door andere studies (zie Paragraaf 8.6).

Paragraaf 1.9 bevat een introductie over onderzoek naar event-related potentials in mensen met PWS. Registraties van event-related hersenenactiviteit werden gebruikt om te onderzoeken of de opvallende verschillen tussen de genetische subtypes, zoals de verhoogde kwetsbaarheid van patiënten met PWS met UPD voor affectieve psychosen en de ongelijke cognitieve profielen door psychofysiologische studies worden gesteund (hoofdstuk $7 a$ en hoofdstuk $7 b$ ). In de eerste studie (hoofdstuk 7a) wordt geconcludeerd dat diegenen met PWS veroorzaakt door uniparentele disomie lagere prestaties en langzamere reactietijden hadden dan diegenen met PWS veroorzaakt door deletie en een controlegroep in een reactie-inhibitietak. Deze gedragsgegevens steunen eerdere psychologische bevindingen van verschillen in fenotype tussen deletie en disomie subgroepen bij PWS. De tweede studie (hoofdstuk 7 b) betreft de analyse van de psychophysiologische gegevens. Hieruit blijkt dat mensen met PWS veroorzaakt door deletie relatief betere visuele vaardigheden, en PWS personen met UPD relatief betere auditieve vaardigheden hadden.

Het geheel wordt afgesloten met een discussie (hoofdstuk 8), waarin de bevindingen van deze thesis vergeleken worden met andere studies. Het is misschien wel gerechtvaardigd om niet te spreken van één klassiek gedragsfenotype, maar van twee verschillende gedragsfenotypes gerelateerd aan deletie en disomie. Een aantal suggesties wordt gedaan over toekomstig onderzoek. 


\section{Dankwoord}

Dit proefschrift is tot stand gekomen door samenwerking. Een groot aantal mensen hebben een grote of kleine bijdrage geleverd en het is deze samenwerking, op verschillende vlakken, die ik het meest heb gewaardeerd in de totstandkoming van dit proefschrift.

Mijn interesse in het Prader-Willi syndroom (PWS) begon toen ik in 1993 als Senior Registrar in Learning Disability (arts-assistent in de tweede helft van de opleiding, specialiserend in verstandelijke handicap) bij David Clarke in Birmingham kwam werken. He told me to have five articles published by the end of that year. Een jaar later, op een conferentie in Maastricht, ontmoette ik mijn latere promotor Leopold Curfs. De exacte datum warop hij mij begon an te moedigen om met de hulp van een aantal artikelen te promoveren is niet meer te achterhalen, maar uiteindelijk ging ik er zelf ook in geloven. I $\mathrm{k}$ wil Professor Curfs hierbij hartelijk danken voor het vertrouwen, de steun en met name ook het geduld (en het feit dat hij de goede middenweg vond tussen duwen en trekken), want het was niet altijd makkelijk, om naast mijn drukke baan voldoende tijd te vinden voor het voltooien van de verschillende projecten. Mijn dank gaat ook uit naar Professor Geraedts voor zijn constructieve opmerkingen en naar Judith Maszewski voor haar praktische steun.

In Birmingham I would like to thank in particular DrDavid Clarke for his enthusiasm, support and friendship. His influence can be seen throughout this thesis, and in many ways this thesis can be seen as a sequel of his $M D$. Thanks also to Professor John Corbett, who made those who trained with him feel that they had something real to contribute. I have been very lucky indeed to have been involved in the Cambridge PWS population study with Tony Holland, Joyce Whittington, Tessa Webb, Jill Butler, and David Clarke. It was again the collaboration and the sharing of ideas which made this special. I would especially like to thank Tony Holland for his support and encouragement following our finding that psychosis was associated with disomy in PWS. I feel honoured to have been able to work so closely with him. Joyce I would like to thank for her patience and kindness, and also for her help in getting the evoked potential studies started. The cvoked potential studies may never have happened without the initiative and organizational skills of Yvonne Wood, my secretary for many years. It is no wonder that she was offered a much more responsible job within our trust.

Rolf Gerits en Anke Tummers, psychologiestudenten in hun laatste jaar, kwamen in Februari 2003 naar Engeland om met het onderzoek op evoked potentials te 
helpen. Zij kwamen met een aanbeveling van Leopold Curfs, en Rachel, Alyssia en ik hebben nog steeds hele goede herinneringen aan de twee weken dat ze bij ons in Tanworth verbleven. Mijn dank gaat ook uit naar Hans Stauder, voor de samenwerking.

Over the last eleven years I have worked with many people with PWS, their families and their carers. I have been struck by the willingness of many of those suffering from PWS to give up some of their time, in the hope of, ultimately, helping others with the disorder. I would like to thank those in the PWS Association (UK) and in particular Jackie Waters for their support. I am also grateful to those at Gretton Homes, a specialist provider for PW'S. Maurice Reynolds, and all the staff working with him as well as the residents have always made me feel welcome, and it was a pleasure to be able to visit the homes. Mijn dank gaat ook uit maar de Nederlandse Prader-Willi oudervereniging.

Whilst training as a junior psychiatrist in Exeter I was encouraged to be involved in academic research, and I would like to thank Martin Briscoe, my first trainer, for his encouragement and infectious optimism. I would also like to thank my friend Deborah Reed, $\mathrm{PhD}$, who kindly pointed out that it would be helpful for my first attempt at an academic article to have a beginning, a middle and an end.

Ek is baie dankbaar vir Annemie Bosch en haar man David Bosch, eermaals professor in teologie aan die Universiteit van Suid Afrika. Die paar maande wat ek as kind by hulle in Umtata gewoon het (in plaas van die kosskool) sal ek nooit vergeet nie. Oom David was vir my as kind 'n inspirasie, en sy onverwagte dood in 1992 is nog steeds vir my swar. Oom David was amper altyd besig met ondersoek of onderrig, maar as hy nie gewerk het nie was hy volledig daar vir ander mense. Tannie Annemie het my geleer dat dit lekker voel om hard te werk vir skool. Ik ben dankbaar voor de vriendschap die ik vond op de Vereniging voor Christelijke Studenten te Amsterdam, en in het bijzonder voor Jannet van der Spek (schoonzus) en Jaap Neerken, die toegestemd hebben paranimf te willen zijn. Alhoewel Pierre Eijgenraam nooit op de Vereniging heeft gezeten, was hij er zo bij betrokken dat het niets gaf. Pierre, Jaap en Jannet, ik heb bijzonder veel steun aan jullie gehad.

I would like to thank my friends in Tanworth for their interest (for instance in "proud of your willy syndrome") and in particular Judge Marten Coates, for his support.

I would also like to thank the Terrell family in Cornwall for their support and encouragement. At my first audience during a national conference I was able to break the ice by saying that my father-in-law had fallen asleep whilst I was practicing my talk on him. Many weekends in Comwall were spent on research.

De familie Boer dank ik voor hun steun in de afgelopen jaren. Mijn vader, die verder nooit adviezen placht te geven, sprak mij toen ik zestien of zeventien jaar was serieus toe en zei dat ik warschijnlijk gelukkiger zou zijn als arts dan als 
theoloog. Of dat waar was weet ik niet, maar ik was warschijnlijk nooit een tevreden dominee geworden. Mijn moeder heeft nooit blijk gegeven van enige twijfel dat het met mij wel goed ging komen. Erik bedank ik voor zijn steun en trouw, Eefke voor haar liefde en zorgzaamheid, en mijn jongere broers W/arner en Henk voor hun interesse over de jaren.

Dit proefschrift zou nooit tot stand zijn gekomen zonder de steun van mijn lieve vrouw Rachel. During the times that I have worked on and worried about this thesis you have supported me in ways that are probably not fully clear even to you. Thank you. 



\section{Curriculum Vitae}

Gezinus Harm Boer (Harm Boer) werd op 22 augustus 1959 geboren te Delft. Hij bracht een deel van zijn jeugd door in Nieuw Guinea en Zuid Afrika, en behaalde in 1978 zijn VWO diploma te Maastricht. Hij studeerde aan de Vrije Universiteit. in Amsterdam; eerst theologie, maar na twee weken begon hij aan de studie geneeskunde, die hij in 1986 afrondde. Daarna werkte hij als onbezoldigd arts bij Kruispost, een door de Amsterdamse Kerkenraad gesteunde eerstehulppost voor mensen zonder verzekering en tegelijkertijd als bezoldigd groepsleider bij een gezinsvervangend tehuis van de Stichting Philadelphia Voorzieningen.

In 1987 verhuisde hij naar Engeland, waar hij werkte als House Officer (jonge assistent) in chirurgie en interne geneeskunde en een jaar later begon hij in Exeter aan de opleiding psychiatrie. Hij werd in 1993 een member of the Royal College of Psychiatrists en specialiseerde zich in Birmingham in de psychiatrie voor mensen met een verstandelijke handicap en in de forensische psychiatrie. Sinds 1997 is hij als specialist in de forensische psychiatrie voor verstandelijk gehandicapten verbonden aan de Janet Shaw Clinic, een Medium Secure Unit (gesloten afdeling; vergelijkbaar met een TBS Kliniek) in Birmingham. Ook is hij betrokken bij de Cambridge Prader-Willi Syndrome Study. 


\section{Publications}

Upton M., Boer H. \& Neale A. (1994) Patients or clients? A hospital survey. Psychiatric Bulletin 18, 142-3.

Clarke D., Boer H. \& Webb T. Genetic and behavioural aspects of Prader-Willi Syndrome. (1995) Mental Handicap Research 8, 38 - 53.

Clarke D., Boer H., Chung M.C., Sturmey P. \& Webb T. (1996) Maladaptive behaviour in Prader-Willi Syndrome in adult life. Joumal of Intellectual Disability Research 40, 159-65.

Boer H., Booth N., Russell D., Powell R. \& Briscoe M. (1996) Antidepressant prescribing prior to suicide: role of doctors. Psychiatric Bulletin 20, 282-4,

Boer H., Clarke D., Webb T., Holland A. \& Curfs L.M.G. (1998) Het Syndroom van Prader-Willi: van gen naar gedrag (Prader-Willi Syndrome: From gene to behaviour). Tijdschrift voor Psychiatrie 40, $108-14$.

Clarke D.J. \& Boer H. (1998) Problem behaviors associated with deletion Prader-Willi, Smith-Magenis, and Cri du Chat syndromes. American Journal on Mental Retardation 103, 264-71.

Clarke D., Boer H., Webb T., Scott P., Frazer S., Vogels A., Borghgraef M. \& Curfs L.M.G. (1998) Prader-Willi syndrome and psychotic symptoms 1. Case descriptions and genetic studies. Jommal of Intellectual Disability Research 42, $440-50$.

Boer H. \& Clarke D. (1999) Development and behaviour in genetic syndromes: Prader-Willi Syndrome. Joumal of Applied Research in Intellectual Disabilities 12, $294-301$.

Boer H., Langton J. \& Clarke D. (1999) Development and behaviour in genetic syndromes: Rubinstein-Taybi syndrome. Joumal of Applied Researh in Intellectual Disabilities 12, 302-7. 
Whittington J.E., Holland A.J., Webb T., Butler J.V., Clarke D.J \& Boer H. (2001) Population prevalence and estimated birth incidence and mortality rate for people with Prader-Willi Syndrome in one UK Health Region. Joumal of Medical Genetics 38, 792-8.

Clarke D.J., Boer H., Whittington J., Holland A., Butler J. \& Webb T. (2002) Prader-Willi syndrome, compulsive and ritualistic behaviours: the first population-based survey. British Joumal of Psychiary 180, 358-62.

Butler J.V., Whittington J.E., Holland A.J., Boer H., Clarke D. \& Webb T. (2002) Prevalence of, and risk factors for, physical ill-health in people with Prader-Willi syndrome; a population-based study. Developmental Medicine E Child Neurology 44, 248-55.

Boer H., Holland A., Whittington J., Butler J., Webb T. \& Clarke D. (2002) Psychotic illness in people with Prader Willi syndrome due to chromosome 15 maternal uniparental disomy. Lancet 359, 135-6.

Whittington J.E., Holland A.J., Webb T., Butler J.V., Clarke D.J. \& Boer H. (2002) Relationship between clinical and genetic diagnosis of Prader-Willi syndrome. Joumal of Medical Generics 39, 926-32.

Webb T., Whittington J., Clarke D., Boer H., Butler J. \& Holland A. (2002) A study of the influence of different genotypes on the physical and behavioral phenotypes of children and adults ascertained clinically as having PWS. Clinical genetios $62,273-81$.

Holland A.J., Whittington J.E., Butler J., Webb T., Boer H. \& Clarke D. (2003) Behavioural phenotypes associated with specific genetic disorders: evidence from a population-based study of people with Prader-Willi syndrome. Psychological Medicine 33, 141-53.

Whittington J., Holland A., Webb T., Butler J., Clarke D. \& Boer H. (2004) Cognitive abilities and genotype in a population-based sample of people with Prader-Willi syndrome. Joumal of Intellectual Disability Research 48, 172-87.

Whitrington J., Holland A., Webb T., Butler J., Clarke D. \& Boer H. (2004) Academic underachievement by people with Prader-Willi syndrome. Joumal of Intellectual Disability Research: 48,188-200. 
Beber E. \& Boer H. (2004) Development of a specialised forensic service for women with learning disability: The first three years. British Joumal of Forensic Practice. In Press.

Stauder J.E.A., Boer H., Gerits. R.H.A., Tummers A., Whittington J. \& Curfs L.M.G. (Submitted) Differences in behavioural phenotype between parental deletion and maternal uniparental disomy in Prader-Willi syndrome.

Boer H., StauderJ.E.A., Gerits R.H.A., Tummers A., Whittington J., Webb T.\& Curfs L.M.G. (Submitted) Prader-Willi syndrome: Behavioural differences between deletion and disomy subtypes.

Collin P.J.L., Boer H., Vogels A. \& Curfs L.M.G. (Submitted) Psychose bij kinderen met Prader-Willi syndroom. 
Appendix 1

Maladaptive behaviour in Prader-Willi syndrome in adult life

Published as:

Clarke D., Boer H., Chung M.C., Stumey P. \& Webb T. (1996) Maladaptive belaviour in Prader-Willi syndrome in adult life. Joumal of Intellenal Disability Rescarth 40 , $159-65$. 


\section{Abstract}

Thirty adults with Prader-Willi syndrome (PWS) were compared with 30 adults with non-specific learning disability matched for age, sex and severity of mental retardation. Maladaptive behaviour was assessed with the Aberrant Behavior Checklist (ABC), a 58-itern structured interview which rates behaviours from 0 (not a problem) to 3 (severe problem) and which yields five factors (1) irritability, agitation; (II) lethargy, withdrawal; (III) stereotypic behavior; (IV) hyperactivity. noncompliance; and (V) inappropriate speech). The PWS sample had significantly higher factor $\mathbb{I}(P<0.001)$ and factor $V(P<0.05)$ scores. The PWS sample had mean scores above 1 for $17 \mathrm{ABC}$ items; the contrast subjects had no mean scores above 1 . The factor I scores for the PWS sample were similar to those of inpatients in hospital facilities for adults with mental retardation and mental illness or severely challenging behaviour. The results support previous work, and extend it by suggesting that temper tantrums, self-injury, impulsiveness, lability of mood, inactivity and repetitive speech are characteristic behaviours in PWS in adult life. Studies of the reasons for heterogeneity in behaviour are now needed.

\section{Introduction}

Prader-Willi syndrome (PWS) includes neonatal hypotonia and feeding difficulty, followed by hyperphagia during early childhood. Affected adults are of short stature, with hypogonadism, small hands and feet, a characteristic facial appearance and cognitive impairment. Most adults with the condition are obese, some very markedly so. About $70 \%$ of people with $\mathrm{PWS}$ have a deletion affecting the long (q) arm of chromosome 15 (q11-13), and many of the others have matemal disomy or some other abnomality which results in the absence of a patenally derived contribution to $15 q$ (Prader a al 1956, Butler 1990, Knoll er al. 1993. Nicholls 1993). Prader-Willi syndrome has an estimated incidence of one in 10000 live-born infants (Holm 1981) and a prevalence of around one in 15000 people up to 25 years of age (Akefeldt ef al. 1991).

Various maladaptive behaviours have been reported in association with PWS, including temper tantrums, self-injury through picking or scratching at skin, sleep disorders, and oppositional or stubborn behaviour, in addition to the food-seeking behaviours which occur universally in the condition (Hall \& Smith 1972, Greenswag 1987; Gupta er al. 1987; Whitman 8 Accardo 1987; Clarke et al. 1989; Borghgraef ef al. 1990; Curts et al. 1991). One study employed the Child Behavior Checklist (Achenbach \& Edelbrock 1983) and found that $87 \%$ of 27 children and adolescents with PWS had scores in the 'clinical' range, with no significant differences between 'internalizing' or 'externalizing' behaviours. The 
youngsters with IQs below 70 were not found to have significantly higher scores for maladaptive behaviours than those with IQs above 70 (Curfs et al. 1991).

\section{Need for studies with contrast groups}

Most studies of behaviour in PWS have been reports of cases or series of patients with PWS of various ages, employing clinical impressions or postal questionnaires to obtain behavioural data. Curfs (1992) observed that "the absence of systematically gathered data on behavioural characteristics, including socio-emotional functioning and personality characteristics, is striking'. This gap in knowledge is especially marked for adults with PWS. Behavioural abnomalities have been suggested as components of diagnostic criteria for PWS (Holm et al. 1992). Therefore, it is of importance to determine whether the maladaptive behaviours which have been described are truly typical of PWS, and thus, components of a behavioural phenotype, or whether they are epiphenomena associated with cognitive impairment or some other intervening or associated variable. For this reason, the present study compared PWS adults with contrast subjects matched for age, sex and severity of mental retardation. There is some evidence that maladaptive behaviour in PWS changes with age or development (Whitman \& Accardo 1987 , 1989). The study presented here tried to minimize the confounding effect of such changes by studying adult subjects. Adulthood was regarded as beginning at the age of 16 years because this convention has been used in previous studies of PWS, and allows results to be compared.

The study assessed the maladaptive behaviours of adults with PWS and those of adults with learning disability using social service and voluntary sector community residential facilities. A widely used standardized instrument was used to effect the comparison.

\section{Method}

The Prader-Willi syndrome sample

Thirty adults with PWS (i.e. aged 16 years or older) were recruited through the Prader-Willi Syndrome Association (UK) and from other sources (via colleagues practising psychiatry, psychology and social work). In view of the rarity of PWS, the biases inherent in this method of recruitment were accepted. Of the 30 people, 21 had a deletion of $15 \mathrm{q}(11-13)$ (four of these deletions were only detectable by molecular studies), two had maternal heterodisony and one had additional material on $15 q$. Five people had a firm clinical diagnosis of PWS (neonatal hypotonia and feeding difficulty, overeating in childhood, small hands and feet, characteristic 
Table 1. Summary of individual characteristics for the Prader-Will syndrome*

\begin{tabular}{|c|c|c|c|c|c|}
\hline $\begin{array}{l}\text { Age } \\
\text { (years) }\end{array}$ & $\operatorname{sex}$ & Residence & Genetics & $\begin{array}{l}\text { Height } \\
\text { (m) }\end{array}$ & $\begin{array}{l}\text { Body mass } \\
\text { index }\left(\mathrm{kg} \mathrm{m}^{-2}\right)\end{array}$ \\
\hline 16.0 & $F$ & $\mathrm{FH}$ & Del & 1.52 & 20.91 \\
\hline 16.5 & $F$ & $\mathrm{FH}$ & Delt & 1.50 & 42.84 \\
\hline 17.5 & $F$ & $\mathrm{FH}$ & Del. & 1.50 & 42.89 \\
\hline 19.0 & 䵓 & $\mathrm{FH}$ & $U / K$ & 1.57 & 37.40 \\
\hline 19.0 & $M$ & $\mathrm{FH}$ & Dell & 1.72 & 38.68 \\
\hline 19.5 & $M$ & $\mathrm{FHH}$ & $\begin{array}{l}\text { Additional } \\
\text { material } 15 q\end{array}$ & 1.55 & 38.54 \\
\hline 20.5 & $M$ & $\mathrm{FH}$ & Del & 1.57 & 28.82 \\
\hline 21.0 & $F$ & $\mathrm{FH}$ & Del & 1.42 & 45.69 \\
\hline 21.5 & $F$ & CRF & Delt+ & 1.47 & 30.74 \\
\hline 21.5 & $M$ & $\mathrm{FH}$ & Det & 1.65 & 4.7 .98 \\
\hline 22.0 & $M$ & $\mathrm{FH}$ & $U / K$ & 1.65 & 35.11 \\
\hline 22.5 & $M$ & $\mathrm{FH}$ & Del. & 1.60 & 28.01 \\
\hline 22.5 & $F$ & $\mathrm{FH}$ & Del. & 1.22 & 45.03 \\
\hline 23.5 & H & $\mathrm{FHH}$ & Dell+ & 1.50 & 35.33 \\
\hline 23.5 & $M$ & CRF & Dell & 1.60 & 24.49 \\
\hline 23.5 & $M$ & CRF & Dell & 1.58 & 26.83 \\
\hline 24.0 & $F$ & CRF & $U / K$ & 1.60 & 57.30 \\
\hline 25.0 & $M$ & $\mathrm{FH}$ & Del & 1.54 & 39.28 \\
\hline 26.0 & $F$ & CRF & Del. & 1.39 & 57.72 \\
\hline 26.5 & $F$ & $\mathrm{FH}$ & Del & 1.22 & 37.63 \\
\hline 26.5 & $M$ & CRF & NAD & 1.63 & 38.08 \\
\hline 27.5 & $M$ & CRF & Del & 1.68 & 27.73 \\
\hline 29.0 & $M$ & $\mathbb{F H}$ & Del* & 1.70 & 34.60 \\
\hline 29.5 & $F$ & CRF & $U / K$ & 1.42 & 30.15 \\
\hline 29.5 & $M$ & $\mathbb{F H}$ & Del & 1.60 & 89.49 \\
\hline 32.5 & $F$ & CRF & MHD & 1.52 & 29.44 \\
\hline 33.5 & $F$ & Hosp & Del & 1.42 & 62.43 \\
\hline 37.5 & $F$ & $\mathrm{FH}$ & Del & 1.47 & 38.19 \\
\hline 37.5 & $F$ & CRF & MHD & 1.42 & 27.23 \\
\hline 44.0 & $F$ & CRF & $U / K$ & 1.42 & 53.32 \\
\hline
\end{tabular}

*Del, deletion of 15q(11-13); MHD, maternal heterodisomy; U/K, unknown; FH, family home; NAD, no abnormality detected; CRF, community residential facility: Hosp, hospital.

+Deletion detectable only through DNA studies.

Table 2. Maladaptive behaviour: mean ABC factor scores for Prader-Willi syndrome subjects and contrast subjects resident in community residential facilities

\begin{tabular}{lllllll} 
& Factor & & & & & \\
& I & II & III & IV & V & Total \\
Prader-Willi syndrome & 18.8 & 6.8 & 0.96 & 9.2 & 3.93 & 39.9 \\
Community residential facility & 6.2 & 6.6 & 2.4 & 9.2 & 2.2 & 26.6 \\
P & $<0.001$ & NS & NS & NS & $<0.05$ & NS \\
\hline
\end{tabular}


Table 3. Maladaptive behaviour: ABC items and mean scores for 30 Prader-Will syndrome (PWS) subjects and 30 matched community residential facility (CRF) subjects.

\begin{tabular}{lllll}
\hline & PWS & CRF & & \\
\hline 1 & Excessively active & 0.07 & 0.7 & 0.0074 \\
2 & Injures self & 1.43 & 0.2 & $<0.0001$ \\
3 & Listless, sluggish, inactive & 1.3 & 0.56 & 0.0012 \\
10 & Temper tantrums & 2.13 & 0.9 & 0.0001 \\
22 & Repetitive speech & 1.2 & 0.53 & 0.0081 \\
25 & Depressed mood & 0.87 & 0.3 & 0.0097 \\
28 & Does not pay attention to instructions & 1.27 & 0.5 & 0.0044 \\
29 & Demands must be met immediately & 1.63 & 0.77 & 0.0022 \\
34 & Cries over minor hurts and annoyances & 1.23 & 0.2 & 0.0001 \\
46 & Repeats a word or phrase over and over & 1.17 & 0.37 & 0.0018 \\
47 & Stamps feet while banging objects or slamming doors & 1.2 & 0.3 & 0.002 \\
50 & Deliberately hurts him/herself & 1.3 & 0.13 & $<0.0001$ \\
51 & Pays no attention when spaken to & 0.87 & 0.27 & 0.0049 \\
52 & Does physical violence to self & 1.17 & 0.1 & 0.0002 \\
57 & Throws, temper tantrums when he/she does not get own way & 2.13 & 0.67 & $<0.0001$ \\
\hline
\end{tabular}

Table 4. Comparison of ABC factor scores of the Prader-Willi syndrome group by body mass index

\begin{tabular}{lllllll}
\hline & Factor & & & & & \\
& I & 11 & III & IV & V & Total \\
\hline Ten subjects with highest body mass indices & 25.3 & 630 & 0.7 & 11 & 3.6 & 46.9 \\
Ten subjects with lowest body mass indices & 13 & 6.1 & 1 & 5.3 & 3.8 & 29.2 \\
$P$ & 0.023 & NS & NS & 0.03 & NS & 0.041 \\
\hline
\end{tabular}

dysmorphology, short stature and hypogonadism as adults, and an independent diagnosis of PW/S by a consultant paediatrician), but did not have chromosome studies performed or had studies performed which were monchuswe (a. because a parent had died and matemal disomy could not be excluded or confirmed), One man had a firm clinical diagnosis and apparenty nommal chromosome 15s, although the presence of a very small deletion could not be excluded. Diagnostic criteria such as those of Holm at (1992) were rot used because the latter include behavioural components. Detals of the sample are given in Table 1. Two women received progestogen treatment for possble premenstrual symptoms, one woman received diuretics and bronchodilating agents because of heart failure and respiratory problems related to obesty, and one man received medication for heart falure and topical treament for ulcerared pick lesions on his legs. One woman took insulin for diabetes melfitus, anotizer received chlorpropamide and a further female subject received netormin. One man received intra-muscular testosterone, another took clompramine, haloperidol, frusemide and ciprexin, and one man received lactulose daily. One 
woman took chlorpromazine and amitriptyline, another had been diagnosed as having a psychotic illness and received fluphenazine decanoate injections, and a third was diagnosed as having a delusional disorder and treated with tupenthixol. No subject was actively mentally ill at the time of the assessment. About one-third of the sample had probllems with excessive sleepiness during the day, usually when travelling by car or bus or in understimulating environments. Other subjects had a history of such sleepiness, but their carers felt that this did not constitute a problem at the time of assessment. No subject was described as having cataplexy.

\section{Community residential facilities (CRF) contrast group}

This consisted of 30 people matched for age ( \pm 18 months), sex and degree of mental retardation (using ICD-10 Clinical Descriptions and Diagnostic Guidelines; WHO 1992) who were resident in, or received respite care in, social service or voluntary sector facilities for people with learning disabilities, and who did not have an identified syndronal cause for their learning problems. Subjects were not recruited from community residential facilities (CRFs) providing residential or respite care for people with severe sensory impaiments, or autistic disorders. Maladaptive behaviour was assessed with the Aberrant Behavior Checklist (ABC) (Aman ot al. 1985a, b, 1987), a 58-item structured interview which requires a rating of specified maladaptive behaviours from 0 (not at all a problem) to 3 (the problem is severe in degree). The ABC yields five factors or subscales: (I) irritability, agitation; (II) lethargy, withdrawal; (III) stereotypic behaviour; (IV) hyperactivity, noncompliance; and (V) inappropriate speech. Factor and item scores were used to compare the three groups. The $\mathrm{ABC}$ was used because its reliability and validity in assessing maladaptive behaviour among people with cognitive impaiment has been demonstrated (Aman et al. 1987; Newton \& Stumey 1987), it includes behaviours such as loss of temper and non-compliance which previous studies have suggested are frequent among adults with PWS, it has been recommended for use in studies of belhaviour in developmental/dysmorphology syndromes (O'Brien 1991), and it was found to be the most appropriate measure of its type in a pilot study.

Four of the PWS group had experienced psychotic symptoms during adult life (three are described in Clarke 1993), but these symptoms were not prominent for any of the subjects at the time of assessment with the $\mathrm{ABC}$.

\section{Results}

Results are summarized in Tables $1-4$. The PWS sample had significanty higher scores than the CRF sample for $A B C$ factor $I(P<0.001)$ and factor $V$. The statis- 
tical calculations of significance in Tables 2,3 and 4 were calculated using the Mann-Whitney non-parametric test, employing Minitab 9 software to compare data sets. The PWS sample had mean scores above 1.0 for 17 items, whereas the CRF group had no $A B C$ items with a mean score exceeding 1 . The PWS group had higher scores for items relating to tempers, impulsiveness and non-compliance, mood disorder, inactivity, and repetitive speech, supporting previous reports of maladaptive behaviours associated with the disorder. Although the higher scores of the PWS group imply a greater problem as perceived by carers, the magnitude of this difference cannot be assessed ( $A B C$ ratings are ordinal in nature). Younger PWS subjects had marginally higher scores than older subjects. Factor scores did not differ significantly between men and women with PWS. No linear relationship was found between age or body mass index and factor scores, with the possible exception of factor I scores and body mass index (correlation coefficient. 0.361 ). The most overweight PWS subjects had significantly higher factor I and factor IV scores than those least overweight-for-height.

\section{Discussion}

The high scores of the PWS sample for factor I of the ABC supports previous research implying that tempers, self-injury, impulsiveness, lability of mood, inactivity and repetitive speech (such as persistent questioning) are particularly problematic behaviours for people with PWS. No relationship was found between sex and factor scores. No linear relationship was found between age, or body mass index and $\mathrm{ABC}$ factor scores (with the possible exception of a modest correlation between body mass and factor I score), but further research with larger numbers is necessary before definite conclusions can be drawn. The results are compatible with previous work suggesting that maladaptive behaviours in PWS may change with development or ageing. The trend to higher factor I scores among the youngest subjects in the study may reflect the increase in maladaptive behaviour described by some authors during adolescence and early adult life in PWS (e.B. Whitman \& Accardo 1987, 1989).

Standardized instruments such as the $\mathrm{ABC}$ are invaluable for enabling comparisons between groups across a range of conmonly occurring maladaptive behaviours, but comparisons of factor scores may mask differences which relate to specific items within factors. This is especially relevant when factors combine items relating to disparate constructs such as lethargy and social withdrawal, for example, lethargy appears to be a problem for many people with PWS, but social withdrawal does not.

Greenswag (1987) found the following behaviours to be relatively frequent and prominent among 232 adults with PWS in the USA: Slow moving, good natured; 
stubborn, sleepy, belligerent (food-related), irntable; impulsive; lazy; belligerent (non-food related); antisocial with peers; moody; antisocial with family; physically aggresswe". These items overlap to a considerable extent with those in Table 3 , implying that some progress is being made in delineating the behavioural phenotype of PWS. Further studies with comparison groups will facilitate this process.

The selection of contrast groups for studies of maladaptive behaviour among people with syndromal causes of leaming disability is difficult. The comparison with the CRF group was intended to provide a contrast which would be clinically meaningful and allow limited quantification of the PWS sample's maladaptive behaviours (i.e. Whether a mean item or factor score exceeded, was the same as or was less than that of a contrast group). An attempt was made to find age-, sex-and severity-of-retardation-matched contrast subjects who were patients in hospital facilities for people with leaming disability and mental illness or severely challenging behaviour. Unfortunately, matching was not possible for degree of mental retardation, and only older PWS subjects could be matched for age (few people below the age of 20 are admitted to such facilities in the West Midlands). The mean factor $I$ score of 22 such inpatients matched for age and sex to the older PWS subjects was 12.96 , compared to 17.68 for the PWS subjects to whom they were matched. This implies that the perceived problem posed by some of the maladaptive behaviours which are most troublesome for people with PWS is comparable to that posed by in-patients in specialist challenging behaviour services.

Prader-Willi syndrome is associated with many secondary features (e.g. obesity and sleep disorders) which may influence the nature or extent of comexistent maladaptive behaviours, but which cannot be easily controlled for by using. contrast groups. Sleep disorders are well-documented in association with PWS (Helbing-Zwanenburg et al. 1993) and many carers believe that they contribute to daytime irritability and loss of temper. Thus, sleep disorders may contribute to the high factor $\llbracket$ scores which seem characteristic of PWS. Other aspects of PWS which may modify the type or extent of maladaptive behaviour associated with the disorder include the age at which dietary restrictions are imposed and their effectiveness, the nature and availabilicy of support services, and the effect of obesity on self-image and peer relations.

The group of people with PWS studied may have been biased towards those with more severe behavioural problems, but in view of the rarity of PWS among the adult population, and the lack of previous research employing standardized instruments and contrast groups, the methodological weaknesses associated with studying a suiall and possibly biased sample were accepted. A further methodolog ical weakness is inherent in any sudy which employs instruments requiring a judgement by carers about the severity of "problem" posed by behaviours. Individual tolerance of behaviour varies. However, the instrument used has high 
test-retest and inter-rater reliability and concurtent validicy with other instruments and with ratings derived from direct observation. (Newton \& Stumey 1988). Other strategies for assessing maladaptive behaviour (such as direct observation) are also associated with methodological problems (such as changes consequent on the presence of an observer).

This study represents the first attempt to extend into adult life the use of standardized measures and comparison groups to describe the malaptive behaviours which are so characteristic of PWS as to constitute a behavioural phenotype. Behavioural problems are commonly cited by parents and other carers as among the most difficult challenges posed by PWS. Studies with an emphasis on the heterogeneity of behaviour, and the reasons for this, may lead to the development of effective interventions.

\section{Acknowledgements}

We are grateful to the people with PWS and their carers who took part in the research programme, to the nursing and social service staff who gave their time to complete the $\mathrm{ABC}$ for the contrast groups, and to colleagues for their helpful comments on the manuscript.

\section{References}

Achenbach T.M. \& Edelbrock C.S. (1983) Mamal for the Child Befowiop Chedklist and Revised Child Profile. University of Vermont, Burlington, VT.

Akefeldt A., Gilliberg C. \& Larsson C. (1991) Prader-Willi syndrome in a Swedish rural connty: epidemiological aspects. Developmental Medicine and Child Neurology 33, 715-21.

Aman M.G., Richmond G., Stewart A.W., Bell J.C. \& Kistell R.C. (1987) The Abermant Behavior Checklist: factor structure and effect of subject variables in Anerican and New Zealand facilities. American Jownal of Mental Deficiono 91, 570-8.

Aman M.G., Singh N.N., Stewart A.W. \& Field C.J. (1985a) The Aberant Behavior Checklist: a behavior rating scale for the assessment of treatment effects. Amerian Joumal of Mettal Deficiency $89,485-91$.

Aman M.G., Singh N.N., Stewart A.W. \& Field C.J. (1985b) Psychometric chatacteristics of the Aberrant Behavior Checklist. American Jound of Mental Deficiency 89, 492-502.

Borghgraef M. Fryns J.P. \& Van den Berghe H. (1990) Psychological profile and behavioral characteristics in 12 patients with Pradier-Willisyndrome. Genetrc Connselling $38,141-50$.

Butler M.G. (1990) Prader-Willi syndrome: current understanding of cause and diagnosis. American Joumal of Medical Genetios 35, 319-32.

Clarke D.J. (1993) Prader-Will syndrone and psychoses. Brifish Journal of Psyohatry 163, 680-4. 
Clarke D.J. Waters] \& CorbettI.A. (1989) Adults with Prader-Willi syndrome: abnormalities of sleep and behaviour. Joumal of the Ropal Society of Medicine 82,21-4.

Curs L.M.G. (1992) Psychological profile and behawioral characteristics in the Prader-Will syndrone. In: Prader Willi Syndwome and Other Chromosome 15 q Deletion Disorders. NATO ASI Series H: Cell Biology, Vol. 61 (ed. S. B. Cassidy), pp. 211-21. Springer-Verlag, Berlin.

Curfs L.M.G., Verhullst F.C. \& Fryns J.P. (1991) Behavioural and emotional problems in youngsters with Prader-Willi syndrome. Cenetic Counelling 2, 33-41.

Greenswag L.R. (1987) Adults with Prader-Willi syndrome: a survey of 232 cases. Developmental Medicine and Child Neuplogy 29,145-52.

Gupta B.K., Fish D.N. \& Yerevanian B. I. (1987) Carbamazepine for intermittent explosive disorder in a Prader-Willi syndrome patient. Jow of Clinial Pshiary $48,423$.

Hall B.D. \& Smith D.W. (1972) Prader-Willi syndrome. A resume of 32 cases including an instance of affected first cousins, one of whom is of nomal stature and inteligence. Jownt of Podiatrics $81,286-93$

Helbing-Zwanenburg B., Kamphuisen H.A.C. \& Mouttazaev M.S. (1993) The origin of excessive daytime sleepiness in the Prader-W Willi syndrome. Joumal of Huellectual Disability Rescard 37, 533-41.

Holm V.A. (1981) The diagnosis of Prader-Will syndrome. In: Prader Will Syndrone (ed. by V.A. Holn, S.J. Sulabacher \& P.I. Pipes), pp. 27-44. University Park Press, Baltimore, MD.

Holm V.A., Cassidy S.B., Butler M.G., Hanchett J.M., Greenberg F., Whitman B.Y. \& Greenswag L.R. (1992) Diagnostic criteria for Prader-Willi syndrome. In: Prader-Willi Syndrome and Other Chromosame 15q Delerion Disorders. NATO ASI Sesies H: Cell Biology, Vol. 6 (ed. by S.B. Cassidy), pp. 105-17. Springer-Verlag, Berlin.

Knoll J.H.M., Wagstaff]. \& Lahande M. (1993) Cytogenetic and molecular studies in the Prader-Willi and Angelman syndromes: an overview. American Joumal of Medical Genetics 46, 2-6.

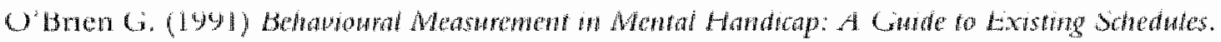
Society for the Study of Behavioural Phenotypes, Oxford.

Newton]. T. \& Stumey P. (1988) The Aberrant Bellaviour Checklist: B British replication and extension of its psychometric properties. Joumal of Mental Deficiency Resenth 32, 87-92,

Nicholls R.O. (1993) Genonic imprinting and uniparental disomy in Angellman and Prader-Willi syndromes: a review. Amerion Jomat of Medical Getetics 46, 16-25.

Prader A., Labhart A. \& Willi H. (1956) Ein Syndrom von Adipositas, Kleinwuchs, Kryptorchismus und Oligophrenie nach myatonieartigen Zustand im Neugeborenalter.

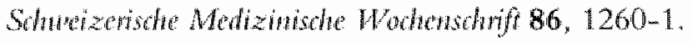

Whitman B.Y. \& Accardo P. (1987) Emotional symptoms in Prader-Willi syndrome adolescents. Ancrian Joumal of Medical Genctics 28,897-905.

Whitman B.Y. \& Accardo P. . (1989) Prader-Willi syndrome. Jownd of the Royal Sociery of Mediane 82, 448.

World Health Organization (1992) The ICD 10 Classifnation of Mental and Behaniomal Disorders: Chincal Descriptions and Diaghostic Gathelines. WHO, Geneva. 
Appendix 2

\section{Development and behaviour in two genetic syndromes:}

\section{2: Rubinstein-Taybi syndrome}

Published as:

Boer H., Langton J. \& Clarke D. (1999) Development and Behaviour in two genetic syndromes: 2: Rubinstein-Taybi Syndrome. Joumal of Applied Researd in Intellectual Disabilities $12,302-7$. 


\section{Abstract}

Development and behaviour was investigated in a sample of 44 children and adults with Rubinstein-Taybi syndrome (RTS) using the Society for the Study of Behavioural Phenotypes Postal Questionnaire. There were few differences between males and females. Children and adults were compared. Most of the RTS adults communicated using speech but expressive speech was limited. Repetitive speech and repetitive movements, werbal abuse and over-friendliness with strangers were reported. Mood abnomalities and problems with self-injury were reported for about half the people surveyed. Toilet training appeared to take a long time, but the majority of adults surveyed were fully continent.

\section{Introduction}

Rubinstein-Taybisyndrome (RTS) was first described in 1963 in a report of seven unrelated children with intellectual disabilities, similar facial appearances, and broad thumbs and broad big toes (Rubinstein \& Taybi, 1963). RTS is thought to affect around 1 in 125,000 liwe borm infants, males and females being affected equally (Hennekam ef al. 1992). The disorder was found to be associated with microdeletions at 16p13.3 (Breuning ef al. 1993). Affected people in their seventh decade have been reported (Rubinstein, 1990). Little has been written about behavioural aspects of the syndrome. Performance IQ usually exceeds verbal IQ, and one report found a mean IQ of 51 with a range from 30 to 79 (Stevens \& Caery, 1990). Some reports suggest that children with $\mathbb{R} T S$ are often happy, sociable, and sometimes over-friendly. Sleep disturbances have been reported "as have self-stimulatory activities such as rocking, spinning and hand tapping. Self-injury and outbursts of temper have also been described (Hennekam $e f$ l. 1992; Stevens \& Carey, 1990; Baxter \& Beer, 1992; Udwin \& Dennis, 1995). Levitas \& Reid (1998) described psychiatric evaluations of a clinical series of 13 patients with RTS, and reported that 8 people had mood disorders, 4 people had tics or obsessive-compulsive symptomatology and 1 person had an autistic spectrum disorder. Levitas ar Reid noted that 6 of their sample of 13 patients had neuroleptic-induced movement disorders and 3 had had at least one episode of neuroleptic malignant syndrome. Although Levitas \& Reid's sample was highly selected (having been referred "primarily because of behavioural problems") the clustering of diagnoses and the apparently high rate of adverse effects of antipsychotic nedication are noteworthy.

The recognition of such behavioural traits associated with genetic disorders is of interest and importance for the reasons described in the introduction to the 
preceding paper (Boer \& Clarke, 1999), which describes developmental and behavioural aspects of Prader-Willi syndrome.

\section{Method}

Members of the Rubinstein-Taybi Support Group were asked to complete the Society for the Study of Behavioural Phenotypes Postal Questionnaire (SSBP-PQ). Details of the SSBP-PQ and the methodology used are given in paper (1). Most of the members of the RTS Support Group are parents of children and adults with the syndrome.

\section{Results}

Ninety-five questionnaires were mailed to the Rubinstein-'Taybi Support Group, and 46 replies received, a response rate of $48 \%$. Two replies concerning children below 3 years of age have been excluded from the analysis (because the SSBP-PQ was not intended for use with children below 3 years of age). The 44 questionnaires reported related to 27 males (mean age 12 years, range $3-35$ years) and 17 females (mean age 13.5 years, range $3-40$ years). Table 1 summarises items relating to development, comparing people between the ages of 3 and 16 years with those aged 17 years and over. Table 2 summarises items relating to behaviour.

Two children with RTS wore hearing aids. Most of the adults could walk, but less steadily than other people of the same age, and most needed help to dress.

Problem behaviours included repetitive speech and verbal abuse, repetitive movements, over-friendliness with strangers, and unusual routines. Nearly half the sample was described as being abnomally happy, and many people had a very changeable mood state. About half the people surveyed had problems with self-injurious behaviour. There were few differences between males and females, but as with PWS, "Problems shopping", "Creates chaos aimlessly" "Deliberately destroys things" and some of the items relating to mood abnormality secm more problematic for males than females.

Of 13 adults with RTS, 4 conversed with no problems, 3 used long sentences, 2 used sentences with two words and links, 3 used two word sentences, and 1 indicated objects using single words. Statistical comparisons could not be made between the younger and older groups for most items, because of the small numbers of people aged over 17 years. However, there was a statistically significant difference between the proportion of younger and older people described as fully toilet trained, implying that toilet training may be delayed in R TS but that nearly all adults eventually achieve full continerace. 
Table 1. RTS: Comparison of chitdren (3-16 years) and adults (17-51 years): development.

\begin{tabular}{|c|c|c|c|c|c|}
\hline Item & $3-16$ years & $17-5.1$ years & Total & $x^{2}$ & $p$ \\
\hline \multicolumn{6}{|l|}{ Epilepsy } \\
\hline Seizure(s) in past year & $2 / 31$ & $0 / 13$ & $2 / 44$ & $\mathrm{NA}$ & - \\
\hline Medication for epilepsy & $0 / 31$ & $0 / 13$ & $0 / 44$ & NA & - \\
\hline Selzure(s) before past year & $4 / 31$ & $3 / 13$ & $7 / 44$ & $\mathrm{NA}$ & - \\
\hline \multicolumn{6}{|l|}{ Vision and hearing } \\
\hline Wears glasses & $13 / 31$ & $5 / 13$ & $18 / 44$ & 0.046 & $\mathbb{N S}$ \\
\hline Wears hearing aid & $2 / 31$ & $0 / 13$ & $2 / 44$ & NA & - \\
\hline \multicolumn{6}{|l|}{ Mobilitity } \\
\hline Can sit without support & $31 / 31$ & $13 / 13$ & $4.4 / 44$ & NA & - \\
\hline Walks steadily and normally & $8 / 31$ & $5 / 12$ & $9 / 43$ & NA & - \\
\hline Walks, not as steadily as others & $14 / 31$ & $8 / 12$ & $22 / 43$ & 1.601 & NS \\
\hline Walks, actiwity limited & $5 / 31$ & $1 / 12$ & $6 / 43$ & NA & - \\
\hline No independent walking & $4 / 31$ & $2 / 12$ & $6 / 43$ & NA & - \\
\hline \multicolumn{6}{|l|}{ Dexterity } \\
\hline Gross hand movements & $30 / 31$ & $13 / 13$ & $43 / 44$ & NA & - \\
\hline Fine hand movements & $19 / 31$ & $11 / 13$ & $30 / 44$ & NA & - \\
\hline \multicolumn{6}{|l|}{ Feeding } \\
\hline \multicolumn{6}{|l|}{ Feeds him/herself: } \\
\hline ightwot at all & $2 / 31$ & $0 / 13$ & $2 / 44$ & $\mathbb{N A}$ & - \\
\hline With fingers & $4 / 31$ & $0 / 13$ & $4 / 44$ & $\mathbb{N A}$ & - \\
\hline With spoon & $13 / 31$ & $3 / 13$ & $16 / 44$ & NA & - \\
\hline With knife and fork & $12 / 31$ & $10 / 13$ & $22 / 44$ & 5.35 & $>0.05$ \\
\hline Drinks from ordinary cup & $25 / 31$ & $13 / 13$ & $38 / 44$ & NA & - \\
\hline \multicolumn{6}{|l|}{ Continence and hygiene } \\
\hline Tollet trained & $7 / 30$ & $11 / 13$ & $18 / 43$ & 13.995 & $<0.001$ \\
\hline Washes normally & $14 / 30$ & $7 / 13$ & $21 / 43$ & 0.187 & NS \\
\hline
\end{tabular}

\section{Dressing}

Can dress self:

Completely

With little help

With a lot of help

Not at all

$\begin{array}{lllll}1 / 31 & 3 / 13 & 4 / 44 & \text { NA } & \text { - } \\ 13 / 31 & 8 / 13 & 21 / 44 & 1.411 & \text { NS } \\ 6 / 31 & 2 / 13 & 8 / 44 & \text { NA } & \text { - } \\ 11 / 31 & 0 / 13 & 11 / 44 & \text { NA } & \text { - }\end{array}$

\section{Communication}

Does not indicate needs

Uses nonwerbal method

Uses formal signs or symbols

$0 / 30$

0,13

$0 / 43$

$6 / 30$

$0 / 13$

$6 / 43$

$3 / 30$

$1 / 13$

21/30

$12 / 13$

$4 / 43$

$33 / 43$

$\begin{array}{ll}\text { NA } & - \\ \text { NA } & - \\ \text { NA } & - \\ \text { NA } & -\end{array}$


Table 2. RTS: Comparison of children (3-16 years) and aduits (17-51 years): behaviour.

\begin{tabular}{|c|c|c|c|c|c|}
\hline Item & $\begin{array}{l}3-16 \\
\text { years }\end{array}$ & $\begin{array}{l}17-51 \\
\text { years }\end{array}$ & Total & $x^{2}$ & $p$ \\
\hline \multicolumn{6}{|l|}{ Feeding } \\
\hline Eats normal amount of food & $22 / 31$ & $8 / 13$ & $30 / 44$ & NA & . \\
\hline Eats less than normal & $6 / 31$ & $3 / 13$ & $9 / 4.4$ & NA & - \\
\hline Eats more than normal & $3 / 31$ & $2 / 13$ & $5 / 4 \cdot 4$ & $N A$ & - \\
\hline Pica & $8 / 31$ & $2 / 13$ & $10 / 44$ & NA & - \\
\hline Faddy eating & $9 / 30$ & $3 / 13$ & $12 / 43$ & NA & \\
\hline \multicolumn{6}{|l|}{ Sleep } \\
\hline Regular pattern of sleep & $25 / 31$ & $12 / 13$ & $37 / 44$ & NA & - \\
\hline Repeated waking & $11 / 29$ & $5 / 13$ & $16 / 42$ & NA & - \\
\hline Excessive daytime slleepiness & $2 / 31$ & $0 / 13$ & $2 / 44$ & MA & 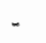 \\
\hline \multicolumn{6}{|l|}{ Social behaviour } \\
\hline Isolated/in world of own & $7 / 31$ & $7 / 13$ & $14 / 44$ & NA & - \\
\hline Too friendly with strangers & $22 / 30$ & $4 / 12$ & $26 / 42$ & NA & - \\
\hline Unusual eye contact & $8 / 29$ & $4 / 12$ & $12 / 41$ & NA & - \\
\hline Abnormal facial expression/gesture & $11 / 30$ & $4 / 13$ & $15 / 43$ & NA & - \\
\hline Problems shopping & $8 / 31$ & $6 / 13$ & $14 / 44$ & NA & - \\
\hline \multicolumn{6}{|l|}{ Activity } \\
\hline Underactive & $7 / 31$ & $2 / 13$ & $9 / 44$ & NA & - \\
\hline Normally active & $18 / 31$ & $8 / 13$ & $26 / 44$ & 0.046 & NS \\
\hline Overactive & $6 / 31$ & $3 / 13$ & $9 / 4.4$ & MA & - \\
\hline Clumsy & $26 / 31$ & $9 / 12$ & $35 / 43$ & NA & - \\
\hline Creates chaos aimlessly & $13 / 30$ & $3 / 13$ & $16 / 43$ & NA & - \\
\hline \multicolumn{6}{|l|}{ Communication } \\
\hline Echolalia & $16 / 28$ & $11 / 13$ & $27 / 41$ & NA & - \\
\hline Shouts or screams unexpectedly & $12 / 31$ & $3 / 12$ & $15 / 43$ & NA & - \\
\hline \multicolumn{6}{|l|}{ Unusual movements and interests } \\
\hline Repetitive movements & $24 / 31$ & $5 / 13$ & $29 / 44$ & NA & - \\
\hline Attachment to peculiar object(s) & $10 / 31$ & $6 / 13$ & $16 / 44$ & NA & - \\
\hline Unusual routines & $16 / 30$ & $9 / 13$ & $25 / 43$ & 0.942 & NS \\
\hline \multicolumn{6}{|l|}{ Aggression and self-injury } \\
\hline \multicolumn{6}{|l|}{ Aggressive towards: } \\
\hline Children & $6 / 31$ & $1 / 13$ & $7 / 44$ & NA & . \\
\hline Adults & $4 / 31$ & $2 / 13$ & $6 / 44$ & NA & - \\
\hline Self-injurious behaviour & $14 / 31$ & $7 / 13$ & $21 / 44$ & 0.277 & $\mathbb{N S}$ \\
\hline Verbally abusive & $25 / 20$ & $11 / 13$ & $36 / 42$ & NA & - \\
\hline Stubborn & $6 / 24$ & $5 / 11$ & $11 / 35$ & NA & * \\
\hline Deliberately destroys things & $7 / 31$ & $2 / 13$ & $9 / 44$ & MA & - \\
\hline \multicolumn{6}{|l|}{ Anxiety and mood abnormality } \\
\hline Excessively happy & $14 / 31$ & $5 / 13$ & $21 / 44$ & 0.168 & NS \\
\hline Excessively unhappy & $5 / 29$ & $2 / 13$ & $7 / 42$ & MA & - \\
\hline often fiearful & $6 / 29$ & $3 / 13$ & $9 / 42$ & NA & - \\
\hline Mood very changeable & $10 / 30$ & $2 / 13$ & $12 / 43$ & NA & . \\
\hline Senous temper tantrums (at least weekly) & $8 / 31$ & $5 / 13$ & $13 / 44$ & NA & - \\
\hline
\end{tabular}




\begin{tabular}{|c|c|c|c|c|c|}
\hline Item & $\begin{array}{l}\text { 3-16 } \\
\text { years }\end{array}$ & $\begin{array}{l}17-51 \\
\text { years }\end{array}$ & Total & $x^{2}$ & $p$ \\
\hline \multicolumn{6}{|l|}{ Anxiety and mood abnormality } \\
\hline Excessively thappy & $14 / 31$ & $5 / 13$ & $21 / 44$ & 0.168 & NS \\
\hline Excessively unhappy & $5 / 29$ & $2 / 13$ & $\pi / 42$ & NA & - \\
\hline Often fearful & $6 / 29$ & $3 / 13$ & $9 / 42$ & NA & - \\
\hline Mood wery changeable & $10 / 30$ & $2 / 13$ & $12 / 43$ & NA & - \\
\hline Serious temiper tantrums (at least weekly) & $8 / 31$ & $5 / 13$ & $13 / 44$ & NA & - \\
\hline
\end{tabular}

\section{Discussion}

Little previous work has been published regarding the behaviour of people with RTS. Although 44 children and adults with RTS constitutes a relatively small sample, the findings are consistent with reports suggesting that over-friendliness, abnormal mood states (especially elevated mood), and repetitive speech and acts may be problematic for people with RTS. Levitas \& Reid (1998) reported a prevalence of mood disorder of $61 \%$ in their (highly selected) clinic sample of 13 people with RTS referred for evaluation of behavioural abnormalities. Three people in their sample had a diagnosis of obsessive compulsive disorder, one person "possible" obsessive compulsive disorder, and one person "obsessive compulsive features."

Verbal abuse was reported relatively frequently in the present study. Self-injury and temper "tantrums" were problems for a substantial minority. The overall pattern of development and of emotional and behavioural problems associated with RTS differs from that found in Cri du chat syndrome by Cornish \& Pigram (1996) and from that reported by Boer \& Clarke (1999), although RTS and PWS seem to share a vulnerability to mood disorder on the basis of the present reports and those of Levitas \& Reid (1998), Beardesmore et al. (1998), Clarke et al. (1998), Clarke (1998), and Verhoeven ef al. (1998).

Problems with adaptive behaviours such as mobility and self-care skills were more pronounced among the people with R TS reported here than among the sample of people with Prader-Willi syndrome reported in paper (1), reflecting the more severe cognitive and physical disability usually associated with RTS.

The differences between males and females in the RTS sample may be a reflection of the differences observed in the prevalence of conduct and emotional disorders in children of different genders, rather than an effect specific to RTS (most of the sample were aged below 16 years).

The methodological problems and other limitations of the research reported here are summarised in paper (1) (Boer \& Clarke, 1999), including the focus on prob- 
lematic behaviours. The authors are aware that many carers report positive temperamental characteristics in association with RTS, and it is also important to stress that there is considerable variation in the prevalence of problem behaviours between individuals and over time. Research of this nature is useful to describe constellations of behaviours and emotional states that may be relatively characteristic of a genetic disorder. However, not all people with a particular disorder will have the problems commonly described; the problems faced by an individual with a genetic syndrome will also be influenced by other genetic contributions and the effects of the environment, and of development contributing to the enviromment, in a complex interactive fashion. The purpose of describing possible vulnerabilities to particular behaviours and emotional states is as much to allow exploration of environmental factors that influence the problems fand that could, therefore, be used in interventions) as to correlate such disorders with genetic abnormalities. Further research which includes positive behavioural characteristics, and a longitudinal perspective, would allow professionals to present a more accurate and rounded picture of the behavioural profile associated with R.TS and longitudinal studies would help to clarify changes in behaviour over time.

\section{Acknowledgements}

The authors are grateful to the Rubinstein-Taybi Support Group for their invaluable support and practical help, to the carers who completed the SSBP-PQ, and to the people with RTS who agreed to take part in the study. We are also grateful to the University of Birmingham Department of Psychiatry and South Birmingham Mental Health Trust for access to data-analysis, photocopying and other facilities, and to Dr Greg O'Brien for the SSBP-PQ reliability data.

\section{References}

Baxter G. \& Beer J. (1992) Rubinstein-Taybi syndronne. Psplologial Repors 70 451-6. Beardsmore, A., Doman T., Cooper S.-A. \& Webb T. (1998) Afective psychosis and

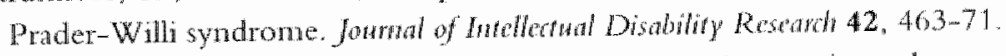

Boer H. \& Clarke D. (1999) Development and behavour in genetic syndromes: l"rader-Willi Syndrome. Joumal of Applied Researh in Intelletual Disabilitios 12, 294-301.

Breuning M.H. Dauwerse H.G., Fugazza G. Saris J. Sprut L., Winen H., Tommerup. N. van der Hagen C.B., Inaizumi K. et al. (1993) Rubinstein-Taybi syndrome cansed by

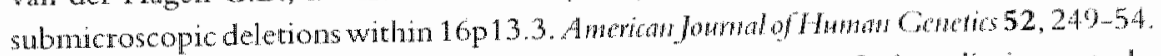

Clarke 1. (1998) Prader-Will syndrome and psychotic symptonn: 2. A preliminary study of prevalence using the Psychopathology Assesment for Adults with Developincntal Disability checklist. Jonmal of Intelleatial Disability Rosath 42,445-54. 
Clarke D.J. Boer H., Webb T., Scort P., Frazer S., Vogels A., Borghgraef M. \& Curts L.M.G. (1998) Prader-Willi syndrome and psychotic symptomas: 1. Case descriptions and genetic studies. Jourfal of Ihtellecual Disability Research 42, 440-50.

Comish K.M. \& Pigrand J. (1996) Developmental and behavioural characteristics of cri du chat syndrome. Arehives of Disease in Childhood 75, 448-50.

Hennekan R.C.M. Baselier A.C.A., Beyaert E., Bos A., Blok J.B., Jansma H.B.M., Thorbecke-Nilsen V.V. \& Veerman H. (1992) Psychological and speech studies in Rubinstein-Taybi syndrome. Anverican Jowntal on Mental Retardation 96, 645-60.

Levitas A.S. \& Reid C.S. (1998) Rubinstein-Taybisyndrome and psychiatric disorders. Joumal of Wredlectual Disabilify Research $42,284-92$.

Rubinstein J.H. (1990) Broad thumb-hallux (R ubinstein-Taybi) syndrome 1957-1988. Anew ican Joumal of Medrion Genetic Suppl. 6, 3-16.

Rubirstein J.H. \& Taybi H. (1963) Broad thumbs and toes and facial abnormalities. A possible mental retardation syndrome. American Jownal of Diseases of Children 105, 588-608.

Stevens C.A., Carey J.C. \& Blackburn B.L. (1990) Rubinstein-Taybi syndrome: a natumal history study. American Jow of Medical Genetics Suppl. 6, 30-7.

Udwin O \& Dennis J. (1995) Psychological and behavioural phenotypes in genetically determined syndromes: a review of research findings. In: Behavionral Phenotypes (eds $\mathrm{G}$. O'Brien \& W. Yule), pp. 165-7. Mackeith Press, London.

Verhoeven W.M.A., Curfs L.M.G. \& Tuinier S. (1998) Prader-Willi syndrome and cycloid psychoses Joumal of Intellectual Disability Researd 42, 455-62. 